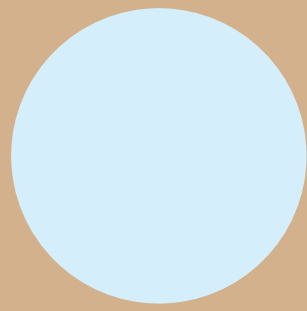

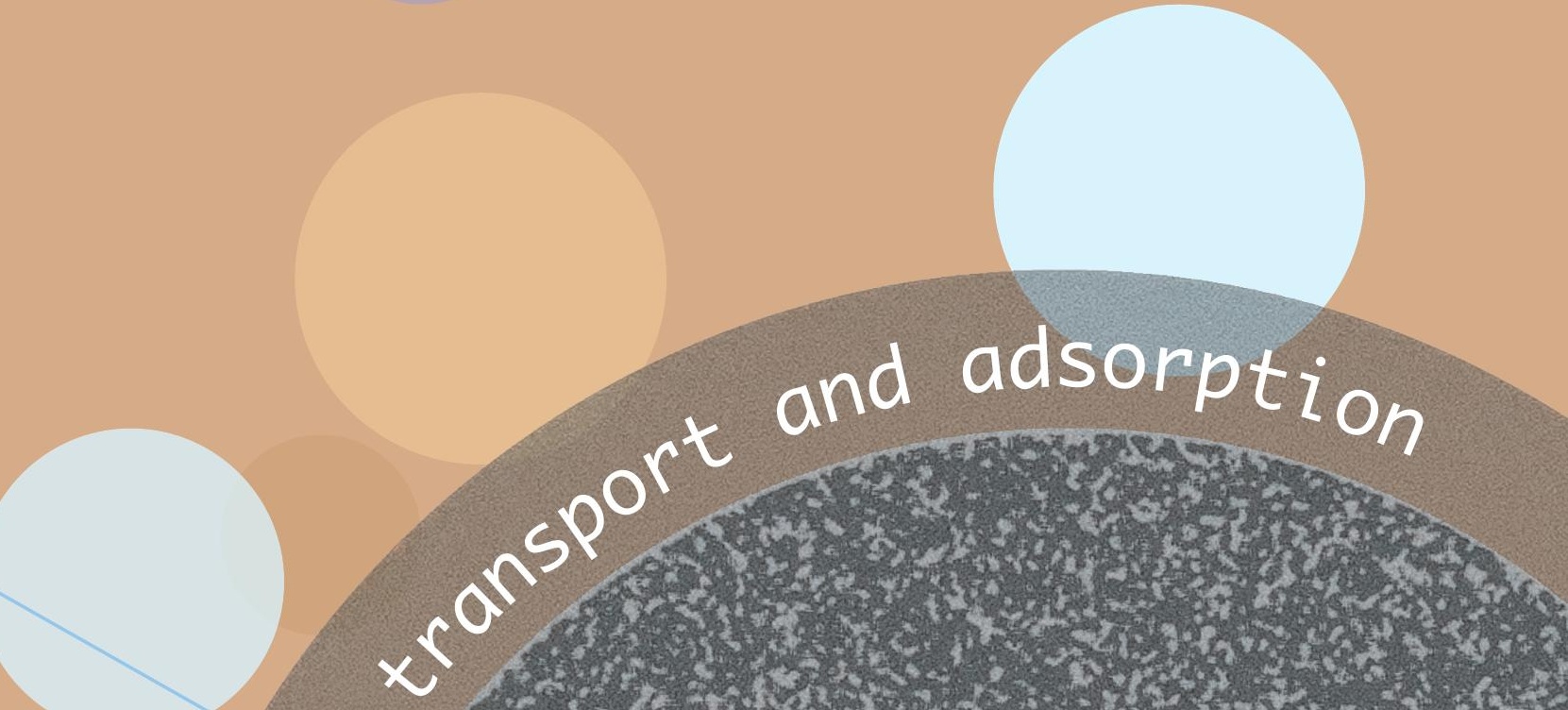

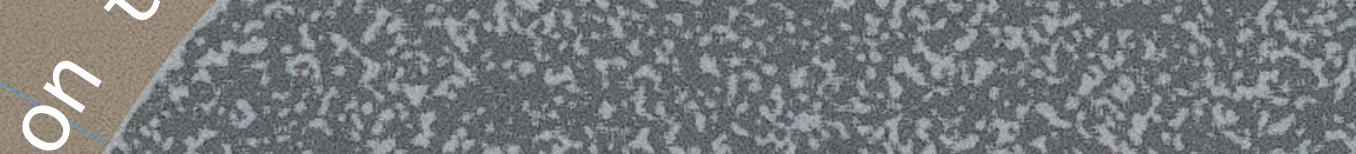

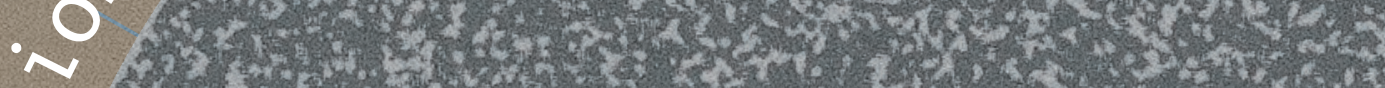

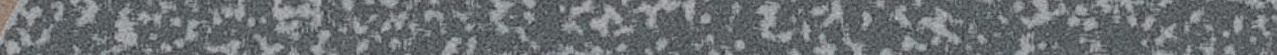
Desalination

With porous

. electrodes 空

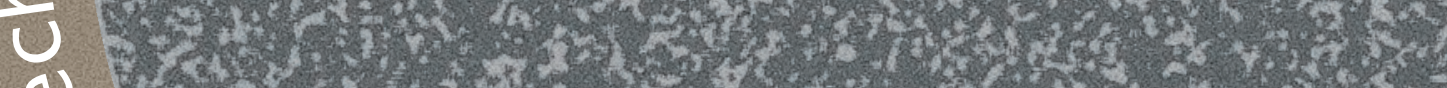

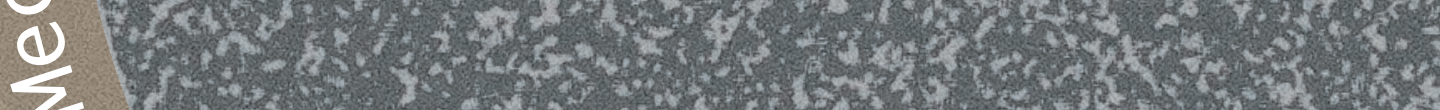
$\sum$ r.t. Hovke Eabele Dykstra 



\section{Desalination}

\section{with porous electrodes}

mechanisms of ion transport and adsorption 


\section{Thesis committee}

\section{Promotor}

Prof. Dr A. van der Wal

Special Professor Electrochemical Water Treatment

Wageningen University \& Research

\section{Co-promotor}

Dr P.M. Biesheuvel

Principal Scientist

Wetsus, European Centre of Excellence for Sustainable Water Technology, Leeuwarden

\section{Other members}

Prof. Dr F.A.M. Leermakers, Wageningen University \& Research

Prof. Dr M.E. Suss, Technion, Haifa, Israel

Prof. Dr R.G.H. Lammertink, University of Twente, Enschede

Prof. Dr L.C. Rietveld, Delft University of Technology

This research was conducted under the auspices of the Wageningen Institute for Environment and Climate Research 


\title{
Desalination \\ with porous electrodes \\ mechanisms of ion transport and adsorption
}

\author{
Jouke E. Dykstra
}

\section{Thesis}

submitted in fulfilment of the requirements for the degree of doctor

at Wageningen University

by the authority of the Rector Magnificus,

Prof. Dr A.P.J. Mol,

in the presence of the

Thesis Committee appointed by the Academic Board

to be defended in public

on Friday 15 June 2018

at 4 p.m. in the Aula. 
Jouke E. Dykstra

Desalination with porous electrodes - mechanisms of ion transport and adsorption, 194 pages.

PhD thesis, Wageningen University, Wageningen, the Netherlands (2018)

With references, with summary in English

ISBN 978-94-6343-833-9

DOI https://doi.org/10.18174/443551 
Foar heit

Ta oantinken oan mem 



\section{Contents}

1 Introduction $\quad \mathbf{1 1}$

1.1 Capacitive Deionization . . . . . . . . . . . . . . . . . . 12

1.2 Membrane Capacitive Deionization . . . . . . . . . . . . 15

1.3 Theory ...................... 16

1.3.1 Ion adsorption . . . . . . . . . . . . . 17

1.3 .2 Ion transport . . . . . . . . . . . . . . . . . . 19

1.3.3 Faradaic reactions . . . . . . . . . . . . 20

1.4 Process design . . . . . . . . . . . . . . . . . . 21

1.4.1 Operational mode: constant current vs. constant voltage . 22

1.4 .2 Cell designs . . . . . . . . . . . . . . . . . . . . . . . . . . . . . . . . 22

1.4.3 Electrode materials . . . . . . . . . . . . . . 24

1.4.4 Process performance metrics .......... . . 24

1.5 Energy consumption . . . . . . . . . . . . . . 25

1.6 Recent scientific and commercial interest in Capacitive Deionization 26

1.7 Outline of this Thesis . . . . . . . . . . . . . . . . . 28

2 Enhanced charge efficiency by increasing the discharge voltage in CDI 31

2.1 Introduction . . . . . . . . . . . . . . . . 33

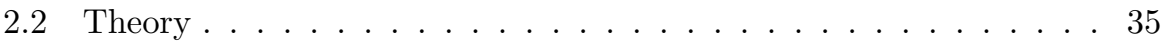

2.2.1 Equilibrium EDL model . . . . . . . . . . . . 35

2.2.2 CDI porous electrode transport theory . . . . . . . . . 38

2.3 Materials and methods . . . . . . . . . . . . . . . . . . . 41

2.3.1 Electrode preparation .............. 41

2.3.2 CDI experiments . . . . . . . . . . . . . 41

2.3.3 Data analysis to derive EDL-properties . . . . . . . . . 43 
2.4 Results and discussion . . . . . . . . . . . . . . . . . 44

2.5 Conclusions . . . . . . . . . . . . . . . . . 51

3 Resistance identification and rational process design in MCDI 53

3.1 Introduction . . . . . . . . . . . . . . . . . 55

3.2 Theory. . . . . . . . . . . . . . . 56

3.2.1 Porous carbon electrodes . . . . . . . . . . . . 58

3.2.2 Spacer channel . . . . . . . . . . . . . . . 59

3.2.3 Membranes ...................66 61

3.3 Characterization: electrode salt adsorption, electronic resistances and spacer properties . . . . . . . . . . . . . . . . . 62

3.3.1 Electrode salt adsorption . . . . . . . . . . . . . 62

3.3.2 Electronic resistances and spacer properties . . . . . . . 64

3.4 Verification: charging and discharge dynamics . . . . . . . . . . . 68

3.5 Prediction: resistances across the MCDI cell . . . . . . . . . . . . 71

3.6 Improved system design: increased electrode thickness . . . . . . . 73

3.7 Conclusions .................... 76

4 Energy consumption in CDI - comparison of operational modes $\quad 77$

4.1 Introduction . . . . . . . . . . . . . . . . . . . . . 79

4.2 Theoretical framework . . . . . . . . . . . . . . 83

4.3 Energy consumption as function of desalination - comparison of operational modes . . . . . . . . . . . . . . . . . . . . 84

4.4 Optimizing desalination performance as function of desalination and WR - comparing constant voltage and constant current . . . . 86

4.5 Conclusions . . . . . . . . . . . . . . 96

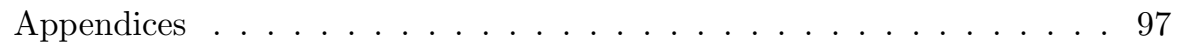

4.A Experimental design for model validation . . . . . . . . . . . 97

5 Dynamics of ion adsorption from mixtures of salts in CDI 101

5.1 Introduction . . . . . . . . . . . . . . . . . . . . 103

5.2 Theory . . . . . . . . . . . . . . . . . . . . 104

5.3 Materials and methods . . . . . . . . . . . . . . . . 107

5.4 Results and discussion . . . . . . . . . . . . . . . . . . . . 109

5.5 Conclusions ............................ 111

6 Effect of dissolved oxygen on carbon electrodes in CDI and MCDI 113

6.1 Introduction . . . . . . . . . . . . . . . . . . . . . 115

6.2 Theory......................... 116

6.2.1 Amphoteric Donnan model . . . . . . . . . . . . . . 118

6.2.2 Change of chemical surface charge . . . . . . . . . . 120 
6.3 Materials and methods . . . . . . . . . . . . . . . 120

6.3.1 CDI experiments . . . . . . . . . . . . 120

6.3.2 MCDI and MCDI-I experiments . . . . . . . . . . . 121

6.3.3 Desalination performance . . . . . . . . . . . . 121

6.4 Results and discussion . . . . . . . . . . . . . . . . . . . 122

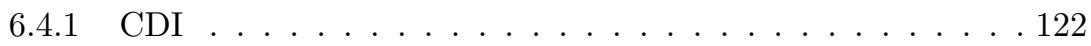

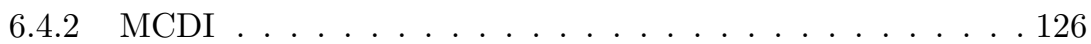

6.5 Conclusions ........................ 126

7 Theory of $\mathrm{pH}$ changes in water desalination by MCDI 129

7.1 Introduction . . . . . . . . . . . . . . . . . . 131

7.2 Theory . . . . . . . . . . . . . . . . . 133

7.2.1 Spacer channel . . . . . . . . . . . . . 134

7.2 .2 Membranes ................... . . 136

7.2.3 Porous carbon electrodes . . . . . . . . . . . . . 137

7.3 Results and discussion . . . . . . . . . . . . . . . . . 139

7.3.1 Equilibrium calculations . . . . . . . . . . . . . . 141

7.3.2 Dynamic calculations . . . . . . . . . . . . . 141

7.4 Conclusions . . . . . . . . . . . . . . . . . 146

Appendices . . . . . . . . . . . . . . . . . . 147

7.A Derivation of auxiliary flux $J_{j, i}^{\text {aux }} \ldots \ldots . \ldots . \ldots . \ldots 147$

7.B Experimental data - Materials and Methods . . . . . . . . . . 148

8 General discussion and conclusions $\quad 151$

8.1 Introduction . . . . . . . . . . . . . . . . . . . . . 152

8.2 Energy consumption and resistance identification . . . . . . . . . 152

8.3 Ion selective adsorption . . . . . . . . . . . . . 156

8.4 Long-term operation and $\mathrm{pH}$ changes . . . . . . . . . . . . . . . . . 157

8.5 Perspectives on technology development . . . . . . . . . . . 158

$\begin{array}{ll}\text { Summary } & 161\end{array}$

$\begin{array}{ll}\text { Bibliography } & 165\end{array}$

$\begin{array}{ll}\text { List of publications } & 181\end{array}$

$\begin{array}{ll}\text { Acknowledgements } & 183\end{array}$

$\begin{array}{ll}\text { About the author } & 189\end{array}$ 



\section{Introduction}

Sections 1.3.1, 1.4.1-1.4.3 and 1.5 have been partially published by the author of this Thesis on Wikipedia (date: August 2, 2013) 


\subsection{Capacitive Deionization}

Capacitive deionization (CDI) is an electrochemical technology to adsorb ions from solution by alternately charging and discharging two electrodes [1-10]. During the charging step, a charging voltage is applied between two electrodes. Consequently, ions are adsorbed into the electrodes, see Fig. 1.1. As a result, feed water is desalinated and fresh water is produced. After the electrodes are saturated with salt, they are discharged and ions are released, resulting in a concentrated effluent stream, often referred to as brine. Also, during discharge, part of the energy consumed during charging can be recovered.
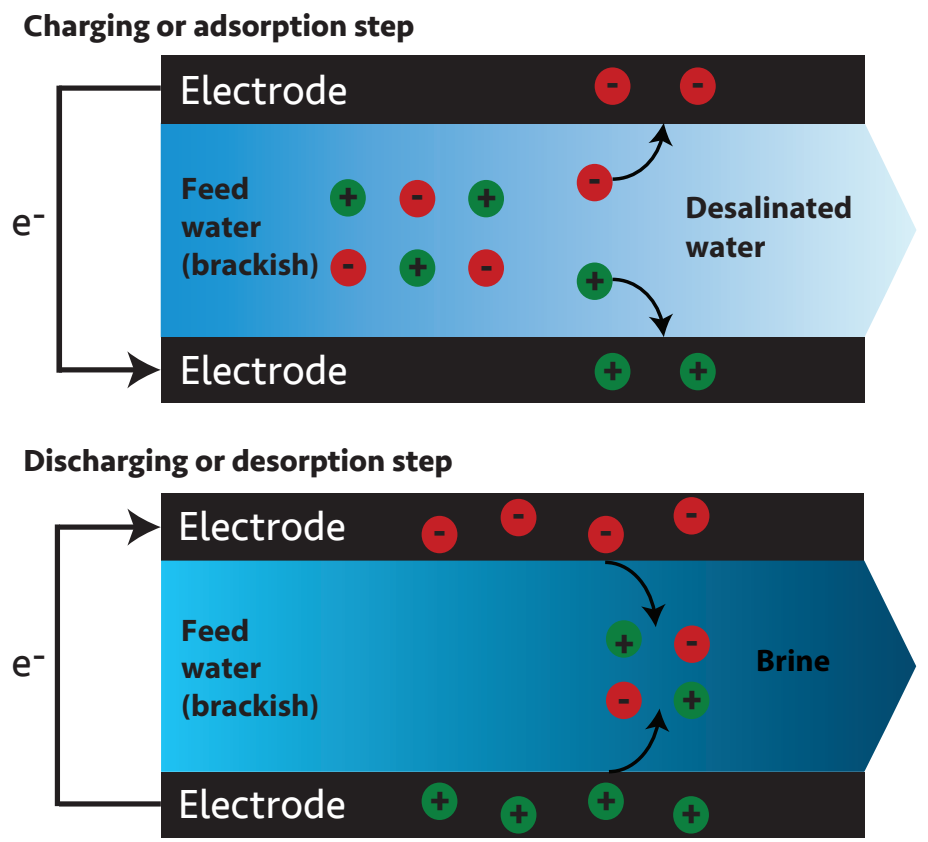

Fig. 1.1: Operation of a Capacitive Deionization cell for water desalination. During the charging or adsorption step, feed water flows into the cell and ions are adsorbed into the electrodes, resulting in a desalinated effluent stream. During the discharge or desorption step, ions are desorbed from the electrodes, resulting in a concentrated effluent stream (brine).

A CDI cell consists of two porous electrodes with a flow (or spacer) channel in between. Electrodes are often made of activated carbon (AC) particles. AC is porous and has a surface area between 1000 and $2000 \mathrm{~m}^{2} / \mathrm{g}$ where ions can be adsorbed [11]. The material is made from natural resources, amongst others from coconut shell, with a raw material cost of $0.50-1.50$ euro $/ \mathrm{kg}$ [12]. 


\section{Broader context: fresh water, a scarce resource}

Fresh water is a scarce resource. Four billion people, two-third of the global population, live, for at least one month per year, in severe water scarcity conditions [13]. The World Economic Forum listed in 2015, water crises as the global risk with the highest impact [14].

One route to combat water scarcity is the development and application of technologies to treat wastewater for re-use, and to produce fresh water from saline water sources. As $97.5 \%$ of water present in reservoirs is saline, water desalination can contribute to solving scarcity problems [15-17].

Desalination technologies separate saline feed water into two streams: a desalinated (fresh) and a concentrated (brine) stream. Existing technologies can be divided into two categories: thermal and membrane-based processes [18]. Thermal desalination is a process based on the evaporation of water: saline water is heated to the boiling point, water evaporates and is condensed as fresh water [19]. Membrane-based processes can be sub-divided based on the driving force used for desalination, which can be pressure or electrical current. Currently, reverse osmosis (RO), a process using pressure as driving force, dominates the desalination market $[16,17,20]$. In RO, membranes are used that only allow the transport of water and block salt and other dissolved matter. By exerting a high pressure on the saline water, water permeates through the membranes, and a fresh water stream is obtained $[18,19,21,22]$.

Desalination is a capital- and energy-intensive process [16, 20, 23]. The energy costs of $\mathrm{RO}$ are in the range of $1-4 \mathrm{kWh} / \mathrm{m}^{3}[23,24]$, and of thermal desalination in the range of $15-25 \mathrm{kWh} / \mathrm{m}^{3}$ [25]. Furthermore, membranebased processes require the use of chemicals to prevent growth of microorganisms on the membranes and to prevent scaling. Residuals of these chemicals are discharged, with the brine, and especially antiscalants can harm the marine environment $[17,24,26]$. The high capital and operational costs of incumbent desalination technologies drive the development of new technologies, as well as the optimization of currently used technologies.

Currently, there is a growing scientific and commercial interest in CDI, an emerging desalination technology. CDI is an electrically driven process: by applying an electrical field between two porous electrodes, salts are adsorbed into the micropores in the electrodes. Zhao et al. [27] found that the energy consumption of desalination by CDI can be lower than RO when the salt concentration of the feed water is below $30 \mathrm{mM}(2 \mathrm{~g} / \mathrm{L})$. Although energy 
consumption is not the only criterion to select a desalination technology for, this finding is an important driver for technology providers and scientists to further develop the technology.

From an application point of view, several aspects are important in the choice of a desalination technology: low energy consumption, stable longterm operation with a minimum of maintenance, and low costs. Furthermore, there is an increasing interest to apply desalination methods to remove specific charged compounds from aqueous streams, for instance, the removal of $\mathrm{NO}_{3}{ }^{-}$, a harmful compound, when present in high concentrations in e.g. drinking water [28]. In this Thesis, we will, after introducing CDI technology in the following sections, address several of these aspects to investigate the suitability of CDI as a method of desalination.

In carbon electrodes, ions are adsorbed into electrical double layers (EDLs), see Fig. 1.2, which are formed in the micropores inside the carbon particles [2932]. These EDLs contain three types of charge: electronic, chemical and ionic. Electronic charge is stored in the solid phase of the material, the chemical charge is stored in the form of chemical groups present at the surface of the material, while the ionic charge is present in the form of ions stored in the diffuse layers of the carbon [33]. The sum of these three types of charge is by definition equal to zero, which means that the overall EDL is charge neutral.

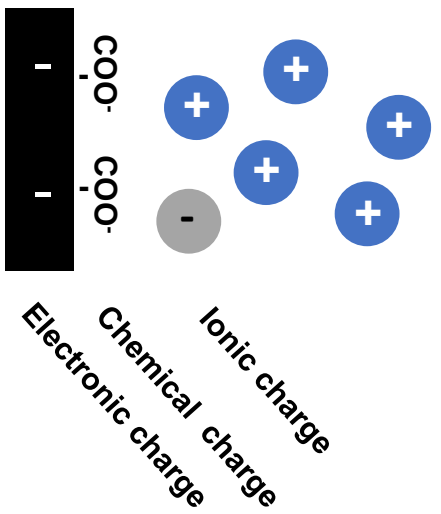

Fig. 1.2: Schematic overview of an electrical double layer. Electronic charge, stored in the electrode, is charge compensated by chemical charge at the surface of the electrode, and by ionic charge, which is stored in the diffuse layer.

So, how does adsorption of ions in EDLs occur? Upon applying a charging voltage over two electrodes, electrical current (electrons) runs from one electrode to the other. The electrons transferred into the electrodes are stored in EDLs, and 
in order to keep EDLs electroneutral, the electronic charge needs to be compensated by chemical or ionic charge. Let us, for now, assume charge compensation takes place by a change in ionic charge (i.e., the amount of chemical charge does not change during operation). This means that for each extra electron stored in the electrode, either a counterion (which is a cation for the cathode and an anion for the anode) is adsorbed, or a co-ion (which is an anion for the cathode and a cation for the anode) is expelled. Whereas adsorption of counterions is desired, desorption of co-ions is unwanted, and results in a charge efficiency of the entire cell, which is the ratio of salt adsorbed over electrons transported, below unity (the theoretical maximum value of charge efficiency is unity).

\subsection{Membrane Capacitive Deionization}

To avoid desorption of co-ions from the electrodes into the flow channel, a cation exchange membrane (CEM) can be placed in front of the cathode, and an anion exchange membrane (AEM) in front of the anode, see Fig. 1.3 [34-38]. These ion exchange membranes (IEMs) allow the passage of counterions, while they block the transport of co-ions. Consequently, membranes increase charge efficiency of CDI to a value close to unity. The CDI technology including membranes in the cell configuration, is referred to as Membrane Capacitive Deionization (MCDI).

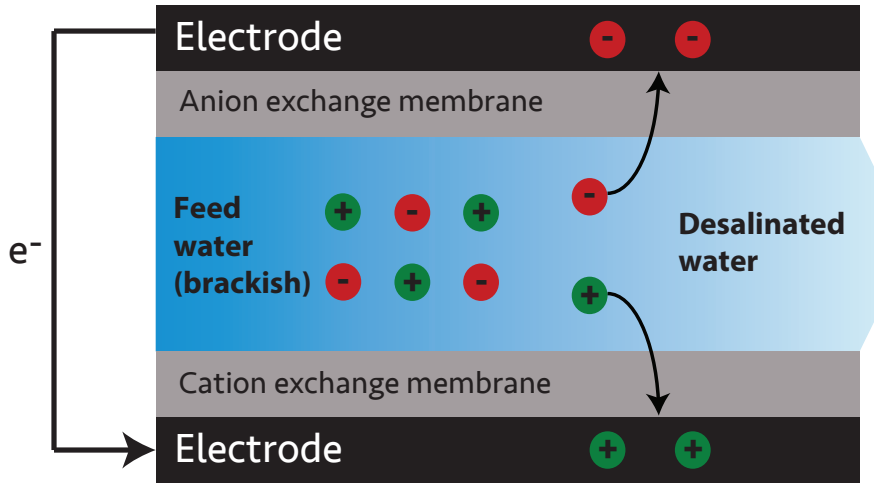

Fig. 1.3: Schematic of a Membrane Capacitive Deionization cell.

The extent to which IEMs block the passage of co-ions, is dependent on the thickness of the membranes, see Fig. 1.4. Clearly, we observe an increase of the thickness from approximately 0.05 to $30 \mu \mathrm{m}$ goes with an increase of charge efficiency and salt adsorption, due to an increase of the selectivity of the membranes. Consequently, the energy consumption (with and without energy recovery during discharge) decreases, and is at minimum if the membrane thickness is between 


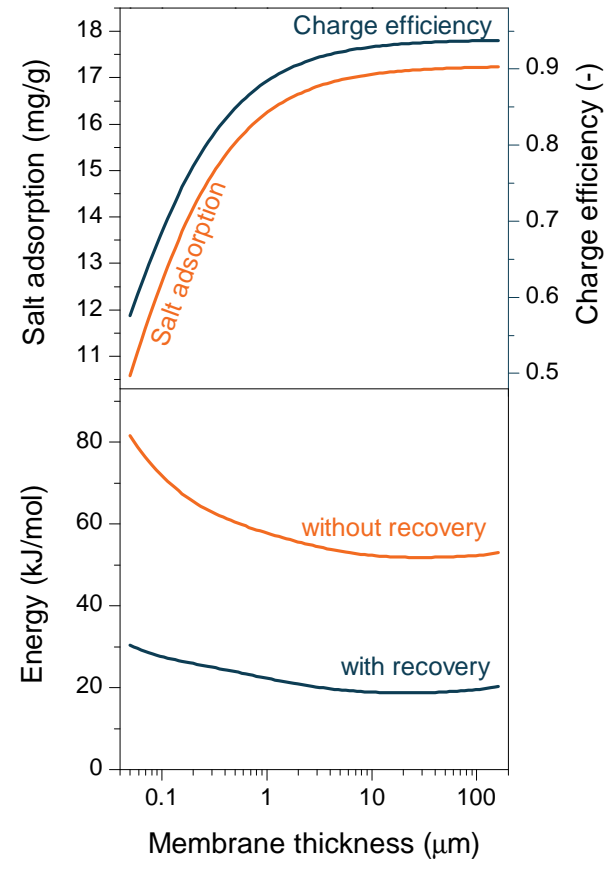

Fig. 1.4: Increasing the membrane thickness in MCDI results in a higher salt adsorption and charge efficiency, and lower energy consumption (with and without considering energy recovery during discharge), and is, in the presented case, at minimum if the thickness is between 15 and $30 \mu \mathrm{m}$. Constant current calculations are performed according to theory presented in Chapter 3. Parameter values are the same as used for calculations discussed in Section 3.5.

15 and $30 \mu \mathrm{m}$. Increasing the thickness further results in an increase of energy consumption, due to an increase of ionic resistances across the membrane, which we will further discuss in Chapter 3.

\subsection{Theory}

To describe CDI theoretically, we distinguish the following processes in the porous carbon electrodes: ion adsorption and ion transport. We consider that these processes occur in two different types of pores in the carbon electrode, see Fig. 1.5:

- macropores: interparticle pores that serve as transport highways for ions;

- micropores: intraparticle pores where EDLs are formed and ions are stored.

Besides macro- and micropores, in Chapters 2 and 3 we include mesopores in the theory, which we will discuss in Section 1.3.1 and in more detail in Section 2.3.3. 


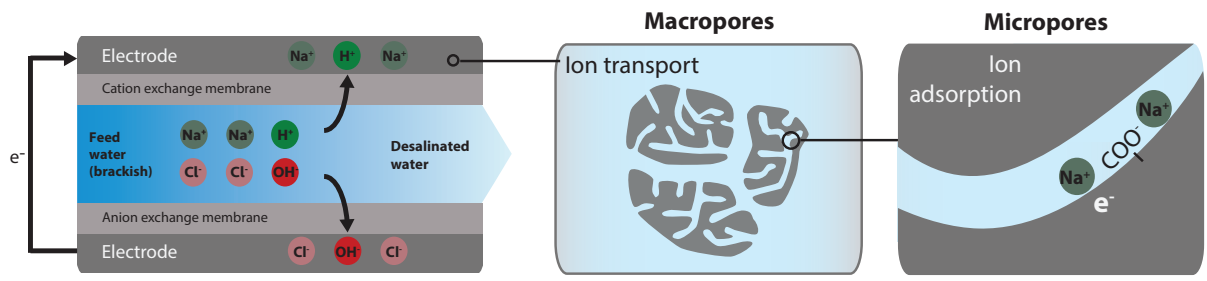

Fig. 1.5: Schematic overview of ion adsorption coupled with ion transport theory in a CDI electrode.

Please note that, in our theoretical work, macro-, meso-, and micropores are defined according to their functional performance, see Refs. [39-41], which is different from the definitions introduced in Section 1.4.3 [42].

To model ion adsorption, we use EDL theory. EDL theory relates the concentration of ions in the micropores to the concentration in bulk solution, or to the concentration in the macropores. To model the dynamics of ion adsorption, we also consider transport of ions from the spacer channel, across the membranes (in MCDI), into the electrodes, towards the micropores. We will discuss both ion adsorption and ion transport theory in the following sections.

\subsubsection{Ion adsorption}

To describe ion adsorption in EDLs, the Gouy-Chapman-Stern theory is often used [43-47]. This theory dates back to 1924 and considers three layers, see Fig. 1.6: the carbon surface, Stern layer and diffuse layer. Electronic charge is stored in the carbon. Ionic charge is stored in the diffuse layer, diffusively distributed close to the surface (carbon). The ion concentration is the highest close to the surface and decreases with distance. The Stern layer is a layer between the diffuse layer and the carbon, and does not contain charge. This layer is often considered to be due to the fact that charged ionic species carry a hydration shell, and therefore the charged ion cannot come infinitely close to the surface.

Although the Gouy-Chapman-Stern theory is often used to calculate ion adsorption in EDLs, it does not predict ion adsorption in CDI accurately [48]. Let us explain this by a simple calculation. The Debye length, $\lambda_{\mathrm{D}}$, which is a measure of the thickness of the diffuse layer, which is for a monovalent salt given by

$$
\lambda_{\mathrm{D}}=\sqrt{\frac{\epsilon_{\mathrm{r}} \cdot \epsilon_{0} \cdot R \cdot T}{2 \cdot F^{2} \cdot c_{\infty}}}
$$

where $\epsilon_{\mathrm{r}}$ is the dielectric constant, $\epsilon_{0}$ the permittivity of free space, $R$ the gas constant, $T$ temperature, $F$ the Faraday constant, and $c_{\infty}$ the salt concentration 


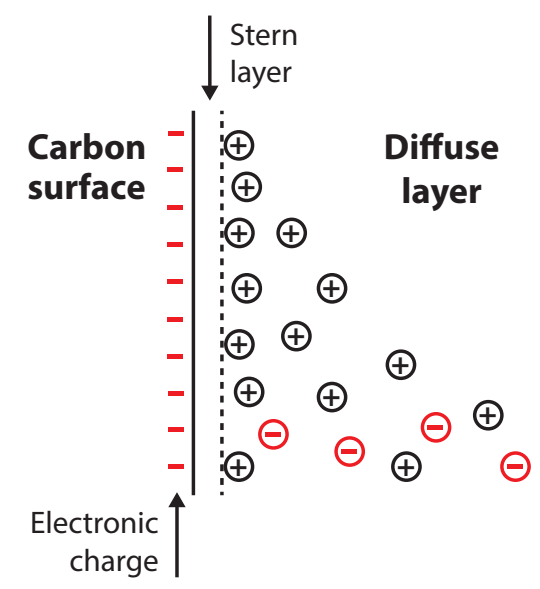

Fig. 1.6: Schematic overview of ion adsorption in EDLs according to the GouyChapman-Stern theory

of the electrolyte. As salt concentrations in CDI are typically low, the Debye length is high. E.g., for an electrolyte with $c_{\infty}=10 \mathrm{mM}$ at $T=293 \mathrm{~K}$, the Debye length is $3.1 \mathrm{~nm}$. As we can consider the diffuse layer to end after 2 or 3 times the Debye length, and as the pore size of the micropores is not more than 2 $\mathrm{nm}$, EDLs are strongly overlapping, a situation which is not described by the Gouy-Chapman-Stern theory.

To describe ion adsorption in strongly overlapping EDLs, we can use the Donnan model, which assumes a constant electrical potential in the micropores [49]. To use the Donnan model for CDI, we make two modifications, which are [42]:

- between the carbon surface and the electrolyte, we include a Stern layer, similar as in the GCS-model;

- we include an attraction term, $\mu_{\text {att }}$, which we use as a fitting parameter to describe ion adsorption in the micropores in the absence of electronic charge, which has been experimentally observed $[42,50]$.

The Donnan model including these two modifications was introduced as the modified Donnan (mD) model in Ref. [47]. In 2014, another modification was introduced, namely that $\mu_{\text {att }}$ is dependent on the total ion concentration in the micropores, and the model was called the "improved modified Donnan" (i-mD) model [49]. In this Thesis, we describe and use the i-mD model in Chapters 2-5.

An important point to note is that the micropore volume, $v_{\mathrm{mi}}$, that we find by fitting the parameters in the $\mathrm{i}-\mathrm{mD}$ model with experimental data for salt adsorption, is less than the micropore volume measured by gas sorption analysis, $v_{G S A}$. 
In Chapter 2 we suggest to call this difference mesoporosity, $v_{\text {meso }}$. In the mesopores, like in the nearby macropores, we assume equal concentrations of cations and anions (for a 1:1 salt), at the same concentration as in the nearby macropores. The only difference with the macropores is that the ions in the mesopores do not contribute to ion transport across the electrode.

Furthermore, we note that, with both the $\mathrm{mD}$ and $\mathrm{i}-\mathrm{mD}$ models, the presence of chemical surface charge, as indicated in Fig. 1.2, has not been considered. This changed with the introduction of the amphoteric Donnan (amph-D) model in 2015, which includes two modifications compared to the i-mD model [51]. The first modification is the introduction of chemical surface charge. Thus, the amphoteric Donnan model considers three types of charge: electronic, chemical and ionic. Secondly, because of the introduction of chemical surface charge, which predicts the adsorption of ions in micropores under conditions of zero electronic charge, we do not have to consider $\mu_{\text {att }}$ anymore, which was a fitting term to start with. As shown in Fig. 1.7, the amph-D model considers two types of groups contributing to chemical surface charge, namely groups with positive charge, basic groups, which are often depicted in the form of $\mathrm{H}^{+}$, and groups with negative charge, acidic groups, depicted in the form of $\mathrm{COO}^{-}$.

An important improvement of the amph-D model compared to the $\mathrm{i}-\mathrm{mD}$ model is that the value for $v_{\mathrm{mi}}$, that we find by fitting the amph-D model to experimental data for salt adsorption, is now much closer to the micropore volume measured by gas sorption analysis, $v_{G S A}$. Therefore, we no longer consider mesopores in the amph-D model, which we only introduced in the i-mD model to attribute the difference between $v_{G S A}$ and $v_{\mathrm{mi}}$ to a parameter.

In this Thesis, we describe and use the amph-D model in Chapters 6 and 7.

\subsubsection{Ion transport}

To describe CDI dynamically, we couple ion adsorption with ion transport theory. The first papers published on this topic date back to 1963 and describe transmission line theory [52-54], which assumes a constant salt concentration in the macropores of the electrodes. To model CDI, this assumption was relaxed and the theory was extended by a description of ion transport across the electrode $[55,56]$.

To include a more realistic EDL model than included in the transmission line theory, porous electrode theory was derived in Refs. [39-41], which couples the (i-)mD model to ion transport theory. In this Thesis, we use and describe porous electrode theory in Chapters 2-5.

Besides modeling ion transport in porous electrodes, we also model ion transport in the spacer channel and through ion exchange membranes in Chapters 3 

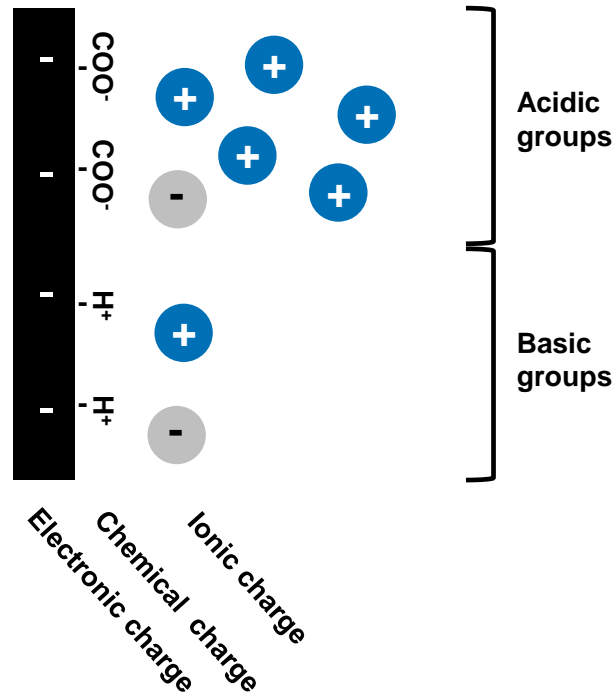

Fig. 1.7: Schematic overview of ion adsorption in EDLs according to the amphoteric Donnan model.

and 7. Furthermore, in Chapter 7 we derive a model combining ion transport with water self-ionization, $\mathrm{H}_{2} \mathrm{O} \rightleftarrows \mathrm{H}^{+}+\mathrm{OH}^{-}$.

\subsubsection{Faradaic reactions}

Until now, we discussed ion adsorption in CDI as a capacitive process. A capacitive process, on the one hand, does not involve the transfer of charge across the electrode-solution interface [57]. On the other hand we have faradaic reactions, see Fig. 1.8A, which form the basis of many electrochemical technologies, such as batteries, fuel cells and electrolysis cells. These faradaic reactions do involve charge transfer across the electrode-solution interface, which result in a chemical change of the compounds in solution or the electrode material. Perhaps the most well-known faradaic reaction is water splitting (or water electrolysis), see Fig. 1.8B, where we have:

- the oxidation of water at the anode $\left(2 \mathrm{H}_{2} \mathrm{O} \rightleftarrows 4 \mathrm{H}^{+}+\mathrm{O}_{2}+4 \mathrm{e}^{-}\right)$, and thus the faradaic transfer of electrons from solution $\left(\mathrm{H}_{2} \mathrm{O}\right)$ into the electrode (anode);

- the transfer of electrons from anode to cathode;

- the reduction of water at the cathode $\left(4 \mathrm{H}_{2} \mathrm{O}+4 \mathrm{e}^{-} \rightleftarrows 2 \mathrm{H}_{2}(\mathrm{~g})+4 \mathrm{OH}^{-}\right)$, and thus the faradaic transfer of electrons from the electrode (cathode) into solution $\left(\mathrm{H}_{2} \mathrm{O}\right)$. 


\section{A) capacitive vs faradaic}

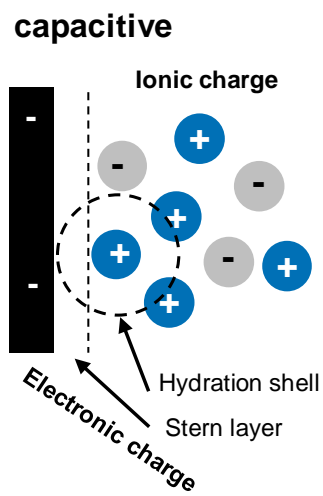

\section{B) example of a faradaic process}

faradaic

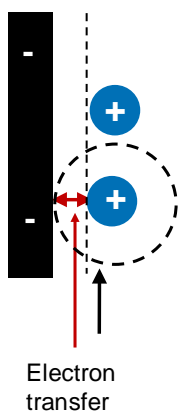

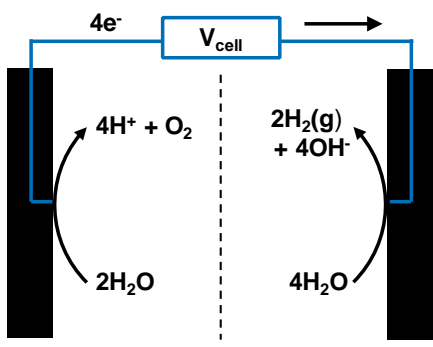

Fig. 1.8: Capacitive and faradaic processes. Whereas a capacitive process results in storage of electronic charge in the solid phase, and consequently, adsorption of ions in the diffuse layer, a faradaic process involves the transfer of an electron from the solid phase to the electrolyte.

Although CDI is based on a capacitive process, faradaic reactions may also occur [58-65]. These faradaic reactions can be unfavorable in CDI, as they

- reduce charge and energy efficiency of the process (electrons involved in faradaic reactions do not contribute to ion adsorption in EDLs);

- can result in unwanted pH changes of the effluent solution, as some reactions involve the production or consumption of protons or hydroxyl ions, which can result in precipitation of salt on membranes or into electrodes, and thereby affect the long-term stability of the cell [64, 66-68];

- can result in oxidation of carbon - often the main electrode material in CDI - and thereby also affect the stability of the CDI desalination process [63-65].

In Chapter 7 we describe the Frumkin-Butler-Volmer theory [40, 69, 70], which we use to model combined capacitive-faradaic processes to understand the cause of $\mathrm{pH}$ fluctuations in MCDI.

Table 1.1 summarizes which theory is used in each Chapter of this Thesis.

\subsection{Process design}

In this section, we discuss several considerations for the design of a CDI process: operational mode (constant current vs. constant voltage), cell designs, electrode materials, and process performance metrics. 


\subsubsection{Operational mode: constant current vs. constant voltage}

In Section 1.1 we described that, upon applying a voltage over two electrodes, an electrical current runs between anode and cathode, resulting in an electronically positively charged anode, and a negatively charged cathode. Implicitly, we suggested that the cell is operated by controlling the voltage at constant value. However, one can also control the current at constant value. We describe the main differences between both operational modes.

\section{Constant voltage}

During the adsorption step in (M)CDI operated in constant voltage mode, the effluent salt concentration rapidly decreases, and thereafter, the effluent salt concentration increases again. This can be explained by the fact that EDLs are uncharged at the beginning of an adsorption step, which results in a high voltage over the two electrodes, and consequently in a high driving force for ions to adsorb into the EDLs. When more ions are adsorbed in the EDLs, the EDL potential increases and the remaining potential difference between the electrodes, which drives the ion transport, decreases. Because of the decreasing ion removal rate, the effluent concentration increases again [71].

\section{Constant current}

As the applied electrical current is equal to the ionic current in a (M)CDI cell, constant current mode allows a better control, compared to constant voltage, on the ion transport from spacer into electrodes, and thus on the effluent salt concentration. However, for a stable effluent salt concentration membranes should be incorporated in the cell design (MCDI), as the electric current does not only induce counterion adsorption, but co-ion desorption as well [71].

Although one cannot control current and voltage at the same time, combinations of both operational modes are possible during a desalination cycle, e.g., one can charge the cell at constant current, and discharge at constant voltage.

\subsubsection{Cell designs}

Many different cell designs have been reported in literature [2], of which we discuss the most distinct designs.

\section{Flow-by}

Electrodes are placed in a stack with a thin spacer layer in between, through which the water flows, see Figs. 1.1 and 1.3. This is by far the most commonly used design, which we used for all work in this Thesis. 
Flow-through

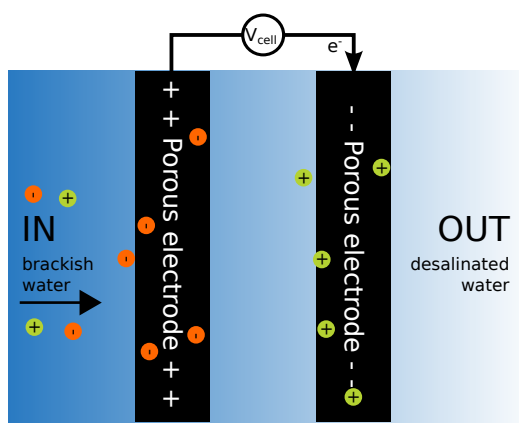

Flow-electrode

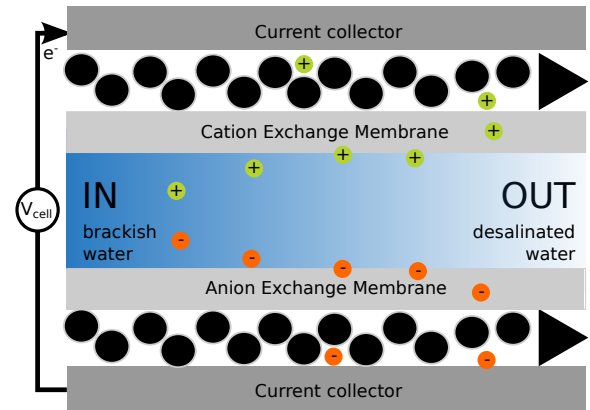

Fig. 1.9: Schematic overview of flow-through and flow-electrode CDI designs.

\section{Flow-through}

In this mode, feed water flows straight through the electrodes, i.e. the water flows directly through the interparticle pores of the porous carbon electrodes, see Fig. 1.9.

\section{Flow-electrode}

This design is comparable to flow-by mode with membranes in front of both electrodes, but instead of having solid electrodes, a carbon suspension (slurry) flows between membranes and current collector, see Fig. 1.9. A voltage is applied between both channels of flowing carbon slurries, and ions are adsorbed from the water into the carbon slurries. Since the slurries flow, electrodes do not saturate and therefore this cell design can be used for the desalination of water with high salt concentrations (e.g. sea water, with salt concentrations of approximately $30 \mathrm{~g} / \mathrm{L})$. A discharge step is not necessary; the carbon slurries are, after leaving the cell, mixed together and ions desorb. Thereafter, the carbon slurry can be separated from the concentrated salt water, and the slurry can be reused $[5,7,72-$ $79]$.

\section{Wires}

Fresh water can be produced continuously in a modified CDI configuration where rod-shaped anode and cathode electrode pairs are not fixed in space, but move cyclically from one stream, in which the voltage is applied and salt is adsorbed, to another stream, where the voltage is reduced and salt is released $[80,81]$. 


\subsubsection{Electrode materials}

In this work, we study electrodes made of carbon material. Regarding the structure of the material, there are several considerations. As a high salt electrosorption capacity is important, the specific surface area of the carbon accessible for ions should be large. Furthermore, the material should be stable and no chemical degradation of the electrodes should occur in the voltage window applied for CDI. The ions should be able to move fast through the pore network of the carbon and the electronic conductivity of the carbon should be high [42].

To classify pore sizes, definitions of the International Union of Pure and Applied Chemistry (IUPAC) can be used [42, 82, 83]. IUPAC defines the pores according to size as follows:

- Macropores: larger than $50 \mathrm{~nm}$;

- Mesopores: between 2 and $50 \mathrm{~nm}$;

- Micropores: smaller than $2 \mathrm{~nm}$.

Nowadays, activated carbon (AC) is the commonly used material, as it is the most cost efficient option and it has a high specific surface area. It can be made from natural or synthetic sources. Other carbon materials used in CDI research are, for example, ordered mesoporous carbon, carbon aerogels, carbide-derived carbons, carbon nanotubes, graphene, and carbon black [42].

\subsubsection{Process performance metrics}

To evaluate the performance of CDI cells, various metrics are used:

- Salt adsorption capacity (SAC): salt adsorption per amount of electrode material, to indicate salt adsorption performance of the electrodes [84]. The value is not only dependent on the electrode material under study, but also on the charging voltage and the electrolyte (type of salt and respective concentration).

- Charge efficiency $(\Lambda)$ : ratio of salt adsorbed over electrons transferred between the electrodes [48]. The value is dependent on the Stern layer capacitance of the electrode material, the charging voltage, and the electrolyte.

- Water recovery (WR): ratio of volume of desalinated water produced over the total amount of water fed into the system. For example, a system with $\mathrm{WR}=0.5$ and a total feed water stream of $4 \mathrm{~m}^{3} / \mathrm{h}$ produces $2 \mathrm{~m}^{3} / \mathrm{h}$ desalinated water and $2 \mathrm{~m}^{3} / \mathrm{h}$ brine. We define the volume of desalinated water as that when the salt concentration in the desalinated water, $c_{\text {desalinated }}$, is lower than the feed water, $c_{\text {feed }}$. 
- Average salt adsorption rate (ASAR): salt adsorption per cycle divided by the cycle time, which is the total duration of an adsorption and desorption step [35].

- Desalination $(\Delta c)$ : average salt concentration difference between feed water and desalinated water, $\Delta c=\frac{1}{t_{\mathrm{ch}}} \int\left(c_{\text {feed }}-c_{\text {desalinated }}\right) \mathrm{d} t$, where $t_{\mathrm{ch}}$ is the duration of a charging step. Desalination relates to ASAR according to $\operatorname{ASAR}=\Delta c \cdot \Phi_{\mathrm{v}} \cdot \mathrm{WR} \cdot \frac{M_{\mathrm{w}, \text { salt }}}{M_{\text {elec }}}$ under the assumption that $\Phi_{\mathrm{v} \text {, desalinated }}=$ $\Phi_{\mathrm{v}, \text { conc }}=\Phi_{\mathrm{v}, \text { feed }}$, where $\Phi_{\mathrm{v}, i}$ is the flow rate of flow $i\left(\mathrm{~m}^{3} / \mathrm{s}\right)$. Parameter $M_{\mathrm{w}, \text { salt }}$ refers to the molar mass of the salt, and $M_{\text {elec }}$ to the total mass of the electrodes used in the cell.

- Energy consumption: energy consumed during desalination, which is often expressed per mol of salt removed from feed water or per volume of desalinated water produced. As (M)CDI allows energy recovery during the discharge step, the energy consumption can be expressed with or without accounting for energy that can (potentially) be recovered.

For (industrial) system design, the metrics that come into play are ASAR, WR, and energy consumption (with and/or without recovery). Designing a system requires a trade-off between these metrics: increasing ASAR (and thereby decreasing the amount of materials required to build the system for a given production objective) and/or increasing WR result in an increase of energy consumption.

\subsection{Energy consumption}

The energy consumption in CDI depends on many factors and is mainly dependent on system design choices, which we discuss in Chapter 3. The thermodynamic minimum energy consumption of a desalination process can be calculated easily. This minimum energy consumption is due to the fact that desalination involves demixing of ions, which decreases the entropy of the system and therefore requires energy input. This energy input, the Gibbs free energy, $\Delta G$, can be calculated using

$$
\Delta G=G_{\text {desalinated }}+G_{\text {conc }}-G_{\text {feed }}
$$

with $G_{f}$ the Gibbs energy of flow $f$, where $f$ can be replaced by "desalinated" for the desalinated water flow, "conc" for the concentrated flow (or "brine") and "feed" for the feed flow to the cell. For ideal solutions $G_{f}$ is given by

$$
G_{f}=R \cdot T \cdot \Phi_{\mathrm{v}, f} \sum_{i} c_{f, i} \cdot \ln \left(c_{f, i}\right)
$$

where $i$ runs over all ions present in solution, and where $c_{f, i}$ is the concentration of ion $i$ in flow $f, R$ is the gas constant $\left(8.314 \mathrm{~J} \mathrm{~mol}^{-1} \mathrm{~K}^{-1}\right), T$ temperature $(\mathrm{K})$, and $\Phi_{\mathrm{v}, i}$ the flow rate of flow $f\left(\mathrm{~m}^{3} / \mathrm{s}\right)$. 


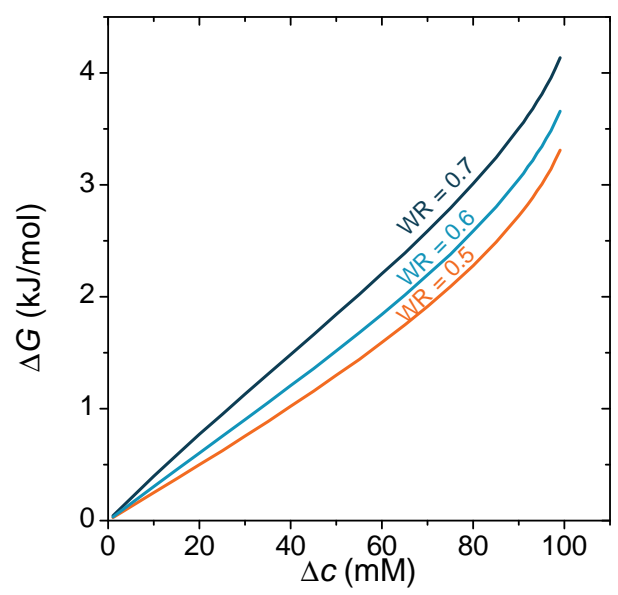

Fig. 1.10: Thermodynamic minimum energy consumption, $\Delta G$, as function of desalination, $\Delta c$, and water recovery, WR. The salt concentration in the feed flow, $c_{\text {feed }}$, is $100 \mathrm{mM}$. Clearly, increasing $\Delta c$ or WR results in higher $\Delta G$.

Fig. 1.10 shows $\Delta G$ as function of $\Delta c$ for $c_{\text {feed }}=100 \mathrm{mM}$. We observe that, with increasing $\Delta c$ (or ASAR) or WR, the minimum energy consumption for desalination increases. Although the real energy consumption is significantly higher than $\Delta G$, we see in Chapter 4 that the relation between energy consumption, $\Delta c$, and WR is similar.

\subsection{Recent scientific and commercial interest in Capacitive Deionization}

The first study on CDI was published in the early 1960s, titled "electrochemical demineralization of water" authored by G.W. Murphy and co-workers [85-87]. During the decades thereafter, the scientific interest in CDI was relatively small, but around 2010 the topic received a major boost. Nowadays, in 2017, about 100 papers are yearly published, see Fig. 1.11. Recent scientific developments are on electrode materials [11, 33, 51, 88-93], novel cell designs, ranging from desalination with wires [80] to flow electrodes [5, 72, 73], or on new operational modes or ion-selective removal [28, 47, 94-96]. Also, studies are conducted to quantify resistances and energy losses in the CDI cell [4, 97, 98], or to identify parasitic reactions that may result in $\mathrm{pH}$ changes [8, 99-101].

The interest in CDI is not limited to the scientific community; there is also commercial interest. Nowadays, Voltea (Fig. 1.12), EST Water Ltd, Idropan, Atlantis, SionTech, and several other companies, develop (M)CDI technology. 


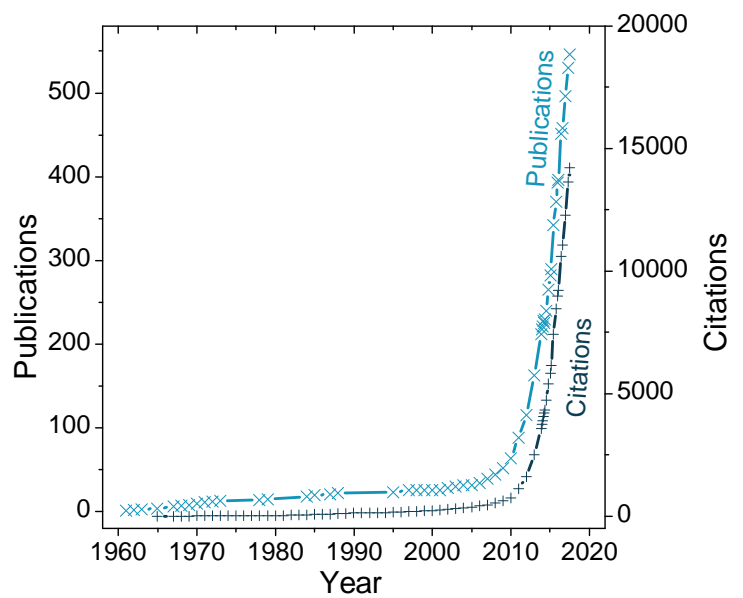

Fig. 1.11: Cumulative number of publications and citations on CDI since the early 1960s. Data is obtained from Web of Knowledge.

Besides desalination of brackish water, as discussed in Section 1.1, there are various other applications of (M)CDI, for instance:

- Water remediation for cooling towers: in industrial processes, cooling towers are used to dissipate heat by evaporation of water. If ions are present in water, the ions can precipitate and result in scaling. To reduce scaling, antiscalents can be used in the recirculation water, which is commonly done, or the water can be desalinated, for example by (M)CDI [34, 102].

- Water softening: to avoid scaling problems in washing machines, dishwashers, boilers, heat exchangers and many other devices, removal of hardness ions, such as $\mathrm{Ca}^{2+}$ and $\mathrm{Mg}^{2+}$, from feed water is necessary. To that end, several desalination methods can be applied, for instance (M)CDI [103].

From an application point of view, several aspects are important. Firstly, stable long-term operation has to be guaranteed. Also, the water recovery, salt adsorption capacity and rate should be high, and the energy costs and capital costs should be low. Furthermore, there is an increasing interest to apply desalination methods to remove specific charged compounds from aqueous streams. In this Thesis, we will address several of these aspects to investigate the suitability of CDI as a method of desalination. 


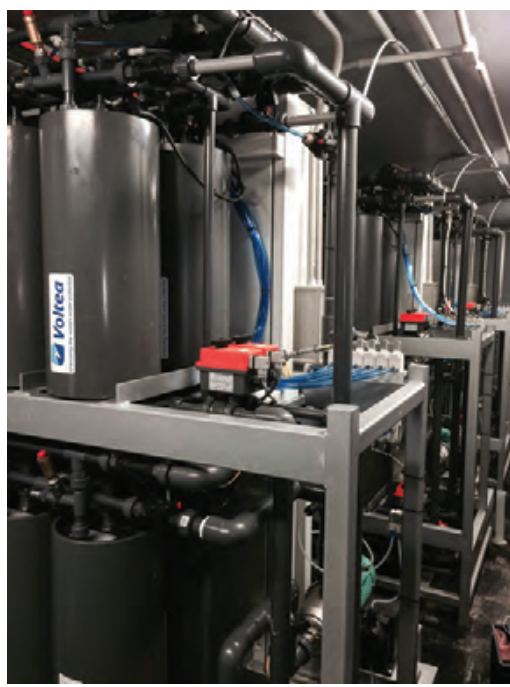

Fig. 1.12: Commercial MCDI system manufactured by Voltea.

\subsection{Outline of this Thesis}

In this Thesis we study the mechanisms of ion transport and adsorption in CDI technology, and we address three topics: I) energy consumption, and resistance identification, II) ion-selective adsorption, and III) long-term operation and $\mathrm{pH}$ changes.

In Chapter 2, we first describe salt adsorption in CDI using the i-mD model. We show that we can increase salt adsorption and energy efficiency by increasing the voltage during discharge, compared to the conventional discharge voltage of $0 \mathrm{~V}$.

In Chapter 3, we combine this i-mD model with transport theory to model the dynamics of ion adsorption in CDI. We verify theory with experimental data and thereafter use the theory to calculate the contribution of different elements of the cell (electrodes, spacer, membranes) to the total cell resistance and energy consumption.

In Chapter 4, we use the theory derived in Chapter 3 to compare the energy consumption of two modes of operation which are frequently used in CDI, constant current and constant voltage. We study energy consumption (with and without energy recovery during discharge) as function of desalination and WR.

Thereafter, we extend the theory to study the dynamics of adsorption of mixtures of salts. Thus, instead of modeling ion transport and adsorption in an electrolyte that contains one monovalent salt, such as $\mathrm{NaCl}$ or $\mathrm{KCl}$, we study 
mixtures, in this case of $\mathrm{NaCl}$ and $\mathrm{KCl}$. We compare theory with experimental data and present the results in Chapter 5 .

In Chapter 6 we apply a new Donnan model, the amph-D model. With this amph-D model, we study the decrease in salt adsorption performance of CDI cells treating water that contains dissolved oxygen. We also explain why (M)CDI cells show a stable performance.

Finally, in Chapter 7, we combine the amph-D model with transport theory. Here, we extend this transport theory to include acid-base equilibrium reactions, in this case water self-ionization $\left(\mathrm{H}^{+}+\mathrm{OH}^{-} \rightleftarrows \mathrm{H}_{2} \mathrm{O}\right)$. Furthermore, we include Frumkin-Butler-Volmer theory to model faradaic reactions. We use this combined theory to obtain mechanistic insight in $\mathrm{pH}$ changes in MCDI.

Table 1.1 summarizes which theory is used in each Chapter of this Thesis. 


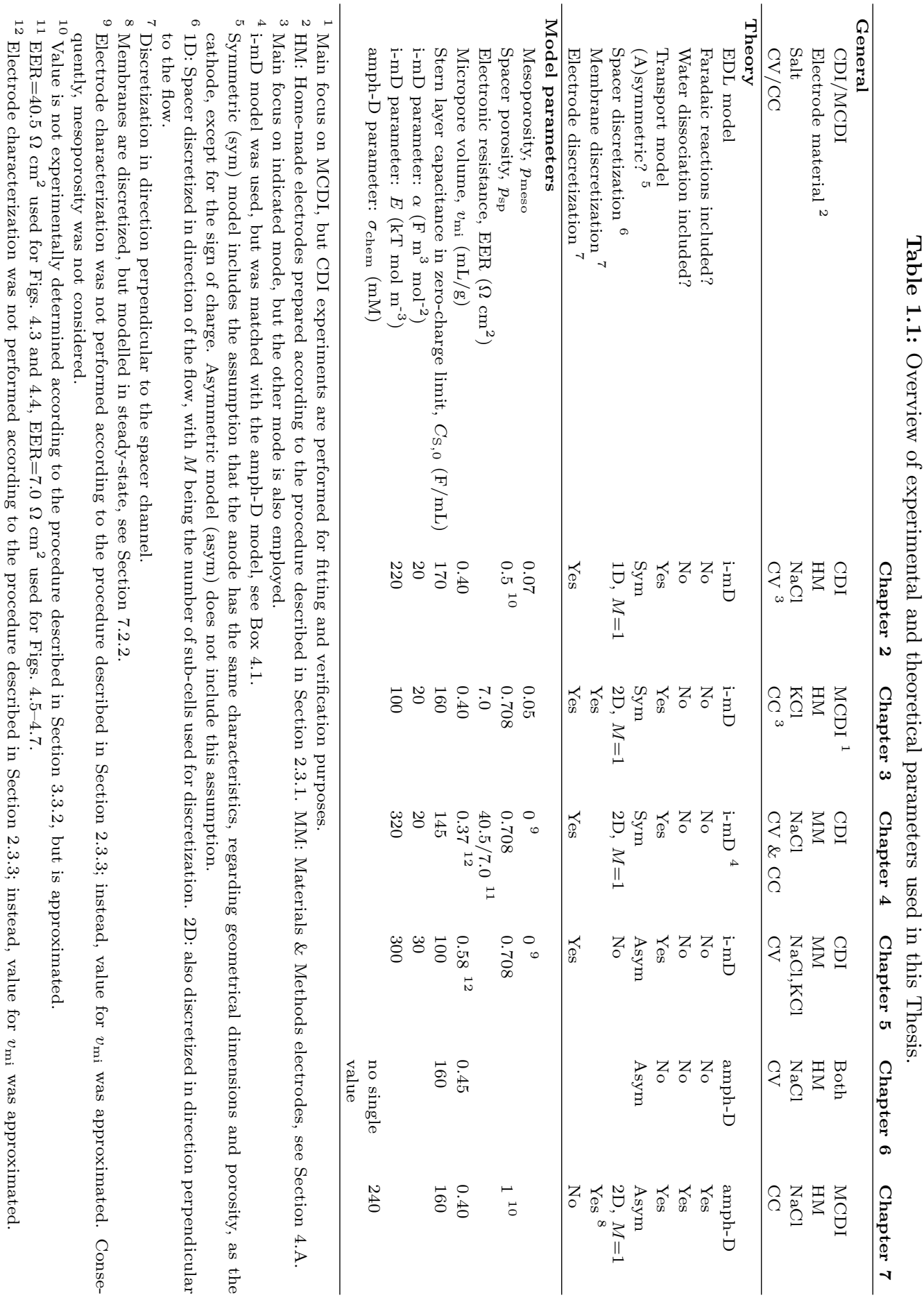




\section{Enhanced charge efficiency by increasing the discharge voltage in CDI}

This Chapter has been published as:

T. Kim, J.E. Dykstra, S. Porada, A. van der Wal, J. Yoon, P.M. Biesheuvel, Enhanced charge efficiency and reduced energy use in capacitive deionization by increasing the discharge voltage, Journal of Colloid and Interface Science 446 (2015) 317-326. 


\section{Abstract}

Capacitive deionization (CDI) is an electrochemical method to adsorb ions from solution by alternately charging and discharging two electrodes in contact with aqueous electrolyte. A key parameter in CDI is the charge efficiency, $\Lambda$, which is the ratio of salt adsorption over charge in a CDI cycle. Values for $\Lambda$ in CDI are typically in the range of $0.5-0.8$, significantly less than the theoretical maximum of unity, due to the fact that not only counterions are adsorbed into the pores of the carbon electrodes, but at the same time co-ions are released. To enhance $\Lambda$, ion-exchange membranes (IEMs) can be implemented. With membranes, $\Lambda$ can be close to unity because the membranes only allow passage for the counterions. Enhancing the value of $\Lambda$ is advantageous as this implies a lower electrical current and (at a fixed charging voltage) a reduced energy use. We demonstrate how, without the need to include IEMs, the charge efficiency can be increased to values close to the theoretical maximum of unity, by increasing the voltage during discharge, with only a small loss of salt adsorption capacity per cycle. In separate constant-current CDI experiments, where after some time the effluent salt concentration reaches a stable value, this value is reached earlier with increased discharge voltage. We compare the experimental results with predictions of porous electrode theory which includes an equilibrium Donnan electrical double layer model for salt adsorption in carbon micropores. Our results highlight the potential of modified operational schemes in CDI to increase charge efficiency and reduce energy use of water desalination. 


\subsection{Introduction}

Capacitive deionization (CDI) is an electrochemical method to adsorb ions from solution by alternately charging and discharging two electrodes in contact with aqueous electrolyte $[11,96,104-110]$. In this process, ions migrate to the electrodes following charge transfer through the external circuit that connects the two electrodes. Within the electrode, ions are stored in the electrical double layers (EDL) which form in the micropores inside the porous carbon particles that compose the electrode [111]. As a consequence, the water flowing through the cell becomes partially desalinated. Generally, the two electrode films are placed parallel to the direction of water flow which runs in between the electrodes through a spacer layer, see Fig. 2.1A. Alternative designs use carbon electrode wires [80], flowable electrode slurries $[7,72-74,76]$ or flow-through electrodes [112]. In addition, electrodes can be chemically modified [88, 113], nanoparticles can be incorporated [114-116], ion-selective coatings can be applied [117, 118], or ionexchange membranes can be placed in front of the electrodes in a modification called Membrane Capacitive Deionization, or MCDI [27, 28, 35, 71, 102, 119-121].

In most of these designs, CDI comprises a repeated cycle of charging (salt adsorption) and discharge (salt release). Discharge of the cell is generally done by short-circuiting the two electrodes (i.e., a discharge voltage of $V_{\text {disch }}=0 \mathrm{~V}$ is applied), where ions are released from the electrodes and a concentrated saline stream is temporarily produced. The two steps of salt adsorption (charging) and desorption (discharge) constitute one full CDI cycle. Charge efficiency $\Lambda$ describes the ratio of salt adsorption (desorption) over the charge transfer [107, 109, 116, 122-124]. The charge efficiency differs between materials and depends on the voltage applied during charging, $V_{\mathrm{ch}}$, and salt concentration [49]. For a typical charging voltage of $V_{\mathrm{ch}}=1.2 \mathrm{~V}$ and for salt concentrations in the range 5-50 $\mathrm{mM}$, values for $\Lambda$ ranging from 0.5 to 0.8 have been experimentally found. Charge efficiency $\Lambda$ increased with $V_{\mathrm{ch}}$, and decreased with salt concentration [36]. Values for $\Lambda$ close to, or beyond, unity have never been reported for CDI. Indeed, a value of $\Lambda$ of unity is considered the theoretical maximum, and would be achieved when one full $\mathrm{NaCl}$ salt molecule is removed for an equivalent electron transfer between the electrodes. The lower values of $\Lambda$ obtained in practice, are disadvantageous as it implies more charge transfer and electrical energy input than is necessary for a given objective of salt removal. However, such sub-optimal values of $\Lambda$ clearly below unity seem to be inherently connected to CDI as a consequence of the structure of the electrical double layer (EDL) in microporous carbons, where the electrical charge is partially compensated by counterion adsorption, and partially by co-ion repulsion (ejection of co-ions from the EDL) [49]. The repulsion of the co-ions results in sub-optimal values of the charge efficiency, i.e. a charge 

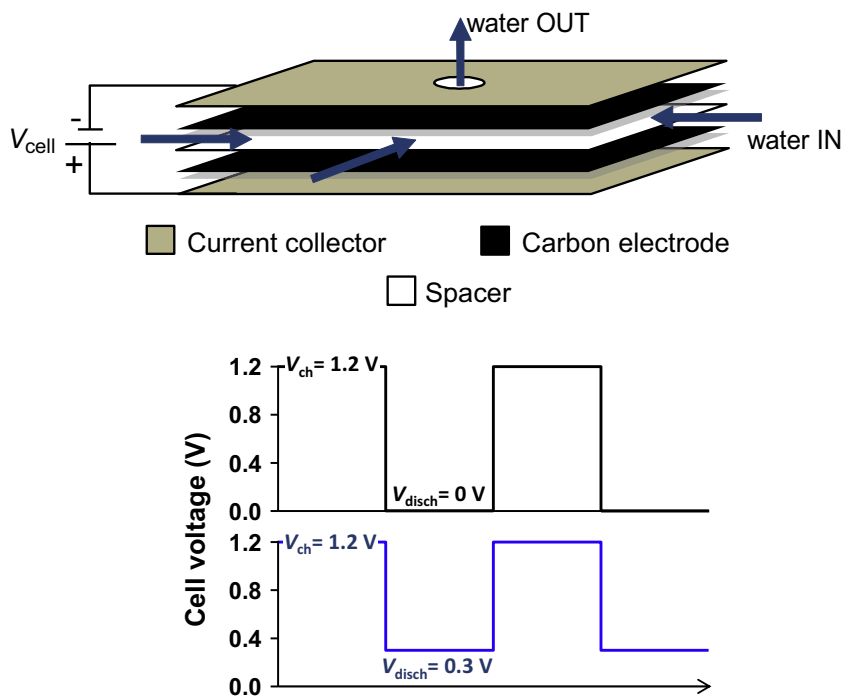

Fig. 2.1: (a) Schematic diagram of a CDI cell as used in this study. (b) Operational cycles where discharge voltage is varied $\left(V_{\text {disch }}=0 \mathrm{~V}\right.$ and $\left.0.3 \mathrm{~V}\right)$ as a function of time.

efficiency below unity. For salt concentrations below $30 \mathrm{mM}$, implementation of ion-exchange membranes increases the charge efficiency $\Lambda$ (see Fig. 5D in Ref. [71]) to values close to one, because co-ions are blocked from leaving the electrode region, and only counterions are transported from spacer to electrode to compensate the electronic charge. The disadvantage of membrane-CDI is the more expensive cell design. Though not well supported by experimental data, theoretical calculations suggest that another route to reach a higher charge efficiency, is to increase the charging voltage to values beyond $1.2 \mathrm{~V}$ [49]. However, because of water splitting, voltages beyond $1.23 \mathrm{~V}$ lead to an increase in leakage currents, that is, a current which results in electrochemical reactions, which is not used to charge the porous carbon electrodes. Therefore, voltages beyond $1.23 \mathrm{~V}$ may result in a higher energy consumption. Also the use of (chemically modified) asymmetric electrodes, and reference electrodes, has been suggested as methods to increase $\Lambda$ [88].

To increase charge efficiency, another route is the use of an increased discharge voltage and, as a consequence, a smaller voltage window for CDI as has been suggested and tested before [125]. This is the route we will reanalyze and extend in the present Chapter by using a fixed value of the voltage during charging, $V_{\mathrm{ch}}=1.2 \mathrm{~V}$, just below the water-splitting voltage, and increase the discharge voltage, $V_{\text {disch }}$, to values above zero, see Fig. $2.1 \mathrm{~B}$. As we will demonstrate, both experimentally and theoretically, increasing $V_{\text {disch }}$ only moderately, to around 
$V_{\text {disch }}=0.3 \mathrm{~V}$, leads to increased values of $\Lambda$, while at the same time the salt adsorption capacity and salt adsorption rate are not affected much (order of 5\%). Because of the reduced voltage window used in our study, the total charge transfer in one cycle decreases significantly, and thus the overall energy consumption is lower. For instance, in our setup, and for $c_{\mathrm{salt}}=20 \mathrm{mM}$ and $V_{\mathrm{ch}}=1.2 \mathrm{~V}$, the energy requirement per ion removed reduces from $\sim 30 \mathrm{kT}$ per ion when $V_{\mathrm{disch}}=0 \mathrm{~V}$, to $\sim 26 \mathrm{kT}$ per ion when $V_{\text {disch }}=0.3 \mathrm{~V}$. The results presented in this Chapter go beyond the pioneering work reported in Ref.[125] where the charging voltage was $V_{\mathrm{ch}}=0.6 \mathrm{~V}$ and therefore charge efficiency $\Lambda$ still stayed far below unity, increasing from $\Lambda=0.4$ to $\Lambda=0.7$ upon increasing the discharge voltage from $V_{\text {disch }}=0 \mathrm{~V}$ to $0.4 \mathrm{~V}$. Another difference is that in Refs.[125, 126] it was argued that increasing $V_{\text {disch }}$ is disadvantageous because of a significant decrease in salt removal per cycle. However, as we will show, when $V_{\text {ch }}$ is the value that we use, $V_{\text {ch }}=1.2 \mathrm{~V}$, then in the relevant range of values of $V_{\text {disch }}$ (up to $0.3 \mathrm{~V}$ ), this decrease is minor.

In addition, we demonstrate that theoretical equilibrium calculations using a recently proposed improved modified Donnan (i-mD) model [49] closely fit the data for charge transfer and salt adsorption, across a range of values of cell voltage and salt concentration, while a porous electrode transport theory for CDI well illustrates dynamic data, i.e., ion transport rates in CDI. We also present experimental and theoretical results for constant-current charging in CDI [127], demonstrating that with an increased discharge voltage the transition period required to reach a stable effluent salinity can be drastically reduced. In summary, our results demonstrate the possibility to increase the charge efficiency in a CDI cycle to values close to unity, leading to a lowered energy consumption, and unchanged salt adsorption, by raising the discharge voltage in CDI to values higher than zero.

\subsection{Theory}

\subsubsection{Equilibrium EDL model}

To rationalize the origin of improved charge efficiency in a CDI cycle, we make use of an electrical double layer (EDL) model for ion adsorption in microporous carbons [124]. Here we will use the improved modified Donnan (i-mD) model [49] which is an EDL-model that assumes full overlap of the diffuse layers in the small micropores $[11,49]$. The i-mD model does not consider ion size effects, but does include a Stern layer capacitance and a non-classical "att"-term that describes how uncharged carbon micropores also adsorb salt. The EDL model describes the ion concentrations inside the intraparticle pore space, or micropores, ("mi"), where we model the formation of the EDL and the adsorption of salt, 


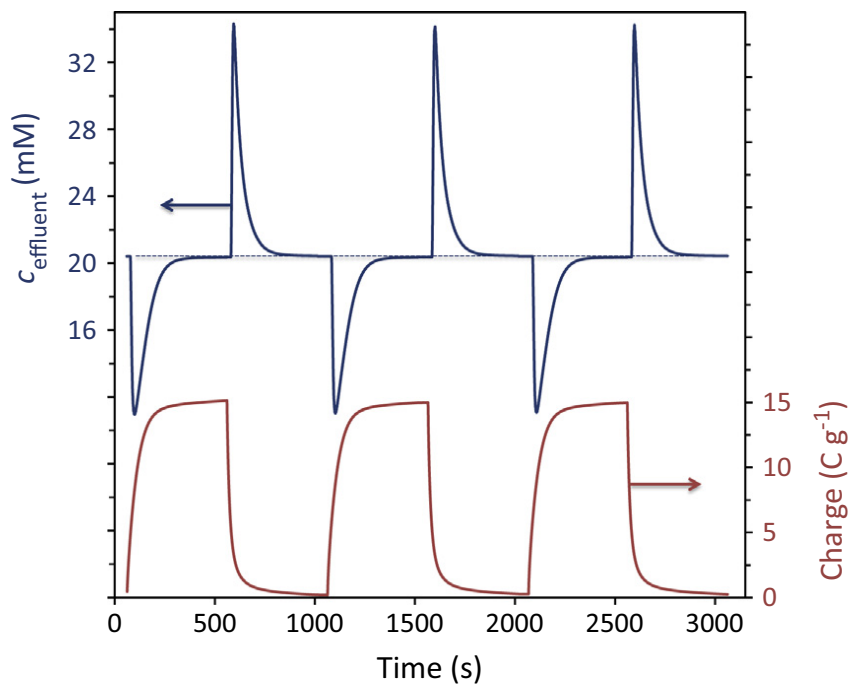

Fig. 2.2: Experimental data of CDI cycles for effluent salt concentration and charge, versus time $\left(V_{\mathrm{ch}}=1.2 \mathrm{~V}, V_{\text {disch }}=0.3 \mathrm{~V}, c_{\infty}=20 \mathrm{mM}\right.$, cycle time $2400 \mathrm{~s}$, only first $500 \mathrm{~s}$ of each charging and discharge step are shown)

as function of the concentration outside the carbon particles (interparticle pore space, or macropores, "mA") [31], which we model as transport pathways for ions to move from the external solution outside the electrode to the micropores. At equilibrium, there is no transport across the electrode, and this macropore concentration is equal to that of the external solution outside the electrode, which we will describe using the subscript " $\infty$ ".

For a 1:1 salt such as $\mathrm{NaCl}$, in the $\mathrm{i}-\mathrm{mD}$ model the micropore ion concentration relates to that outside the pores according to a modified Boltzmann equilibrium,

$$
c_{\mathrm{mi}, i}=c_{\infty} \cdot \exp \left(-z_{i} \cdot \Delta \phi_{\mathrm{D}}+\mu_{\mathrm{att}}\right)
$$

where $c_{\infty}$ is the outside salt concentration, $z_{i}$ is the valency of the ion, and $\Delta \phi_{\mathrm{D}}$ the Donnan potential, i.e., the potential increase when going from outside to inside the carbon particle. This is a dimensionless number and can be multiplied by the thermal voltage $V_{\mathrm{T}}=R T / F(\sim 25.7 \mathrm{mV})$ to obtain the Donnan voltage with dimension $\mathrm{V}$. The non-Boltzmann energy term $\mu_{\text {att }}$ is dimensionless and can be multiplied by $R T$ to obtain a molar energy with dimension $\mathrm{J} / \mathrm{mol}$. We will assume equal values of $\mu_{\text {att }}$ for anion and cation [49]. Inside the carbon micropores, the micropore ionic charge density (per unit micropore volume, dimension $\mathrm{mol} / \mathrm{m}^{3}=\mathrm{mM}$ ) is given by

$$
\sigma_{\mathrm{mi}}=\sum_{i} z_{i} \cdot c_{\mathrm{mi}, i} .
$$


This ionic charge $\sigma_{\mathrm{mi}}$ is compensated by the electronic charge in the carbon matrix: $\sigma_{\mathrm{mi}}+\sigma_{\text {elec }}=0$. When using this simple equation we exclude chemical surface charge effects, but such effects can be included [41, 128]. Next, the ionic charge density relates to the Stern potential, $\Delta \phi_{\mathrm{S}}$, according to

$$
\sigma_{\mathrm{mi}}=-C_{\mathrm{S}} \cdot \Delta \phi_{\mathrm{S}} \cdot V_{\mathrm{T}} / F
$$

where $C_{\mathrm{S}}$ is the Stern layer capacitance in $\mathrm{F} / \mathrm{m}^{3}$. For $C_{\mathrm{S}}$ we use the expression

$$
C_{\mathrm{S}}=C_{\mathrm{S}, 0}+\alpha \cdot \sigma_{\mathrm{mi}}^{2} .
$$

From this point onward we assume symmetry and only describe an experiment with two equal electrodes (equal mass and composition) [129]. We describe in this section salt adsorption and charge in a CDI-cycle operating at two discrete values of the cell voltage $V_{\text {cell }}$, namely $V_{\text {ch }}$ and $V_{\text {disch }}$ (for "charge" and "discharge"), where at both levels equilibrium is established.

Combination of Eqs. (2.1) and (2.2) leads to

$$
\sigma_{\mathrm{mi}}=c_{\text {cation,mi }}-c_{\text {anion,mi }}=-2 \cdot c_{\infty} \cdot \exp \left(\mu_{\text {att }}\right) \cdot \sinh \left(\Delta \phi_{\mathrm{D}}\right)
$$

and

$$
c_{\text {ions }, \mathrm{mi}}=c_{\text {cation,mi }}+c_{\text {anion }, \mathrm{mi}}=2 \cdot c_{\infty} \cdot \exp \left(\mu_{\text {att }}\right) \cdot \cosh \left(\Delta \phi_{\mathrm{D}}\right) .
$$

The above equations must be supplemented with

$$
V_{\text {cell }} / V_{\mathrm{T}}=2 \cdot\left|\Delta \phi_{\mathrm{D}}+\Delta \phi_{\mathrm{S}}\right|
$$

where $V_{\text {cell }}$ is either $V_{\text {ch }}$ or $V_{\text {disch }}$. In the i-mD model [49], in contrast to the previous mD-model $[11,11,35,40,41,47,80,128,130]$, the energy term $\mu_{\text {att }}$ is not taken as a constant, but is given by the ratio of an energy density $E$ divided by the total micropore ions concentration, $c_{\text {ions, mi }}$,

$$
\mu_{\text {att }}=E / c_{\text {ions,mi }} \text {. }
$$

This modification, used in the i-mD model, has a significant effect in improving the precision of the mD-model, especially to describe the influence of salinity (see Fig. 4 in Ref. [49]) without introducing an extra "fit" parameter. As Ref. [49] demonstrated, the use of Eq. (2.8) gives an excellent description of equilibrium data in CDI, both for salt adsorption and charge, as function of both external salt concentration and of cell voltage.

To calculate the charge $\Sigma_{\mathrm{F}}$ that is transferred from one electrode to the other during charging (denoted by superscript "ch"), and back during discharge ("disch"), we multiply the micropore charge density $\sigma_{\mathrm{mi}}$ in one electrode, by the volume of 
micropores per gram of electrode, $v_{\mathrm{mi}}$, and by Faraday's number $F$, do this for charging and discharge, and take the magnitude of the difference

$$
\Sigma_{\mathrm{F}}=\frac{1}{2} \cdot F \cdot v_{\mathrm{mi}} \cdot\left|\sigma_{\mathrm{mi}}^{\mathrm{ch}}-\sigma_{\mathrm{mi}}^{\mathrm{disch}}\right|
$$

where $\Sigma_{\mathrm{F}}$ has dimension $\mathrm{C} / \mathrm{g}$. For the salt adsorption of a cell pair we have

$$
\Gamma_{\mathrm{m}, \mathrm{salt}}=\frac{1}{2} \cdot M_{\mathrm{w}, \mathrm{NaCl}} \cdot v_{\mathrm{mi}} \cdot\left(c_{\mathrm{ions}, \mathrm{mi}}^{\mathrm{ch}}-c_{\mathrm{ions}, \mathrm{mi}}^{\mathrm{disch}}\right)
$$

which has dimension $\mathrm{g} / \mathrm{g}\left(\mathrm{M}_{\mathrm{w}, \mathrm{NaCl}}=58.44 \mathrm{~g} / \mathrm{mol}\right)$. In Eqs. (2.9) and (2.10) the factor $\frac{1}{2}$ stems from the convention in CDI to define salt adsorption and charge by the mass of anode and cathode together.

The charge efficiency $\Lambda$ is the ratio of salt adsorption over charge transfer in a CDI cycle, and is given by

$$
\Lambda=\frac{F}{\mathrm{M}_{\mathrm{w}, \mathrm{NaCl}}} \cdot \frac{\Gamma_{\mathrm{m}, \mathrm{salt}}}{\Sigma_{\mathrm{F}}} .
$$

In case $c_{\infty}^{\text {ch }}=c_{\infty}^{\text {disch }}$ (the external salt concentration is the same before and after charging, as in the Single Pass-method where the inflow salt concentration is always the same), and we discharge at zero voltage, which in our model implies $\sigma_{\mathrm{mi}}^{\text {disch }}=0$ and thus $\Delta \phi_{\mathrm{D}}^{\text {disch }}=0$, Eq. (2.11), with the substitution of Eqs. (2.5), (2.6), (2.9) and (2.10), results in [11, 47, 49, 128]

$$
\Lambda=\frac{c_{\mathrm{ions}, \mathrm{mi}}^{\mathrm{ch}}-c_{\mathrm{ions}, \mathrm{mi}}^{\mathrm{disch}}}{\left|\sigma_{\mathrm{mi}}^{\mathrm{ch}}\right|}=\frac{\cosh \left(\Delta \phi_{\mathrm{D}}^{\mathrm{ch}}\right)-\exp \left(\mu_{\mathrm{att}}^{\mathrm{disch}}-\mu_{\mathrm{att}}^{\mathrm{ch}}\right)}{\sinh \left(\left|\Delta \phi_{\mathrm{D}}^{\mathrm{ch}}\right|\right)}
$$

which clearly shows the general feature that the theoretically predicted value for the charge efficiency directly depends on the diffuse layer (Donnan) voltage during charging, $\Delta \phi_{\mathrm{D}}^{\mathrm{ch}}$, and not on parameters related to the Stern layer. Note that in the i-mD model, where $\mu_{\text {att }}$ depends on $c_{\text {ions,mi }}$ (which in turn depends on $\left.\Delta \phi_{\mathrm{D}}^{\mathrm{ch}}\right)$, charge efficiency is no longer given by $\Lambda=\tanh \left(\left|\Delta \phi_{\mathrm{D}}^{\mathrm{ch}}\right| / 2\right)$ as it is in the mD-model which uses a fixed value of $\mu_{\text {att }}[40,42]$.

\subsubsection{CDI porous electrode transport theory}

To describe the dynamics of salt electrosorption and charge in porous carbon film electrodes forming a CDI cell, we jointly consider ion transport through the space between the carbon particles, that is, through the large transport pathways across the electrode (interparticle pore volume), which we call the macropores (subscript: $\mathrm{mA}$ ), and the electrosorption of ions inside carbon particles (intraparticle pore volume) which we call the micropores (subscript: mi) [41, 131, 132]. This classical model considers a perfect bimodal pore size distribution, of one type 
of macropore and one type of micropore, and neglects mesopores [56, 133]. In the present work these mesopores are introduced in Eq. (2.14). This mesoporosity describes a charge-neutral volume inside carbon particles, having the same salt concentration as the nearby macropores, but without sustaining ion transport. The CDI porous electrode transport theory describes ion electromigration through the spacer and electrode macropores, and the subsequent storage of ions and electronic charge in the EDLs in the micropores [42]. The geometry considered is based on two porous electrodes placed parallel, with a flat planar slit, called the transport channel or spacer, placed in between. In the direction of flow, this transport channel is mathematically divided into $M$ sequential subcells, see Fig. 2 in Ref. [35] and Fig. 2 in Ref. [42]. In the following section, we first describe a single sub-cell and the ion transport into the electrode. Next we describe how all sub-cells can be combined in a unified model for the full CDI system. We focus on a monovalent salt solution, assuming that the two ion diffusion coefficients are equal (as for $\mathrm{KCl}$ ). We neglect tortuosity effects.

In the CDI porous electrode transport model, two coupled one-dimensional partial differential equations must be solved along with additional algebraic equations to evaluate the transport and adsorption of salt across the porous carbon electrodes. The model describes, as a function of the depth in the electrode $x$, and time $t$, four coupled variables: (1) the salt concentration in the macropores, $c_{\mathrm{mA}} ;(2)$ the electrostatic potential there, $\phi_{\mathrm{mA}} ;(3)$ the charge density in the micropores, $\sigma_{\mathrm{mi}}$; and (4) the ions concentration in the micropores, $c_{\mathrm{ions}, \mathrm{mi}}$.

The spacer channel between the two electrodes is described as a series of subcells with a salt concentration $c_{\mathrm{sp}}$ that is only a function of time, described by the salt mass balance

$$
p_{\mathrm{sp}} V_{\text {sub-cell }} \frac{\partial c_{\mathrm{sp}}}{\partial t}=-J_{\text {ions }} A+\Phi_{\mathrm{v}}\left(c_{\mathrm{sp}, \text { in }}-c_{\mathrm{sp}}\right)
$$

where $V_{\text {sub-cell }}\left(\right.$ in $\mathrm{m}^{3}$ ) is the geometrical volume of the sub-cell, $p_{\mathrm{sp}}$ is the open porosity of the spacer channel, $A$ the exchange area of one sub-cell with one electrode (in $\mathrm{m}^{2}$ ), and $\Phi_{\mathrm{v}}$ the volumetric flow rate of water running through the cell, i.e., along the electrodes (in $\mathrm{m}^{3} / \mathrm{s}$ ). We assume the two electrodes to behave symmetrically and as a consequence the ion flux into one electrode, $J_{\text {ions }}$, is equal to the salt flux into both electrodes.

Based on the Nernst-Planck equation [134], transport within the porous electrode is described by two coupled partial differential equations. First, a differential salt mass balance can be set up, given by

$$
\frac{\partial}{\partial t}\left(\left(p_{\mathrm{mA}}+p_{\text {meso }}\right) c_{\mathrm{mA}}+\frac{1}{2} p_{\mathrm{mi}} c_{\text {ions }, \mathrm{mi}}\right)=p_{\mathrm{mA}} D \frac{\partial^{2} c_{\mathrm{mA}}}{\partial x^{2}}
$$

with the electrode position $x$ between $0<x<L_{\text {elec }}$, where $L_{\text {elec }}$ is the electrode thickness, and where $D$ is the average ion diffusion coefficient in the macropores, 
and where $p_{\mathrm{mA}}, p_{\text {meso }}$, and $p_{\mathrm{mi}}$ are the macro-, meso-, and microporosity in the electrode.

As Eq. (2.14) shows, we consider all fluxes to be in only one direction, namely the direction into the electrode, i.e., at cross-angles with the general flow direction of the solution through the channel, see Fig. 2 in Ref. [42].

The second partial differential equation describes the charge in the micropores and is given by

$$
p_{\mathrm{mi}} \frac{\partial \sigma_{\mathrm{mi}}}{\partial t}=2 p_{\mathrm{mA}} D \frac{\partial}{\partial x}\left(c_{\mathrm{mA}} \frac{\partial \phi_{\mathrm{mA}}}{\partial x}\right) .
$$

At each position in the electrodes, the macropore potential $\phi_{\mathrm{mA}}$ is related to the potential $\phi_{1}$ in the carbon matrix according to

$$
\phi_{1}-\phi_{\mathrm{mA}}=\Delta \phi_{\mathrm{D}}+\Delta \phi_{\mathrm{S}}
$$

with expressions for $\Delta \phi_{\mathrm{D}}$ and $\Delta \phi_{\mathrm{S}}$ given by Eqs. (2.3)-(2.6). Please note that, in this Thesis, we assume that there is no electronic resistance across the electrode, which is supported by experimental work presented in Chapter 3, Box 3.1. At each position the micropore ions concentration $c_{\text {ions,mi }}$ is related to charge $\sigma_{\mathrm{mi}}$ according to

$$
c_{\mathrm{ions}, \mathrm{mi}}^{2}=\sigma_{\mathrm{mi}}^{2}+\left(2 \cdot c_{\mathrm{mA}} \cdot \exp \left(\mu_{\mathrm{att}}\right)\right)^{2} .
$$

With the potential in the carbon matrix set to zero $\left(\phi_{1}=0\right)$, the cell voltage is given by

$$
V_{\text {cell }}=2 V_{\mathrm{T}}\left(\left.\phi_{\mathrm{mA}}\right|_{x=0}+\Delta \phi_{\mathrm{sp}}\right)
$$

where " $x=0$ " refers to the front side of the electrode (in contact with the spacer channel), and where $\Delta \phi_{\mathrm{sp}}$ is the voltage drop across half the spacer channel, obtained from

$$
J=-4 c_{\mathrm{sp}} p_{\mathrm{sp}} D L_{\mathrm{sp}}^{-1} \Delta \phi_{\mathrm{sp}}
$$

where $J$ is the current density (in $\mathrm{mol} / \mathrm{m}^{2} / \mathrm{s}$ ). The ions flux, $J_{\text {ions }}$, out of the spacer channel required in Eq. (2.13) is equal to the ions flux directed into the electrode

$$
J_{\text {ions }}=-\left.2 p_{\mathrm{mA}} D \frac{\partial c_{\mathrm{mA}}}{\partial x}\right|_{x=0}
$$

and a similar relation holds for the current density,

$$
J=-\left.2 p_{\mathrm{mA}} D\left(c_{\mathrm{mA}} \frac{\partial \phi_{\mathrm{mA}}}{\partial x}\right)\right|_{x=0} .
$$

For an overview of the required initial and boundary conditions in $c_{\mathrm{mA}}, \phi_{\mathrm{mA}}$, $\sigma_{\mathrm{mi}}$ and $c_{\mathrm{ions}, \mathrm{mi}}$ in each sub-cell, see Suppl. Inf. in Ref. [42]. In the full model for the CDI-cell, $M$ of the sub-cells as described above, are placed sequentially in the direction of flow. In this direction salt is transported through the spacer channel 
downstream, as described by Eq. (2.13) with $c_{\mathrm{sp}}$ the concentration in sub-cell $i$ and $c_{\mathrm{sp} \text {,in }}$ the concentration in the up-stream sub-cell $i-1$. The concentration in the last sub-cell $(i=M)$ is equal to the effluent concentration. The sub-cell volume is equal to the total spacer channel volume (height times electrode area) divided by the number of sub-cells, $M$. In the present work we do not include a "dead volume" after the stack as in Refs. [42,46]. The current density per cell, $I$ (in $\mathrm{A} / \mathrm{m}^{2}$ ) is calculated from $I=F / M \cdot \sum_{i} J_{i}$, where $i$ is a summation over all sub-cells. This current density, $I$, can be integrated over time and multiplied by the cell cross-sectional area to obtain the total stored charge. The charge stored in an electrode can also be calculated by averaging the local charge density $\sigma_{\mathrm{mi}}$ (Eq. (2.15)) and multiplying by Faraday's number, the microporosity $p_{\text {mi }}$, and the volume of the electrode. Dividing by electrode mass (anode and cathode together) gives us the charge $\Sigma_{\mathrm{F}}(\mathrm{C} / \mathrm{g})$ as plotted in Figs. 2.2-2.4.

\subsection{Materials and methods}

\subsubsection{Electrode preparation}

Carbon composite electrode was fabricated using YP-50F (Kuraray, Japan), carbon black (Vulcan XC72R, Cabot Corp., Boston, MA), and a binder (85:5:10 in weight ratio). In order to prepare the binder solution (3 wt\%), polyvinylidene fluoride (PVDF, Kynar HSV 900, Arkema Inc., Philadelphia, PA) was dissolved in N-methyl-2-pyrrolidone (NMP) and vigorously stirred for $24 \mathrm{~h}$ to secure homogeneity. After YP-50F and carbon black were mixed with the prepared binder solution, it was intensively blended in a ball-milling machine for $40 \mathrm{~min}$ (500 $\mathrm{rpm}$ ) and the resulting carbon slurry was used for casting. The electrode casting was carried out on a glass plate with a doctor blade (thickness $500 \mu \mathrm{m}$ ). Afterwards, the electrode was directly transferred into deionized water together with the glass plate to solidify the binder. The resulting carbon electrode $(\sim 260 \mu \mathrm{m})$ was cut into square pieces $\left(6 \times 6 \mathrm{~cm}^{2}\right)$ with a small square $\left(1.5 \times 1.5 \mathrm{~cm}^{2}\right)$ located at the center.

\subsubsection{CDI experiments}

Fig. 2.1A shows a schematic diagram of the CDI system used in this study. Each cell consists of a pair of graphite current collectors, a pair of carbon electrodes, and a spacer (AP20, Glass Fiber Filter, Millipore, MA, uncompressed thickness $\sim 380 \mu \mathrm{m})$. A stack was constructed of $N_{\mathrm{s}}=4$ of such cells and installed in a rectangular Teflon housing which was sealed off. As depicted in Fig. 2.1A, water is pumped from outside to inside through the spacer using a peristaltic pump 
(flow rate $30 \mathrm{~mL} / \mathrm{min}$ for the stack of 4 cells), and the outlet conductivity and $\mathrm{pH}$ were continuously monitored. The experiments were conducted in $\mathrm{NaCl}$ solutions of different concentration $\left(c_{\infty}=5,20,80 \mathrm{mM}\right)$ prepared in a vessel (10 L) with $\mathrm{N}_{2}$ purging. While the solution flows through the CDI stack, the system was controlled by a power supply (IviumStat, Ivium Technologies, the Netherlands). The following voltage differences across each cell were imposed: a voltage $V_{\text {ch }}$ during charging (deionization, salt adsorption) and $V_{\text {disch }}$ during discharge (regeneration, desorption). For the equilibrium experiments (Figs. 2.3 and 2.4), the duration of each cycle, including the charging and discharge step, which we define as cycle time, $\mathrm{CT}$, was $40 \mathrm{~min}$ while $\mathrm{CT}$ was lower in the experiments of Fig. 2.5 (for each experiment, the discharge time was equal to the charging time). Each experiment was carried out at a distinct value of $V_{\text {disch }}$, ranging from 0 to $0.9 \mathrm{~V}$, while the charging voltage $V_{\text {ch }}$ was always set to $1.2 \mathrm{~V}$ as described in Fig. 2.1B (except for experiments reported in Figs. 2.2 and 2.6).

From the measured electrical current, the charge transferred (back and forth) in one cycle is calculated, while from the measured conductivity (corrected via the measured $\mathrm{pH}$ for the partial conductivity of protons and hydroxyl ions) and from the water flow rate, the salt adsorption is calculated. Note that salt adsorption in $\mathrm{mg} / \mathrm{g}$ of $\mathrm{NaCl}$ is based on the mass of all electrodes in the system (both anode and cathode). The charge was obtained from the charging step (from integrating the current vs. time-plot), after subtracting a small leakage current, which is the current which still flows when equilibrium is reached after $20 \mathrm{~min}$. Note that when the leakage current would not be accounted for, the calculated charge is a few percent higher, and thus values of the charge efficiency are a few percent less than reported. None of the reported results are from the first or second cycle after a new experiment, as the dynamic equilibrium is not yet established: salt adsorption and charge during charging are not the same as the values during discharge. This condition of salt balance and charge balance is well achieved in subsequent cycles.

To calculate the salt adsorption per cycle, as required in Fig. 2.5, we use the data analysis approach discussed in Ref. [27], where the salt adsorption is based on the entire time period that the effluent salt concentration is below the inflow concentration (period from A to B in Fig. 3 in Ref. [27]), a period which is slightly delayed relative to the period of cell charging. Instead, in prior work discussing short cycle times $[35,71,130]$ salt adsorption (desorption) was calculated based on the period of cell charging (discharging). 
Table 2.1: System and electrode dimensions, operational parameters, and settings for theoretical calculations.

\begin{tabular}{|l|l|rl|}
\hline \multicolumn{3}{|c|}{ Experimental parameters } \\
\hline$N_{\mathrm{s}}$ & Number of cells in the CDI stack & 4 & \\
$\rho_{\mathrm{el}}$ & Electrode mass density & 0.40 & $\mathrm{~g} / \mathrm{mL}$ \\
$A_{\text {cell }}$ & Electrode geometric surface area & 33.8 & $\mathrm{~cm}^{2}$ \\
$L_{\mathrm{sp}}$ & Spacer channel thickness (when compressed) & 250 & $\mu \mathrm{m}$ \\
$p_{\mathrm{sp}}$ & Spacer channel porosity (when compressed) & 0.50 & \\
$\Phi_{\mathrm{v}}$ & Flowrate through the CDI stack & 30 & $\mathrm{~mL} / \mathrm{min}$ \\
$T$ & Temperature & 298 & $\mathrm{~K}$ \\
$c_{\infty}$ & Inlet NaCl salt concentration & $5,20,80$ & $\mathrm{mM}$ \\
\hline \multicolumn{3}{|c|}{ Values for use in EDL and transport theory } \\
\hline$v_{\mathrm{mi}}$ & Micropore volume & 0.40 & $\mathrm{~mL} / \mathrm{g}$ \\
$p_{\mathrm{mi}}$ & Microporosity & 0.160 & \\
$p_{\mathrm{meso}}$ & Mesoporosity & 0.068 & \\
$p_{\mathrm{mA}}$ & Macroporosity & 0.566 & \\
$D$ & Average diffusion coefficient of Na ${ }^{+}$ & 1.68 & $10^{-9} \mathrm{~m}^{2} / \mathrm{s}$ \\
& and Cl ${ }^{-}$(in free solution) & 170 & $\mathrm{~F} / \mathrm{mL}^{-1}$ \\
$C_{\mathrm{S}, 0}$ & Stern capacitance in the & & \\
& zero-charge limit & 20 & $\mathrm{~F} \mathrm{~m} \mathrm{~mol}^{-2}$ \\
$\alpha$ & Charge dependence of Stern capacitance & 220 & $\mathrm{kT} \mathrm{mol} \mathrm{m}{ }^{-3}$ \\
$E$ & Micropore ion-correlation energy & 1 & \\
$M$ & Number of mathematical sub-cells &
\end{tabular}

\subsubsection{Data analysis to derive EDL-properties}

To fit the i-mD model (EDL theory) to the data, four parameters must be adjusted as summarized in Table 2.1: $v_{\mathrm{mi}}, E, C_{\mathrm{S}, 0}$ and $\alpha$. The analysis is based on the following procedures. First of all, we note that predictions of the theory for $\Lambda$, or $\Gamma_{\mathrm{m} \text {,salt }}$, as function of $\Sigma_{\mathrm{F}}$ (and vice-versa) depend on the micropore volume $v_{\mathrm{mi}}$ and on the energy $E$, but are independent of the Stern layer parameters $C_{\mathrm{S}, 0}$ and $\alpha$. Thus, we first plot data for $\Gamma_{\mathrm{m} \text {,salt }}$ versus $\Sigma_{\mathrm{F}}$ (not shown, but similar to Fig. 3G in Ref. [49]) and compare with the i-mD model to find appropriate values for $E$ and $v_{\mathrm{mi}}$. A second comparison considers the interesting fact that the Donnan potential, $\Delta \phi_{\mathrm{D}}$, uniquely relates $c_{\mathrm{ions} \text {,mi }}$ and $\sigma_{\mathrm{mi}}$ to one another, see Eqs. (2.5) and (2.6) (with the energy $E$ as parameter), all independent of the value of $v_{\mathrm{mi}}$. Thus, together with the direct comparisons of $\Gamma_{\mathrm{m} \text {,salt }}$ and $\Sigma_{\mathrm{F}}$ vs $V_{\text {cell }}$, we have various parallel methods for reliable data fitting. Figs. 2.3 and 2.4 demonstrate that, across a large dataset (varying $c_{\infty}, V_{\mathrm{ch}}$ and $V_{\text {disch }}$ ), we can very satisfactorily fit all data of $\Gamma_{\mathrm{m} \text {,salt }}$ and $\Sigma_{\mathrm{F}}$ using the $\mathrm{i}-\mathrm{mD}$ model. The EDL 
parameters we obtain are close to those previously derived in Refs. [35, 49] where a commercial activated carbon-based electrode was considered.

An important point to note is that the micropore volume of $v_{\mathrm{mi}}=0.400 \mathrm{~mL} / \mathrm{g}$ (per gram electrode) that we use in the theory is less than the total pore volume measured by gas sorption analysis, $v_{G S A}$, which for Kuraray YP-50 is about 0.67 $\mathrm{mL}$ per gram of activated carbon $(0.57 \mathrm{~mL} / \mathrm{g}$ electrode), see Refs. [42, 76]. To get an optimum fit of the $\mathrm{i}-\mathrm{mD}$ model to the data, $v_{\mathrm{mi}}$ is best used as a fitting parameter, not necessarily the same as the total pore volume inside the carbon particles. Thus, we must consider that the fitted value of $v_{\mathrm{mi}}$ is different from $v_{G S A}$, and we suggest to call this difference $(0.57-0.40 \mathrm{~mL} / \mathrm{g}$ in the present case) mesoporosity, $v_{\text {meso }}$.

In the mesopores, like in the nearby macropores, we assume equal concentrations of cations and anions (for a 1:1 salt), at the same concentration as in the nearby macropores. The only difference with the macropores is that the ions in the mesopores do not contribute to ion transport across the electrode. The macropore volume, $p_{\mathrm{mA}}$, represents the space located in between the carbon particles and follows from the relation $p_{\mathrm{mi}}+p_{\text {meso }}+p_{\mathrm{sk}}+p_{\mathrm{mA}}=1$, where $p_{\mathrm{mi}}$ is given by $p_{\mathrm{mi}}=v_{\mathrm{mi}} \cdot \rho_{\text {elec }}$ and $p_{\text {meso }}$ by $p_{\text {meso }}=v_{\text {meso }} \cdot \rho_{\text {elec }}$. Parameter $p_{\text {sk }}$ is the volume fraction of "skeleton" in the electrode, which in turn can be calculated by dividing $\rho_{\text {elec }}$ by $\rho_{\text {sk }}$, the mass density of the skeleton material. The density $\rho_{\mathrm{sk}}=1.93 \mathrm{~g} / \mathrm{mL}$ is obtained from $\rho_{\mathrm{sk}}=m_{\mathrm{c}} \cdot \rho_{\mathrm{c}}+\left(1-m_{\mathrm{c}}\right) \cdot \rho_{\mathrm{b}}$ where $m_{\mathrm{c}}$ is the weight fraction of two carbon constituents together (activated carbon and carbon black), $m_{\mathrm{c}}=0.9, \rho_{\mathrm{c}}$ the mass density of the carbon, $\rho_{\mathrm{c}}=1.95 \mathrm{~g} / \mathrm{mL}$, and $\rho_{\mathrm{b}}$ the density of the binder, $\rho_{\mathrm{b}}=1.78 \mathrm{~g} / \mathrm{mL}[42]$.

\subsection{Results and discussion}

To illustrate the response of a CDI cell to the cell voltage signal as depicted in Fig. 2.1B, we show in Fig. 2.2 the typical CDI behavior of the effluent salt concentration profile over time, and the accumulated charge (current integrated over time), $\Sigma_{\mathrm{F}}$. As can be observed, the effluent concentration drops as the charging starts and in time recovers to the initial value, where the area under the base line (dashed line representing the inlet salt concentration) relates to the amount of salt adsorption, $\Gamma_{\mathrm{m} \text {,salt }}$. Incorporating the factor $F / \mathrm{M}_{\mathrm{w}, \mathrm{NaCl}}$, the ratio of $\Gamma_{\mathrm{m} \text {,salt }}$ over $\Sigma_{\mathrm{F}}$ is the charge efficiency, $\Lambda$, see Eq. (2.11). Experimental data for $\Sigma_{\mathrm{F}}, \Gamma_{\mathrm{m} \text {,salt }}$ and $\Lambda$ are further analyzed in Figs. 2.3-2.6. An important operational parameter is the cycle time, CT, which is the total duration of the charging and discharge step. Note that the charging time is equal to the discharge time. The value of $\mathrm{CT}$ in the experiments reported in Figs. 2.2-2.4 is $40 \mathrm{~min}$, while it is reduced to values as low as $2 \mathrm{~min}$ in Fig. 2.5. Because of the long $\mathrm{CT}$, the data 
reported in Figs. 2.3 and 2.4 can be described by the equilibrium EDL model explained in Section 2.2.1, without considering porous electrode ion transport theory which is used in Figs. 2.5 and 2.6. Note that in a series of experimental CDI cycles as reported in Fig. 2.2, the apparent accumulated charge slowly shifts upward because of a leakage current (see Experimental section). In Fig. 2.2 this shift is removed in data-processing, and each subsequent charging cycle shifted back to zero charge.

From equilibrium cycles such as reported in Fig. 2.2, data on salt adsorption $\Gamma_{\mathrm{m} \text {,salt }}$ and charge $\Sigma_{\mathrm{F}}$ can be extracted which are reported in Figs. 2.3 and 2.4. Fig. 2.3 shows experimental results (points) and theory (lines) of salt adsorption, charge and charge efficiency as function of the salt concentration and the charging voltage, $V_{\mathrm{ch}}$. Fig. 2.3 follows the classical operational method of CDI where always $V_{\text {disch }}=0 \mathrm{~V}$, as for instance in Refs. [35, 42, 47, 71, 80, 112, 128]. Note that here in Fig. 2.3 we present data as function of charging voltage, for a larger range of salinities than before [49], from $c_{\infty}=5$ to $80 \mathrm{mM}$. Fig. 2.3A-C show data and theory for salt adsorption in $\mathrm{mg} / \mathrm{g}$ (left $\mathrm{y}$-axis) and charge in $\mathrm{C} / \mathrm{g}$ (right y-axis), in such a way that for $\Lambda=1$, the two lines (data sets) should overlap. As clearly they do not overlap, the charge efficiency is less than unity, $\Lambda<1$. Fig. 2.3A shows that at the lowest salt concentration of $c_{\infty}=5 \mathrm{mM}$ the two lines (data sets) are closest, and $\Lambda$ is the closest to unity. Fig. 2.3A-C show that as function of $V_{\mathrm{ch}}$, both $\Sigma_{\mathrm{F}}$ and $\Gamma_{\mathrm{m} \text {,salt }}$ increase, but the latter increases faster (relatively) and thus their ratio, which is $\Lambda$, increases with $V_{\text {ch }}$. As function of $c_{\infty}$ (for a given $\left.V_{\mathrm{ch}}\right), \Sigma_{\mathrm{F}}$ increases while $\Gamma_{\mathrm{m} \text {,salt }}$ is constant (in the range of $c_{\infty}$ studied). Thus $\Lambda$ increases with $V_{\text {ch }}$ and decreases with $c_{\infty}$, as shown in more detail in Fig. 2.3D. In the present data range, $\Gamma_{\mathrm{m} \text {,salt }}$ does not vary with $c_{\infty}$, in line with data in Ref. [49] which showed that for lower and higher $c_{\infty}, \Gamma_{\mathrm{m} \text {,salt }}$ decreases again. This non-monotonic trend cannot be explained by a simple adsorption isotherm that describes the adsorption of an uncharged solute, which would always predict $\Gamma_{\mathrm{m} \text {,salt }}$ to increase with $c_{\infty}$. Instead, the observed dependence of $\Gamma_{\mathrm{m} \text {,salt }}$ on $c_{\infty}$ (to first increase, and then to decrease again) is the consequence of the salt storage mechanism in CDI, where salt is stored as individual ions in the electrical double layers of two oppositely charged porous electrodes.

Fig. 2.4 presents results where we fix $V_{\text {ch }}$ at $1.2 \mathrm{~V}$ and vary $V_{\text {disch }}$ for different values of the salt concentration and again plot salt adsorption, charge and charge efficiency. As can be seen, theory again properly describes the data (parameter settings given in Table 2.1). Experimentally we observe that charge efficiency increases with increasing $V_{\text {disch }}$, see Fig. $2.4 \mathrm{D}$, which is also in alignment with theoretical predictions. Charge efficiency increases to values close to unity, especially for $V_{\text {disch }}$ beyond $0.3 \mathrm{~V}$. In Fig. 2.4A-C, salt adsorption and charge are separately displayed, where the ratio of these two parameters refers 

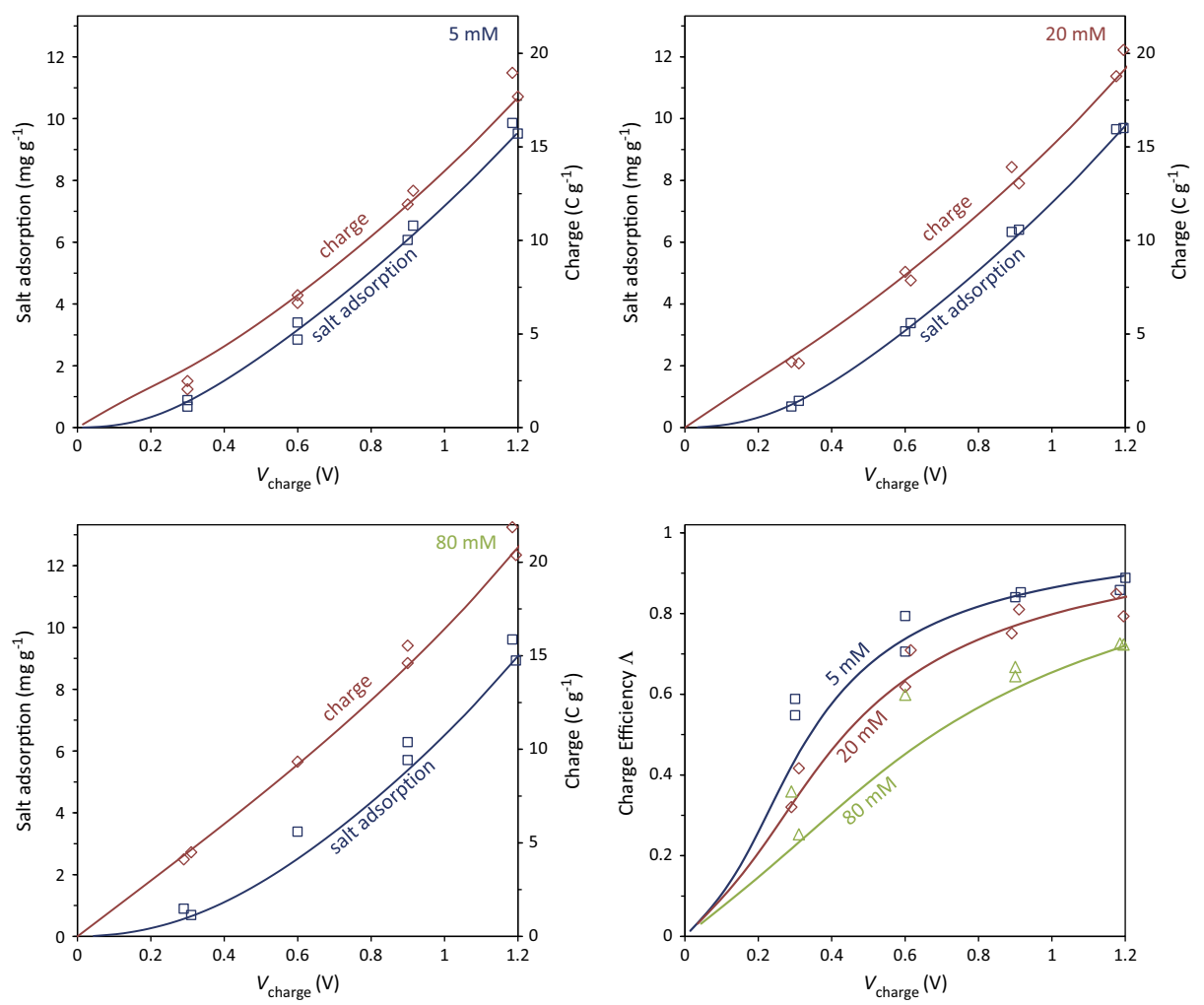

Fig. 2.3: Equilibrium salt adsorption, charge, and charge efficiency as function of salt concentration and $V_{\mathrm{ch}}$, which is the cell voltage during charging $\left(V_{\mathrm{disch}}=0 \mathrm{~V}\right)$. Solid lines obtained according to the i-mD model (parameters in Table 2.1). 
to charge efficiency. As confirmed in Fig. 2.4D, we observe that these two parameters (charge and salt adsorption) get closer as $V_{\text {disch }}$ increases. As expected, the charge decreases quite linearly when $V_{\text {disch }}$ increases (note that the increase in $V_{\text {disch }}$ leads to a decrease in the difference $V_{\mathrm{ch}}-V_{\text {disch }}$ ), indicating the typical capacitive behaviour of carbon electrodes. On the other hand, the dependence of salt adsorption on $V_{\text {disch }}$ is different from that of the charge. In contrast to the dependence of charge on $V_{\text {disch }}$, salt adsorption in a cycle remains quite invariant with $V_{\text {disch }}$, as long as $V_{\text {disch }}$ does not go beyond $0.3 \mathrm{~V}$. This result confirms that charge efficiency can be increased by increasing $V_{\text {disch }}$ to values up and including $V_{\text {disch }}=0.3 \mathrm{~V}$ with only a marginal loss of the salt adsorption per cycle. At $c_{\infty}=20$ $\mathrm{mM}$, upon increasing $V_{\text {disch }}$ from 0 to $0.3 \mathrm{~V}$, charge decreases by $20 \%$ while salt adsorption goes down by only $7 \%$. This implies that charge efficiency goes up by $14 \%$, and thus energy consumption per ion removed goes down by that number of $14 \%$, as will be explained below.

An explanation for the positive effect of increasing $V_{\text {disch }}$ is as follows. When the cell is charged at $V_{\mathrm{ch}}=1.2 \mathrm{~V}$ followed by a discharge at $V_{\mathrm{disch}}=0 \mathrm{~V}$ (following the conventional method), the adsorption of counterions is accompanied by the expulsion of co-ions, so that charge efficiency drops to values below unity [125]. This undesirable behaviour of co-ions can be minimized by increasing the discharge voltage $[125,126]$. Now, with values of $V_{\text {disch }}$ higher than $0 \mathrm{~V}$, a certain amount of co-ions remains expelled during discharge (is not re-adsorbed). In a next cycle, therefore, the expulsion of co-ions is small when charging starts from a value of $V_{\text {disch }}$ higher than $0 \mathrm{~V}$, and thus most of the electronic charge transferred between the two electrodes is utilized solely for the adsorption of counterions.

The increased value of charge efficiency directly implies a reduction in the required energy per ion removed. This can be understood as follows. Because adsorption operates at constant charging voltage, the energy input is directly proportional to the quantity of charge transfer, and thus is inversely proportional to charge efficiency $\Lambda$. For example, for the data at $c_{\infty}=20 \mathrm{mM}$, the energy costs per ion removed is $\sim 30 \mathrm{kT}$ at $V_{\text {disch }}=0 \mathrm{~V}$, and decreases to $\sim 26 \mathrm{kT}$ for $V_{\text {disch }}=0.3 \mathrm{~V}$, a reduction by $14 \%$. In this calculation we have not included possible energy recovery during discharge, which is possible when $V_{\text {disch }}>0 \mathrm{~V}$ [135]. Indeed, for this situation of $V_{\text {disch }}=0.3 \mathrm{~V}$, assuming perfect energy recovery during discharge, the energy per ion removed is reduced to $\sim 20 \mathrm{kT}$, a reduction of about $30 \%$ compared to the original value at $V_{\text {disch }}=0 \mathrm{~V}$. Note that for the separation that was achieved, the thermodynamic minimum energy input (decrease in mixing entropy) is only about $0.2 \mathrm{kT}$ per removed ion, a factor 100 below the actual energy input.

Figs. 2.3 and 2.4 discussed equilibrium EDL-properties. The next task is to analyze the kinetics of the CDI process. To this end we first analyze CDI cycles 

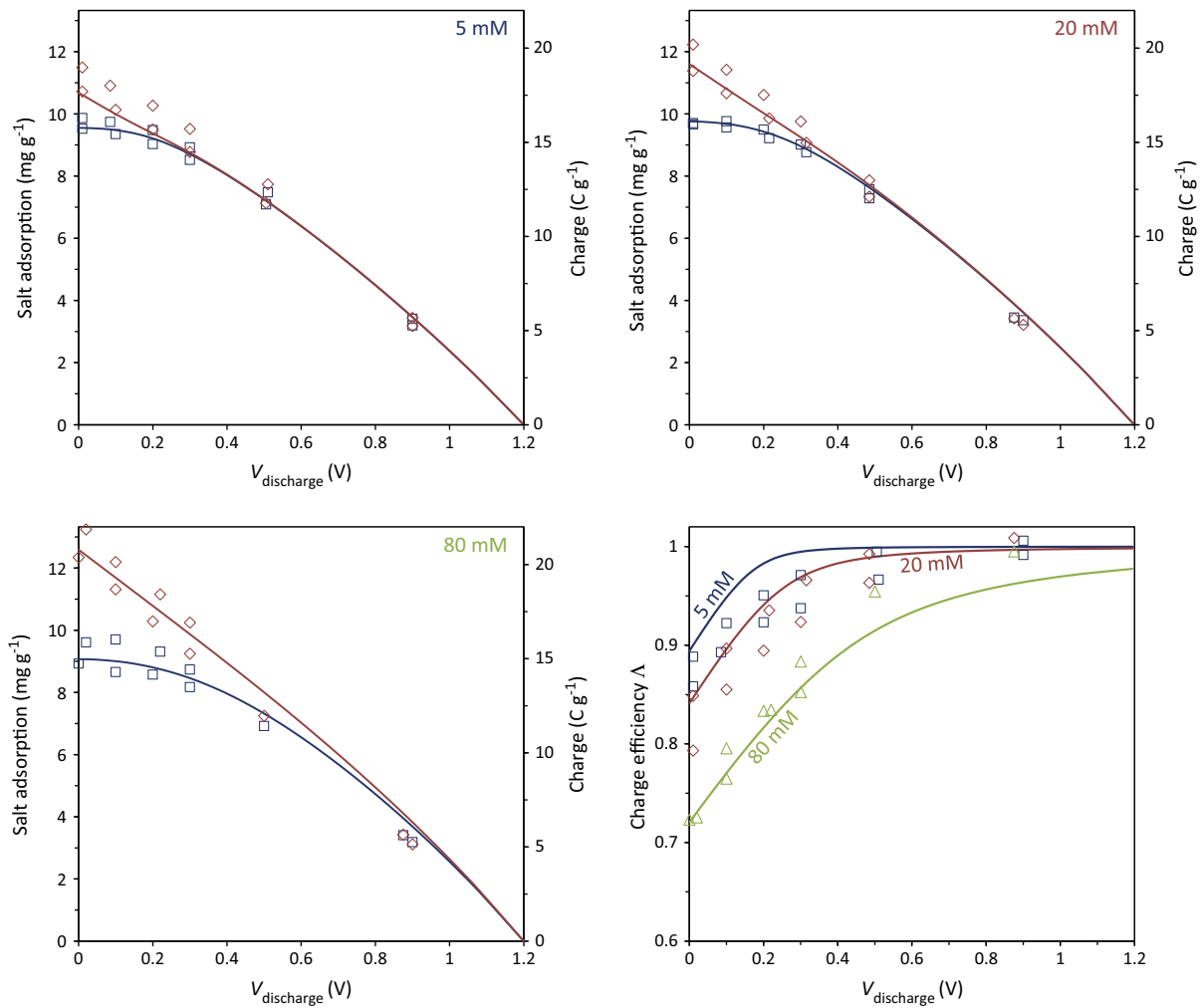

Fig. 2.4: Salt adsorption, charge, and charge efficiency $(\Lambda)$ as function of salt concentration and discharge voltage, $V_{\mathrm{disch}}\left(V_{\mathrm{ch}}=1.2 \mathrm{~V}\right)$. Solid lines obtained according to the i-mD model (parameters in Table 2.1). 

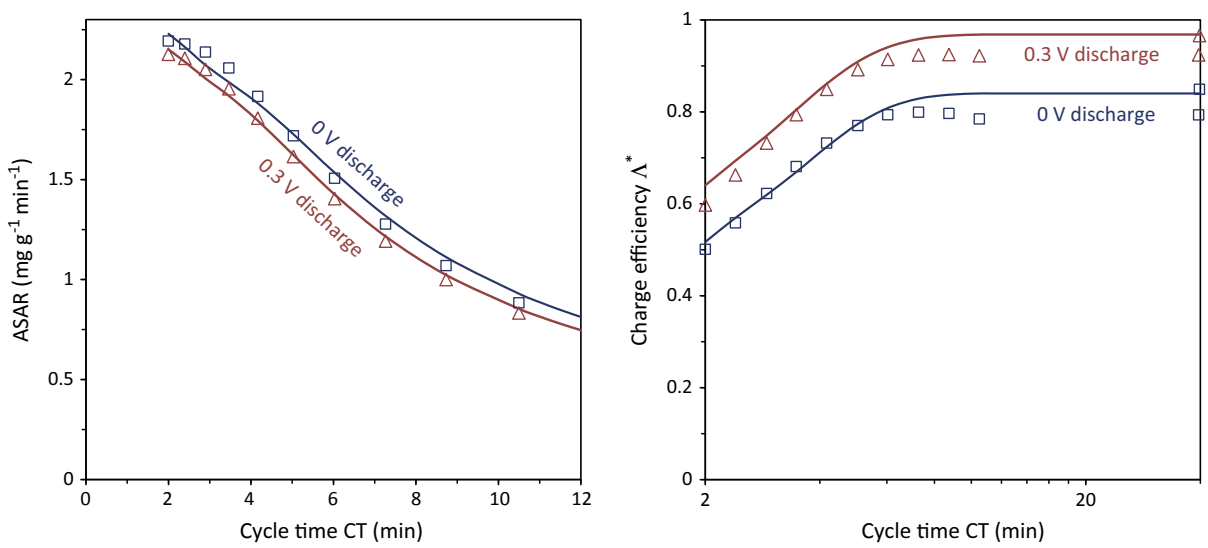

Fig. 2.5: CDI at short cycle times. (a). Average salt adsorption rate as function of cycle time (CT) and as function of $V_{\text {disch }}$. (b). Dynamic charge efficiency $\Lambda^{*}$ as function of CT $\left(V_{\mathrm{ch}}=1.2 \mathrm{~V}, c_{\infty}=20 \mathrm{mM}\right.$, electrode thickness $\left.235 \mu \mathrm{m}\right)$.

at constant voltage (CV), at increasingly short cycle times (CT), see Fig. 2.5. In Fig. 2.5A we plot the average salt adsorption rate, "ASAR" which is the salt adsorption per cycle, divided by CT.

Fig. 2.5A clearly demonstrates, both theoretically and experimentally, that ASAR increases with lowering CT (as also reported in Ref. [35]), and in addition that there is hardly an effect of $V_{\text {disch }}$. Admittedly, we had hoped that at an increased $V_{\text {disch }}$, ASAR will be higher, but this is not the case, and ASAR is actually somewhat less. This prior assumption was based on the idea that with $V_{\text {disch }}=0 \mathrm{~V}$ at the start of each charging or discharge step, co-ions and counterions go in opposite direction through the electrode and into the micropores. This opposite movement would lead to ion-ion friction and thus a retardation in the motion of both types of ions. Increasing $V_{\text {disch }}$ would then lead to a situation where only counterions move in and out of the electrode, undisturbed by other ions moving in the opposite direction. However, results in Fig. 2.5A do not support the hypothesis of a frictional force between ions moving in opposite direction, and apparently this suggested retardation effect is not there, or at least is not strong. The transport model does not include such an effect either, and describes data very well.

Analyzing both the charge transfer $\Sigma_{\mathrm{F}}$ and salt adsorption $\Gamma_{\mathrm{m} \text {,salt }}$ per cycle, Fig. 2.5B shows results of the ratio of the two, the "dynamic" charge efficiency, $\Lambda^{*}$. Here we add the prefix "dynamic" because in contrast to the results of Figs. 2.3 and 2.4, here the charge efficiency is based on a CDI cycle that is not yet at equilibrium. Consequently, theory lines for $\Lambda^{*}$ in Fig. 2.5B are not based on an equilibrium EDL model but are based on the transport model. Both in 
theory and data, Fig. 2.5B shows that shorter cycles result in a lower $\Lambda^{*}$ (vs. the values at longer $\mathrm{CT}$ ) indicating an increase in energy costs per ion removed. This is the case because the required energy per amount of salt removed is inversely proportional to $\Lambda^{*}$ for a fixed charging voltage. As in Fig. 2.4, we observe that operation at $V_{\text {disch }}=0.3 \mathrm{~V}$ gives a higher charge efficiency, and thus the same energetic advantage of operation at increased values of $V_{\text {disch }}$ remains, of about $14 \%$ without energy recovery and $30 \%$ with energy recovery.

Finally, we show results for constant-current (CC) operation during charging in Fig. 2.6. Constant-current operation is advantageous over constant-voltage (CV)

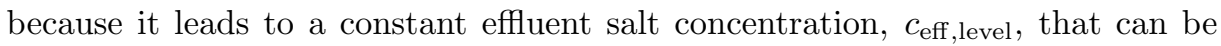
easily tuned by adjusting the current [71]. However, up to now it was argued that this operational mode is only feasible with ion-exchange membranes incorporated in CDI (membrane-CDI), because without membranes (i.e., in normal CDI) it takes a longer time before $c_{\text {eff, level }}$ is reached after switching the current direction, see Fig. 8 in Ref. [11]. In Fig. 2.6 we show our results aiming at reducing the time for $c_{\text {eff, level }}$ to be reached. In these experiments with $\mathrm{CC}$ operation during charging (with a constant current of $0.41 \mathrm{~A}$ applied to the stack of $N_{\mathrm{s}}=4$ cells, thus at a current density of $30.6 \mathrm{~A} / \mathrm{m}^{2}$, until a cell voltage of $V_{\text {cell }}=1.4 \mathrm{~V}$ is reached), the discharge step is always defined by $\mathrm{CV}$ operation at certain values of $V_{\text {disch }}$, always for a duration of $250 \mathrm{~s}$. Fig. $2.6 \mathrm{~A}$ demonstrates that a more rapid drop of salt concentration to $c_{\text {eff, level }}$ is found with increasing $V_{\text {disch }}$, both in the experimental data (points) and in the theoretical calculations (lines). This is due to the fact that at $V_{\text {disch }}=0 \mathrm{~V}$, at the start of a new charging step, co-ions and counterions replace one another, a phenomenon called "ion swapping" in Ref. [136] without resulting desalination. Instead, with increasing $V_{\text {disch }}$ we reach the situation that right after start of a new cycle only counterions are adsorbed (with co-ions remaining outside the micropores at all times).

Fig. 2.6A also shows a theory line for the case that for a short duration of about $20 \mathrm{~s}$ after start of the charging step, we use a twice-higher value of the current. The current vs. time-curve is $I(t)=I_{\text {final }} *(2 \alpha *(1-\tanh (\alpha *(t-\beta)))+1)$, $\alpha=\frac{1}{4}, \beta=22$. This modification (in combination with $V_{\text {disch }}=0.3 \mathrm{~V}$ ) results in reaching the value $c_{\text {eff, level }}$ even more rapidly. Note that the theoretical calculations for Fig. 2.6 are based on $\Phi=27.5 \mathrm{~mL} / \mathrm{min}$ (for the stack of 4 cells), instead of $\Phi=30 \mathrm{~mL} / \mathrm{min}$, to make the theory-lines fit the data somewhat better. Thus these calculations must not be considered as exact, but as illustrative. Fig. 2.6B shows that considering only the first $100 \mathrm{~s}$ of charging, the dynamic charge efficiency $\Lambda^{*}$ increases significantly with discharge voltage. As the total charge input is constant $(41 \mathrm{C})$, this implies that the desalination also increased in the same way. In this case, however, the energy consumption is not inversely proportional to $\Lambda^{*}$ as it is in Fig. 2.5, because the cell voltage is no longer constant. Instead, 

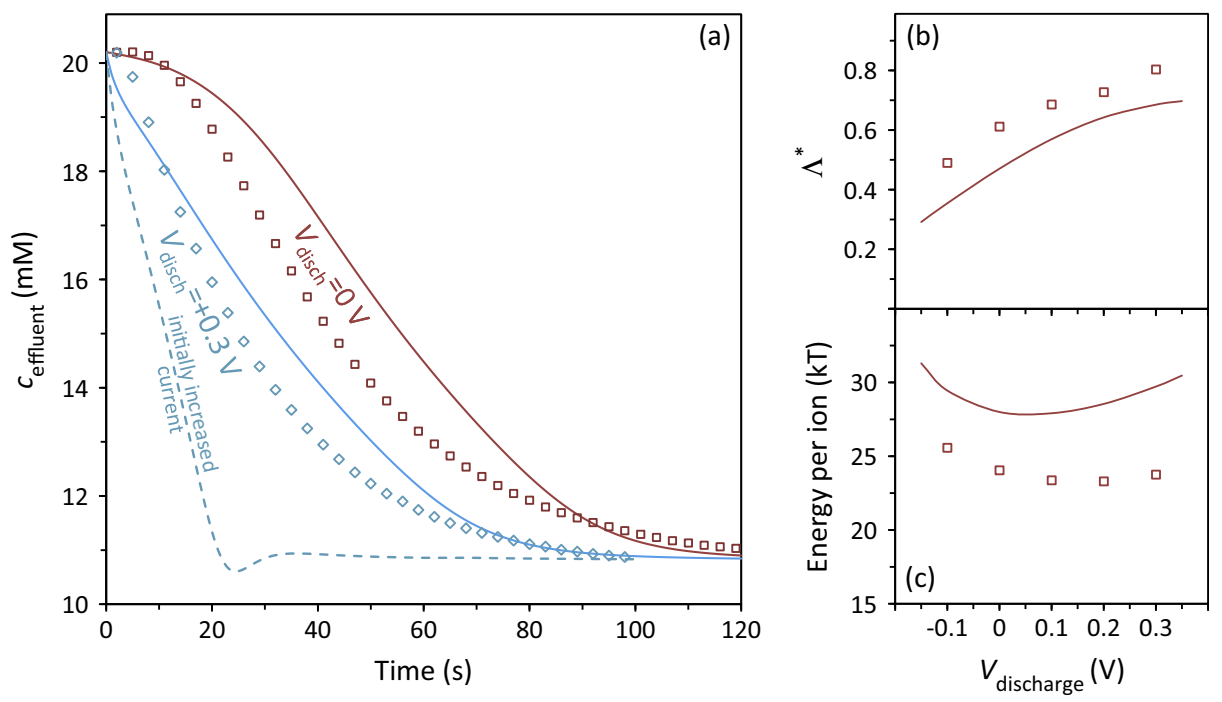

Fig. 2.6: Constant-current CDI operation. a) Effluent salt concentration as function of $V_{\text {disch }}$ (data represented by dots; theory calculations by lines). b) Dynamic charge efficiency $\Lambda^{*}$ vs. $V_{\text {disch }}$ c) Electrical energy input per ion removed $(\mathrm{kT})$ vs. $V_{\text {disch }}$. $\left(I_{\mathrm{ch}}=30.6 \mathrm{~A} / \mathrm{m} 2, c_{\infty}=20 \mathrm{mM}\right.$, electrode thickness $\left.320 \mu \mathrm{m}\right)$.

Fig. 2.6C shows how for CC operation the energy consumption (without energy recovery) is rather invariant with $V_{\text {disch }}$. Interestingly, both theory and data predict a shallow minimum in energy consumption, though at a different value of $V_{\text {disch. }}$.

\subsection{Conclusions}

In this Chapter, we have demonstrated that the charge efficiency of a CDI cycle can be improved by increasing the discharge voltage $V_{\text {disch }}$. In constant voltage $(\mathrm{CV})$ operation, $V_{\text {disch }}$ up to $0.3 \mathrm{~V}$ was promising since charge efficiency was significantly enhanced while salt adsorption only minutely decreased. Another important finding was kinetics in constant-current (CC) operation: the constant level in effluent salt concentration, which is the key advantage of $\mathrm{CC}$ operation, was more rapidly reached when $V_{\text {disch }}$ was increased to a value higher than zero. For CV operation, the required energy input per ion removed went down significantly when we increased the discharge voltage, for our standard experimental settings by about $14 \%$ without energy recovery during discharge, and by $30 \%$ with perfect energy recovery.

The improved modified (i-mD) Donnan model described the full experimen- 
tal data set of equilibrium salt adsorption and charge to an excellent degree. Including the $\mathrm{i}-\mathrm{mD}$ model in a porous electrode transport theory resulted in a dynamic theory that well illustrates several of the observed dynamic features of ion transfer and charge storage, both for constant-voltage and constant-current operation. In conclusion, our work showed that to increase system performance of CDI, there are many more options open besides developing new electrode materials, or the development of improved membranes. We demonstrate that by carefully adjusting voltage and current levels in a CDI cycle, salt adsorption per unit charge transferred can be significantly increased, as well as the time by which (in CC operation) a constant salt effluent level is reached. 


\section{Resistance identification and rational process design in MCDI}

This Chapter has been published as:

J.E. Dykstra, R. Zhao, P.M. Biesheuvel, A. van der Wal, Resistance identification and rational process design in Capacitive Deionization, Water Research 88 (2016) 358-370. 


\section{Abstract}

Capacitive Deionization (CDI) is an electrochemical method for water desalination employing porous electrodes. To enhance the performance of this technology, identification of electronic and ionic resistances in the CDI cell is important. In present Chapter, we outline a method to identify these resistances. We illustrate our method by calculating the resistances in a CDI cell with membranes (MCDI) and by using this knowledge to improve the cell design. To identify the resistances, we derive a full-scale MCDI model. This model is successfully validated against experimental data and used to calculate the ionic resistances across the MCDI cell. We present a novel way to measure the electronic resistances in a CDI cell, as well as the spacer channel thickness and porosity after assembly of the MCDI cell. We identify that for inflow salt concentrations of $20 \mathrm{mM}$ the resistance is mainly located in the spacer channel and the external electrical circuit, not in the electrodes. Based on these findings, we show that the electrode thickness can be increased without significantly increasing the energy consumption, which has the advantage that the desalination time can be lengthened significantly. 


\subsection{Introduction}

Capacitive Deionization (CDI) is an electrochemical desalination technology which employs porous electrodes to adsorb ions from water [27, 104-106, 110, 120, 122, 137]. Upon applying a voltage difference between two electrodes, cations are adsorbed from the water into the electrically negatively charged electrode (cathode), while anions are adsorbed into the positively charged electrode (anode). During this adsorption step, or charging step, feed water flows through the cell and is desalinated. After the electrodes are saturated, the electrodes are short-circuited and ions are released. This is the desorption or discharge step.

For electrodes based on carbon, as will be considered in this Chapter, during the adsorption step, ions are stored in electrical double layers (EDLs) formed in the electrodes $[29,30]$. For every electron transported from one electrode to the other, in both electrodes a counterion can adsorb in these EDLs, or a co-ion can desorb, see Chapter 2. Whereas the adsorption of counterions is desired and leads to salt adsorption, the desorption of the co-ions is undesired. In order to increase the salt adsorption, a cation exchange membrane (CEM) can be placed in front of the cathode, and an anion exchange membrane (AEM) in front of the anode $[34,36,37,71,102]$. These membranes allow the passage of counterions and block co-ions, and therefore, enhance the desalination performance. The CDI technology including membranes in the cell configuration, is referred to as Membrane Capacitive Deionization (MCDI).

CDI cells with various electrode configurations have been proposed, such as flow-through electrodes [112, 138], flowable carbon slurries [2, 72], or wire-shaped electrodes [80]. In the present Chapter we focus on a cell configuration using static film electrodes, where the water flows between the two planar electrodes. In this flow-by configuration, the ion exchange membranes are placed between the spacer channel and the electrodes, as shown in Fig. 3.1. On the other side of the electrode, current collectors are connecting the electrodes with the external electrical circuit.

To improve the performance of the CDI technology, it is important to identify where are the resistances in the cell [139-141]. To this end, we have to quantify the contribution of the different elements of the cell to the total resistance, and thus, to energy losses. In the present Chapter, we will separate the resistances in two types: I) electronic resistances, which are related to the transport of electrons and thus located in the cables, current collectors, and in the carbon matrix in the electrodes, and II) the ionic resistances, which are related to the ion transport and thus are located in the pores of the electrodes, in the membranes and in the spacer channel. These electronic and ionic resistances result in voltage drops over the different elements of the cell, see Fig. 3.1. 
In the present Chapter, we will address the following issues: I) to present a methodology to identify and quantify the electronic and ionic resistances in the different elements in a CDI cell, and II) to show how such knowledge can be used to rationally make improvements in CDI architecture and operation. As an example, we illustrate our method by calculating resistances in a laboratory scale MCDI cell, i.e. a CDI cell including membranes.

The ionic resistances are mainly dependent on the local salt concentration, i.e., higher salt concentrations result in lower resistances. Therefore, we have to calculate the salt concentration profiles across the spacer channel, the membranes and the electrodes during operation of the MCDI cell, in order to determine the ionic resistances over these elements. The transmission line (TL) theory proposed by de Levie [52] was used by Posey and Morozumi [54] to describe charge transport in a porous electrode, and extended by Johnson and Newman [56] to the case of desalination. However, TL theory is derived for electrodes for which a constant salt concentration can be assumed. Since CDI is typically operated at changing salt concentrations, TL theory is not suitable for CDI and must be extended $[49,142]$. In this extension of TL theory, we combine an accurate model for the structure of the EDL in the micropores of the carbon electrode [49] with a model for the transport of ions across the entire porous carbon electrode. This electrode model is combined with a dynamic transport model for ions across the spacer channel and membranes.

In order to use this model to calculate the ionic resistances in the cell, several input parameters should be determined. In the present Chapter, we will present an experimental procedure to do so, including a novel approach to determine the thickness and the porosity of the compressed spacer channel inside the MCDI cell. Our next step is to verify the model by comparing simulation results with constant current charging/discharge experiments. Thereafter, the validated model is used to predict the resistances across the MCDI cell, and the information obtained from these simulations can be used to come with recommendations for an improved cell design. These recommendations will be experimentally verified. In particular, for the tested cell geometry, one recommendation is that one can double or triple the thickness of the electrodes, and thus have longer periods of desalination, while the energy costs of desalination stay the same.

\subsection{Theory}

In the present Chapter, we combine two models, the improved modified Donnan (i-mD) model, and a transport model. The i-mD model describes the EDLs formed in the carbon micropores and relates the charge density, salt adsorption and potential to one another. The transport model calculates the transport of 


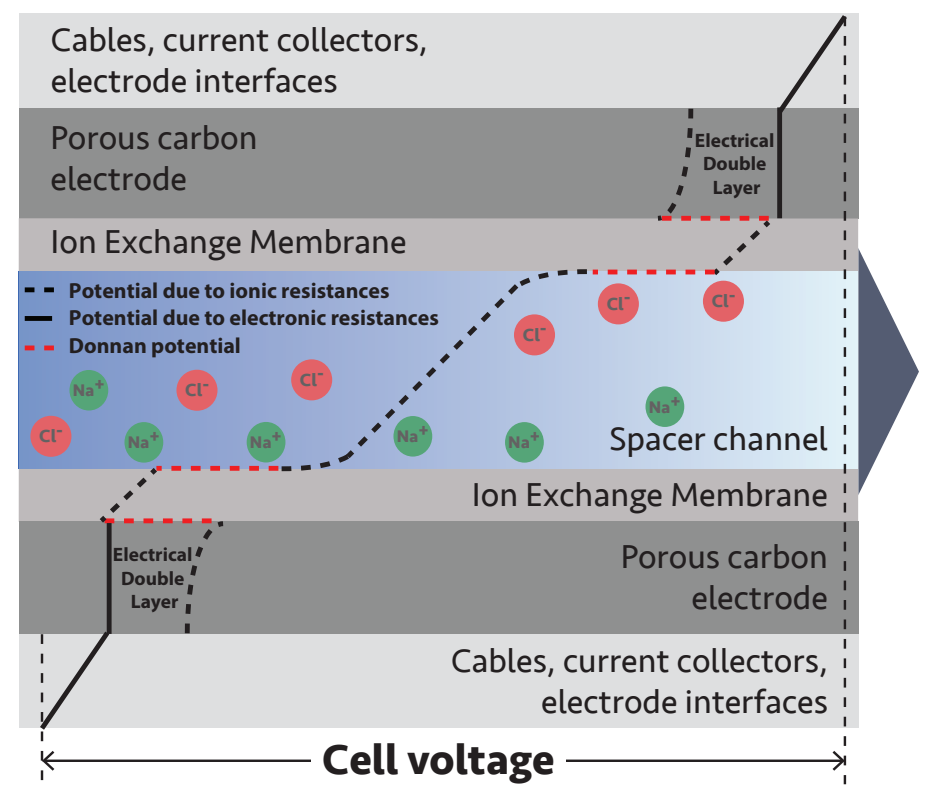

Fig. 3.1: Qualitative overview of the potential profiles across a Membrane Capacitive Deionization cell with carbon electrodes.

ions from the spacer channel, through the membranes, into the porous carbon electrodes; thus the transport is described in the direction perpendicular to the flow channel. In the electrode, we model two different phenomena: I) transport of salt across the electrode through the macropores and, II) simultaneous adsorption of ions into the micropores, where EDLs are formed. The i-mD model is discussed in Section 2.2.1. The transport model is described next.

To derive the transport model, we will make three simplifications. I) We model a symmetric MCDI cell, which means that the anode has the same characteristics, regarding geometrical dimensions and porosity, as the cathode, except for the sign of charge. The same holds for the membranes, i.e. the AEM has the same properties, regarding its dimensions, porosity and membrane charge, as the CEM, except for the sign of the membrane charge. II) We only model the adsorption and transport of $\mathrm{KCl}$, for which we assume the cation and the anion to have the same diffusion coefficient in all elements of the cell. III) We assume that only capacitive processes occur in the electrodes, and no electrochemical reactions take place $[60,99]$. These simplifications allow us to model only half of the cell, so we only consider half of a spacer channel, that is from the spacer midplane to the spacer-membrane boundary, one membrane and one electrode. 


\subsubsection{Porous carbon electrodes}

In the electrodes, we distinguish three different types of pores. Firstly, we consider the micropores, where EDLs are formed and ions are stored. Secondly, we describe the macropores, which are used as transport pathways for ions to go from the membrane-electrode boundary to the micropores. Thirdly, we include mesopores in the model, where neither ions are stored in EDLs nor ions are transported through, but which contain charge-neutral electrolyte, at the same concentration as in the macropores (at the same position in the electrode).

The molar flux of ions through the macropores, subject to diffusional and migrational forces, is given by the Nernst-Planck equation

$$
J_{i}=-D_{i} \cdot\left(\frac{\partial c_{\mathrm{mA}, i}}{\partial x}+z_{i} \cdot c_{\mathrm{mA}, i} \cdot \frac{\partial \phi_{\mathrm{mA}}}{\partial x}\right)
$$

where subscript $i$ refers to ion type $i$, and where $J_{i}$ is the molar ion flux in $\mathrm{mol} / \mathrm{m}^{2} / \mathrm{s}, D_{i}$ the ion diffusion coefficient in $\mathrm{m}^{2} / \mathrm{s}, c_{\mathrm{mA}, i}$ the ion concentration in the macropores in $\mathrm{mol} / \mathrm{m}^{3}, \phi_{\mathrm{mA}}$ the dimensionless potential in the macropores, and $x$ the coordinate running from the membrane-electrode boundary to the electrode-current collector boundary. We neglect tortuosity effects.

At each point in the electrode, we evaluate the ion mass balance

$$
\frac{\partial}{\partial t}\left(\left(p_{\mathrm{mA}}+p_{\mathrm{meso}}\right) \cdot c_{\mathrm{mA}, i}+p_{\mathrm{mi}} \cdot c_{\mathrm{mi}, i}\right)=-p_{\mathrm{mA}} \cdot \frac{\partial J_{i}}{\partial x}
$$

where $p_{\mathrm{mA}}, p_{\text {meso }}$, and $p_{\mathrm{mi}}$ are the macro-, meso- and microporosity of the electrode, and $c_{\mathrm{mi}, i}$ is the ion concentration in the micropores.

Furthermore, we need the electroneutrality condition for the macro- and mesopores

$$
\sum_{i} z_{i} \cdot c_{\mathrm{mA}, i}=0
$$

where $\Sigma_{i}$ describes a summation over all ions in the system, i.e. $\mathrm{K}^{+}$and $\mathrm{Cl}^{-}$, just as in Eqs. (3.4), (3.16) and (3.17).

The micropore charge density, $\sigma_{\mathrm{mi}}$, and micropore ions concentration, $c_{\text {ions,mi }}$ are defined as

$$
\begin{aligned}
\sigma_{\mathrm{mi}} & =\sum_{i} z_{i} \cdot c_{\mathrm{mi}, i} \\
c_{\mathrm{ions}, \mathrm{mi}} & =\sum_{i} c_{\mathrm{mi}, i} .
\end{aligned}
$$

Based on Eqs. (3.1)-(3.4), we derive two balance equations describing the evolution of $\sigma_{\mathrm{mi}}$ and $c_{\mathrm{mA}}$ as function of time. First, we set up the micropore charge balance evaluating $\sigma_{\mathrm{mi}}$ over time by combining Eqs. (3.1)-(3.4), which results in

$$
p_{\mathrm{mi}} \cdot \frac{\partial \sigma_{\mathrm{mi}}}{\partial t}=2 \cdot p_{\mathrm{mA}} \cdot D \cdot \frac{\partial}{\partial x}\left(c_{\mathrm{mA}} \cdot \frac{\partial \phi_{\mathrm{mA}}}{\partial x}\right)
$$


where $c_{\mathrm{mA}}$ is the salt concentration in the macro- and mesopores, which is equal to the ion concentration of $\mathrm{K}^{+}$and that of $\mathrm{Cl}^{-}$there. Parameter $D$ is the diffusion coefficient of $\mathrm{K}^{+}$and $\mathrm{Cl}^{-}$, which are assumed to be equal to one another.

Second, Eqs. (3.1) and (3.3) are substituted in Eq. (3.2), and summed over the cat- and the anion, resulting in the salt mass balance

$$
\frac{\partial}{\partial t}\left(2 \cdot\left(p_{\mathrm{mA}}+p_{\mathrm{meso}}\right) \cdot c_{\mathrm{mA}}+p_{\mathrm{mi}} \cdot c_{\mathrm{ions}, \mathrm{mi}}\right)=2 \cdot p_{\mathrm{mA}} \cdot D \cdot \frac{\partial^{2} c_{\mathrm{mA}}}{\partial x^{2}} .
$$

Across the electrode, at each position, $\phi_{\mathrm{mA}}$ is related to the potential in the carbon matrix, $\phi_{1}$, the Donnan potential, $\Delta \phi_{\mathrm{D}}$, and the Stern potential, $\Delta \phi_{\mathrm{S}}$. These potentials follow from the i-mD model (see Section 2.2.1). The potentials $\phi_{\mathrm{mA}}$ and $\phi_{1}$ are related to $\Delta \phi_{\mathrm{D}}$ and $\Delta \phi_{\mathrm{S}}$ by

$$
\phi_{1}-\phi_{\mathrm{mA}}=\Delta \phi_{\mathrm{D}}+\Delta \phi_{\mathrm{S}}
$$

In this Thesis, we assume that there is no electronic resistance across the electrode, see Box 3.1. Consequently, the measured electronic resistance can only be located in the external circuit. The external circuit includes the cables, current collectors, and the interface between the current collectors and electrodes. From now on, we call the sum of these resistances the external electronic resistance, EER, with dimension $\Omega \mathrm{m}^{2}$. The assumption that there is no electronic resistance in the electrodes leads to a position-invariant value of $\phi_{1}$, and allows us to relate $\phi_{1}$ to the cell voltage (see Fig. 3.1), $V_{\text {cell }}$, by

$$
V_{\text {cell }}=2 \cdot \phi_{1} \cdot V_{\mathrm{T}}+I \cdot \mathrm{EER}
$$

where $I$ is the current density $\left(\mathrm{A} / \mathrm{m}^{2}\right)$. The thermal voltage, $V_{\mathrm{T}}$, is given by $V_{\mathrm{T}}=R \cdot T / F$, where $R$ is the gas constant $(8.314 \mathrm{~J} /(\mathrm{mol} \cdot \mathrm{K})), T$ temperature $(\mathrm{K})$ and $F$ Faraday's constant $(96485 \mathrm{C} / \mathrm{mol})$. Note that Eq. (3.8) requires the assumption of symmetry, which allows us to model only half of the cell with the potential at the spacer midplane set to zero.

At the $E / C$ interface, we apply the boundary conditions

$$
\left.\frac{\partial c_{\mathrm{mA}}}{\partial x}\right|_{E / C}=0,\left.\frac{\partial \phi_{\mathrm{mA}}}{\partial x}\right|_{E / C}=0
$$

while those at the $M / E$ interface will be described in Section 3.2.3.

\subsubsection{Spacer channel}

Our model for the spacer channel is as follows. In the flow direction, we describe the development of concentration profiles between the in- and outflow of the cell. To that end, we divide the spacer channel in $M$ sequential mathematical sub-cells, 
see Fig. 2 in Ref. [35]. In this approach, the electrolyte flows downstream from sub-cell $i$ into sub-cell $i+1$; therefore, the inflow salt concentration in sub-cell $i+1$ equals the effluent concentration of sub-cell $i$. The effluent salt concentration of the MCDI cell is equal to the concentration in sub-cell $M$. For every sub-cell, we calculate the transport of salt and charge into the electrodes, and the salt concentration in the spacer channel, using Eqs. (3.5) and (3.6).

Also, in the spacer channel concentration profiles develop between the spacer midplane and the membrane, thus in the direction perpendicular to the flow direction. To model these concentration profiles, we set up the following salt mass balance to be solved in each sub-cell of the spacer channel

$$
p_{\mathrm{sp}} \cdot \frac{\partial c_{\mathrm{sp}}}{\partial t}=p_{\mathrm{sp}} \cdot D \cdot \frac{\partial^{2} c_{\mathrm{sp}}}{\partial x^{2}}+\Gamma_{\mathrm{sp}}
$$

where $c_{\mathrm{sp}}$ and $p_{\mathrm{sp}}$ are the salt concentration and porosity of the spacer channel, and where the coordinate $x$ runs from the spacer midplane to the edge with the membrane. Dependent on the experiment, $\Gamma_{\mathrm{sp}}$ is one of the following:

- In case we model experiments without flow through the spacer channel (Section 3.4), $\Gamma_{\mathrm{sp}}$ describes a diffusive transport of salt into the spacer channels from a volume outside (the cells), $V_{\text {out }}$. In this case $\Gamma_{\mathrm{sp}}$ is given by

$$
\Gamma_{\mathrm{sp}}=\gamma\left(c_{\mathrm{out}}-c_{\mathrm{sp}}\right)
$$

where $\gamma$ describes the rate of ion diffusion, and $c_{\text {out }}$ is the concentration in $V_{\text {out }}$, which is constant, equal to the initial value of $c_{\mathrm{sp}}$.

- In case we model experiments with flow through the spacer channel (Sections 3.5 and 3.6), $\Gamma_{\mathrm{sp}}$ is given by

$$
\Gamma_{\mathrm{sp}}=\frac{\Phi_{\mathrm{v}} \cdot M}{A_{\mathrm{cell}} \cdot L_{\mathrm{sp}}}\left(c_{\text {inflow }}-c_{\mathrm{sp}}\right)
$$

where $\Phi_{\mathrm{v}}$ is the water flowrate through the cell and $A_{\text {cell }}$ the surface area of one electrode. For sub-cell $i=1$, the salt concentration $c_{\text {inflow }}$ is equal to the inflow concentration of the MCDI cell, while for $i>1, c_{\text {inflow }}$ is equal to the the value for $c_{\mathrm{sp}}$ of sub-cell $i-1$. Note that we assume an equal flowrate (water velocity), at any point in the spacer channel, i.e., independent of $x$.

At the spacer midplane $\left(S^{*}\right)$, we apply the boundary condition

$$
\left.\frac{\partial c_{\mathrm{sp}}}{\partial x}\right|_{S^{*}}=0 .
$$

For the spacer - membrane interface, boundary conditions are described in Section 3.2.3. 
The current density, $I$, is related to the ionic resistance over the spacer channel, $R_{\mathrm{sp}}$, with dimension $\Omega \mathrm{m}^{2}$, by

$$
R_{\mathrm{sp}} \cdot I=2 \cdot \Delta \phi_{\mathrm{sp}} \cdot V_{\mathrm{T}}
$$

where $\Delta \phi_{\mathrm{sp}}$ is the potential difference over half the spacer channel. $R_{\mathrm{sp}}$ is given by

$$
R_{\mathrm{sp}}=\frac{L_{\mathrm{sp}} \cdot V_{\mathrm{T}}}{2 \cdot p_{\mathrm{sp}} \cdot D \cdot F \cdot<c_{\mathrm{sp}}>}
$$

where $\left\langle c_{\mathrm{sp}}\right\rangle$ is the average salt concentration in the spacer channel.

\subsubsection{Membranes}

Across the membranes, we evaluate the total ion concentration, $c_{\mathrm{T}, \mathrm{m}}$, which is the summation of the concentration of counterions and co-ions defined per open volume [143-145]. For the membranes, we can set up the salt mass balance

$$
p_{\mathrm{m}} \cdot \frac{\partial c_{\mathrm{T}, \mathrm{m}}}{\partial t}=-p_{\mathrm{m}} \cdot \sum_{i}\left(\frac{\partial J_{i, \mathrm{~m}}}{\partial x}\right)
$$

where $p_{\mathrm{m}}$ is the porosity of the membrane, and $x$ the location in the membrane, running from the spacer-membrane boundary, $S / M$, to the membrane-electrode boundary, $M / E$. Flux $J_{i, \mathrm{~m}}$ is given by Eq. (3.1) (with subscript "mA" replaced by " $\mathrm{m}$ ") and diffusion coefficient $D$ in Eq. (3.1) is replaced by $d_{\mathrm{r}, \mathrm{m}} \cdot D$. This factor $d_{\mathrm{r}, \mathrm{m}}$ reduces the diffusion coefficient in the membrane relative to the value in free solution, $D$. In the membranes, electroneutrality is assumed, given by

$$
\sum_{i}\left(z_{i} \cdot c_{\mathrm{m}, i}\right)+\omega \mathrm{X}=0
$$

where $X$ is the membrane charge density in $\mathrm{mol} / \mathrm{m}^{3}$, defined per unit aqueous solution in the membrane, and where $\omega$ is the sign of the membrane charge density, which is positive for an AEM and negative for a CEM. Substituting Eq. (3.1) into Eq. (3.16), and assuming electroneutrality, results in

$$
p_{\mathrm{m}} \cdot \frac{\partial c_{\mathrm{T}, \mathrm{m}}}{\partial t}=p_{\mathrm{m}} \cdot d_{\mathrm{r}, \mathrm{m}} \cdot D \cdot\left(\frac{\partial^{2} c_{\mathrm{T}, \mathrm{m}}}{\partial x^{2}}-\omega X \cdot \frac{\partial^{2} \phi_{\mathrm{m}}}{\partial x^{2}}\right) .
$$

At the $S / M$ and $M / E$ boundaries, we have continuity of the salt flux,

$$
\begin{gathered}
-\left.2 \cdot p_{\mathrm{sp}} \cdot \frac{\partial c_{\mathrm{sp}}}{\partial x}\right|_{S / M}=-\left.d_{\mathrm{r}, \mathrm{m}} \cdot p_{\mathrm{m}} \cdot\left(\frac{\partial c_{\mathrm{T}, \mathrm{m}}}{\partial x}-\omega X \frac{\partial \phi_{\mathrm{m}}}{\partial x}\right)\right|_{S / M} \\
-\left.2 \cdot p_{\mathrm{mA}} \cdot \frac{\partial c_{\mathrm{mA}}}{\partial x}\right|_{M / E}=-\left.d_{\mathrm{r}, \mathrm{m}} \cdot p_{\mathrm{m}} \cdot\left(\frac{\partial c_{\mathrm{T}, \mathrm{m}}}{\partial x}-\omega X \cdot \frac{\partial \phi_{\mathrm{m}}}{\partial x}\right)\right|_{M / E}
\end{gathered}
$$


Current density, $I$, equals $F$ times the molar flux of ionic charge, which is the sum of the molar ion fluxes times the ion valency. For instance, evaluating $I$ in the membrane, at the $M / E$ boundary, we obtain

$$
I=-\left.d_{\mathrm{r}, \mathrm{m}} \cdot D \cdot F \cdot c_{\mathrm{T}, \mathrm{m}} \cdot \frac{\partial \phi_{\mathrm{m}}}{\partial x}\right|_{M / E} .
$$

On the outer surface of the two membranes, the following two conditions apply. I) The concentration $c_{\mathrm{T}, \mathrm{m}}$ relates to the salt concentration just outside the membrane, according to

$$
\begin{gathered}
\left.c_{\mathrm{T}, \mathrm{m}}^{2}\right|_{S / M}=X^{2}+\left.4 \cdot c_{\mathrm{sp}}^{2}\right|_{S / M} \\
\left.c_{\mathrm{T}, \mathrm{m}}^{2}\right|_{M / E}=X^{2}+\left.4 \cdot c_{\mathrm{mA}}^{2}\right|_{M / E} \cdot
\end{gathered}
$$

In addition, II) a Donnan potential develops at the membrane interfaces, given by

$$
\begin{aligned}
& \omega X=\left.2 \cdot c_{\mathrm{sp}}\right|_{S / M} \cdot \sinh \left(\Delta \phi_{D, S / M}\right) \\
& \omega X=\left.2 \cdot c_{\mathrm{mA}}\right|_{M / E} \cdot \sinh \left(\Delta \phi_{D, M / E}\right) .
\end{aligned}
$$

The ionic resistance summed over the two membranes in an MCDI cell, together $R_{\mathrm{m}}$, with dimension $\Omega \mathrm{m}^{2}$, is given by

$$
R_{\mathrm{m}} \cdot I=2 \cdot \Delta \phi_{\mathrm{m}} \cdot V_{\mathrm{T}}
$$

where $\Delta \phi_{\mathrm{m}}$ is defined as $\Delta \phi_{\mathrm{m}}=\left.\phi_{\mathrm{m}}\right|_{M / E}-\left.\phi_{\mathrm{m}}\right|_{S / M}$.

Lastly, we relate the potential in the macropores at the $M / E$ boundary, $\left.\phi_{\mathrm{mA}}\right|_{M / E}$, to the potential over the spacer channel, the Donnan potentials on both sides of the membrane, and the potential drop over the membranes according to

$$
\Delta \phi_{\mathrm{sp}}+\Delta \phi_{\mathrm{D}, S / M}+\Delta \phi_{\mathrm{m}}-\Delta \phi_{\mathrm{D}, M / E}=\left.\phi_{\mathrm{mA}}\right|_{M / E}
$$

\subsection{Characterization: electrode salt adsorption, electronic resistances and spacer properties}

\subsubsection{Electrode salt adsorption}

Our methodology to identify the resistances in the MCDI cell makes uses of the transport model including the i-mD model, to calculate the values for the ionic resistances in the different elements of the MCDI cell. To run this model, we have to determine values of the parameters required in the i-mD model, which are $E$, $v_{\mathrm{mi}}, C_{\mathrm{S}, 0}$ and $\alpha$. Therefore, we conduct constant voltage experiments according to the procedure described in Section 2.3.2, with three modifications: I) we use 
Table 3.1: System and electrode dimensions, operational parameters, and parameters used for theoretical calculations. Parameters obtained from ${ }^{*} 1$ ) equilibrium model fitting, as discussed in Section $3.3 .1 ;{ }^{*} 2$ ) external electrical resistances and spacer properties characterization, as discussed in Section 3.3.1; *3) Ref. [42]; *4) Ref. [144]; *5) transport model calculations, as discussed in Section 3.4.

\begin{tabular}{|c|c|c|c|c|}
\hline \multicolumn{5}{|c|}{ Experimental parameters } \\
\hline$L_{\text {elec }}$ & Electrode thickness & 257 & $\mu \mathrm{m}$ & \\
\hline$L_{\mathrm{m}}$ & Membrane thickness & 160 & $\mu \mathrm{m}$ & \\
\hline$A_{\text {cell }}$ & Electrode geometric surface area & 33.8 & $\mathrm{~cm}^{2}$ & \\
\hline$\Phi_{\mathrm{v}}$ & Flowrate through each cell & 7.5 & $\mathrm{~mL} / \mathrm{min}$ & \\
\hline$N_{\mathrm{s}}$ & Number of cells in the MCDI stack & 4 & & \\
\hline$T$ & Temperature & 295 & $\mathrm{~K}$ & \\
\hline$\rho_{\text {elec }}$ & Electrode mass density & 0.569 & $\mathrm{~g} / \mathrm{mL}$ & \\
\hline \multicolumn{5}{|c|}{ Values for use in the i-mD model } \\
\hline$E$ & Micropore ion-correlation energy & 100 & $\mathrm{kT} \mathrm{mol} \mathrm{m} \mathrm{m}^{-3}$ & ${ }^{* 1}$ \\
\hline$v_{\mathrm{mi}}$ & Micropore volume & 0.40 & $\mathrm{~mL} / \mathrm{g}$ & $*^{*}$ \\
\hline$C_{\mathrm{S}, 0}$ & Stern capacitance in zero-charge limit & 160 & $\mathrm{~F} / \mathrm{mL}$ & $*^{*}$ \\
\hline$\alpha$ & Charge dependence of Stern capacitance & 20 & $\mathrm{~F} \mathrm{~m}^{3} \mathrm{~mol}^{-2}$ & ${ }^{*} 1$ \\
\hline \multicolumn{5}{|c|}{ Electronic resistance and spacer channel properties } \\
\hline EER & External electronic resistance & 7.0 & $\Omega \mathrm{cm}^{2}$ & ${ }^{*} 2$ \\
\hline$L_{\mathrm{sp}}$ & Thickness spacer, after assembly & 316 & $\mu \mathrm{m}$ & $*_{2}$ \\
\hline$p_{\mathrm{sp}}$ & Porosity spacer, after assembly & 0.708 & & ${ }^{*} 2$ \\
\hline$L_{\mathrm{sp}, \text { uncompr }}$ & Thickness spacer, before assembly & 335 & $\mu \mathrm{m}$ & \\
\hline$p_{\mathrm{sp}, \text { uncompr }}$ & Porosity spacer, before assembly & 0.724 & & \\
\hline \multicolumn{5}{|c|}{ Values for use in the transport model } \\
\hline$D$ & $\begin{array}{l}\text { Diffusion coefficient of } \mathrm{K}^{+} \text {, and of } \mathrm{Cl}^{-} \\
\text {in free solution }\end{array}$ & 2.03 & $* 10^{-9} \mathrm{~m}^{2} / \mathrm{s}$ & \\
\hline$p_{\mathrm{mA}}$ & Macroporosity & 0.43 & & ${ }^{* 1}$ \\
\hline$p_{\text {meso }}$ & Mesoporosity & 0.05 & & $*^{* 1}$ \\
\hline$p_{\mathrm{mi}}$ & Microporosity & 0.23 & & $* 1$ \\
\hline$p_{\text {sk }}$ & Fraction skeleton material electrode & 0.29 & & *3 \\
\hline$p_{\mathrm{m}}$ & Membrane porosity & 0.3 & & $*^{*}$ \\
\hline$d_{\mathrm{r}, \mathrm{m}}$ & $\begin{array}{l}\text { Diffusion coefficient in the membrane } \\
\text { relative to the value in free solution }\end{array}$ & 0.05 & & $*_{4}$ \\
\hline$X$ & Fixed membrane charge density & 5 & M & $*_{4}$ \\
\hline$\gamma$ & $\begin{array}{l}\text { Transport coefficient of salt from the } \\
\text { outer volume into the spacer channel }\end{array}$ & 0.26 & $\mathrm{mmol} / \mathrm{s}$ & *5 \\
\hline$M$ & Number of mathematical sub-cells & 1 & & \\
\hline
\end{tabular}


$\mathrm{KCl}$ instead of $\mathrm{NaCl}$ to prepare the solution, II) the inflow salt concentrations are 20 and $100 \mathrm{mM}$, and III) experiments are conducted with different, distinct, values of $V_{\mathrm{ch}}$ of $0.2,0.4,0.6,0.8,1$ and $1.2 \mathrm{~V}$, while the discharge voltage is always set to $0 \mathrm{~V}$. After conducting the experiments, we fit the $\mathrm{i}-\mathrm{mD}$ model to the equilibrium data for salt adsorption, charge density and charge efficiency, see Fig. 3.2.

Fig. 3.2 shows that we find a good fit of the theory to the data using values for $v_{\mathrm{mi}}, E, C_{\mathrm{S}, 0}$ and $\alpha$ as listed in Table 3.1. For the transport model, we also need values for the micro-, meso- and macroporosity, as listed in Table 3.1. To calculate these porosities, we follow the procedure described in Section 2.3.3, where we use values for $v_{\text {mi }}$ and $\rho_{\text {elec }}$ as listed in Table 3.1. For the mass density of the "skeleton" material, $\rho_{\mathrm{sk}}$, we use the value given in Section 2.3.3, and for the micropore volume determined by gas sorption analysis, $v_{G S A}$, we use 0.48 $\mathrm{mL} / \mathrm{g} .1$

\subsubsection{Electronic resistances and spacer properties}

Before we run the transport model to calculate the ionic resistances across the MCDI cell, we will first determine the external electronic resistance, EER, which is located in the cables, current collectors and current collector-electrode interfaces (discussed in Section 3.2, we assume that there is no electronic resistance in the electrodes, see results in Box 3.1 which underpin this assumption.)

As the thickness, $L_{\mathrm{sp}}$, and porosity, $p_{\mathrm{sp}}$, of the spacer channel are of influence to the ionic resistance in the spacer channel, $R_{\mathrm{sp}}$, according to Eqs. (3.14) and (3.15), these parameters should be accurately determined as well. Since the (M)CDI stack (assembly of $N_{\mathrm{s}}$ cells), including the spacer channels, is slightly compressed by exerting a pressure of $\sim 0.30$ bar, the thickness and porosity of the spacer channels after assembly are different from the values in uncompressed condition. In this section, we present a method to determine simultaneously EER as well as $R_{\mathrm{sp}}$. In addition, we show how $p_{\mathrm{sp}}$ and $L_{\mathrm{sp}}$ after assembly can be calculated from $R_{\mathrm{sp}}$.

We measure the resistance over a CDI stack, consisting of $N_{\mathrm{s}}$ cells and thus $N_{\mathrm{s}}$ spacer channels, using a Milliohmmeter, see Box 3.1. This device does not measure an ionic distributed resistance in the electrodes as the electronic electrode resistance is very low, see Box 3.1. Thus, the measured resistance over the CDI stack is a sum of the linear resistances EER and $R_{\mathrm{sp}}$. How can we distinguish

\footnotetext{
${ }^{1}$ Please note: in Section 2.3.3 we found a value for the micropore volume determined by gas sorption analysis, $v_{G S A}$, of $0.67 \mathrm{~mL} / \mathrm{g}$ of activated carbon, which recalculates to $0.57 \mathrm{~mL} / \mathrm{g}$ electrode. In present Chapter, however, we did not perform gas sorption analysis on the activated carbon material, but on the electrodes, and we found a value of $0.48 \mathrm{~mL} / \mathrm{g}$ of electrode.
} 


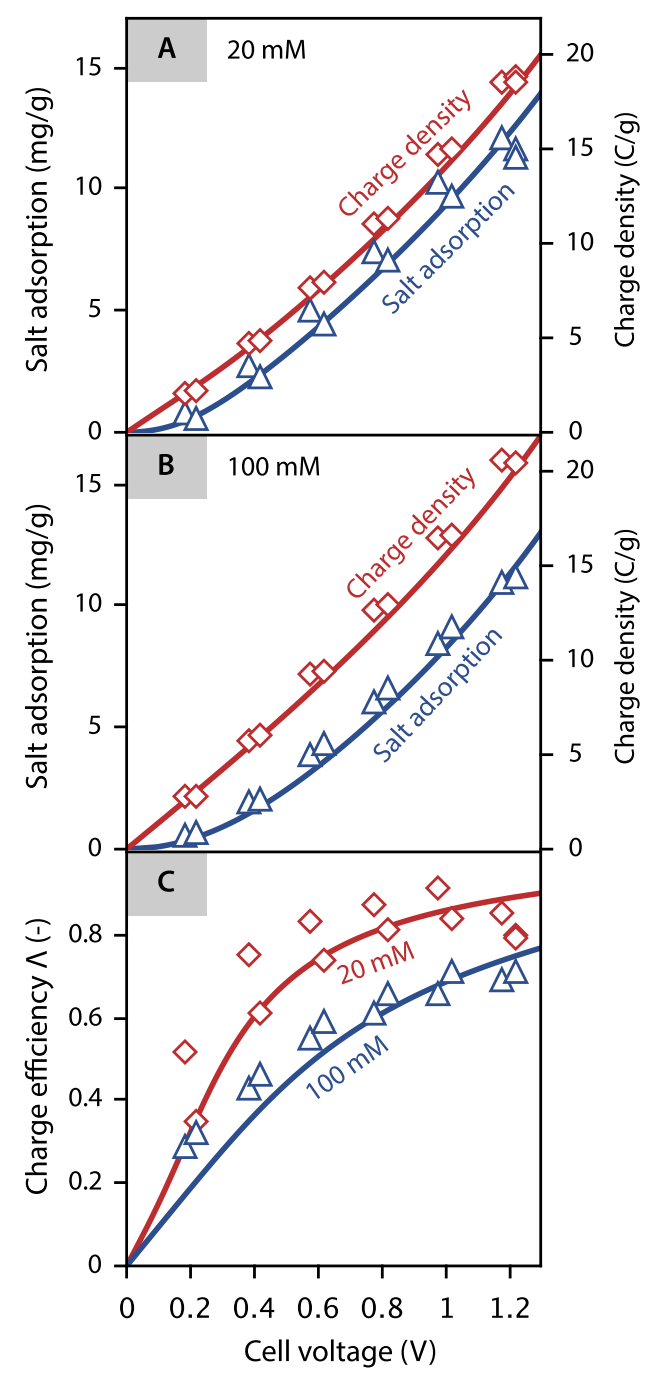

Fig. 3.2: Equilibrium data and theory of the salt adsorption and charge density as function of the cell voltage for a $\mathrm{KCl}$ solution at a concentration of a) $20 \mathrm{mM}$, b) 100 mM. c) Charge efficiency $\Lambda$. Salt adsorption in $\mathrm{mg} / \mathrm{g}$ based on molar mass of $\mathrm{KCl}$. 


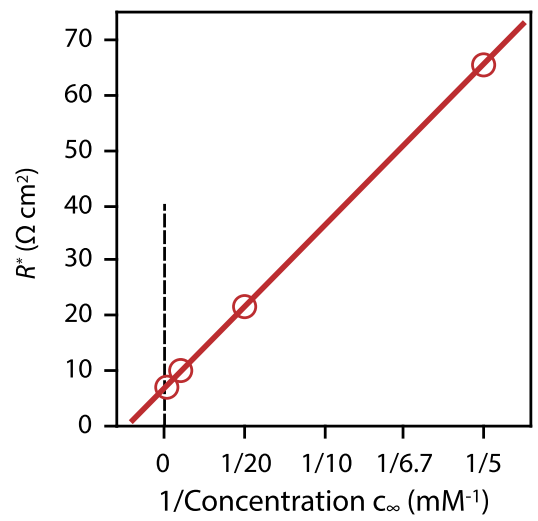

Fig. 3.3: Measured resistance in a CDI cell as function of the electrolyte concentration.

between EER and $R_{\mathrm{sp}}$, and then, based on $R_{\mathrm{sp}}$, calculate $L_{\mathrm{sp}}$ and $p_{\mathrm{sp}}$ ? First we multiply the measured resistance by $N_{\mathrm{s}}$ and $A_{\text {cell }}$ to obtain $R^{*}$, which is the sum of $R_{\text {sp }}$ and EER. Because only $R_{\text {sp }}$ depends on the electrolyte concentration, $c_{\infty}$, we can distinguish between $R_{\mathrm{sp}}$ and EER from the dependence of $R^{*}$ on $c_{\infty}$, see Fig. 3.3. Resistance EER is not dependent on $c_{\infty}$, and follows as the intercept of the curve in Fig. $3.3\left(\mathrm{EER}=7.0 \Omega \mathrm{cm}^{2}\right)$.

The thickness and porosity $L_{\mathrm{sp}}$ and $p_{\mathrm{sp}}$ after assembly can be obtained from Fig. 3.3 as follows. First of all, $R_{\mathrm{sp}}$ is a function of $L_{\mathrm{sp}}$ and $p_{\mathrm{sp}}$ (after assembly) according to

$$
R_{\mathrm{sp}}=\frac{V_{\mathrm{T}} \cdot L_{\mathrm{sp}}}{2 \cdot F \cdot p_{\mathrm{sp}} \cdot c_{\infty} \cdot D} .
$$

Secondly, $p_{\mathrm{sp}}$ and $L_{\mathrm{sp}}$ are related to the uncompressed thickness and porosity of the spacer channel, $L_{\mathrm{sp} \text {, uncompr }}$ and $p_{\mathrm{sp} \text {,uncompr }}$, as listed in Table 3.1 , according to

$$
\left(1-p_{\mathrm{sp}}\right) \cdot L_{\mathrm{sp}}=\left(1-p_{\mathrm{sp}, \text { uncompr }}\right) \cdot L_{\mathrm{sp}, \text { uncompr }} .
$$

Finally, the slope of the curve in Fig. $3.3, \beta$, relates to $R_{\mathrm{sp}}$, by

$$
\beta=R_{\mathrm{sp}} \cdot c_{\infty}
$$

Substituting Eq. (3.26) into Eq. (3.25) and solving the resulting equation with Eq. (3.27), results in $L_{\mathrm{sp}}=316 \mu \mathrm{m}$ and $p_{\mathrm{sp}}=0.708$, see Table 3.1 .

\section{Box 3.1: Electronic resistance in porous electrodes}

One of the underlying assumptions of the transport model is that, within the porous carbon electrodes, the electronic resistance (resistance for flow 
of electronic charge through carbon matrix) is zero. To verify whether this assumption is valid, we measured the electronic resistance over different numbers of electrodes, which are connected via current collectors and cables to an Agilent Milliohmmeter 4338B. This device measures the resistance by applying a sinusoidal potential excitation with a frequency of $1 \mathrm{kHz}$ and measuring the current. In this experiment, a number of wet electrodes is placed on top of each other, without spacer layers, between two current collectors and is slightly compressed by exerting a pressure of 0.29 bar. The results of the measured resistance, multiplied by the electrode surface area of $A_{\text {cell }}=33.8$ $\mathrm{cm}^{2}$, are shown in Fig. 3.4A.

\section{A) Electronic resistance as function} of the number of electrodes

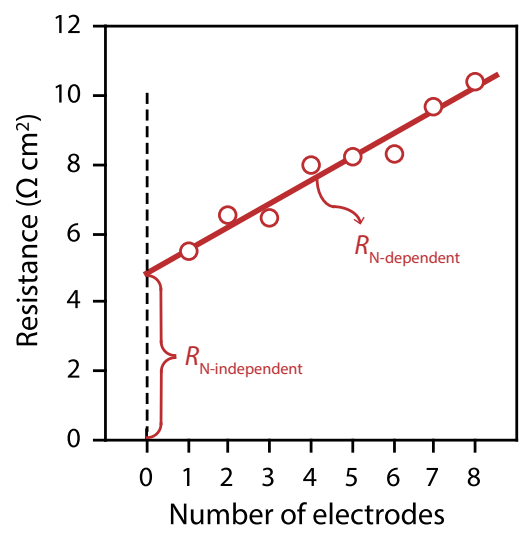

\section{B) Comparison electronic and} ionic resistances electrodes

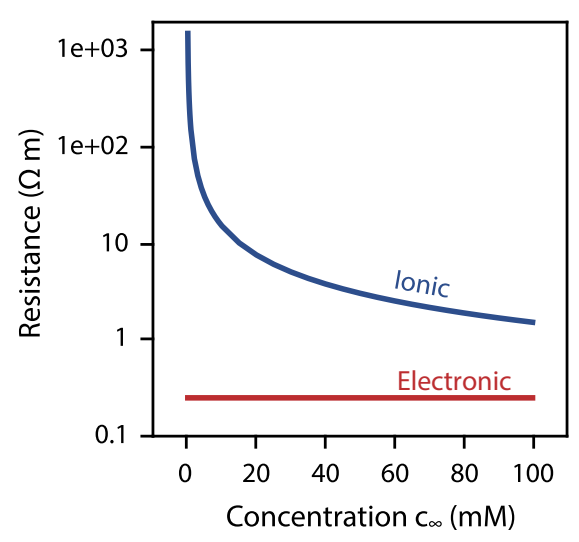

Fig. 3.4: A) Electronic resistance over different numbers of electrodes, and B) Comparison between the electronic and ionic resistance in the electrodes as function of the salt concentration $c_{\infty}$.

As Fig. 3.4A shows, the resistance has a term independent of the number, $N$, of electrodes, given by the intercept, and a term linearly dependent on $N$, given by the slope. The first resistance is in the communal cables and connectors. The second term, which is $N$-dependent, must relate to an electronic resistance in the electrodes themselves, or to an electrode-electrode contact resistance. Therefore, the slope of Fig. 3.4A is equal to the electronic resistance per electrode, $R_{\mathrm{N} \text {-dependent }}$, which is $0.67 \Omega \mathrm{cm}^{2} /$ electrode. Dividing $R_{\mathrm{N} \text {-dependent }}$ by the thickness of one electrode $(L=254 \mu \mathrm{m})$, results in an electronic resistance of $0.26 \Omega \mathrm{m}$, as shown in Fig. 3.4B. We can compare this value with the ionic resistance in the electrodes, $R_{\text {ionic,electrodes, }}$ 
as given by

$$
R_{\text {ionic,electrodes }}=\frac{V_{\mathrm{T}}}{2 \cdot p_{\mathrm{mA}} \cdot c_{\infty} \cdot D \cdot F}
$$

where $V_{\mathrm{T}}, p_{\mathrm{mA}}, D$ and $F$ refer to parameters as used in section 3.2 and listed in Table 3.1.

In Fig. 3.4B we compare the electronic and ionic resistance in the electrodes, as function of $c_{\infty}$. We observe that, for $c_{\infty}=20 \mathrm{mM}$, which is the inflow concentration in the experiments presented in Sections 3.5 and 3.6, the ionic resistance in the electrode is a factor 30 higher than the electronic resistance. This ratio drops with increasing $c_{\infty}$ but even at $100 \mathrm{mM}$, the ratio is still a factor of 10. Consequently, we consider the assumption of zero electronic resistance in the porous carbon electrodes to be valid.

\subsection{Verification: charging and discharge dynamics}

In the previous section, we determined the values of parameters required to describe the salt adsorption in the EDLs of the porous carbon electrodes, as well as the external electronic resistance of the CDI cell and the compressed thickness and porosity of the spacer channel, as listed in Table 3.1. These parameters serve as input for the transport model, which calculates the concentration profiles across the spacer channel, the membranes, and the electrodes, and moreover, the ionic resistances in these different elements. However, before we run the model, we have to verify whether the transport model fits with experimental data.

To that end, we conduct constant current charging/discharge (CCCD) experiments for CDI and MCDI, with and without flowing an electrolyte through the cell (F-CCCD and NF-CCCD respectively), see Fig. 3.5. The experimental scheme is as follows. Before we start the experiment, we flush a salt solution with a concentration of 500, 100 or $20 \mathrm{mM} \mathrm{KCl}$ through the cell and we short-circuit the cell. For the NF-CCCD experiments (Fig. 3.6 A-E), we stop the pump after about $10 \mathrm{~min}$, while for the F-CCCD experiments we leave the pump running with a flowrate of $7.5 \mathrm{~mL} / \mathrm{min}$ per cell. Thereafter, we apply a constant current to charge the cell until a cell voltage of $1.4 \mathrm{~V}$ is reached, and then, we apply the reversed current to discharge the cell until we reach a cell voltage of $0 \mathrm{~V}$. This cycle, of charging and discharging the cell, is repeated 4 times to reach the dynamic equilibrium, which means that the cell voltage signal as function of time of the last cycle is equal to that of the previous cycle. Experiments were run at system currents of $0.25,0.5,0.75$ and $1 \mathrm{~A}$ during the charging step and the reversed current during the discharge step. With the cell area of $A_{\text {cell }}=33.8$ 


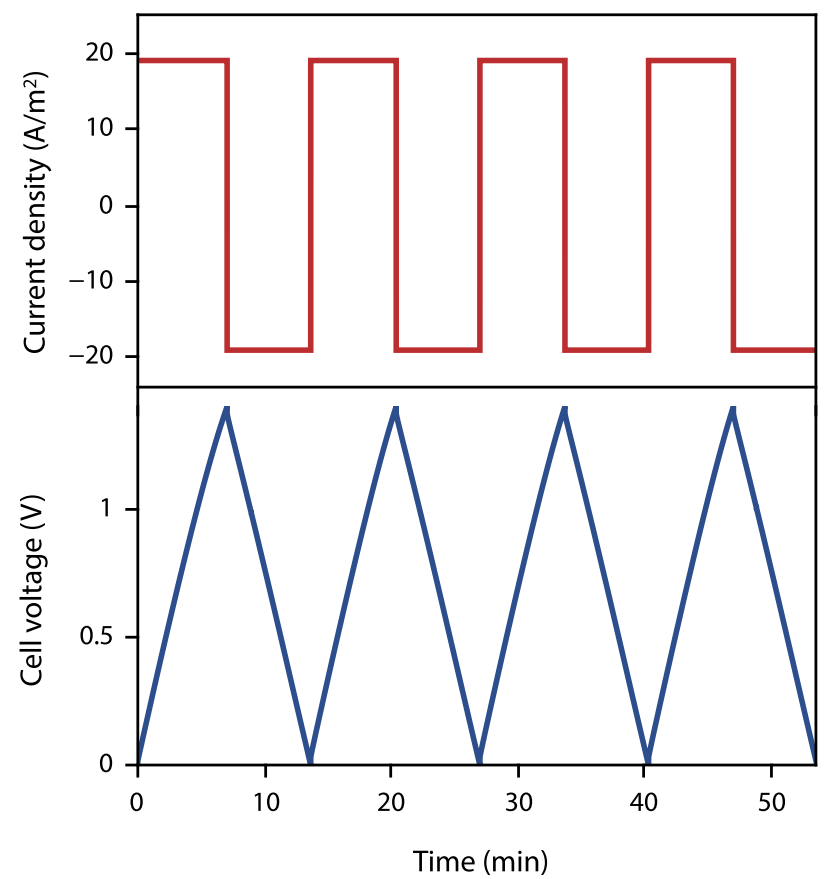

Fig. 3.5: Current density and cell voltage signal of constant current charging and discharge cycles in a CDI cell without flow (NF-CCCD), and with $c_{\infty}$,ini $=500 \mathrm{mM}$. The current was $19 \mathrm{~A} / \mathrm{m}^{2}$ during the charging step and $-19 \mathrm{~A} / \mathrm{m}^{2}$ during the discharge step.

$\mathrm{cm}^{2}$ and $N_{\mathrm{s}}=4$ cells, this recalculates to current densities of $I=19,37,56$ and $74 \mathrm{~A} / \mathrm{m}^{2}$.

The experimental limit cycles in an (M)CDI cell for different electrolyte concentrations are compared with the theoretical limit cycles, as calculated with the transport model, in Fig. 3.6. The $x$-axes of the graphs in Fig. 3.6 include breaks, dividing the $x$-axes in two parts with different timescales. For the NF-CCCD experiments, in order to fit the theoretical curves to the data, we had to include a diffusion term, $\Gamma$, in the spacer channel mass balance, Eq. (3.10), to describe some diffusion of salt from $V_{\text {out }}$ into the spacer channel. We found the best fit of the theory with $\gamma$, the transport coefficient, at a value of $\gamma=0.26 \mathrm{mmol} / \mathrm{s}$. Fig. 3.6 shows that for these NF-CCCD experiments, the transport model fits the experimental data well, both for CDI and MCDI, especially at the higher initial salt concentrations $\left(c_{\infty}\right.$,ini $)$ of 100 and $500 \mathrm{mM} \mathrm{KCl}$. However, as observed in Fig. 3.6E, for CDI with the initial salt concentration of $20 \mathrm{mM}$ the fit is not as good: though the cycle time is well predicted, the cell voltage in the plateau region of the charging step is underestimated. 
A) CDI $500 \mathrm{mM} \mathrm{KCl}$

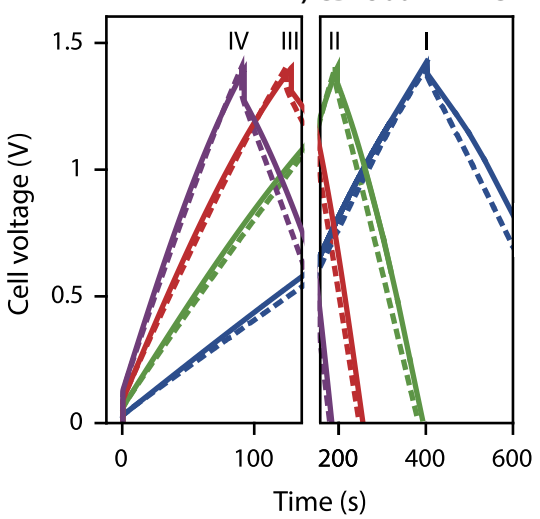

C) CDI $100 \mathrm{mM} \mathrm{KCl}$

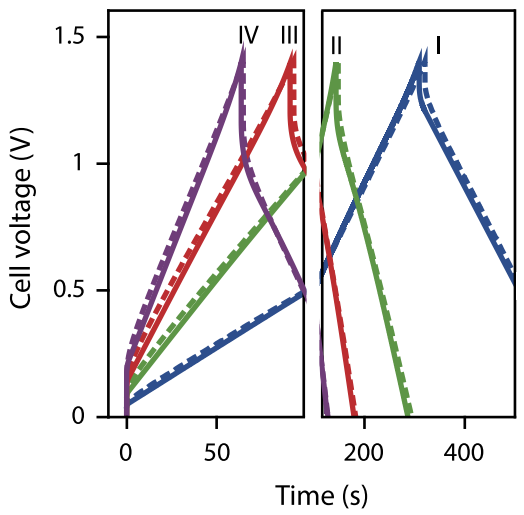

E) CDI $20 \mathrm{mM} \mathrm{KCl}$

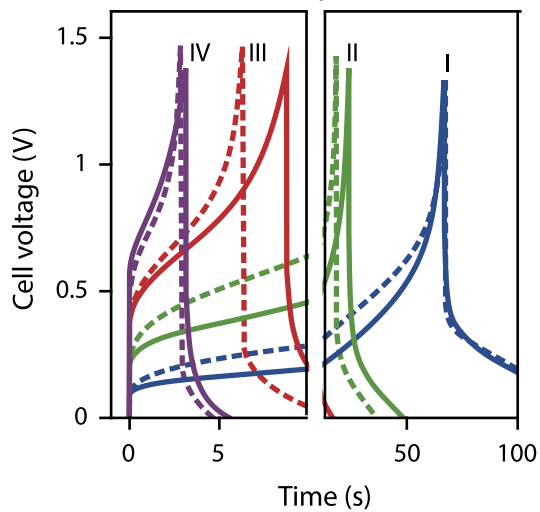

B) MCDI $500 \mathrm{mM} \mathrm{KCl}$

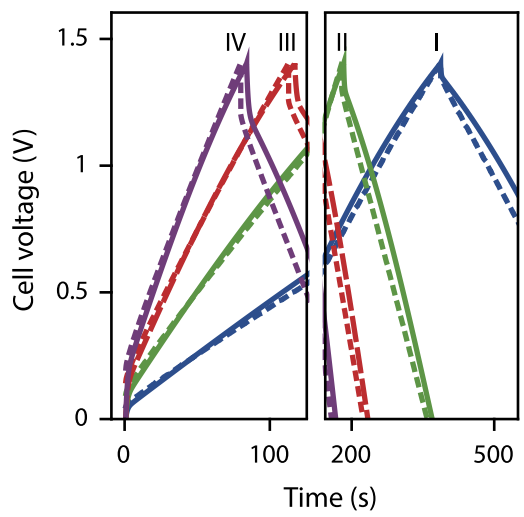

D) MCDI $100 \mathrm{mM} \mathrm{KCl}$

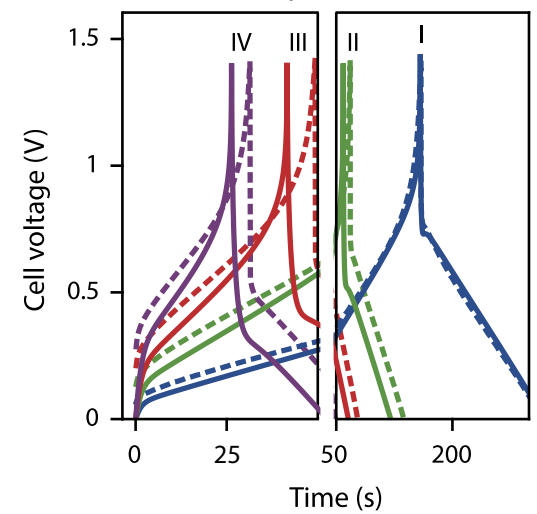

F) MCDI $20 \mathrm{mM} \mathrm{KCl}$

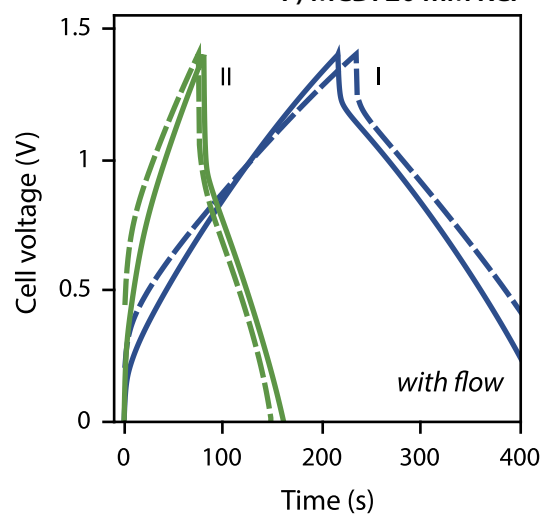

Fig. 3.6: Charge/discharge cycles for CDI and MCDI with initial salt concentrations of 500, 100 and $20 \mathrm{mM} \mathrm{KCl} \mathrm{(A-E,} \mathrm{NF-CCCD),} \mathrm{and} \mathrm{with} \mathrm{an} \mathrm{inflow} \mathrm{salt} \mathrm{concentration}$ of $c_{\text {inflow }}=20 \mathrm{mM}$ (F, F-CCCD). Dashed lines: experimental data. Solid lines: theory. I) $19 \mathrm{~A} / \mathrm{m}^{2}$, II) $37 \mathrm{~A} / \mathrm{m}^{2}$, III) $56 \mathrm{~A} / \mathrm{m}^{2}$ and IV) $74 \mathrm{~A} / \mathrm{m}^{2}$. 
As NF-CCCD MCDI experiments with an initial salt concentration of $20 \mathrm{mM}$ $\mathrm{KCl}$ could not be fitted successfully, we report data and theory of F-CCCD experiments $^{2}$, for which $c_{\text {inflow }}=20 \mathrm{mM} \mathrm{KCl}$. The discrepancy between data and theory for MCDI NF-CCCD at $20 \mathrm{mM}$ can be ascribed to the fact that the overlimiting current was reached, a phenomenon not included in our theory [146]. As shown in Fig. 3.6F, the model describes the data accurately for $20 \mathrm{mM}$ F-CCCD experiments.

\subsection{Prediction: resistances across the MCDI cell}

After presenting the methodology to obtain values for the parameters required in the transport model (Section 3.3), and verifying the transport model with experimental data of CCCD experiments (Section 3.4), now we can use the theory to predict the resistances across the MCDI cell during desalination of brackish water. Therefore, we will simulate the limit cycle of an MCDI experiment, with the same operational scheme as shown in Fig. 3.5, the parameters as listed in Table 3.1, a flow of $7.5 \mathrm{~mL} / \mathrm{min} /$ cell, and an inflow salt concentration of $c_{\text {inflow }}=20 \mathrm{mM}$.

Fig. 3.7 shows the salt concentration profiles across the spacer channel and the electrodes, as well as the free salt concentration across the membranes (which is the counterion concentration minus the fixed membrane charge density, equal to the co-ion concentration), at different moments during the adsorption and desorption step. During the adsorption step, the salt concentration in the electrode (macropores) increases, while the salt concentration in the spacer channel goes down at the same time. The reverse is observed during the desorption step.

Fig. 3.7D shows the ionic resistances in the spacer channel, the membranes, and in the electrodes, as well as the EER, all at the start of a desorption step. To approximate the ionic resistance across the two electrodes in an MCDI cell, $R_{\mathrm{mA}}$, with dimension $\Omega \mathrm{m}^{2}$, we use

$$
R_{\mathrm{mA}} \cdot I=2 \cdot \Delta \phi_{\mathrm{mA}} \cdot V_{\mathrm{T}}
$$

where $\Delta \phi_{\mathrm{mA}}$ is $\left.\phi_{\mathrm{mA}}\right|_{E / C}$, the macropore potential at the electrode - current collector boundary $(E / C)$, minus $\left.\phi_{\mathrm{mA}}\right|_{M / E}$, the potential at the membrane-electrode boundary $(M / E)$. Clearly, Fig. 3.7D shows that the ionic resistance across the spacer is the highest of all resistances, while the ionic resistance in the electrodes

\footnotetext{
${ }^{2}$ Compared to the NF-CCCD experiments as shown in Fig. 3.6, for the F-CCCD experiments, the electrodes were thicker $\left(L_{\mathrm{elec}}=266 \mu \mathrm{m}\right.$ instead of $\left.L_{\mathrm{elec}}=257 \mu \mathrm{m}\right)$ and the mass density was lower $\left(\rho_{\text {elec }}=0.395 \mathrm{~g} / \mathrm{mL}\right.$ instead of $\left.\rho_{\text {elec }}=0.569 \mathrm{~g} / \mathrm{mL}\right)$. Therefore, $p_{\mathrm{mA}}, p_{\text {meso }}$ and $p_{\mathrm{mi}}$ have different values from the ones listed in Table 3.1, respectively 0.61, 0.031 and 0.16. These values are also used in the calculations in Figs. 3.8 and 3.9.
} 

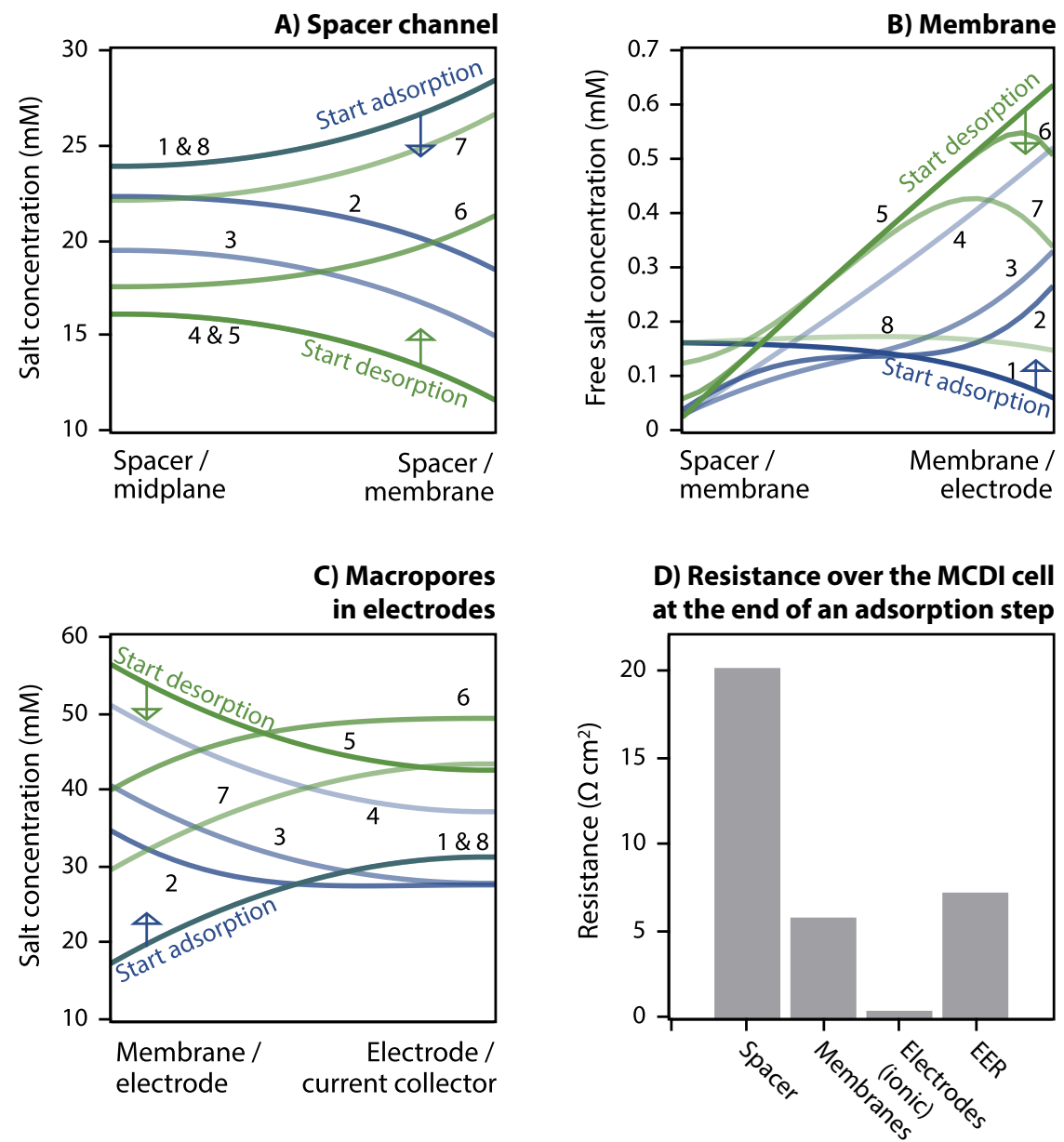

Fig. 3.7: Theoretical prediction of the salt concentration across, and the resistance in, the MCDI cell from the start of the adsorption step (1) until the end of a desorption step $(8)\left(c_{\text {inflow }}=20 \mathrm{mM}, I=19 \mathrm{~A} / \mathrm{m}^{2}\right)$. 
is negligible ${ }^{3}$. The calculated resistance across the membranes $\left(5.6 \Omega \mathrm{cm}^{2}\right)$ is similar to values reported in Refs. [147, 148]. Note that the calculations show that the electronic and ionic resistances do not significantly change during the the cycle. However, values for the resistances shown in Fig. 3.7D can be completely different in other situations, for instance when the salt concentration in the macropores of the electrodes decreases to values close to zero, such as in CDI without membranes.

\subsection{Improved system design: increased electrode thickness}

After identifying that the resistances are mainly located in the spacer channel, the membranes and the external electrical circuit, and not in the electrodes, we can use this information to improve the (M)CDI cell design or operation. Options include the study of the EER, with the aim to reduce it, or the spacer channel resistance (e.g., by using ion-conducting spacers [149] or profiled membranes [150]). Here, as an example, we will focus instead on the electrode. Fig. 3.7D suggests that it should be possible to increase the electrode thickness and consequently the cell capacity (thus requiring less switches between adsorption and desorption), while keeping the energy consumption the same. To test this hypothesis, we performed experiments where we doubled and tripled the electrode thickness in MCDI and we measure the energy requirements.

To this end, we conducted F-CCCD experiments with the same operational scheme and electrodes as those described for the F-CCCD experiments in Section $3.4\left(I=19 \mathrm{~A} / \mathrm{m}^{2}\right)$. The results for the effluent concentration as function of time are shown in Fig. 3.8. As Fig. 3.8 shows, increasing the electrode thickness indeed results in longer cycles. This finding can be explained by the fact that, for the same current density, but with increased thickness, more time is required to reach a cell voltage of $1.4 \mathrm{~V}$, which we set as the maximum voltage before discharge. Comparing the data with the theory, the model closely predicts the desalination degree, but underpredicts the cycle time quite significantly.

For the experiments shown in Fig. 3.8, we calculated the energy consumption by integrating the electrical power (current multiplied by cell voltage) over time, and dividing the resulting value by the salt adsorption in a cycle (in mol).

\footnotetext{
${ }^{3}$ The ionic resistance is calculated according to the aforementioned equation; we will re-analyze this case in Chapter 8 and come to a different conclusion
} 


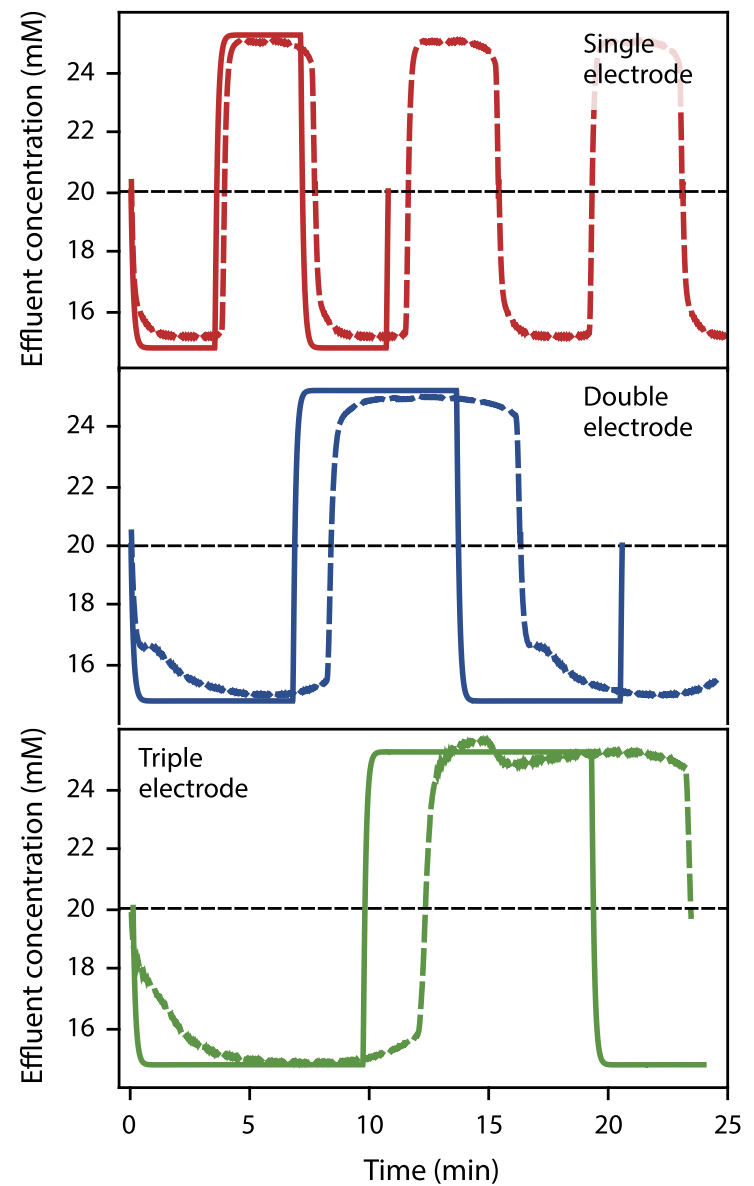

Fig. 3.8: Effluent salt concentration as function of time for MCDI with single, double and triple electrodes $\left(c_{\text {inflow }}=20 \mathrm{mM}, I=19 \mathrm{~A} / \mathrm{m}^{2}\right)$. Dashed lines: experimental data. Solid lines: theory. 


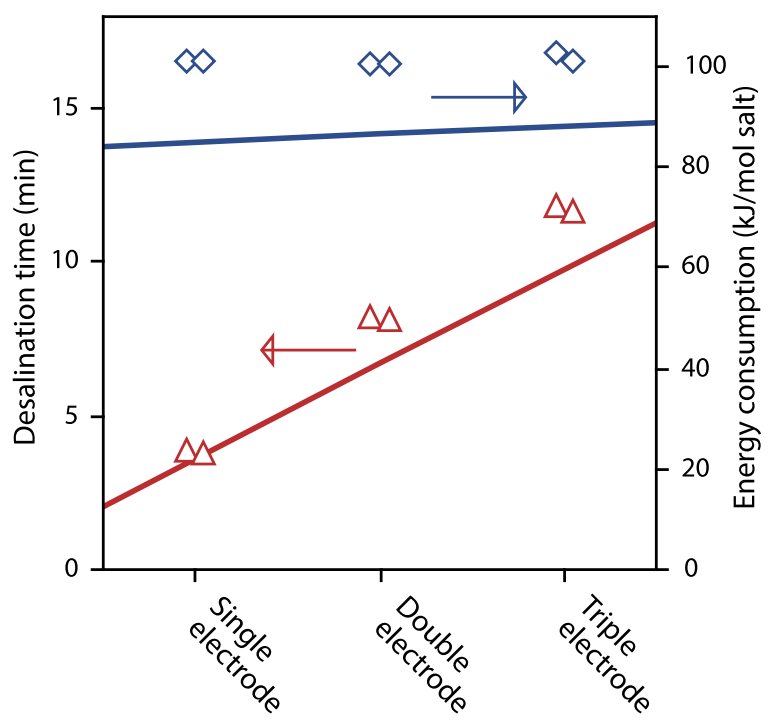

Fig. 3.9: Desalination time and energy consumption for single, double and triple electrodes $\left(c_{\text {inflow }}=20 \mathrm{mM}, I=19 \mathrm{~A} / \mathrm{m}^{2}\right)$. Solid lines: theory.

The resulting values are plotted in Fig. $3.9^{4}$. Note that in this Chapter, we report the energy consumption in dimension $\mathrm{kJ} / \mathrm{mol}$ salt, while in Chapter 2 the dimension $\mathrm{kT} /$ ion was used. One can recalculate values in $\mathrm{kT} /$ ion to values in $\mathrm{kJ} / \mathrm{mol}$ salt by multiplication with a factor $\frac{2 \cdot R \cdot T}{1000} \sim 5$.

Interestingly, the energy consumption of MCDI due to resistances over the different elements of the cell, as presented in Fig. 3.7D, is only $6.1 \mathrm{~kJ} / \mathrm{mol}$ salt. The additional energy requirements are due to the Donnan potentials at the membrane interfaces, and the Donnan and Stern potentials in the electrodes, see also Chapter 8 for further analysis. These energies all relate to the thermodynamics of ion removal and storage, not to transport resistances. Both data and theory presented in Fig. 3.9 indicate that increasing the electrode thickness results in longer periods of desalination, i.e., we have the same desalination rate for a longer period of time with only a very slight increase of the energy consumption.

\footnotetext{
${ }^{4}$ Note that experiments with double and triple electrodes were performed with 2 respectively 1 cell(s) assembled in a stack, instead of the 4 cells in the single electrode experiments. This leads to a different value for the EER. To evaluate energy consumption in Fig. 3.9, we corrected the data to 4 cells using results of a technical study that EER depends on $N_{\mathrm{s}}$ according to EER $=\frac{\alpha}{N_{s}}+\beta$ with $\alpha=17.1 \Omega \mathrm{cm}^{2}$ and $\beta=2.7 \Omega \mathrm{cm}^{2}$.
} 


\subsection{Conclusions}

In the present Chapter, we outlined a methodology to identify the ionic and electronic resistances in Membrane Capacitive Deionization (MCDI). To calculate the ionic resistances, we set up a transport model for the MCDI cell, with a fully discretized spacer channel, membrane and porous carbon electrode, coupled to the improved modified Donnan model. We show that the resulting model can be fitted to experimental data, and that we can use this theory to calculate the ionic resistances across the different elements in the MCDI cell. Furthermore, we presented a novel approach to measure the external electronic resistances, as well as the spacer properties in the MCDI cell.

For the MCDI cell studied, the resistances are mainly located in the spacer channel and in the external electrical circuit, while the resistance in the macropores of the electrodes is significantly lower. This finding predicts, and is also validated by our experimental work, that one can double or triple the electrode thickness without significantly increasing the electrical energy input. Therefore, one can operate the cell with longer periods of desalination, switching less frequently between adsorption and desorption steps, without an extra energy penalty. Furthermore, our results show that, if we want to reduce the resistances in the MCDI cell, we have to focus, in order of priority, on the spacer channel, the external electrical circuit and then on the membranes. 


\section{Energy consumption in CDI - comparison of operational modes}

This Chapter is submitted to Water Research:

J.E. Dykstra, S. Porada, A. van der Wal, P.M. Biesheuvel, Energy consumption in Capacitive Deionization - constant current versus constant voltage operation. 


\section{Abstract}

In the field of Capacitive Deionization (CDI) it has become a common notion that constant current (CC) operation consumes significantly less energy than constant voltage operation (CV). Arguments in support of this claim are that in CC operation the endpoint voltage is reached only at the end of the charging step, and thus the average cell voltage during charging is lower than the endpoint voltage, and that in $\mathrm{CC}$ operation we can recover part of the invested energy during discharge. Though these arguments are correct, in the present Chapter based on experiment and theory, we conclude that in operation of a well-defined CDI cycle, this does not lead to the general conclusion that CC operation is more energy efficient. Instead, we find that without energy recovery there is no difference in energy consumption between CC and CV operation. Including 50\% energy recovery, we find that indeed $\mathrm{CC}$ is somewhat more energy efficient but also in $\mathrm{CV}$ much energy can be recovered. Important in the analysis is to precisely define the desalination objective function, such as that per unit total operational time -including both the charge and discharge steps- a certain desalination quantity and water recovery must be achieved. Another point is that also in CV operation energy recovery is possible by discharge at non-zero voltage. To aid the analysis we present a new method of data representation where energy consumption is plotted against desalination. In addition, we propose that one must analyze the full range of combinations of cycle times, voltages and currents, and only compare the best cycles, to be able to conclude which operational mode is optimal for a given desalination objective. We discuss three methods to make this analysis in a rigorous way, two experimental and one combining experiments and theory. We use the last method and present results of this analysis. 


\subsection{Introduction}

Capacitive Deionization (CDI) is a cyclic method of water desalination using porous electrodes, where salt ions are removed during the charging step, temporarily held inside the electrodes, and released again during discharge [1]. During charging, there is an input of electrical energy, which can partially be recovered during discharge [140]. Many CDI architectures are possible including the use of flowing electrodes [5, 77, 79], chemically modified electrodes [151], redox-active materials $[91,152]$, and the addition of ion-exchange membranes $[37,38,71]$. The most commonly used CDI architecture is the flow-by cell, which employs film electrodes with a spacer channel placed in between, through which the solution flows along the electrodes [2].

For CDI with film electrodes, an important choice that must be made is whether operation will be at constant voltage (CV) or constant current (CC). There can be various criteria to base this choice on, such as the aim for a constant effluent concentration (CC in Membrane CDI), or a low energy consumption of the process. Note that this choice does not need to be made for steady state operation of CDI with flowing electrodes, but is only necessary for cyclic processes. For carbon electrodes (which we consider from this point onward), in literature it is reported that $\mathrm{CC}$ operation leads to a lower energy consumption than $\mathrm{CV}$ operation [36, 97, 153-156]. Kang et al. [153] show that CC-CDI consumes approximately $30 \%$ less energy than CV-CDI for identical electronic charge storage or identical ion removal, without considering energy recovery. Similar differences in energy consumption between $\mathrm{CC}$ and $\mathrm{CV}$ modes without considering energy recovery were reported by Choi [36] and by Han et al. [154]. A much higher advantage of CC over CV implied Ref. [155] where on theoretical grounds CC was considered to have a twice lower energy use than $\mathrm{CV}$ operation. In another study, Qu et al. [97] show that in CC operation with complete energy recovery, the energy consumption can be as low as $28 \%$ of CV operation, i.e., about a factor of three less, and Ref. [156] concludes that energy recovery in CC operation is much more favorable than in CV operation.

Arguments brought forward to explain the lower energy use in CC operation are as follows. First, it is argued that during CC charging the charging voltage is only reached at the end of a charging step, thus on average the cell voltage during charging is lower in $\mathrm{CC}$ operation, and thus the electrical energy consumption is lower. In the ideal case, and with the same (endpoint) voltage, this difference amounts to a factor of two. The other argument is that in CC operation it is possible to recover energy which -it is implied- is not possible during CV operation. This is indeed true when $\mathrm{CV}$ is operated at zero discharge voltage, as has been the classical approach in CV-CDI. However, also in CV operation, 
it is possible to discharge at a non-zero voltage, and thus energy recovery is possible [157].

To compare $\mathrm{CC}$ and $\mathrm{CV}$ operation, one must precisely define the desalination objective, such that per unit total operational time a certain desalination quantity and water recovery must be achieved. Water recovery, WR, is the ratio of the volume (flow) of desalinated water (diluate), over the volume of feed water. For this objective function we must find conditions of operation with lowest energy consumption, which can thereafter be compared for different modes of operation. This is not as straightforward as it may seem: though it is often attempted to compare CC and CV for identical conditions of operation, it is not sufficiently checked whether these conditions result in the same desalination quantity and WR. Choi [36] compares data for CC and CV operation for identical charging voltages, that is, data are compared where the charging voltage applied in CV operation is the same as the endpoint charging voltage in $\mathrm{CC}$ operation. However, for this comparison between any two data points at the same voltage, various other operational properties are different, such as WR, cycle time and salt removal. Kang et al. [153] compare CV and CC operation as function of the salt adsorption capacity of a cycle. Data points are compared at the same value of salt adsorption capacity, and WR of $50 \%$. However, for each data point used in the comparison, the cycle time (the duration of the full charge/discharge cycle) is different. Thus, in Ref. [153] the performance of a single cycle is compared, but not the performance per time period of system operation, and therefore the cycle-averaged salt adsorption rates are not equal. Whereas these papers consider flow-by CDI, Qu et al. [97] perform experiments with flow-through CDI and compare data as function of charge transferred. They show (inset picture Fig. 4 in Ref. [97]) that in their case the amount of charge is close to proportional to salt removal. Furthermore, data were obtained at the same duration of the charging (either CV or CC) and discharge step (CC in all cases). This approach is in line with the proposal of the present Chapter, which is that one must compare data with the same salt removal rate (when averaged over the full cycle). They show that without energy recovery CC has $\sim 35 \%$ less energy consumption, and including energy recovery $\mathrm{CC}$ has up to a factor of three lower energy consumption. Different to our protocol, Qu et al. [97] only analyze a first cycle, and not the "dynamic steady state", but for the longer cycles this should not matter much. Energy consumption was analyzed for flow-by CDI by Zhao et al. in 2012 [71] and compared for CC vs CV operation, CDI vs membrane-CDI, and for different salt concentrations. Conclusions in that paper, however, were based on comparing data with different average salt adsorption rates and thus the conclusions do not rigorously follow.

In order to compare $\mathrm{CC}$ and $\mathrm{CV}$ operation in a fair manner, one has to make 


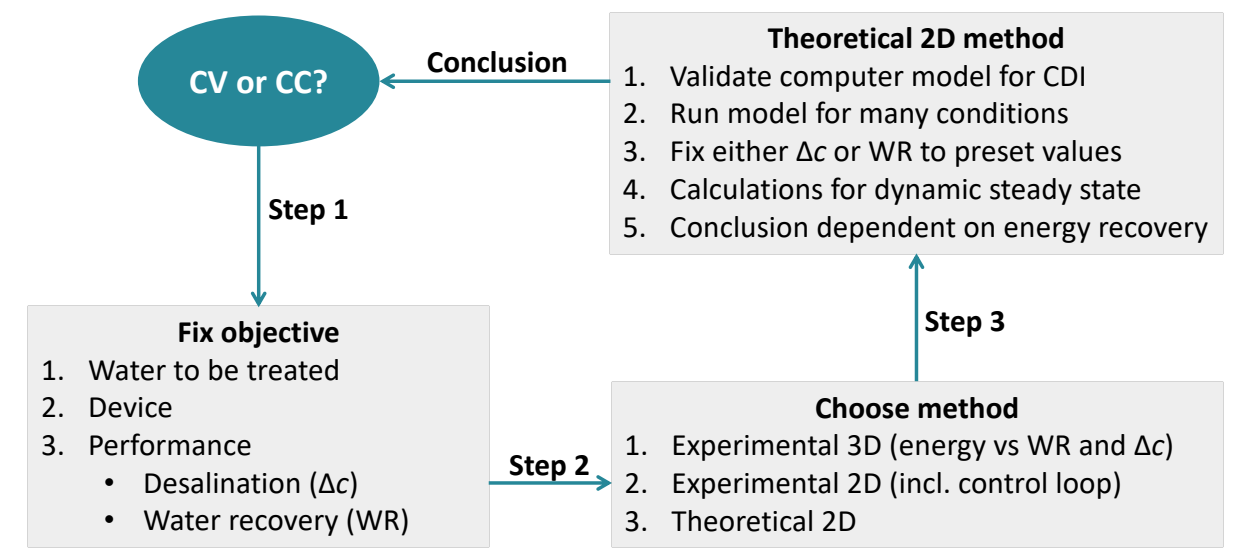

Fig. 4.1: Schematic overview of our methodology to analyze the suitability of a certain operational mode for water desalination by capacitive deionization (CDI), in terms of energy consumption.

sure that the desalination objective of the cycles subject to comparison is the same. The objective is always defined by two parameters:

- water recovery, as define above, and

- a measure of the amount of salt removed. This can be expressed as the average salt adsorption rate, ASAR, which is based on the molar quantity of salt removed from the diluate stream per unit operational time [2]. A second metric that can be used is the difference in salt concentration between the diluate and feed streams, averaged over the duration of the salt adsorption step. We call this difference "desalination," $\Delta c$. In this Chapter we will use $\Delta c$, not ASAR, as our measure for the amount of salt transferred in a CDI cycle.

For each operational mode (CV or $\mathrm{CC}$ ) an infinite number of charging/discharge schemes is possible, which are all defined by different operational parameters. Each scheme results in a desalination cycle with a certain desalination objective and energy consumption. Thus, to reach a certain objective, several operational schemes are possible, and for each scheme the energy consumption can be different. In order to conclude whether $\mathrm{CC}$ or $\mathrm{CV}$ operation performs better for a certain desalination objective, one has to compare for each operational mode the scheme resulting in the desired objective with the lowest energy consumption. This means that one has to conduct, in principle, an infinite number of experiments. Because the desalination objective, defined by WR and $\Delta c$, reached by a certain scheme can only be determined after running the CDI cycle, many experiments must be discarded if we are only interested in one value of WR and $\Delta c$ 
to compare $\mathrm{CC}$ and $\mathrm{CV}$ operation. Instead, we propose that it is better to use all data that are obtained, and thus to compare $\mathrm{CC}$ to $\mathrm{CV}$ operation for a range of values of WR and $\Delta c$. This method would result in a $3 \mathrm{D}$ representation of energy use versus WR and $\Delta c$. This method, which we refer to as "Experimental 3D", see Fig. 4.1, allows for a fair comparison between the operational modes.

Another method, which we refer to as "Experimental 2D", is based on fixing one of the parameters defining the desalination objective, e.g. WR, at a constant value, and to compare the energy consumption of both operational modes as function of $\Delta c$. This means that experiments should be conducted using an operational scheme that results in a pre-defined value of WR, which is not easy, because water recovery is not a parameter that can be imposed directly in an experiment; i.e., it is not an input, but an output of an experiment. Indeed, as we will show, WR can be markedly different from what would be expected based on a calculation involving charging and discharge times. Therefore, experiments have to be designed making use of a control loop: a desalination cycle is conducted with several parameters defining a cycle, then the output of a cycle is analyzed and WR calculated, thereafter the parameters are adapted, and this loop is run through until WR reaches the pre-defined value. Also in this case a very large number of experiments are required to find conditions of minimum energy in a plot of energy consumption versus $\Delta c$.

Instead of these two experimental methods, we make use of a method which combines experiment and theory, see Fig. 4.1. This method consists of performing a limited number of experiments to validate a dynamic CDI model [158-160]. This model is then used to generate a very large number of calculated outputs of energy use, all at a constant pre-defined value of WR (achieved by a numerical search routine) and these "computer data" are compared in a plot for energy consumption versus desalination, $\Delta c$, at fixed values of WR. We refer to this method as "Theoretical 2D".

Thus, to test if CC is energetically preferable to CV operation, according to our method we make use of a validated computer model of a CDI cell, and run a very large number of calculations with varying values of cycle times, current, voltages, etc., all for the same input and device. In this way, we find for each operational mode ( $\mathrm{CV}$ or $\mathrm{CC}$ ) the optimum cycle characteristics and we can control WR exactly, without requiring experiments using a control loop. Optimum values are read off as the lower boundary in a plot of energy versus desalination, $\Delta c$ (at fixed values of WR). These optimum values (i.e. the lower boundaries) can then be compared to derive information on the merit of $\mathrm{CC}$ versus $\mathrm{CV}$ operation with respect to energy use, for conditions where the overall performance of the process is the same. The comparison is made without and with considering energy recovery during discharge. 


\subsection{Theoretical framework}

Theoretical calculations are made using a dynamic CDI model of a single cell, which describes ion electromigration across a spacer channel and through a porous electrode, combined with a suitable EDL-model. For the theoretical equations, we refer the reader to Chapter 3 . In the flow direction, only a single "subcell" is assumed. To describe the EDL, for mathematical simplicity, the improved modified Donnan model is used in the calculations, which was compared with the amphoteric Donnan model $[51,159]$ and was shown to give similar predictions for salt adsorption, charge and charge efficiency, in the relevant range of salinities of this study, see Box 4.1.

\section{Box 4.1: comparing improved modified-Donnan and amphoteric Donnan model}

Theoretical data points reported in this Chapter were calculated using a dynamic CDI model coupled with an EDL model. Whereas the amphoteric Donnan (amph-D) model gives a more accurate description of the EDL, we used the improved modified-Donnan (i-mD) model, because of its mathematical simplicity. To find values for parameters used in the i-mD, we compared results with the amph-D model using parameter settings reported in Ref. [161]), see Fig. 4.2.
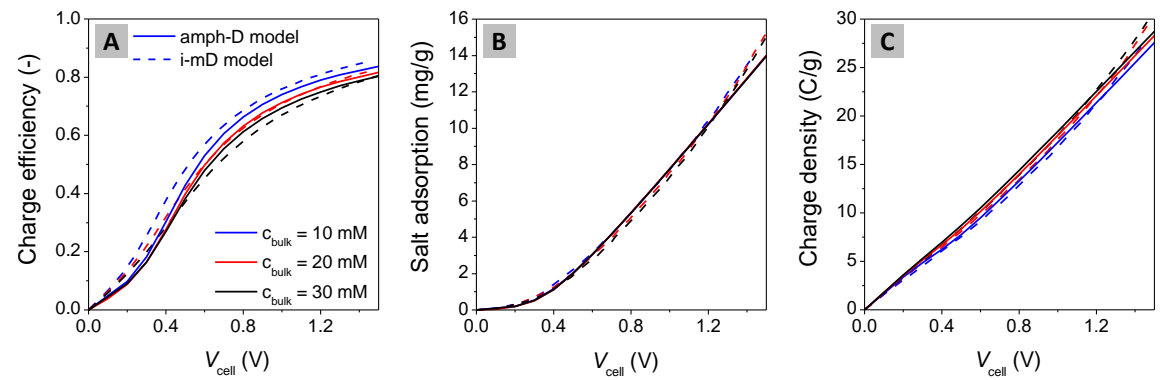

Fig. 4.2: Comparing results of improved modified-Donnan model with the amphoteric Donnan model using parameter settings reported in Ref. [161]). Based on this comparison for $\mathrm{NaCl}$ concentrations of 10,20 and $30 \mathrm{mM}$, parameter values were found for the improved modified-Donnan model as reported in Table 4.1.

We use a diffusion coefficient of the ions (in the open volume of spacer and electrode) of $1.67 \cdot 10^{-9} \mathrm{~m}^{2} / \mathrm{s}$, both for anions and cations, and we neglect tortuosity effects. We assume a plug flow velocity profile in the channel, along the electrodes (fluid velocity is zero in the electrode), and based on this calculate the 
effluent salinity. Values for relevant parameters in this dynamic CDI model are reported in Table 4.1 .

Calculations are always made for the "dynamic steady state" (DSS) by performing at least three cycles, and taking results from the third cycle. Salt adsorption is calculated as the amount of salt released during the period that the effluent salt concentration is higher than that of the inflow (based on the difference in inflow and effluent salt concentration), which is also equal to the adsorption of salt during the other period. Energy input is calculated as the integral of current and cell voltage during the charging step. This is the energy plotted in the graphs labeled "without energy recovery". Subtracting from energy input the energy during discharge, again calculated from the product of current and cell voltage, and including a factor of $50 \%$, we obtain the input for energy plotted in graphs labeled "with $50 \%$ energy recovery."

\subsection{Energy consumption as function of desalination - comparison of operational modes}

We conducted experiments according to the procedures described in Section 4.A. The aim of these experiments was to validate the dynamic CDI model, which we discussed in Section 4.2.

Experimental results are presented in Fig. 4.3 as function of desalination, $\Delta c$, which is the decrease in salt concentration of the diluate compared to the feed stream, averaged over the duration of the desalination step (the time period that the device produces diluate, i.e., that the water has a lower salinity than the feed). For $\mathrm{CV}$ operation, $\Delta c$ increases when we reduce the discharge voltage (at the very right $V_{\text {disch }}=0$ in Fig. $4.3 \mathrm{~A}, \mathrm{~B}$ ), while for $\mathrm{CC}$ operation moving to the right, data points correspond to higher and higher current density. As discussed, we do not use these data to directly come to a conclusion about energy in optimized CC or CV cycles, but use these data to validate the dynamic CDI model.

Fig. 4.3 shows the results of the model validation. For the purpose of this Chapter, which is to outline a method to compare various operational modes for CDI, we found a good qualitative fit between the model and data. We note that to fit the model to the data unrealistic values had to be used for certain parameters in the model, see Table 4.1.

For the data shown in Fig. 4.3, one would perhaps expect that in all cases the water recovery, WR, is $50 \%$, as the charging time was equal to the discharge time, both for $\mathrm{CC}$ and $\mathrm{CV}$ operation. Indeed, Fig. 4.4 shows that for most $\mathrm{CV}$ data this is the case, but it also shows that for some CV and all CC data, WR $<50 \%$. Thus, we find that, although the charging time is equal to the 
Table 4.1: Parameters and their values used in theory.

\begin{tabular}{|c|c|c|c|}
\hline \multicolumn{4}{|c|}{ Operational \& general physical parameters and system dimensions } \\
\hline$\Phi_{\mathrm{V}}$ & Flowrate through each cell & 7.5 & $\mathrm{~mL} / \mathrm{min}$ \\
\hline$T$ & Temperature & 295 & $\mathrm{~K}$ \\
\hline$D$ & $\begin{array}{l}\text { Average diffusion coefficient of } \mathrm{Na}^{+} \text {and } \mathrm{Cl}^{-} \\
\text {in free solution }\end{array}$ & 1.67 & $* 10^{-9} \mathrm{~m}^{2} / \mathrm{s}$ \\
\hline$M_{\mathrm{w}, \mathrm{salt}}$ & Molar mass of $\mathrm{NaCl}$ & 58.4 & $\mathrm{~g} / \mathrm{mol}$ \\
\hline$M$ & Number of mathematical sub-cells & 1 & \\
\hline EER & External electronic resistance ( 4 cells) & & \\
\hline & value used for Figs. 4.3 and $4.4^{1}$ & 40.5 & $\Omega \mathrm{cm}^{2}$ \\
\hline & value used for Figs. $4.5-4.7^{2}$ & 7.0 & $\Omega \mathrm{cm}^{2}$ \\
\hline \multicolumn{4}{|c|}{ Electrode dimensions and porosities } \\
\hline$A_{\text {cell }}$ & Electrode geometric surface area & 33.8 & $\mathrm{~cm}^{2}$ \\
\hline$L_{\text {elec }}$ & Electrode thickness & 200 & $\mu \mathrm{m}$ \\
\hline$p_{\mathrm{mA}}$ & Macroporosity & 0.5 & \\
\hline$p_{\text {meso }}$ & Mesoporosity & 0 & \\
\hline$p_{\mathrm{mi}}$ & Microporosity ${ }^{3}$ & 0.3 & \\
\hline$M_{\text {elec }}$ & Total mass of two electrodes in cell & 1.1 & $\mathrm{~g}$ \\
\hline \multicolumn{4}{|c|}{ Spacer thickness and porosity } \\
\hline$L_{\mathrm{sp}}$ & Thickness spacer, after assembly ${ }^{2}$ & 316 & $\mu \mathrm{m}$ \\
\hline$p_{\mathrm{sp}}$ & Porosity spacer, after assembly ${ }^{2}$ & 0.708 & \\
\hline \multicolumn{4}{|c|}{ EDL parameters } \\
\hline$E$ & Micropore ion-correlation energy ${ }^{4}$ & 320 & $\mathrm{kT} \mathrm{mol} \mathrm{m} \mathrm{m}^{-3}$ \\
\hline$C_{\mathrm{S}, 0}$ & Stern capacitance in zero-charge limit ${ }^{4}$ & 145 & $\mathrm{~F} / \mathrm{mL}$ \\
\hline$\alpha$ & Charge dependence of Stern capacitance ${ }^{4}$ & 20 & $\mathrm{~F} \mathrm{~m} \mathrm{~mol}^{3}$ \\
\hline
\end{tabular}

${ }^{1}$ Follows from fitting experimental data, collected in this Chapter, with theory.

2 Reported in Chapter 3.

${ }^{3}$ Follows from fitting experimental data, collected in this Chapter, with theory. Thus, $p_{\mathrm{mi}}$ does not relate to the micropore volume, $v_{\mathrm{mi}}$, as calculated in Section 3 by matching the i-mD model (used in our theory) with the amph-D model (which was fitted with experimental data in Ref. [161]).

${ }^{4}$ Follows from matching the i-mD model (used in our theory) with the amph-D model (which was fitted with experimental data in Ref. [161]). See Box 4.1 for matching results. 

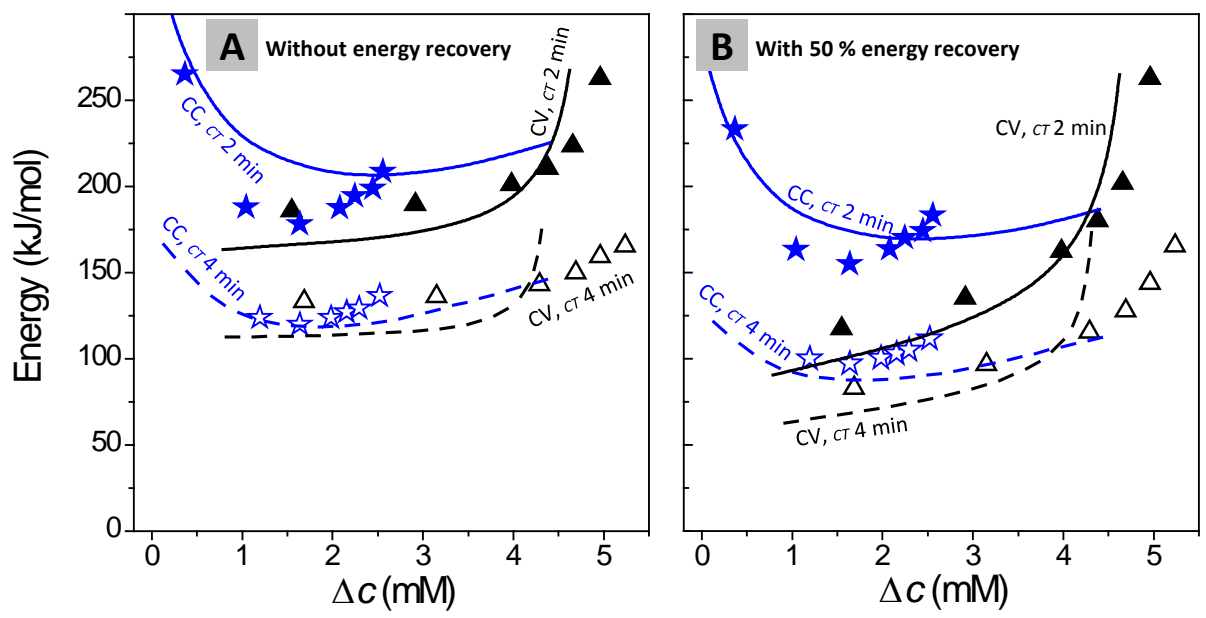

Fig. 4.3: Energy consumption per mole of salt removed as function of desalination, $\Delta c$. Experimental results based on data (CV: triangles, CC: stars) (as presented in Fig. 4.A.2) and results of theoretical calculations using the dynamic CDI model (solid and dashed lines). A) without energy recovery, and B) with energy recovery. The duration of a full cycle, CT, is either 2 or $4 \mathrm{~min}$.

discharge time, especially for the CC experiments, the adsorption time, which is the period with an effluent salt concentration that is lower than that in the feed water, is considerably shorter than the desorption time, which is the period with a higher effluent concentration than that of the feed water. This difference in adsorption and desorption time results, with constant flow rate, in WR $<50 \%$. Thus we cannot simply compare a set of CC and CV experiments, for the reasons outlined before: first, that for a certain operational setting we cannot be sure that we are at the lowest energy for a given desalination objective, and second, in most cases the desalination objective is different (both $\Delta c$ and WR differ between the CC and CV data points).

\subsection{Optimizing desalination performance as function of desalination and WR - comparing constant voltage and constant current}

In Section 4.3 we validated our dynamic model with experimental data and discussed that we use this model to compare the energy consumption, EC, of $\mathrm{CV}$ and $\mathrm{CC}$ operational modes. In the present section we outline our methodology for comparison, and the results thereof. 


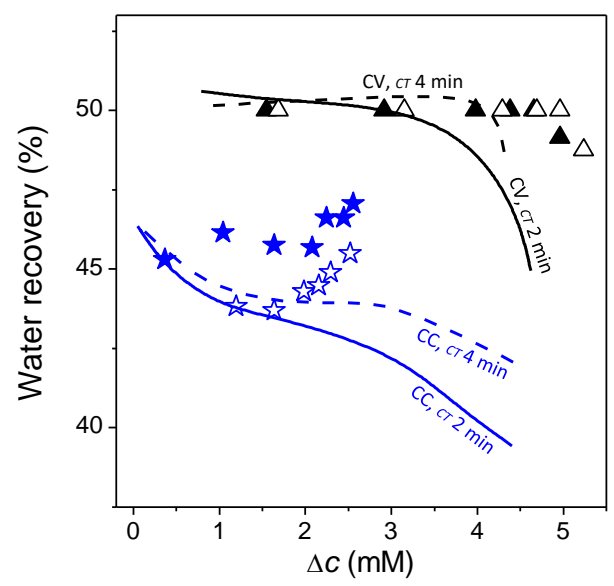

Fig. 4.4: Water recovery as function of desalination, $\Delta c$. Experimental (points) and theoretical results (solid and dashed lines) based on data for which energy consumption is reported in Fig. 4.3. The duration of a full cycle, CT, is either 2 or $4 \mathrm{~min}$.

To find optimum performance, three types of constraints must be considered: the flow rate and composition of the inflowing water, the cell itself (mass of electrodes, dimensions), and the desalination objective. With all of these constraints fixed, we can analyze whether one mode of operation has a higher energy use than another mode. For a different input, cell, or objective, the conclusion can be quite different. Calculations are based on the same input water and cell design as in the experimental and theoretical program discussed above. The quantity of salt removal can be defined in various ways, such as the decrease in salt concentration of the diluate (averaged over the adsorption step), $\Delta c$, which is the metric we will use in this section. Energy consumption, EC, can be presented in many different ways, such as energy per volume of diluate produced, or energy per unit time. Alternatively, an inverse measure can be used, such as "energy-normalized adsorbed salt" [98]. In this Chapter, we use the metric of energy per amount of salt removed, with unit $\mathrm{kJ} / \mathrm{mol}$.

The calculations are limited to the following conditions: one level of inflow salinity (single salt solution, $\mathrm{NaCl}$ ), either $\mathrm{CC}$ during charge and discharge, or only $\mathrm{CV}$, thus no mixed modes, and pump rate constant (no variation in pump flow rate such as in "stop flow"-mode [99]). For the parameter describing the electronic resistance in cables and current collectors, EER, we use in the calculations a value different from the fit value found for Fig. 4.3, because that value was large compared to work reported in Ref. [162]. Therefore in the present calculations, we set EER to a lower value, in-line with Ref. [162], of EER $=7.0 \Omega \cdot \mathrm{cm}^{2}$.

For CV operation, four parameters can be used to define a cycle: charging volt- 
age, $V_{\mathrm{ch}}$, discharge voltage, $V_{\mathrm{dch}}$, charging time, $t_{\mathrm{ch}}$, and discharge time, $t_{\mathrm{dch}}$. For CC operation, again four parameters define a cycle: endpoint voltage during charging, $V_{\text {up }}$, charging current, $I_{\mathrm{ch}}$, endpoint voltage during discharge, $V_{\text {down }}$, and discharge current, $I_{\mathrm{dch}}$. For both $\mathrm{CV}$ and $\mathrm{CC}$ we discussed in Section 4.3 that, with equal charging and discharge times, water recovery, WR, is not necessarily $50 \%$, and can be different for each calculation. In order to compare CC and $\mathrm{CV}$, one should control the desalination objective, and thus fix WR at a constant value. We make calculations using these four parameters according to the calculation procedure described in Box 4.2.

\section{Box 4.2: calculation procedure}

1. We sweep the first three parameters (for CV: $V_{\mathrm{ch}}, V_{\mathrm{dch}}$, and $t_{\mathrm{ch}}$; for CC: $I_{\text {ch }}, V_{\text {up }}$, and $\left.V_{\text {down }}\right)$ in a large window of suitable values. In Table 4.2 we list for each parameter which domain is scanned, and the interval between each value.

2. Based on the desired WR, we calculate a guess value for the fourth parameter (for $\mathrm{CV}: t_{\mathrm{dch}}$; for $\mathrm{CC}: I_{\mathrm{dch}}$ ). For $\mathrm{CV}$, we use $t_{\mathrm{dch}}=$ $t_{\mathrm{ch}}\left(\frac{1}{\mathrm{WR}_{\text {desired }}}-1\right)$, and for $\mathrm{CC}$, we use $I_{\mathrm{dch}}=\frac{\mathrm{WR}_{\mathrm{desired}} \cdot I_{\mathrm{ch}}}{1-\mathrm{WR}_{\mathrm{desired}}}$, where $\mathrm{WR}_{\text {desired }}$ is the WR we want to obtain.

3. We run for each parameter combination the dynamic model and we make calculations of three desalination cycles (charging and discharge) consequently. Based on the output (current, effluent concentration) of the last cycle, for which we reached a dynamic steady state, we calculate WR, energy consumption (for ER $0 \%$ and ER 50\%) and $\Delta c$.

4. Using a numerical routine, we repeat step 3 with $t_{\mathrm{dch}}(\mathrm{CV})$ or $I_{\mathrm{dch}}(\mathrm{CC})$ as variables to minimize the error given by error = $\left(\mathrm{WR}_{\text {desired }}-\mathrm{WR}_{\text {realized }}\right)^{2}$, where $\mathrm{WR}_{\text {realized }}$ is the $\mathrm{WR}$ calculated by the model.

5 . If the error function approaches zero, thus $\mathrm{WR}_{\text {realized }} \approx \mathrm{WR}_{\text {desired }}$, the calculation was considered successful. For CV, this means that, for given $V_{\mathrm{ch}}, V_{\mathrm{dch}}$, and $t_{\mathrm{ch}}$, we have found a value for $t_{\mathrm{dch}}$ that characterizes a desalination cycle with $\mathrm{WR}_{\text {desired }}$; and for $\mathrm{CC}$, this means that, for given $I_{\mathrm{ch}}, V_{\mathrm{up}}$, and $V_{\text {down }}$, we found a value for $I_{\mathrm{dch}}$. For this desalination cycle, we plot values for energy consumption (for ER 0\% and ER $50 \%$ ) as function of $\Delta c$ in Fig. 4.5. 
6. We repeat this procedure for all combinations of parameters listed in Table 4.2. We note that, for some combinations of parameters, the dynamic model could not sufficiently minimize the error calculated in step 4, and therefore could not find a solution. In those cases, no datapoints were plotted in Fig. 4.5.

Table 4.2: Domains for parameters under study in the calculation procedure. For CC, the parameters are: charging voltage, $V_{\mathrm{ch}}$, discharge voltage, $V_{\mathrm{dch}}$, charging time, $t_{\mathrm{ch}}$, and discharge time, $t_{\mathrm{dch}}$; and for CC: endpoint voltage during charging, $V_{\text {up }}$, charging current, $I_{\mathrm{ch}}$, endpoint voltage during discharge, $V_{\text {down }}$, and discharge current, $I_{\mathrm{dch}}$. For each parameter, a lower boundary (LB), step size (:) and upper boundary (UB) are listed. For some parameters, we use two domains with different step sizes, or we use different domains for each value of WR.

\begin{tabular}{|c|c|c|c|c|c|c|c|}
\hline \multicolumn{4}{|c|}{ Constant voltage } & \multicolumn{4}{|c|}{ Constant current } \\
\hline \multirow[t]{2}{*}{ Parameter } & \multicolumn{3}{|c|}{ Domain } & \multirow[t]{2}{*}{ Parameter } & \multicolumn{3}{|c|}{ Domain } \\
\hline & LB & : & UB & & $\mathrm{LB}$ & : & UB \\
\hline$V_{\mathrm{ch}}(\mathrm{V})$ & 0.2 & 0.2 & 1.2 & $V_{\text {up }}(\mathrm{V})$ & 0.4 & 0.2 & 0.8 \\
\hline \multirow[t]{2}{*}{$V_{\mathrm{dch}}(\mathrm{V})$} & 0.3 & 0.0 & 0.5 & & 0.9 & 0.1 & 1.4 \\
\hline & and & and & & $I_{\mathrm{ch}}$ & 5 & 2.5 & $\begin{array}{l}22.5 \text { (WR } 0.4) \\
27.5 \text { (WR 0.5) } \\
22.5 \text { (WR 0.6) } \\
12.5 \text { (WR 0.7) }\end{array}$ \\
\hline \multirow[t]{2}{*}{$t_{\mathrm{ch}}(\mathrm{s})$} & 60 & 30 & 180 & $V_{\text {down }}(\mathrm{V})$ & 0 & 0.1 & 0.2 \\
\hline & & & & & 0.3 & 0.05 & 0.5 \\
\hline$t_{\mathrm{dch}}(\mathrm{s})$ & calc & cont & WR & $I_{\mathrm{dch}}(\mathrm{A})$ & calc & lated & control WR \\
\hline Constraint & $V_{\mathrm{ch}}$ & & & & & & \\
\hline
\end{tabular}

${ }^{1}$ step 4 of procedure Box 4.2

Fig. 4.5 shows the calculation results for CV and CC for ER 0\% (panel A) and ER $50 \%$ (panel B), where the latter means that $50 \%$ of the energy released during discharge can be reused. The results are shown for WR 50\%. As expected, for any value of $\Delta c$, it is possible to run a cycle in a very wasteful manner, thus at high energy use. However, we are most interested in the lowest values of energy for any given $\Delta c$, i.e., we care about the lower boundary of the calculation results.

We explicitly present the lower boundary in Fig. 4.6. We performed calculations not only for WR 50\%, but also for WR 40, 60 and 70\%, and for each set of calculations we plot the lower boundary. To select the datapoints that mark the boundary, a well-defined procedure is required, which we describe in Box 4.3. 


\section{Box 4.3: selecting datapoints at the lower boundary in an EC- $\Delta c$ plot}

1. We select the datapoint with lowest EC, which will be the starting point marking the lower boundary, with coordinates $\left(x_{\text {start }}, y_{\text {start }}\right)$.

2. Now, the procedure selects the next point - positioned to the right of the starting point - that marks the lower boundary. Therefore, for each datapoint $i$ with $x_{i}>x_{\text {start }}$ the slope is calculated using $a_{i}=\frac{x_{i}-x_{\text {start }}}{y_{i}-y_{\text {start }}}$.

3. The datapoint with the lowest slope is selected as the next point marking the lower boundary.

4. We check whether the point is at the boundary of the calculation domain defined in Table 4.2, or whether it is at one if the inner points. If the latter is the case, we are sure the datapoint is an optimum, which we refer to as an OptLB point. Else, the point is only an optimum considering the boundaries we set for the parameter values. In other words, if the boundaries would have been set differently, probably a datapoint with lower energy consumption would have marked the lower boundary. We refer to this point as a LB point.

5. We repeat this procedure from step 2 onwards, and replace subscript "start" by "prev" with values for coordinates $\left(x_{\mathrm{prev}}, y_{\mathrm{prev}}\right)$ being $\left(x_{i}, y_{i}\right)$ found in step 3 . The procedure is terminated when there is no longer a datapoint for which $x_{i}>x_{\text {prev }}$.

An important assumption behind this procedure is that the starting point has, of all datapoints, the lowest value of $y$, and that the lower boundary one would intuitively draw, increases concavely upward with $x$.

Now, with lower boundaries plotted for different values of WR, with and without $50 \%$ energy recovery, see Fig. 4.6, one can compare CC and CV operational modes. Also, one can select, for a certain value of WR and $\Delta c$, the operational mode and parameter combination that define the cycle with best performance. Comparing CC and CV, Fig. 4.6 shows that, without energy recovery, our procedure predicts not much difference in energy consumption between the two modes. However, with 50\% energy recovery, CC shows a somewhat lower energy consumption than $\mathrm{CV}$.

One may wonder how Fig. 4.6C and D would be with $100 \%$ energy recovery. In fact, calculations with $100 \%$ recovery were made, but resulted in many LB points 

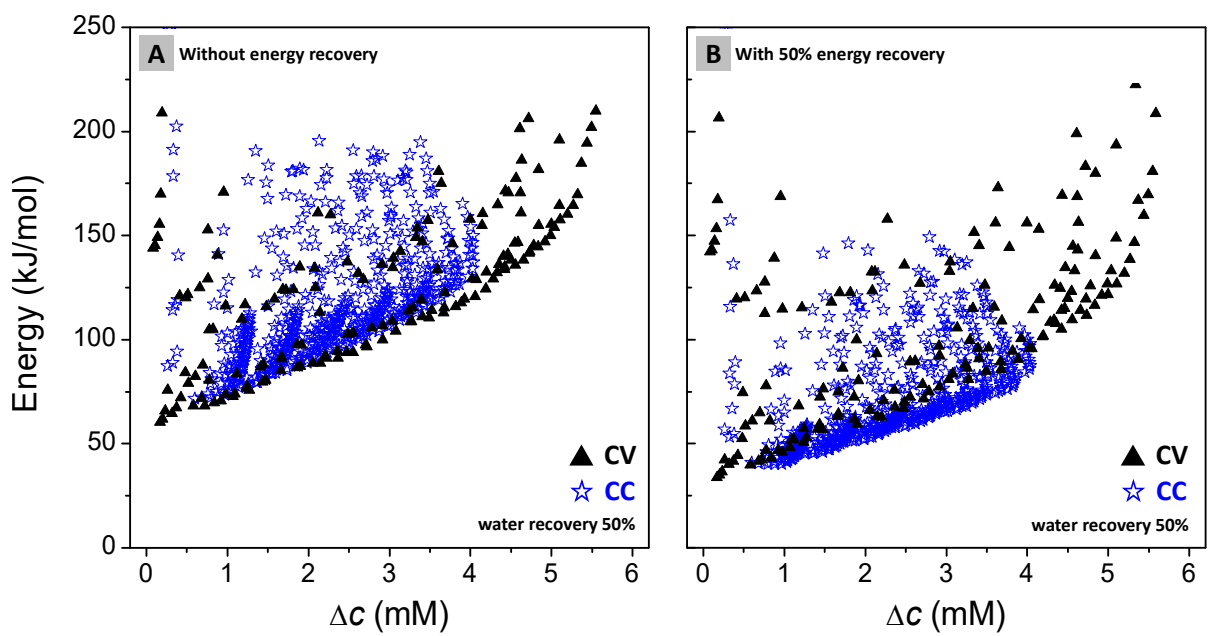

Fig. 4.5: Energy consumption per mol of salt removed as function of desalination, $\Delta c$, A) without and B) with $50 \%$ energy recovery, based on a large sweep of calculated conditions to find the lower boundary for either $\mathrm{CC}$ or $\mathrm{CV}$ operation (water recovery $50 \%$ ).

and only a very limited number of OptLB points. With $100 \%$ recovery the model predicted that it would be most optimum to increase the upper voltage (CC) or the charging and discharge voltages $(\mathrm{CV})$ to high values, in fact to the maximum values we set, as all the input energy could be fully recovered anyway.

Now, we will analyze why, without energy recovery, our procedure predicts not much difference in energy consumption between the two modes, and why, with $50 \%$ energy recovery, CC shows a lower energy consumption than CV. To that end, we split the total energy consumption of two desalination cycles into parts, one for $\mathrm{CV}$ and one for $\mathrm{CC}$, for which $\Delta c$ and WR are the same, and for which we calculated that the energy consumption is at the lower boundary. Conveniently, both for CC and CV, we found an OptLB point for $\Delta c=2.4 \mathrm{mM}$ and $\mathrm{WR}=0.5$, which we select for our analysis. For both operations, we study the energy consumption by charging EDLs (Donnan and Stern potential), ionic resistances in spacer and macropores, and electronic resistances in cables and current collectors. For this energy breakdown analysis, we need an additional set of equations, see Box 4.4. 


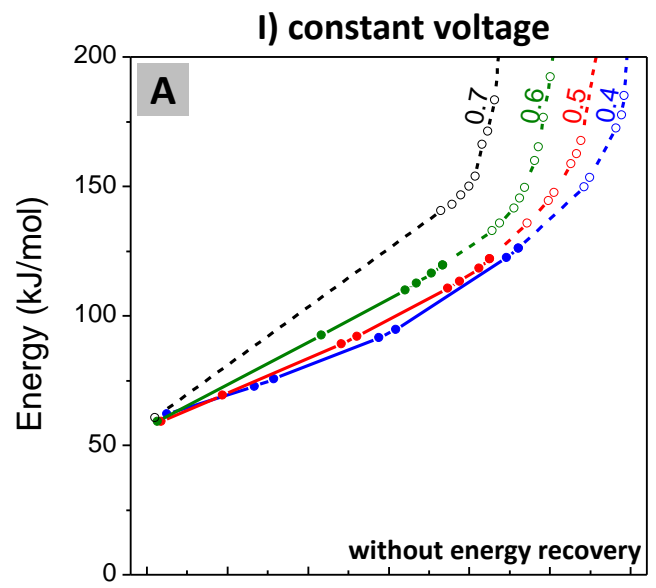

II) constant current
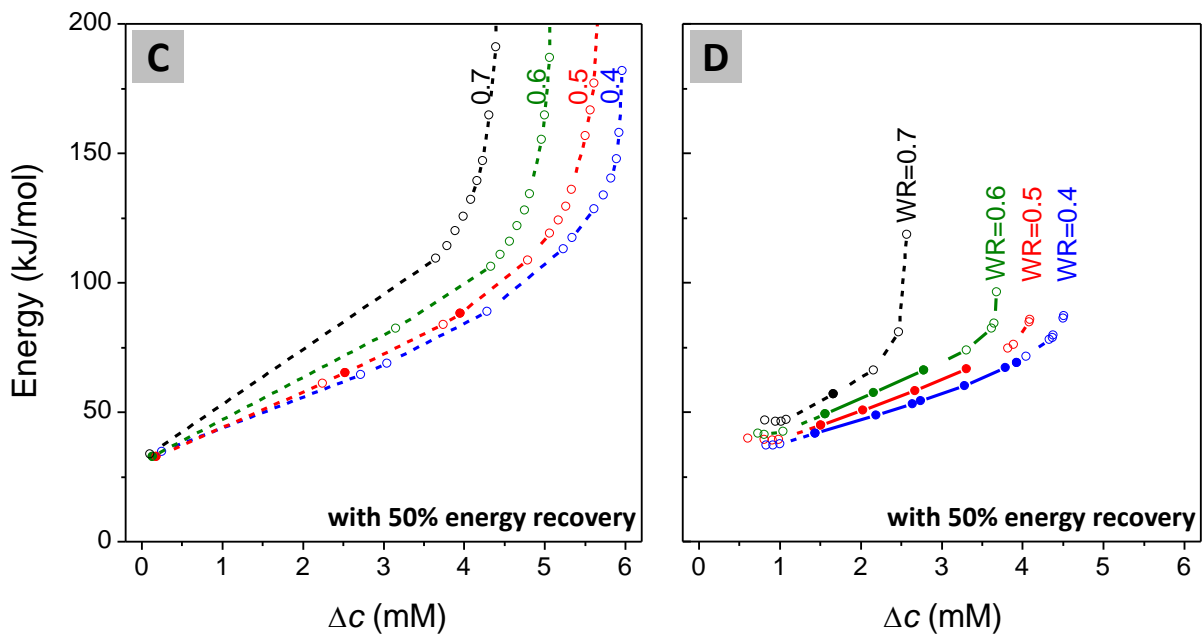

Fig. 4.6: Minimum energy consumption per mol of salt removed as function of desalination, $\Delta c$, and water recovery, WR. Closed datapoints, connected by solid lines, are OptLB points, which indicate optimum values, whereas open datapoints, connected by dashed lines, are LB points, points of lowest energy consumption located at the boundaries of our tested parameter domain. Panel $\mathrm{A}$ and $\mathrm{C}$ show results for constant voltage calculations, Panel B and D for constant current, without (A and B) and with (C and D) $50 \%$ energy recovery. 


\section{Box 4.4: theory for energy breakdown analysis}

\section{Donnan and Stern potential electrodes}

To calculate the energy consumption due to Donnan and Stern potentials in the electrodes, one should consider that values for these potentials and for the ionic current from macropores to micropores (mi) are dependent on time and position in the electrode (in direction from membrane towards current collector). This means that, to calculate the energy consumption, we should multiply the ionic current from $\mathrm{mA}$ to $\mathrm{mi}$ with the potential and integrate over $x$, where $x$ runs from 0 , the membrane-electrode boundary, to $L_{\text {elec }}$, the electrode-current collector boundary, and thereafter over time, $t$. We use

$$
E_{\mathrm{p}}=2 \cdot p_{\mathrm{mi}} \cdot F \cdot V_{\mathrm{T}} \cdot A_{\text {cell }} \cdot \int_{0}^{t} \int_{0}^{L_{\text {elec }}}\left(\frac{\partial \sigma(x, t)}{\partial t} \cdot \Delta \phi_{\mathrm{p}}(x, t)\right) \mathrm{d} x \mathrm{~d} t
$$

where subscript "p" can be replaced by "S" to calculate energy consumption because of the Stern, or by "D", for the Donnan potential. Parameter $p_{\mathrm{mi}}$ is the microporosity, $F$ Faraday's constant $(96,485 \mathrm{C} / \mathrm{mol}), A_{\text {cell }}$ the electrode geometric surface area $\left(\mathrm{m}^{2}\right), \sigma$ the charge density in the micropores $\left(\mathrm{mol} / \mathrm{m}^{3}\right)$ and $V_{\mathrm{T}}$ the thermal voltage, which is given by $V_{\mathrm{T}}=R \cdot T / F$, where $R$ is the gas constant $(8.314 \mathrm{~J} /(\mathrm{mol} \cdot \mathrm{K}))$, and $T$ temperature $(\mathrm{K})$. A factor 2 is included to calculate $E_{\mathrm{p}}$ for both electrodes together, which we also include in Eqs. (4.3) and (4.4).

\section{Ionic resistance electrodes}

To calculate energy consumption due to an ionic resistance in the electrode, we calculate the ionic current in the macropores, which varies across the electrode. Assuming a symmetric cell and an electrolyte with cations and anions that have an equal diffusion coefficient, $D$, the ionic current, $I_{\mathrm{mA}}$, is given by

$$
I_{\mathrm{mA}}(x, t)=-2 \cdot p_{\mathrm{mA}} \cdot F \cdot D \cdot c_{\mathrm{mA}}(x, t) \frac{\partial \phi_{\mathrm{mA}}(x, t)}{\partial x}
$$

where $p_{\mathrm{mA}}$ is the macroporosity, $c_{\mathrm{mA}}$ the concentration of salt in the macropores and $\phi_{\mathrm{mA}}$ the potential in the macropores. The energy consumption due to ionic resistances in the macropores, $E_{\mathrm{mA}}$, can be calculated according to

$$
E_{\mathrm{mA}}=2 \cdot A_{\text {cell }} \cdot V_{\mathrm{T}} \int_{0}^{t} \int_{0}^{L_{\mathrm{elec}}} I_{\mathrm{mA}}(x, t) \cdot \frac{\partial \phi_{\mathrm{mA}}(x, t)}{\partial x} \mathrm{~d} x \mathrm{~d} t .
$$

Please note that this approach to calculate $E_{\mathrm{mA}}$ is different from the one used in Chapter 3 , where we used $E_{\mathrm{mA}}=I^{2} \cdot R_{\mathrm{mA}} \cdot t$, with $I$ the current 
in the system, and $R_{\mathrm{mA}}$ the resistance, for which we calculated a value at the start of a discharge step using Eq. (3.29). Thus, in Chapter 3, $E_{\mathrm{mA}}$ was calculated assuming a constant current and resistance across the macropores, independent of position and time, which is physically not realistic. Using Eq. (4.3), we relax this assumption.

\section{Ionic resistance spacer}

The energy consumption due to ionic resistances in the spacer channel is given by

$$
E_{\mathrm{sp}}=2 \cdot A_{\text {cell }} \cdot V_{\mathrm{T}} \int_{0}^{t} \Delta \phi_{\mathrm{sp}}(t) \cdot I_{\mathrm{A}}(t) \mathrm{d} t
$$

where $I_{\mathrm{A}}(t)$ is the current density $\left(\mathrm{A} / \mathrm{m}^{2}\right)$, and $\Delta \phi_{\mathrm{sp}}$ the potential drop over half the spacer, which is calculated using Eq. (3.14).

\section{Electronic resistance cables and current collectors}

The energy consumption due to electronic resistances in the external electrical circuit (cables, current collectors) is given by

$$
E_{\mathrm{EER}}=\mathrm{EER} \cdot A_{\text {cell }} \int_{0}^{t} I_{\mathrm{A}}(t)^{2} \mathrm{~d} t
$$

where EER is the external electronic resistance in unit $\Omega \mathrm{m}^{2}$.

The energy consumption due to Donnan and Stern potentials in the electrodes, or in other words, the energy consumption by EDL charging, has a positive value during charging and a negative value during discharge, see Fig. 4.7. Clearly, we observe that all energy stored in the Stern layer is released during discharge. For Donnan, however, not all energy stored can be recovered during discharge, which can be explained as follows. During charging, the Donnan layer is charged from low salinity in the macropores, resulting in a high Donnan potential between macropores and micropores, while during discharge, the Donnan layer is discharged with high salt concentration in the macropores, resulting in a lower Donnan potential. Consequently, the energy released from the Donnan layer during discharge is less than the energy required to charge the layer.

Fig. 4.7 also shows that, both during charging and discharge, energy is dissipated due to the ionic resistances in the spacer and electrodes $(\mathrm{mA})$, and due to electronic resistances in cables and current collectors (EER). Comparing CV and $\mathrm{CC}$, we observe that the energy dissipation due to resistances is higher in CV than in CC, both during charging and discharge, which can probably be explained by 


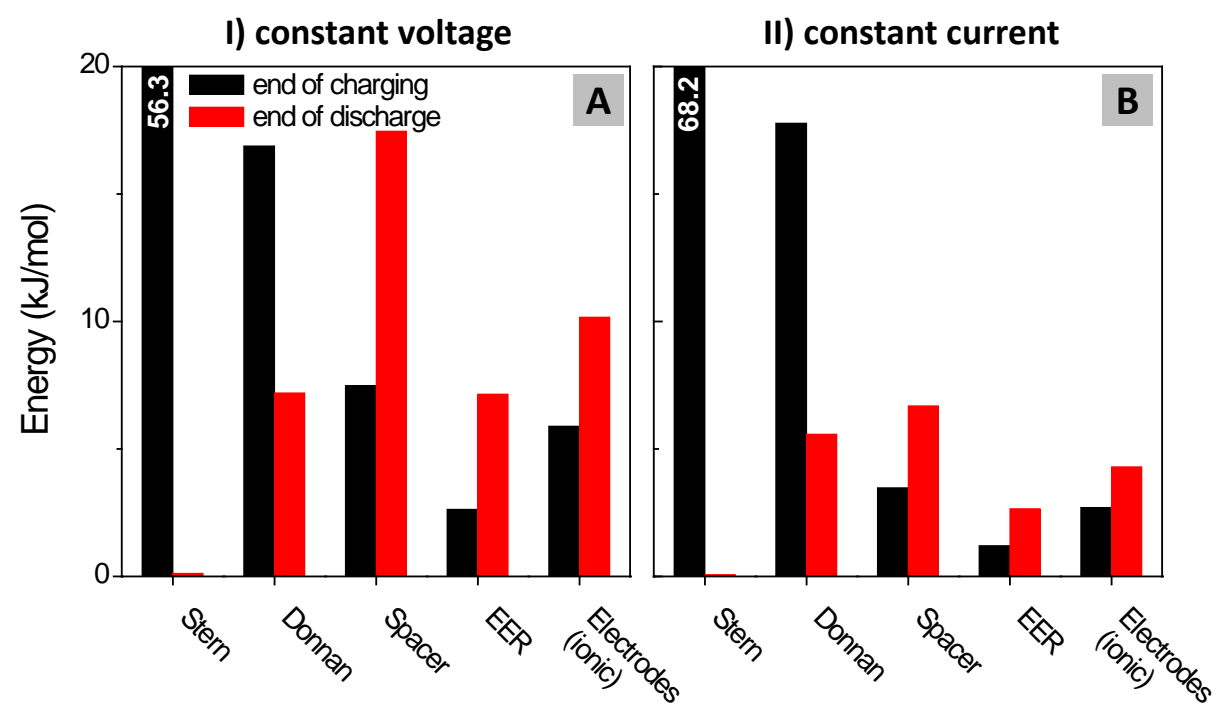

Fig. 4.7: Energy consumption at the end of charging and at the end of discharge of a CDI cell, constant voltage (A) and constant current (B) operation (for both panels: desalination $2.4 \mathrm{mM}$, water recovery $50 \%$ ). Energy consumed by charging EDLs (Donnan and Stern) increases during charging and can (partly) be recovered during discharge. Energy consumed by ionic resistances in spacer and electrodes ( $\mathrm{mA}$ ), and by electronic resistances in cables and current collectors (EER) increases during charging and discharge. The total energy consumption at the end of the charging step for CV is 89.2 and for CC $93.4 \mathrm{~kJ} / \mathrm{mol}$, and at the end of discharge for CV 42.1 and for CC 19.3 $\mathrm{kJ} / \mathrm{mol}$. 
the high currents directly at the start of a CV charging step and, consequently, by the low salt concentrations in the spacer and electrodes, resulting in a high resistance.

Although the energy dissipation due to resistances in $\mathrm{CV}$ mode is much higher than in $\mathrm{CC}$ mode, we find that the total energy consumption at the end of a charging step is slightly lower for CV compared to CC $(89.2$ vs. $93.4 \mathrm{~kJ} / \mathrm{mol})$. This brings us to the conclusion that, for $\mathrm{CV}$, the EDL can be charged less to reach given $\Delta c$ and WR.

\subsection{Conclusions}

In this Chapter, we presented a methodology to compare two operational modes in CDI: constant voltage (CV) and constant current (CC). In this methodology, we used the dynamic CDI model that we outlined in previous work. We defined several constraints and parameters describing desalination cycles for both CC and $\mathrm{CV}$, and we calculated for a sweep of parameter combinations the energy consumption (with and without 50\% energy recovery during discharge), water recovery, WR, and the decrease in salt concentration of the diluate (averaged over the charging step), $\Delta c$. Thereafter, we plotted the lowest values of energy as function of $\Delta c$ and WR.

This robust methodology showed that, in our example calculation, without energy recovery, the lowest values of energy consumption of CV and CC operation are approximately the same. If $50 \%$ of the energy released during discharge can be recovered and reused in CDI, CC has a somewhat lower energy consumption than CV.

We analyzed for which parameter combinations we find the lowest energy consumption for desalination, given $\Delta c$ and $\mathrm{WR}$, both for $\mathrm{CC}$ and $\mathrm{CV}$. For future work, one could analyze mixed modes of operation (e.g. CC charging and CV discharge, or vice-versa), and operation with a lower flow rate during discharge than during charging, an operational mode that can be used to increase WR. Furthermore, one could use our methodology to find parameter combinations that result in optimum desalination performance for membrane-CDI systems, and other cell designs. 


\section{Appendix}

\section{A Experimental design for model validation}

Our experimental program is based on the CDI stack described in detail in Chapter 2, consisting of four cells, each cell containing two porous carbon electrodes of $\sim 33.8 \mathrm{~cm}^{2}$ area (dry mass of $0.552 \mathrm{~g}$ per electrode) and a thickness of $\sim 250$ $\mu \mathrm{m}$. These electrodes (Materials \& Methods, PACMM ${ }^{\mathrm{TM}} 203$, Irvine, CA, USA) are characterized in Ref. [71]. A spacer (Glass fibre prefilter, Millipore) of 316 $\mu \mathrm{m}$ thickness keeps the electrodes apart and allows for fluid flow (compressed thickness, see Ref. [162]). The water flow rate per cell is $7.5 \mathrm{~mL} / \mathrm{min}$ and the inflow salt concentration $(\mathrm{NaCl})$ is $20 \mathrm{mM}$. The salt solution is pumped from a vessel with a volume of $10 \mathrm{~L}$, through the cell, thereafter passes a conductivity sensor that records a value each second, and recirculates back to the vessel. The solution in the vessel is purged with $\mathrm{N}_{2}$ gas to minimize the concentration of dissolved oxygen in solution.

Typical results of the experiments are presented in Fig. 4.A.1 for the salt effluent concentration, current (in CV mode) and cell voltage (in CC mode) for three consecutive cycles in each case. From these data we calculate the salt removal per cycle (in $\mathrm{mg} \mathrm{NaCl}$ per gram of both electrodes) and the charge transferred in a cycle (in $\mathrm{C} / \mathrm{g}$ ), see Fig. 4.A.2. For CC operation, we do not present data for charge (as they are simply equal to current multiplied by the half cycle time), but present results in Fig. 4.A.2D of the average voltage during charging and discharge. With all these data at hand, we can calculate the electric energy input during charging by integrating the product of cell voltage and current over the duration of the charging step. This is the energy input without energy recovery. We also calculate the energy released during discharge (again from integrating 
the product of current and cell voltage) and subtract this full amount from the input energy. This gives us the data for overall energy consumption assuming $100 \%$ recovery. Energy is given per mole of salt removed in $\mathrm{kJ} / \mathrm{mol}$. A numerical value expressed in $\mathrm{kJ} / \mathrm{mol}$, can be divided by five to obtain an approximate value for energy consumption in the unit of "kT per ion". Thus a value of 100 $\mathrm{kJ}$ per mol salt removal corresponds to $20 \mathrm{kT}$ energy use per ion removed. To calculate desalination, $\Delta c$, we average the difference between inflow and effluent concentration of the CDI stack over the duration of an adsorption step. 

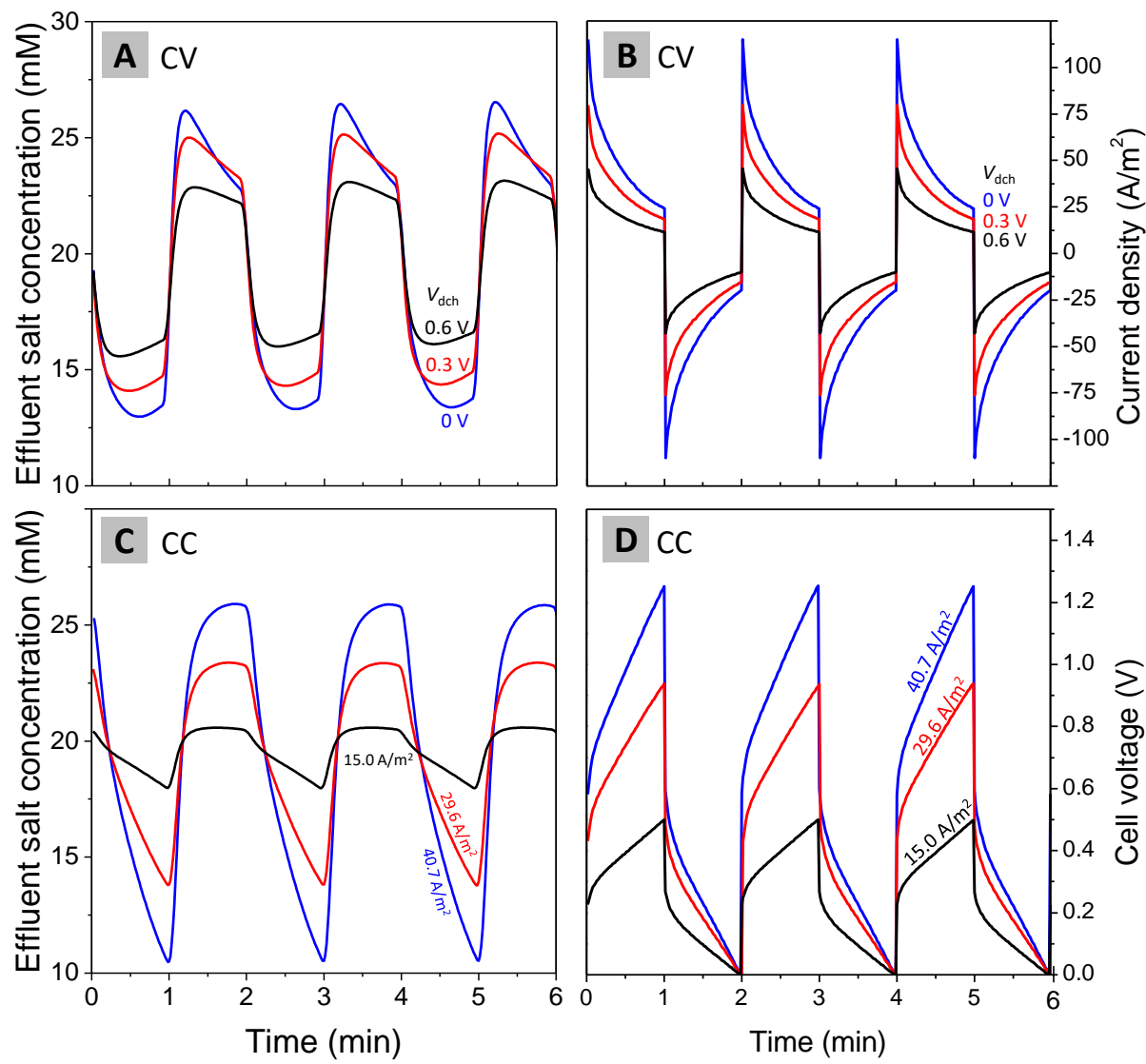

Fig. 4.A.1: Experimental results for CDI cycles operated in $\mathrm{CV}$ or $\mathrm{CC}$ mode with equal duration of charging and discharge. Effluent salt concentration is shown for A) $\mathrm{CV}$ and $\mathrm{C}$ ) CC. For CV, B) shows current density (given by current per cell in A, divided by $33.8 \mathrm{~cm}^{2}$ electrode surface area), and for CC, D) shows discharge voltage, $V_{\mathrm{dch}}=1.0 \mathrm{~V}$. In $\mathrm{CV}$ mode the charging voltage is $V_{\mathrm{ch}}=1.0 \mathrm{~V}$ (duration of full cycle, $\mathrm{CT}$, is $2 \mathrm{~min}$ in all cases). 

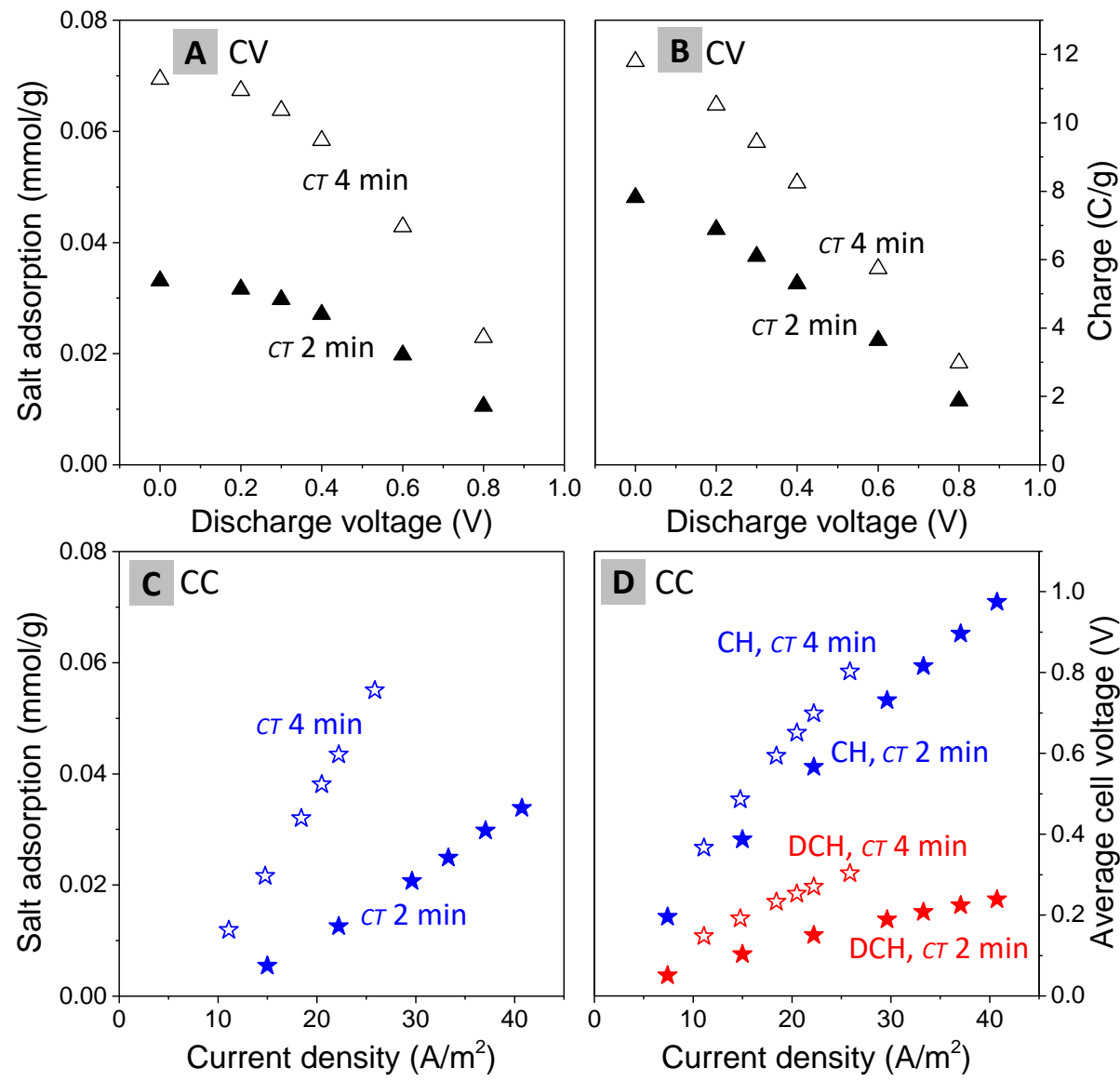

Fig. 4.A.2: Experimental results for CDI cycles operated in CV or CC mode. For CV, A) salt adsorption and B) charge are reported as function of the discharge voltage. For $\mathrm{CC}, \mathrm{C})$ salt adsorption and average cell voltage during charging $(\mathrm{CH})$ and discharge $(\mathrm{DCH})$ are reported as function of current density. The duration of a full cycle, CT, is either 2 min or 4 min. 


\section{Dynamics of ion adsorption from mixtures of salts in CDI}

This Chapter has been published as:

J.E. Dykstra, J. Dijkstra, A. van der Wal, H.V.M. Hamelers, S. Porada, On-line method to study dynamics of ion adsorption from mixtures of salts in capacitive deionization, Desalination 390 (2016) 47-52. 


\section{Abstract}

Capacitive Deionization (CDI) is a water desalination technology that adsorbs ions into two electrically oppositely charged porous electrodes. Here, we introduce a novel method to analyze the effluent concentration of a salt solution with multiple ionic species by directing the outflow of a CDI cell to an inductively coupled plasma optical emission spectroscopy (ICP-OES) instrument. Compared to previous methods based on manual sampling, the on-line use of ICP-OES allows collecting more accurate time-dependent ion adsorption data, and therefore, ion dynamics can be studied even at very short cycle times. We use this method to study ion adsorption from a mixed solution containing two monovalent cations with similar radius, namely $\mathrm{K}^{+}$and $\mathrm{Na}^{+}$. We find that $\mathrm{K}^{+}$ions are preferentially adsorbed over sodium ions, due to their higher mobility. Furthermore, we compare our experimental findings with a novel multicomponent electromigration model that calculates dynamic adsorption of ions from solutions of multiple salts. Whereas we find good agreement between data and theory at short cycle times, we observe a considerable discrepancy for longer cycles. 


\subsection{Introduction}

Capacitive Deionization(CDI) is a desalination technology employing porous electrodes [2]. By applying a voltage between two electrodes, cations are adsorbed into the cathode, while anions are adsorbed into the anode. The ions are stored in the micropores of the porous carbon electrodes, and water flowing through the spacer channel is desalinated (adsorption or charging step). After the electrodes are saturated, the ions are released by short circuiting the electrodes, resulting in a concentrated effluent stream (desorption or discharge step).

Recent research interests in the field of CDI include synthesis of new electrode materials and their chemical modification [11, 33, 51, 88-90], application of ion exchange membranes [27, 34, 162], and the design of novel CDI cells with, for example, wire-shaped electrodes [80] or flow electrodes [5, 72, 73].

Another research interest is the application of CDI for ion selective removal, i.e., removing only certain ions, but keeping other ions in solution. Recently, it has been shown that time-dependent ion selective removal can be achieved in CDI [47]. In this study it was found that, after applying a sufficiently high charging voltage, first monovalent $\mathrm{Na}^{+}$ions are preferentially adsorbed into the electrical double layers (EDLs). After some time, however, divalent $\mathrm{Ca}^{2+}$ ions gradually replace $\mathrm{Na}^{+}$ions in the EDLs. Another study investigated selective adsorption of $\mathrm{Ca}^{2+}$ with a composite electrode of carbon nanotubes and Caselective zeolite [94]. Other studies have shown selective adsorption of $\mathrm{NO}_{3}{ }^{-}$ions using ion selective exchange resins [95], or non-specific ion exchange membranes [28]. Lastly, Hou et al. [96] investigated selective adsorption of ions with activated carbon electrodes in CDI, and showed selective removal due to differences in radius and charge of ionic species.

In present Chapter, we study time-dependent adsorption of $\mathrm{K}^{+}$and $\mathrm{Na}^{+}$ions from a solution of $\mathrm{NaCl}$ and $\mathrm{KCl}$ in $\mathrm{CDI}$. This study is of particular interest, because $\mathrm{K}^{+}$and $\mathrm{Na}^{+}$are both monovalent ions, and are fully dissociated at sufficiently low concentration. Therefore, at equilibrium, one ion is not preferentially adsorbed over the other, according to classical Donnan theory, see Chapter 2.

To study time-dependent ion adsorption, we use an inductively coupled plasma optical emission spectroscopy (ICP-OES) instrument, which is directly, thus online, connected to the outflow of the CDI cell, see Fig. 5.1. Hence, we can measure the effluent concentration of different cations with a sampling interval time varying from 2 to $4 \mathrm{~s}$. Compared with methods described in previous work [47, 163-166], which required manual sampling of the effluent, this interval time is very short, and we can collect more accurate time-dependent salt adsorption data. Also, we can neglect concentration-averaging effects that occur when collecting samples manually (which requires a non-negligible sampling volume). 


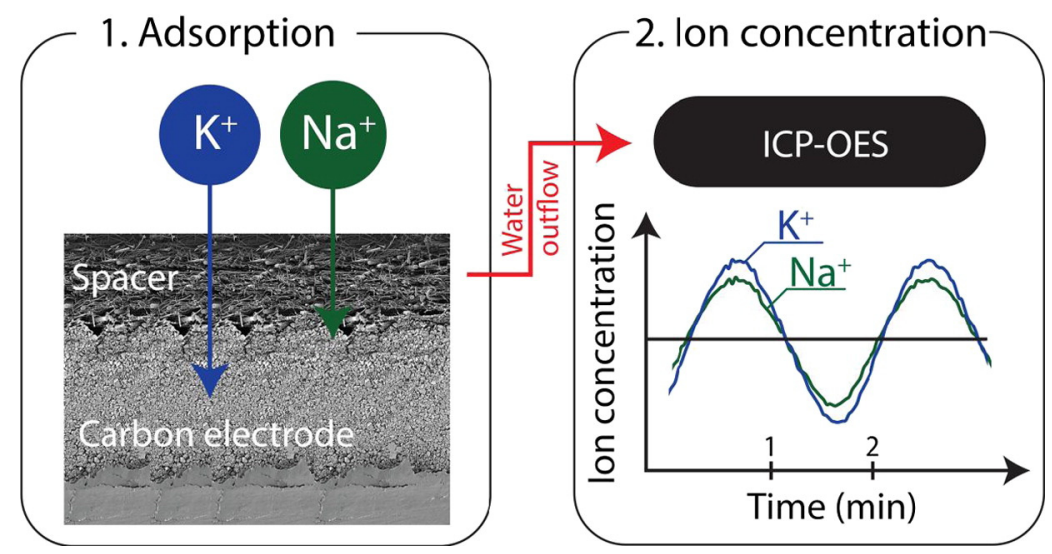

Fig. 5.1: Schematic view of the method used to study time-dependent ion adsorption in Capacitive Deionization. The outflow of the CDI cell is directly connected to the online ICP-OES instrument in order tomeasure the concentration of each ion as function of time.

Therefore, with on-line ICP-OES, time-dependent ion adsorption can be studied, even for very short adsorption and desorption steps. Thus, on-line ICP-OES is a very advantageous method, which has, to the best of our knowledge, not been introduced in CDI research before.

We show that, although $\mathrm{K}^{+}$and $\mathrm{Na}^{+}$ions have the same valence, and a very similar hydrated radius (3.31 and $3.58 \AA$ respectively [167], selective removal of potassium over sodium is possible, due to their different diffusion coefficients $\left(1.90 \cdot 10^{-9}\right.$ and $1.33 \cdot 10^{-9} \mathrm{~m}^{2} / \mathrm{s}$ respectively). Furthermore, we present theoretical results calculated using a novel multicomponent dynamic model, which calculates electromigration of ions in porous carbon electrodes combined with ion adsorption in EDLs. Compared with Chapters 2 and 3, this model is extended to include multiple monovalent ionic species with different diffusion coefficients.

\subsection{Theory}

In our dynamic model, which calculates ion adsorption in porous carbon electrodes, we distinguish two types of pores in the electrodes: micropores and macropores. In the micropores, EDLs are formed, and consequently, ions are adsorbed. In the macropores, ions are transported from the spacer channel to the micropores. Please note that we make a simplification compared to the theory presented in Chapters 2 and 3: we do not consider mesopores in our model. We make this simplification as we do not aim to fit model parameters to experimen- 
tal data; we will only use the model to describe observed trends in experimental data.

To model dynamic adsorption of ions in CDI, we couple two different models. Firstly, we use the improved modified Donnan model (i-mD model), which is an equilibrium model to relate voltage, charge density and ion adsorption to one another, see Chapter 2. Secondly, we use a dynamic transport model, which describes ion transport in the macropores. In this Chapter, we will only present the equations used in the transport model. For a detailed description of the i-mD model, see Chapter 2.

The transport model describes transport of ions in both electrodes, and considers that each ionic species has a different diffusion coefficient. The model assumes that all ions are monovalent, and only considers capacitive processes, i.e., we exclude possible Faradaic (redox) reactions. We neglect tortuosity effects.

The molar flux of ions through the macropores in the electrodes is described by the Nernst-Planck equation,

$$
J_{i}=-D_{i}\left(\frac{\partial c_{\mathrm{mA}, i}}{\partial x}+z_{i} \cdot c_{\mathrm{mA}, i} \cdot \frac{\partial \phi_{\mathrm{mA}}}{\partial x}\right)
$$

where subscript $i$ refers to ion species $i$, and where $J_{i}$ is the molar ion flux in $\mathrm{mol} / \mathrm{m}^{2} / \mathrm{s}, D_{i}$ the ion diffusion coefficient in $\mathrm{m}^{2} / \mathrm{s}, c_{\mathrm{mA}, i}$ the ion concentration in the macropores in $\mathrm{mol} / \mathrm{m}^{3}, z_{i}$ the ion valency, $\phi_{\mathrm{mA}}$ the dimensionless potential in the macropores, and $x$ the coordinate running from the spacer-electrode boundary to the electrode-current collector boundary.

At each position in the electrode, we evaluate for each ionic species the mass balance

$$
\frac{\partial}{\partial t}\left(p_{\mathrm{mA}} \cdot c_{\mathrm{mA}, i}+p_{m i} \cdot c_{\mathrm{mi}, i}\right)=-p_{\mathrm{mA}} \cdot \frac{\partial J_{i}}{\partial x}
$$

where $p_{\mathrm{mA}}$ and $p_{\mathrm{mi}}$ are the macro-, and microporosity of the electrode, and where $c_{\mathrm{mi}, i}$ is the ion concentration in the micropores.

Furthermore, we need the electroneutrality condition for the macropores

$$
\sum_{i} z_{i} \cdot c_{\mathrm{mA}, i}=0
$$

where $i$ runs over all ions in the system, i.e. $\mathrm{Na}^{+}, \mathrm{K}^{+}$, and $\mathrm{Cl}^{-}$.

The micropore charge density, $\sigma_{\mathrm{mi}}$, and total micropore ion concentration, $c_{\text {ions,mi }}$ are defined as

$$
\begin{aligned}
\sigma_{\mathrm{mi}} & =\sum_{i} z_{i} \cdot c_{\mathrm{mi}, i} \\
c_{\mathrm{ions}, \mathrm{mi}} & =\sum_{i} c_{\mathrm{mi}, i} .
\end{aligned}
$$

Based on Eqs. (5.1)-(5.4), we set up a system of three balance equations, describing the evolution of $\sigma_{\mathrm{mi}}, c_{\mathrm{mA}, \mathrm{T}}$, which is the total concentration of cations 
or anions in the macropores, and the concentration of one of the cations, either $\mathrm{Na}^{+}$or $\mathrm{K}^{+}$.

The first balance equation evaluates $\sigma_{\mathrm{mi}}$ over time and is given by

$$
p_{\mathrm{mi}} \cdot \frac{\partial \sigma_{\mathrm{mi}}}{\partial t}=-p_{\mathrm{mA}} \sum_{i} z_{i} \frac{\partial J_{i}}{\partial x} .
$$

The second balance equation evaluates $c_{\mathrm{mA}, \mathrm{T}}$ over time and is given by

$$
\frac{\partial}{\partial t}\left(2 \cdot p_{\mathrm{mA}} \cdot c_{\mathrm{mA}, \mathrm{T}}+p_{\mathrm{mi}} \cdot c_{\mathrm{ions}, \mathrm{mi}}\right)=-p_{\mathrm{mA}} \sum_{i} \frac{\partial J_{i}}{\partial x} .
$$

Thirdly, we include Eq. (5.2) for one of the cations, either for $\mathrm{Na}^{+}$or $\mathrm{K}^{+}$. Note that the choice between $\mathrm{Na}^{+}$or $\mathrm{K}^{+}$is arbitrary, and the same output is obtained, irrespective of the choice. In general, in case we model a system with an electrolyte consisting of multiple monovalent ionic species, Eq. (5.2) has to be included for $N_{\mathrm{c}}-1$ cationic species, and for $N_{\mathrm{a}}-1$ anionic species, where $N_{\mathrm{c}}$ is the number of cationic species and $N_{\mathrm{a}}$ the number of anionic species.

Across the electrode, at each position, $\phi_{\mathrm{mA}}$ is related to the potential in the carbon matrix, $\phi_{\mathrm{e}}$, where subscript "e" can be replaced by "c" for the cathode, or "a" for the anode, and is related to the Donnan potential, $\Delta \phi_{\mathrm{D}}$, and the Stern potential, $\Delta \phi_{\mathrm{S}}$. These potentials follow from the i-mD model, see Chapter 2.

In this Chapter, we assume there is no electronic resistance across the electrode, which is supported by experimental work presented in Box 3.1, and to simplify our model even further, we assume there is no electronic resistance in cables and current collectors, which connect the electrodes with the power source. To that end, we can relate the cell voltage, $V_{\text {cell }}$, to a position invariant value of the electrode potential, $\phi_{\mathrm{c}}$ for the cathode, or $\phi_{\mathrm{a}}$ for the anode, given by

$$
V_{\text {cell }}=V_{\mathrm{T}} \cdot\left(\phi_{\mathrm{c}}-\phi_{\mathrm{a}}\right)
$$

The thermal voltage, $V_{\mathrm{T}}$, is given by $V_{\mathrm{T}}=R T / F$, where $R$ is the gas constant $(8.314 \mathrm{~J} /(\mathrm{mol} \mathrm{K})), T$ the temperature $(\mathrm{K})$ and $F$ Faraday's constant $(96,485$ $\mathrm{C} / \mathrm{mol})$.

To evaluate the ion concentrations in the spacer channel, we set up a spacer mass balance equation for each ionic species, except for one, which can be arbitrary chosen. Thus, for $N_{\mathrm{c}}+N_{\mathrm{a}}-1$ ions, we set up a mass balance equation according to

$$
p_{\mathrm{sp}} \frac{\partial c_{\mathrm{sp}, i}}{\partial t}=-\frac{p_{\mathrm{mA}}}{L_{\mathrm{sp}}} \sum_{e=A, C} J_{e, i}+\frac{\Phi_{\mathrm{v}}}{L_{\mathrm{sp}} \cdot A_{\text {cell }}}\left(c_{\text {inflow }, i}-c_{\mathrm{sp}, i}\right)
$$

where $c_{\mathrm{sp}, i}$ is the ion concentration in the spacer channel, $\Phi_{\mathrm{v}}$ the flow rate through the cell, $L_{\mathrm{sp}}$ the thickness of the spacer channel, $A_{\text {cell }}$ the electrode geometric 
surface area and $c_{\text {inflow, } i}$ the inflow ion concentration in the spacer channel, i.e., the ion concentration in the feed water. The molar flux of ions from the spacer channel into the anode or cathode, $J_{e, i}$, where subscript $e$ can be "a" for the anode or "c" for the cathode, is given by Eq. (5.1). Note that Eq. (5.8) is different from the spacer balance equation, Eq. (11), in Chapter 3, as we do not model concentration gradients in the direction perpendicular to the flow, neither we describe the development of concentration profiles between the out- and inflow of the cell. Furthermore, in the spacer channel, the electroneutrality condition holds, Eq. (5.3), where subscript "mA" is replaced by "sp".

The potential drop over the spacer channel, $\Delta \phi_{\mathrm{sp}}$, is evaluated as follows

$$
\Delta \phi_{\mathrm{sp}}=L_{\mathrm{sp}} \frac{I_{\mathrm{A}}}{F \sum_{i} z_{i}^{2} \cdot D_{i} \cdot c_{i}}
$$

where $L_{\mathrm{sp}}$ is the thickness of the spacer channel and $I_{\mathrm{A}}$ is the current density in $\mathrm{A} / \mathrm{m}^{2}$. The potential drop over the spacer, $\Delta \phi_{\mathrm{sp}}$, is related to the carbon matrix potential difference between the anode $\left(\phi_{\mathrm{a}}\right)$ and cathode $\left(\phi_{\mathrm{c}}\right)$, according to

$$
\Delta \phi_{\mathrm{sp}}=\phi_{\mathrm{a}}-\phi_{\mathrm{c}}
$$

On the spacer-electrode boundary, the concentration $c_{\mathrm{sp}, i}$ of each ion is equal to the concentration in the macropores at the boundary.

The current density is evaluated on the spacer-electrode boundary as follows

$$
I_{\mathrm{A}}=F \cdot \sum_{i} z_{i} \cdot J_{e, i}
$$

On the electrode-current collector boundaries (i.e., the "backside" of the electrode), we apply the boundary conditions

$$
\left.\frac{\partial c_{\mathrm{mA}, i}}{\partial x}\right|_{\mathrm{E} / \mathrm{C}}=0,\left.\frac{\partial \phi_{\mathrm{mA}}}{\partial x}\right|_{\mathrm{E} / \mathrm{C}}=0
$$

where the first equation holds for each ion.

\subsection{Materials and methods}

All experiments were performed using a CDI stack which contained two parallel cells. Each cell consisted of two porous carbon electrodes (Materials \& Methods, $\mathrm{PACM}{ }^{\mathrm{TM}} 203$, Irvine, $\mathrm{CA}, \delta_{\mathrm{e}} \sim 250 \mu \mathrm{m}, \mathrm{m}_{\text {tot }}=2.2 \mathrm{~g}$ total mass per stack), which were connected with graphite current collectors (thickness $\delta \sim 250$ $\mu \mathrm{m})$ to the electrical circuit. A polymer spacer (Glass fibre prefilter, Millipore, compressed thickness of $\delta_{\mathrm{sp}} \sim 250 \mu \mathrm{m}$ ) was placed between the electrodes. An aqueous solution with an inflow composition of $5 \mathrm{mM} \mathrm{NaCl}$ and $5 \mathrm{mM} \mathrm{KCl}$ flowed 
through the spacers at a constant flow rate of $7.5 \mathrm{~mL} / \mathrm{min} /$ cell. The charging voltage was applied with a potentiostat that also measured the current between cathode and anode. The current was integrated over time, for the duration of the adsorption step, resulting in the total charge, which was equal (after correcting for a small leakage charge) to the charge released again in the desorption step. This total charge with dimension Coulomb was divided by the total electrode mass, $\mathrm{m}_{\text {tot }}$, and by Faraday's number, to obtain the charge expressed in mol/g. To measure the effluent ion concentrations, the outflow of the CDI cell was connected to the peristaltic pump of the ICP-OES instrument (Optima 5300 DV, Perkin Elmer) using a T-piece and polyethylene tubing with an internal diameter of $1.0 \mathrm{~mm}$, which was positioned $\sim 5 \mathrm{~cm}$ behind the outflow of the stack. The peristaltic pump was operated at a constant flow rate of $0.8 \mathrm{~mL} / \mathrm{min}$. Just before entering the nebulizer, the liquid flow from the CDI stack was mixed with a solution of $5 \mathrm{ppm} \mathrm{Y}$ in $2 \%$ nitric acid. This setup could continuously correct variations in the liquid flow entering the nebulizer that may be caused by the peristaltic pump (temperature, wear). A quartz cyclonic spray chamber and a perfluoroalkoxy (PFA) MicroFlow nebulizer (Elemental Scientific, Perkin Elmer) were used to inject the liquid flow into the ICP-OES instrument, with $\operatorname{Ar}(99.9990 \%$ mole, Linde Gas) as nebulizing gas. The ICP-OES instrument was operated in radial viewing mode to simultaneously detect $\mathrm{Ar}, \mathrm{Y}, \mathrm{K}$ and $\mathrm{Na}$ at a wavelength of $420.069 \mathrm{~nm}, 371.029 \mathrm{~nm}, 766.490 \mathrm{~nm}$ and $589.592 \mathrm{~nm}$, respectively. Instrument gas flow settings were $15 \mathrm{~mL} / \mathrm{min}$ for the plasma, $0.2 \mathrm{~mL} / \mathrm{min}$ for the auxiliary and $0.65 \mathrm{~mL} / \mathrm{min}$ for the nebulizer. The power of the radio frequency generator was set to $1400 \mathrm{~W}$. Plasma read times were varied between 2 and $4 \mathrm{~s}$ to obtain the optimal number of data points in each experiment. Seven calibration standards were prepared in ultrapure water. Concentrations of the standards were $0,1,2,4,6,8$ and $10 \mathrm{mM}$ of sodium and potassium, respectively. The calibration curve was recorded prior to each experiment, using three consecutive replicates for all standards. A least-squares regression line was used to describe the linear relationship between the concentration of the standards and the intensity of the measured signals at both wavelengths. The ion effluent concentration was integrated over time, for the duration of the adsorption step, resulting in the total ion adsorption, which was equal (within measurements errors) to the amount of ions released in the desorption step. This total ion adsorption was divided by the total electrode mass, $\mathrm{m}_{\text {tot }}$, and multiplied by the molar mass of the ion (see Table 5.1), $\mathrm{M}_{\mathrm{w}}$, to obtain the ion adsorption expressed in $\mathrm{mg} / \mathrm{g}$. 
Table 5.1: System and electrode dimensions, operational parameters, and settings for theoretical calculations.

\begin{tabular}{|c|c|c|c|}
\hline \multicolumn{4}{|c|}{ Experimental parameters } \\
\hline$L_{\text {elec }}$ & Electrode thickness & 316 & $\mu \mathrm{m}$ \\
\hline$A_{\text {cell }}$ & Electrode geometric surface area & 33.8 & $\mathrm{~cm}^{2}$ \\
\hline$\Phi_{\mathrm{v}}$ & Flow rate through the cell & 7.5 & $\mathrm{~mL} / \mathrm{min}$ \\
\hline$N_{\mathrm{s}}$ & Number of cells in the CDI stack & 2 & \\
\hline$T$ & Temperature & 298 & K \\
\hline$\rho_{\text {elec }}$ & Electrode mass density & 0.55 & $\mathrm{~g} / \mathrm{mL}$ \\
\hline \multicolumn{4}{|c|}{ Molar mass of ionic species } \\
\hline$M_{\mathrm{w}, \mathrm{Na}}{ }^{+}$ & Molar mass of $\mathrm{Na}^{+}$ & 22.9 & $\mathrm{~g} / \mathrm{mol}$ \\
\hline$M_{\mathrm{w}, \mathrm{K}}+$ & Molar mass of $\mathrm{K}^{+}$ & 39.1 & $\mathrm{~g} / \mathrm{mol}$ \\
\hline$M_{\mathrm{w}, \mathrm{Cl}{ }^{-}}$ & Molar mass of $\mathrm{Cl}^{-}$ & 35.5 & $\mathrm{~g} / \mathrm{mol}$ \\
\hline \multicolumn{4}{|c|}{ Values used in the i-mD model } \\
\hline$E$ & Micropore ion-correlation energy & 300 & $\mathrm{kT} \mathrm{mol} \mathrm{m}{ }^{-3}$ \\
\hline$C_{\mathrm{S}}$ & Stern capacitance in the zero-charge limit & 100 & $\mathrm{~F} / \mathrm{mL}$ \\
\hline$\alpha$ & Charge dependence of Stern capacitance & 30 & $\mathrm{~F} \mathrm{~m} \mathrm{~mol}^{-2}$ \\
\hline \multicolumn{4}{|c|}{ Spacer channel properties } \\
\hline$L_{\mathrm{sp}}$ & Spacer channel thickness (when compressed) & 316 & $\mu \mathrm{m}$ \\
\hline$p_{\mathrm{sp}}$ & Spacer channel porosity (when compressed) & 0.708 & \\
\hline \multicolumn{4}{|c|}{ Values used in the transport model } \\
\hline$D_{\mathrm{Na}^{+}}$ & Diffusion coefficient of $\mathrm{Na}^{+}$ & 1.33 & $\cdot 10^{-9} \mathrm{~m}^{2} / \mathrm{s}$ \\
\hline$D_{\mathrm{K}^{+}}$ & Diffusion coefficient of $\mathrm{K}^{+}$ & 1.90 & $\cdot 10^{-9} \mathrm{~m}^{2} / \mathrm{s}$ \\
\hline$D_{\mathrm{Cl}^{-}}$ & Diffusion coefficient of $\mathrm{Cl}^{-}$ & 2.02 & $\cdot 10^{-9} \mathrm{~m}^{2} / \mathrm{s}$ \\
\hline$p_{\mathrm{mA}}$ & Macroporosity & 0.416 & \\
\hline$p_{\mathrm{mi}}$ & Microporosity & 0.3 & \\
\hline$p_{\mathrm{sk}}$ & Fraction skeleton material of electrode & 0.29 & \\
\hline
\end{tabular}

\subsection{Results and discussion}

We conducted CDI experiments at different cycle times (CTs), i.e., at different durations the desalination cycle, comprising both adsorption and desorption step. Note that, in present Chapter, the duration of the adsorption step is always equal to the desorption step. During the adsorption step, when we apply a charging voltage, $V_{\mathrm{ch}}$, of $1.0 \mathrm{~V}$, we observe that the effluent concentration of $\mathrm{K}^{+}$decreases faster and to lower values than the concentration of $\mathrm{Na}^{+}$, both for the experiments with a short CT (4 min., Fig. 5.2A) and for a long CT (6 h, Fig. 5.2B). During the desorption phase, when the electrodes are short-circuited, we see that $\mathrm{K}^{+}$ions are released faster and the $\mathrm{K}^{+}$effluent concentration reaches higher values than $\mathrm{Na}^{+}$. The data presented in Fig. 5.2 shows that the described on-line method 

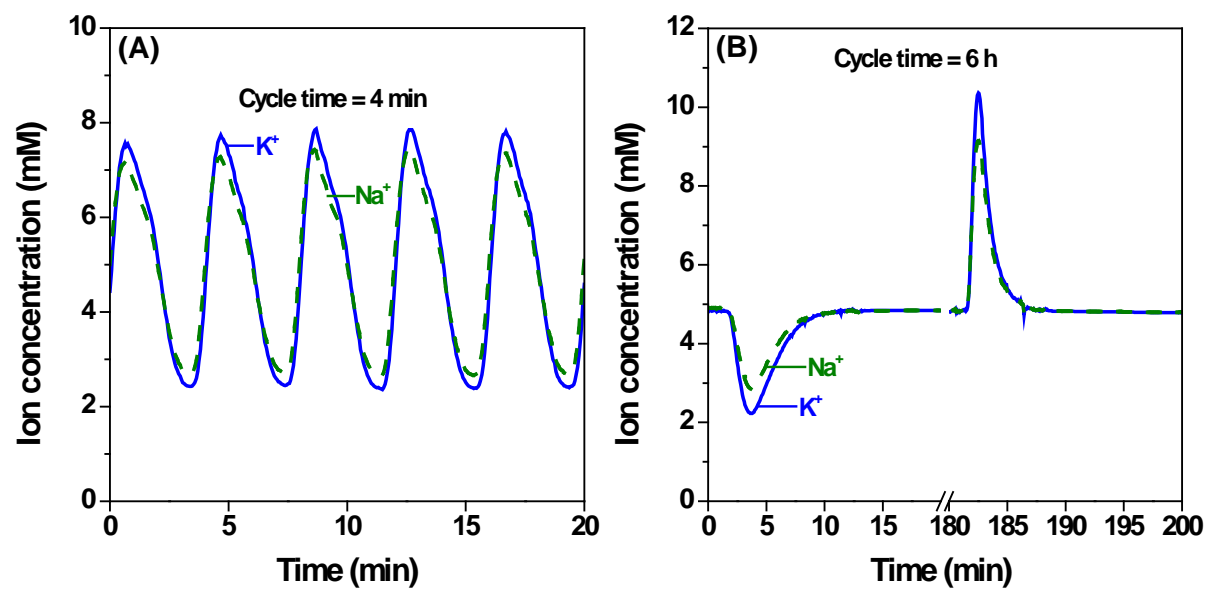

Fig. 5.2: Effluent ion concentration as function of time for experiments with a cycle time of $4 \mathrm{~min}(\mathrm{~A})$ and of $6 \mathrm{~h}(\mathrm{~B})$, during adsorption and desorption $\left(V_{\mathrm{ch}}=1.0 \mathrm{~V}, c_{\mathrm{in}, \mathrm{NaCl}}=5\right.$ $\mathrm{mM}$ and $\left.c_{\mathrm{in}, \mathrm{KCl}}=5 \mathrm{mM}\right)$.

can determine the effluent concentration of multiple ions very accurately, using a sampling interval time from 2 to $4 \mathrm{~s}$.

In Fig. 5.3A we present adsorption of $\mathrm{K}^{+}$and $\mathrm{Na}^{+}$ions as a function of CT and see that already at short CTs, the removal of $\mathrm{K}^{+}$ions from the feed water is higher than of $\mathrm{Na}^{+}$, while the concentration of both ions in the feed water is equal. Fig. 5.3B shows that our theory predicts the experimentally observed ion adsorption data very closely at short CTs, although data shows a faster increase in ion adsorption compared to theory (see Table 5.1 for parameter values used in our model).

The data shows that we reach the highest ratio of $\mathrm{K}^{+}$over $\mathrm{Na}^{+}$adsorbed with a CT of $7.5 \mathrm{~min}$. Whereas the data shows that a longer CT does not significantly change this ratio, our theory predicts that this ratio decreases to unity. This decrease can be explained by Eq. (2.1), which defines that, at equilibrium, the concentration of an ion in the micropores is a function of the concentration in bulk solution, the valence of an ion, and the Donnan potential. As the bulk concentrations of $\mathrm{K}^{+}$and $\mathrm{Na}^{+}$are equal, and the different ions experience the same Donnan potential, $\mathrm{K}^{+}$and $\mathrm{Na}^{+}$adsorption should be equal at equilibrium. Since data shows a different adsorption for $\mathrm{K}^{+}$and $\mathrm{Na}^{+}$, we performed additional experiments with another batch of electrodes at an CT of $120 \mathrm{~min}$. The data of these experiments is shown in Fig. 5.3A (open stars), and again, we find a ratio of $\mathrm{K}^{+}$over $\mathrm{Na}^{+}$higher than the ratio predicted by theory.

According to our theory, at equilibrium, selective ion adsorption can only be achieved if the ions have a different charge. To account for the discrepancy be- 

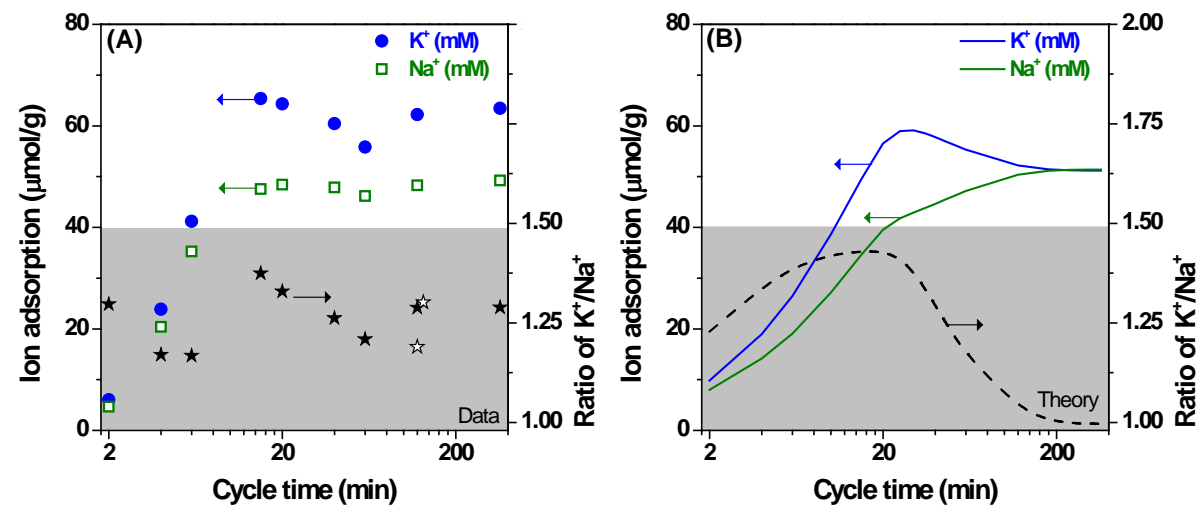

Fig. 5.3: (A) Experimental data for ion adsorption (blue circles for $\mathrm{K}^{+}$and green squares for $\mathrm{Na}^{+}$) and their ratio (black stars) plotted as function of cycle time. (B) Theoretical predictions of ion adsorption (blue line for $\mathrm{K}^{+}$and green line for $\mathrm{Na}^{+}$) and their ratio (black line), calculated based on the dynamic CDI model. In panel (A), open stars show ratio of $\mathrm{K}^{+}$over $\mathrm{Na}^{+}$for experiments with another batch of the same electrode material.

tween data and theory, one may include the effect of different chemical attractions of the carbon surface towards different ions.

To conclude, Fig. 5.3 shows that higher adsorption of one ion over another is possible, even though both ions have equal charge and very similar hydrated radius. This selective adsorption was achieved without modifying the electrode material or adding additional components to the system design, such as ionexchange membranes.

In Fig. 5.4 we show data and theory for charge and charge efficiency, which is the ratio of the total anion (or cation) adsorption over total charge, as function of the cycle time. Clearly, a longer cycle time gives rise to charge and charge efficiency. Whereas we find good agreement between theory and data of charge efficiency, the model underestimates charge.

\subsection{Conclusions}

We presented an on-line method to study dynamic ion adsorption from solutions of multiple salts in Capacitive Deionization (CDI), which has, to the best of our knowledge, not been introduced in CDI research before. With the presented method ion concentrations in the effluent of a CDI cell can be accurately measured with a sampling time of 2-4 s. We show that selective removal of $\mathrm{K}^{+}$over $\mathrm{Na}^{+}$is possible without modifying the electrode material or adding additional components to the system design. Finally, we validate our experimental data 

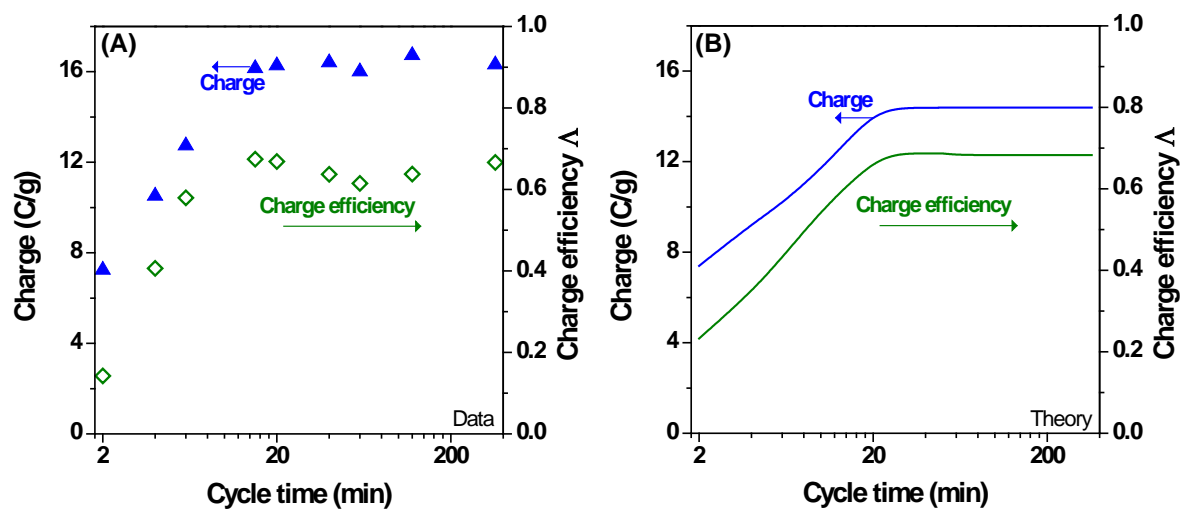

Fig. 5.4: Experimental data and theory of accumulated charge (data: blue triangles, theory: blue line), and charge efficiency, $\Lambda$, (data: green diamonds, theory: green line) as function of cycle time. (For interpretation of the references to color in this figure legend, the reader is referred to the web version of this article.)

with a dynamic CDI model and find a good correlation between data and theory at low cycle times. We also point out that theory and data show a significant difference for longer cycle times. 


\section{Effect of dissolved oxygen on carbon electrodes in CDI and MCDI}

This Chapter will be submitted:

J.E. Dykstra, M.K. Struijs, G.C. Franken, S. Porada, A. van der Wal, Effect of dissolved oxygen on porous carbon electrodes in (Membrane) Capacitive Deionization. 


\section{Abstract}

Capacitive Deionization (CDI) is an electrochemical desalination technology. In a CDI cell, water flows through a spacer channel which is placed between two electrodes, and by applying a voltage over the electrodes, ions are adsorbed. To enhance salt adsorption, membranes can be placed in front of the electrodes, a modification called Membrane Capacitive Deionization (MCDI). In a MCDI cell, ion exchange membranes are placed between electrodes and spacer channel. In this Chapter, CDI and MCDI cells treating water with and without dissolved oxygen are compared. Results show that the desalination performance of CDI (thus, without membranes) decreases over time, most rapidly when a cell treats feed water with dissolved oxygen. We describe experimental data with the amphoteric Donnan model and find that the model describes experimental data best with an increase in time of negative chemical surface charge in the anode and of positive charge in the cathode. Similar experiments for MCDI (thus, with membranes) show that, although dissolved oxygen affects the surface chemistry of the electrode material, the desalination performance does not decrease over time, which we explain by the fact that charge efficiency and desalination performance in MCDI are mainly determined by the selectivity of the membranes. 


\subsection{Introduction}

Capacitive Deionization (CDI) is an electrochemical desalination technology using electrodes that are alternately charged and discharged [2, 4-8, 168, 169]. A CDI cell consists of two porous carbon electrodes with a spacer channel in between. Feed water flows through the channel and by applying a charging voltage over the electrodes, anions are adsorbed into the electrically positively charged electrode, the anode, and cations into the negatively charged electrode, the cathode. Consequently, water is desalinated. After the electrodes are saturated with salt, they can be short-circuited for regeneration, which results in concentrated effluent stream.

In CDI with porous carbon electrodes, ions are adsorbed into electrical double layers (EDLs) that are formed in the micropores of the electrodes [29-32]. For each electron transported from one electrode to the other, either a counterion can be adsorbed into the EDL (desired), or a co-ion can be desorbed (undesired). To avoid the desorption of co-ions from the electrodes into the spacer channel, Membrane Capacitive Deionization (MCDI) was introduced [34-37]. In MCDI, a cation exchange membrane is placed in front of the cathode, and an anion exchange membrane in front of the anode, which block the transport of co-ions from electrodes into the spacer channel.

For CDI and MCDI, it is important to guarantee stable long-term desalination. Whereas MCDI has shown stable long-term performance [102], CDI shows a declining desalination performance over time, especially if dissolved oxygen is present in the feed water $[3,64,100,120,170,171]$. This decrease in desalination performance has been mainly attributed to faradaic reactions involving carbon oxidation [63-65].

In literature, several carbon oxidation reactions are described [60, 172-175]

$$
\begin{aligned}
\mathrm{C}+\mathrm{H}_{2} \mathrm{O} & \longrightarrow \mathrm{C}-\mathrm{OH}+\mathrm{H}^{+}+\mathrm{e}^{-} \\
\mathrm{C}+\mathrm{H}_{2} \mathrm{O} & \longrightarrow \mathrm{C}=\mathrm{O}+2 \mathrm{H}^{+}+2 \mathrm{e}^{-} \\
\mathrm{C}+2 \mathrm{H}_{2} \mathrm{O} & \longrightarrow \mathrm{O}=\mathrm{C}-\mathrm{OH}+3 \mathrm{H}^{+}+3 \mathrm{e}^{-} \\
\mathrm{C}+2 \mathrm{H}_{2} \mathrm{O} & \longrightarrow \mathrm{CO}_{2}+4 \mathrm{H}^{+}+4 \mathrm{e}^{-} .
\end{aligned}
$$

Eq. (6.3) is the production reaction of carboxylic (-COOH) groups, which can dissociate into carboxylate $\left(-\mathrm{COO}^{-}\right)$groups $[84,176,177]$. These carboxylate groups have a negative charge and result in the formation of negative chemical surface charge. The presence of these groups can affect the adsorption of ionic charge in EDLs, and can affect the salt adsorption performance. These oxidation reactions can only occur for a longer period of time if a reduction reaction also 
takes place, such as $[60,173]$

$$
\begin{aligned}
\mathrm{C}+\mathrm{H}_{2} \mathrm{O}+\mathrm{e}^{-} & \longrightarrow \mathrm{C}-\mathrm{H}+\mathrm{OH}^{-} \\
\mathrm{O}_{2}+2 \mathrm{H}_{2} \mathrm{O}+4 \mathrm{e}^{-} & \longrightarrow 4 \mathrm{OH}^{-} .
\end{aligned}
$$

In (M)CDI, other electrochemical reactions can occur as well, such as the production of $\mathrm{Cl}_{2}$ by [58-62]

$$
2 \mathrm{Cl}^{-} \longrightarrow \mathrm{Cl}_{2}(\mathrm{aq})+2 \mathrm{e}^{-}
$$

In present Chapter, we will not elaborate on these reactions.

In present Chapter, we study desalination in CDI and MCDI cells treating feed water with and without dissolved oxygen for at least 80 hours. Experimental results are compared with the amphoteric Donnan model. This model describes salt adsorption as function of cell voltage and considers chemical surface charge and other EDL parameters. We employ this model to study the change of chemical surface charge over time during desalination, and thus, to investigate whether we can describe the decreasing desalination performance during CDI experiments with a change in chemical surface charge, see Fig. 6.1.

As already introduced, desalination in MCDI shows, contrary to CDI, a stable long-term performance, also if dissolved oxygen is present in the feed water [102]. Although several researchers explain the stable performance by the fact that ion exchange membranes (IEMs) act as a barrier for oxygen to reach the electrode surface [120, 171], we hypothesize that oxygen passes the IEMs [178] and affects the surface chemistry of the carbon electrodes. However, contrary to CDI, the desalination performance does not decrease so rapidly in MCDI compared to CDI, as the IEMs act as a barrier for co-ions to leave the electrode region. In the present Chapter, we will test this hypothesis, and we will show that, also in MCDI, the surface chemistry of the electrodes is affected by oxygen.

\subsection{Theory}

The amphoteric Donnan model is used to describe desalination performance during long-term CDI experiments. This model calculates salt adsorption, charge density and charge efficiency, and relates these variables to cell voltage and electrical double layer (EDL) properties, which are micropore volume, Stern layer capacitance and chemical surface charge [33, 51].

Each long-term desalination experiment consists of a large number of desalination cycles, with each cycle consisting of an adsorption and a desorption step. Values for desalination performance as function of chemical surface charge are calculated at the end of each charging step, when equilibrium is reached. Thus, 


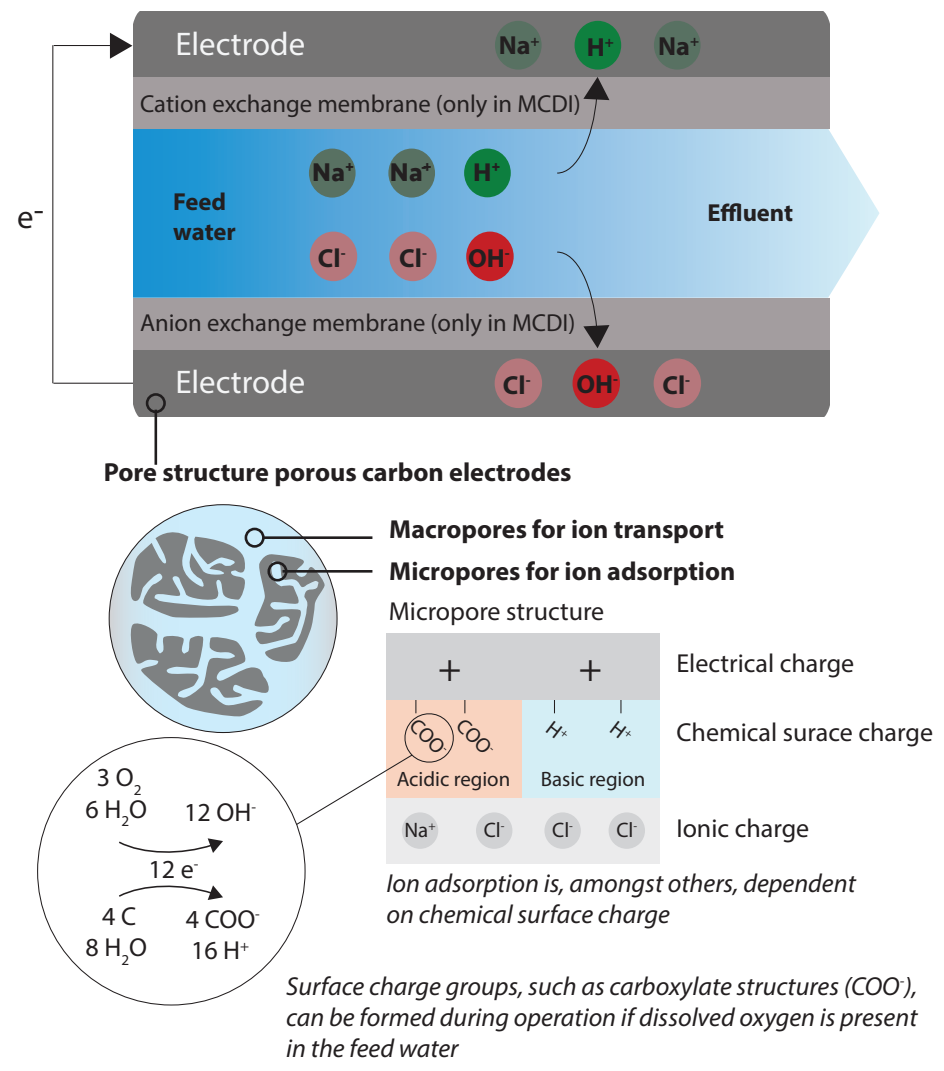

Fig. 6.1: Overview of (membrane) capacitive deionization. Feed water flows through a spacer channel placed between two porous carbon electrodes and, for MCDI, between two ion exchange membranes. Ions are transported from the spacer channel through ion exchange membranes and macropores of the electrodes, into the micropores where adsorption takes place. 
although the change of desalination performance and surface charge is modelled over time, values of these variables are only calculated in equilibrium, and an equilibrium model suffices.

\subsubsection{Amphoteric Donnan model}

We model a CDI cell with two electrodes that are electrically oppositely charged. The micropores in each electrode are divided into two regions: the acidic and basic region (Fig. 6.1) [51]. The acidic region has a negative chemical surface charge, which is due to the presence of acidic groups, such as carboxylate structures. The basic region has a positive chemical surface charge, which caused by basic groups. So, in total 4 micropore regions (two electrodes with each two regions) are modelled.

We assume that the electrolyte only contains a monovalent salt. In other words, we assume that the concentrations of carbonic acid species $\left(\mathrm{H}_{2} \mathrm{CO}_{3}, \mathrm{HCO}_{3}{ }^{-}\right.$and $\left.\mathrm{CO}_{3}{ }^{2-}\right)$, and of protons $\left(\mathrm{H}^{+}\right)$and hydroxyl ions $\left(\mathrm{OH}^{-}\right)$are very low and therefore their contribution to salt adsorption in the micropores is negligible ${ }^{1}$.

In each micropore region, three types of charge in the EDL are considered: electronic, ionic and chemical [33]. The electronic charge, $\sigma_{\text {elec }}$, is due to electrons stored in the carbon matrix, whereas the ionic charge, $\sigma_{\text {ionic }}$, is due to ions in the diffuse layer. The chemical charge, $\sigma_{\text {chem }}$, is due to acidic or basic groups at the carbon surface. For each micropore region, the sum of the different types of charge is equal to zero, thus

$$
\sigma_{\text {elec }}+\sigma_{\text {ionic }}+\sigma_{\text {chem }}=0 \text {. }
$$

The ionic charge is evaluated according to

$$
\sigma_{\text {ionic }}=-2 \cdot c_{\infty} \cdot \sinh \left(\Delta \phi_{\mathrm{D}}\right)
$$

where $\Delta \phi_{\mathrm{D}}$ is the Donnan potential between macro- and micropores, and where $c_{\infty}$ is the salt concentration in the bulk solution, which is, in equilibrium, equal to the concentration in the macropores of the electrodes. We relate the electronic charge to the Stern potential and Stern layer capacitance [33, 51]

$$
\sigma_{\text {elec }}=\Delta \phi_{\mathrm{S}} \cdot V_{\mathrm{T}} \cdot C_{\mathrm{S}} / F
$$

where $\Delta \phi_{\mathrm{S}}$ is the Stern potential and $C_{\mathrm{S}}$ the Stern layer capacitance. The thermal voltage, $V_{\mathrm{T}}$, is given by $V_{\mathrm{T}}=\frac{R \cdot T}{F}$, where $R$ is the gas constant $(8.314 \mathrm{~J} /(\mathrm{mol}$

\footnotetext{
${ }^{1}$ In Section 6.3 we describe that the concentration of carbonic acid species in solution is negligible compared to the concentration of $\mathrm{NaCl}$.
} 
$\mathrm{K})$ ), $T$ the temperature and $F$ Faraday's constant $(96,485 \mathrm{C} / \mathrm{mol})$. We calculate the total concentration of ions in the micropore region, $c_{\text {ions,mi }}$, using

$$
c_{\text {ions }, \mathrm{mi}}=2 \cdot c_{\infty} \cdot \cosh \left(\Delta \phi_{\mathrm{D}}\right)
$$

The EDL potential, $\Delta \phi_{\mathrm{EDL}}$, is the summation of the Donnan and Stern potential. This potential is considered equal for acidic and basic regions, and is evaluated for both anode and cathode using [51, 159]

$$
\Delta \phi_{\mathrm{EDL}}=\left(\Delta \phi_{\mathrm{D}}+\Delta \phi_{\mathrm{S}}\right)_{\mathrm{A}}=\left(\Delta \phi_{\mathrm{D}}+\Delta \phi_{\mathrm{S}}\right)_{\mathrm{B}}
$$

where subscript "A" refers to the acidic, and "B" to the basic region.

The cell voltage, $V_{\text {cell }}$, is related to $\Delta \phi_{\mathrm{EDL}}$ in the anode (indicated with subscript "an") and cathode ("cat")

$$
V_{\text {cell }}=V_{\mathrm{T}} \cdot\left(\Delta \phi_{\mathrm{EDL}, \text { an }}-\Delta \phi_{\mathrm{EDL}, \mathrm{cat}}\right)
$$

For each electrode the average electronic charge over the acidic and basic region, and the average ion concentration in the micropores are given by

$$
\begin{array}{rc}
\sigma_{\text {elec,avg }} & =\frac{1}{2}\left(\sigma_{\text {elec }, \mathrm{A}}+\sigma_{\text {elec }, \mathrm{B}}\right) \\
c_{\text {ions,mi,avg }} & =\frac{1}{2}\left(c_{\text {ions,mi }, \mathrm{A}}+c_{\text {ions, mi }, \mathrm{B}}\right) .
\end{array}
$$

Assuming that the acidic and basic micropore regions have equal volume in both electrodes, the average electronic charge in the anode and cathode sum up to zero,

$$
\sigma_{\text {elec,avg,an }}+\sigma_{\text {elec,avg,cat }}=0 \text {. }
$$

Theoretical values for salt adsorption, $\Gamma_{\text {salt }}$, charge density, $\Sigma_{\mathrm{F}}$, and charge efficiency, $\Lambda$, are given by

$$
\begin{aligned}
\Gamma_{\text {salt }} & =\frac{1}{4} \cdot M_{\mathrm{w}, \text { salt }} \cdot v_{\mathrm{mi}} . \\
& \left(\left.\left(c_{\text {ions,mi,avg }}^{\text {ch,end }}-c_{\text {ions,mi,avg }}^{\text {dch,end }}\right)\right|_{\text {ca }}+\left.\left(c_{\text {ions,mi,avg }}^{\text {ch,end }}-c_{\text {ions,mi,avg }}^{\text {dch,end }}\right)\right|_{\text {an }}\right) \\
\Sigma_{\mathrm{F}} & =\frac{1}{2} \cdot F \cdot v_{\mathrm{mi}}\left(\sigma_{\text {elec,avg }}^{\text {ch,end }}-\sigma_{\text {elec,avg }}^{\text {dch,end }}\right) \\
\Lambda & =\frac{F}{M_{\mathrm{w}, \text { salt }}} \cdot \frac{\Gamma_{\text {salt }}}{\Sigma_{\mathrm{F}}}
\end{aligned}
$$

where $c_{\text {ions,mi,avg }}^{\text {ch,end }}\left(c_{\text {ions,mi,avg }}^{\mathrm{dch}, \text { end }}\right)$ is $c_{\text {ions,mi,avg at the end of a charging (discharge) }}$ step, and where $M_{\mathrm{w} \text {,salt }}$ is the molar mass of the salt (for $\mathrm{NaCl} M_{\mathrm{w} \text {,salt }}=58.44$ $\mathrm{g} / \mathrm{mol}$ ). Please note that $\Sigma_{\mathrm{F}}$ can be calculated using values for $\sigma_{\text {elec,avg }}$ in anode or cathode. 


\subsubsection{Change of chemical surface charge}

To model the development of $\sigma_{\text {chem }}$, we assume that groups are formed in both regions, and that the formation rates are the same. Furthermore, we assume that the formation rate can be described using an exponential relationship. For each electrode, we use the relations

$$
\begin{aligned}
\sigma_{\text {chem,A }} & =\gamma_{\mathrm{A}}+\left(\sigma_{\text {chem,A }}^{\text {start }}-\gamma_{\mathrm{A}}\right) \cdot \exp (-k \cdot t) \\
\sigma_{\text {chem,B }}-\sigma_{\text {chem,B }}^{\text {start }} & =\sigma_{\text {chem,A }}-\sigma_{\text {chem,A }}^{\text {start }}
\end{aligned}
$$

where $\gamma$ describes the maximum value of $\sigma_{\text {chem }}$, or, in other words, the asymptote of the function, and $k$ describes the rate of formation. Both $\gamma$ and $k$ are fitting parameters. Parameter $\sigma_{\text {chem }}^{\text {start }}$ is the value of $\sigma_{\text {chem }}$ of pristine electrodes, which are electrodes that have not been charged before. Variable $t$ is the time to reach the end of a desalination cycle. To calculate the evolution of variables $\sigma_{\text {chem,A }}$ and $\sigma_{\text {chem,B }}$ during an experiment, we compute values for these variables at the end of each desalination cycle. Thus, $t$ runs from $t_{\text {cycle }}$ to $t_{\text {end }}$ in discrete steps of $t_{\text {cycle }}$, where $t_{\text {cycle }}$ is the duration of a desalination cycle, and $t_{\text {end }}$ the total time of the experiment.

\subsection{Materials and methods}

\subsubsection{CDI experiments}

CDI experiments were conducted using a stack containing 4 CDI cells, which was placed in a polymethylmethacrylate (PMMA) housing. Each CDI cell consisted of two home-made porous carbon electrodes (geometric surface area $33.75 \mathrm{~cm}^{2}$ ), and a spacer material (AP20, Glass Fiber Filter, Millipore, MA, thickness 300 $\mu \mathrm{m})$ positioned between the electrodes. The electrodes were fabricated according to the procedure described in Chapter 2. The cells were connected with the electrical circuit by placing the electrodes between graphite current collectors.

We pumped a $\mathrm{NaCl}$ solution (temperature $295 \mathrm{~K}$ ) from a recirculation vessel of $10 \mathrm{~L}$ through the CDI cell, and back to the recirculation vessel with a flow rate of $7.5 \mathrm{~mL} / \mathrm{min} /$ cell. At the outflow of the cell, we placed a conductivity and $\mathrm{pH}$ sensor, which measured at a sampling time of $1 \mathrm{~s}$. The salt solution was prepared with a concentration of $20 \mathrm{mM}$, and the $\mathrm{pH}$ was controlled, during CDI experiments, at a value between 6.8 and $7.2 .^{2}$

We conducted experiments under deaerated and aerated conditions. For the deaerated experiments, we purged the inflow solution with nitrogen to strip dissolved oxygen, while for the aerated experiments, we purged the inflow solution

\footnotetext{
${ }^{2}$ For the MCDI experiments, we did not control the $\mathrm{pH}$ of the inflow solution.
} 
with air. We note that, although carbonic acid species are present in the inflow of aerated experiments, the total concentration is low $(0.06 \mathrm{mM}$ at $\mathrm{pH} 7)$ compared to the $\mathrm{NaCl}$ concentration, and therefore, these species are not considered for the calculation of salt adsorption.

The electrical circuit of the CDI stack was connected with a potentiostat (IviumStat, Ivium Technologies, The Netherlands) to control cell voltage and measure current. We alternately charged and discharged the cell. Charging was done at a constant voltage of $1.2 \mathrm{~V}$ for $400 \mathrm{~s}$; discharge was done at $0 \mathrm{~V}$ for $250 \mathrm{~s}$.

\subsubsection{MCDI and MCDI-I experiments}

The MCDI experiments were conducted following the procedure described in Section 6.3.1, with a stack containing 4 MCDI cells. Each MCDI cell was built just as a CDI cell, but a cation exchange membrane (Neosepta CMX, thickness $190 \mu \mathrm{m})$ was placed between spacer and cathode, and an anion exchange membrane (Neosepta AMX, thickness $130 \mu \mathrm{m}$ ) between spacer and anode. The MCDI experiments were performed under aerated conditions.

To determine the effect of dissolved oxygen on electrodes placed in an MCDI cell, we temporarily paused the MCDI experiment and removed the membranes from the stack. Then, we conducted intermediate CDI experiments, referred to as MCDI-I, to test the desalination performance of the electrodes. These MCDI-I experiments were performed under deaerated conditions to avoid the presence of dissolved oxygen and thus to minimize the occurrence of faradaic reactions. The cell was alternately charged and discharged as described in Section 6.3.1 for 3 cycles (total duration $1950 \mathrm{~s}$ ). Finally, membranes were re-inserted into the stack and the MCDI experiment was continued. An overview of the experimental design of these MCDI with MCDI-I experiments conducted intermediately is shown in Table 6.1.

\subsubsection{Desalination performance}

The desalination performance is expressed in terms of salt adsorption, charge density and charge efficiency. The salt adsorption of each (M)CDI cycle was calculated by integrating the difference between effluent and inflow salt concentration over time during an adsorption step, and multiplying the resulting value with the flow rate through the (M)CDI stack and the molar mass of the salt.

In this Chapter, we distinguish two types of charge: capacitive and faradaic. The capacitive charge, $\Sigma_{\mathrm{F}, \mathrm{c}}$, is stored in EDLs, and the faradaic charge, $\Sigma_{\mathrm{F}, \mathrm{f}}$, is used to drive faradaic reactions, such as Eqs. (6.1)-(6.6). During the charging step (charging voltage $1.2 \mathrm{~V}$ ) these reactions could occur, which means that the charge at the end of a charging step is the sum of capacitive and faradaic charge, 
Table 6.1: Experimental design of MCDI-Aerated experiments with intermediate CDI experiments (referred to as MCDI-I). The MCDI-Aerated experiments were performed with an inflow solution containing dissolved oxygen (aerated), whereas MCDI-I experiments were performed with an inflow solution stripped from dissolved oxygen (deaerated). The aim of the MCDI-I experiments of was to determine the desalination performance of the electrodes without the inclusion of IEMs in the cell, and thereby study the effect of dissolved oxygen on electrodes in MCDI.

\begin{tabular}{|l|cccc|}
\hline & Duration & Cell design & Inflow solution & Aim \\
\cline { 2 - 5 } MCDI - I & $1950 \mathrm{~s}$ & CDI & Deaerated & Characterization \\
MCDI - Aerated & $2 \mathrm{~d}$ & MCDI & Aerated & Operation \\
MCDI - I & $1950 \mathrm{~s}$ & CDI & Deaerated & Characterization \\
MCDI - Aerated & $2 \mathrm{~d}$ & MCDI & Aerated & Operation \\
MCDI - I & $1950 \mathrm{~s}$ & CDI & Deaerated & Characterization \\
\hline
\end{tabular}

which we call total charge, $\Sigma_{\mathrm{F}, \mathrm{t}}$. During the discharge, we assume that these reactions did not occur, which means that we only measured capacitive charge. The total, capacitive, and faradaic charge can be calculated using

$$
\begin{aligned}
& \Sigma_{\mathrm{F}, \mathrm{t}}=\Sigma_{\mathrm{F}, \mathrm{c}}+\Sigma_{\mathrm{F}, \mathrm{f}} \\
& \Sigma_{\mathrm{F}, \mathrm{t}}=\frac{1}{M_{\text {elec }}} \int_{0}^{t_{\mathrm{ch}}} I(t) \mathrm{d} t \\
& \Sigma_{\mathrm{F}, \mathrm{c}}=\frac{1}{M_{\text {elec }}} \int_{0}^{t_{\mathrm{dch}}} I(t) \mathrm{d} t
\end{aligned}
$$

where $M_{\text {elec }}$ is the total mass of all electrodes, $t_{\mathrm{ch}}$ is the duration of the charging step, and $t_{\mathrm{dch}}$ of the discharge step. To calculate charge efficiency, we use Eq. (6.20), where we insert $\Sigma_{\mathrm{F}, \mathrm{c}}$ for $\Sigma_{\mathrm{F}}$.

\subsection{Results and discussion}

\subsubsection{CDI}

The desalination performance expressed in terms of salt adsorption, charge efficiency and capacitive charge of a desalination cycle is shown in Fig. 6.2. Experiments were conducted with aerated and deaerated feed water. Clearly, a decrease of desalination performance is observed over time, which is for the aerated case stronger than for the deaerated case, and is in agreement with other studies [3, 64, 100, 120, 170, 171]. After 45 hours of desalination, for the aerated case, salt adsorption becomes negative, which means that salt is adsorbed during discharge (charging voltage $0 \mathrm{~V}$ ), and desorbed during charging (discharge 

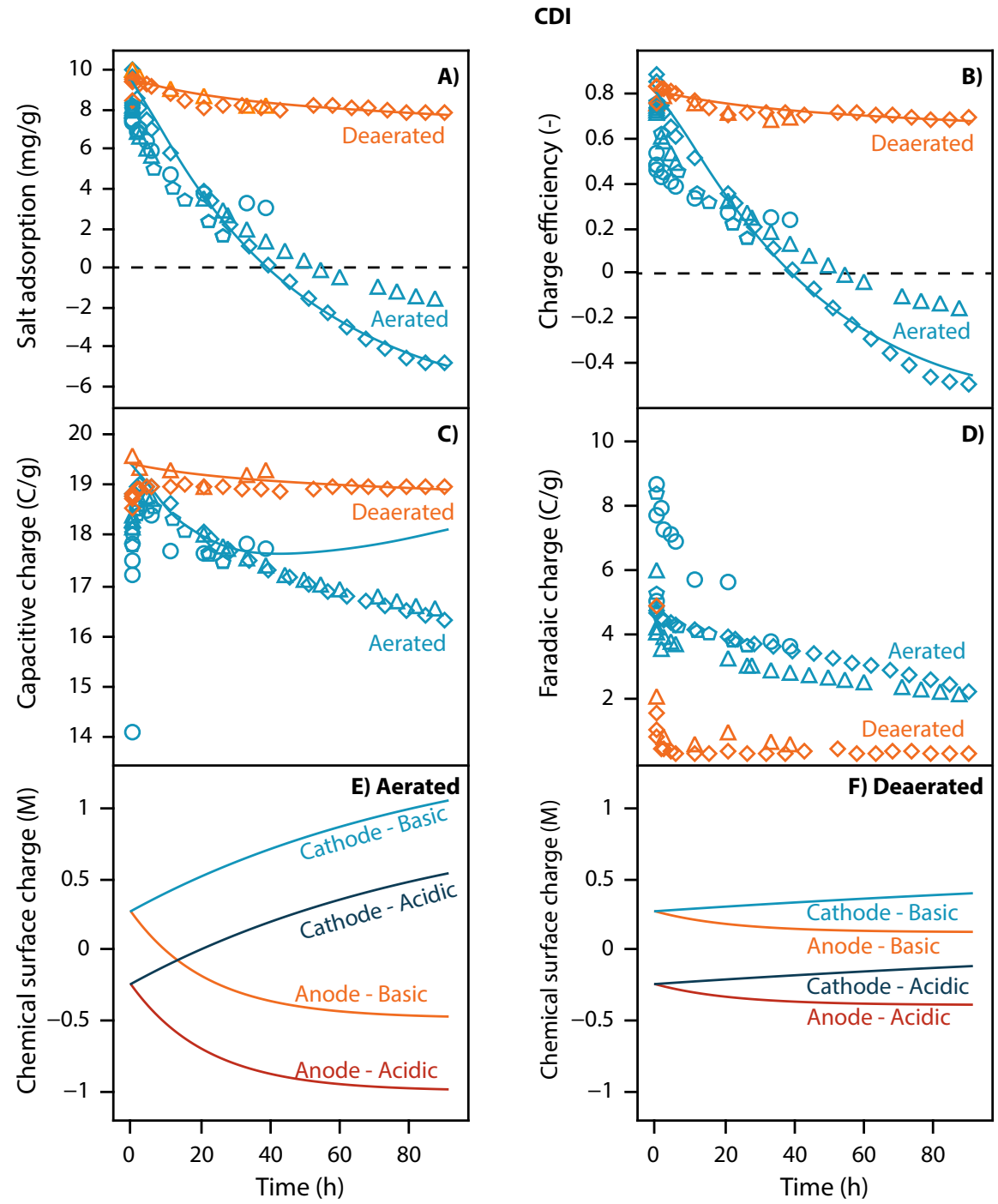

Fig. 6.2: Experimental data and theory of a) salt adsorption, b) charge efficiency, c) capacitive charge, and d) faradaic charge per cycle as function of desalination time for CDI. Experiments were conducted with aerated and deaerated feed water. Various series of experiments were conducted, each indicated with a different symbol. Chemical surface charge calculated by the amphoteric Donnan model to the describe CDI experiments conducted with aerated $(\mathrm{E})$ and deaerated feed water $(\mathrm{F})$. 
Table 6.1: Parameters used for amphoteric Donnan model

\begin{tabular}{|c|c|c|c|}
\hline \multicolumn{4}{|c|}{ General electrode parameters } \\
\hline$v_{\mathrm{mi}}$ & Micropore volume & 0.45 & $\mathrm{~mL} / \mathrm{g}$ \\
\hline$C_{\mathrm{S}}$ & Stern capacitance & 160 & $\mathrm{~F} / \mathrm{mL}$ \\
\hline$\sigma_{\text {chem,A }}^{\text {start }}$ & $\begin{array}{l}\text { Chemical surface charge acidic region } \\
\text { at start (both anode and cathode) }\end{array}$ & -239 & $\mathrm{mM}$ \\
\hline$\sigma_{\text {chem,B }}^{\text {start }}$ & $\begin{array}{l}\text { Chemical surface charge basic region } \\
\text { at start (both anode and cathode) }\end{array}$ & 274 & $\mathrm{mM}$ \\
\hline$\alpha_{\mathrm{mi}, \mathrm{A}}=\alpha_{\mathrm{mi}, \mathrm{B}}$ & $\begin{array}{l}\text { Volume ratio acidic and basic } \\
\text { regions micropores }\end{array}$ & $1: 1$ & \\
\hline$T$ & Temperature & 298 & K \\
\hline \multicolumn{4}{|c|}{ Formation chemical surface charge at electrode surface } \\
\hline & Aerated case & \multicolumn{2}{|c|}{ Deaerated case } \\
\hline \multicolumn{4}{|l|}{ Anode } \\
\hline$\gamma_{\mathrm{A}}$ & $-992 \mathrm{mM}$ & -387 & $\mathrm{mM}$ \\
\hline$k$ & $0.046 \mathrm{~s}^{-1}$ & 0.046 & $\mathrm{~s}^{-1}$ \\
\hline \multicolumn{4}{|l|}{ Cathode } \\
\hline$\gamma_{\mathrm{A}}$ & $1000 \mathrm{mM}$ & 156 & $\mathrm{mM}$ \\
\hline$k$ & 0.011 & 0.004 & $\mathrm{~s}^{-1}$ \\
\hline
\end{tabular}

voltage $1.2 \mathrm{~V}$ ). Theory is in good agreement with data for salt adsorption and charge efficiency, but not for capacitive charge after $40 \mathrm{~h}$ of desalination.

We described decreasing desalination performance by a change of chemical surface charge in acidic and basic regions, both for the aerated and deaerated case. Therefore, desalination data was fitted with theory to find values for $\gamma_{\mathrm{A}}$ and $k$ for each electrode, which are reported in Table 6.1. As Fig. 6.2 shows, in both cases, chemical surface charge decreases in the anode, and increases in the cathode, but the changes are much stronger for the aerated case than for the deaerated case. Note that the difference in surface charge between acidic and basic groups is constant, which we assumed and defined by Eq. (6.22).

The calculated decrease of chemical surface charge in the anode is most likely caused by carbon oxidation, see Eq. (6.3), which results in the formation of carboxylate surface groups with negative charge. However, the increase of surface charge at the cathode is difficult to explain; we have not yet identified the groups that can be formed at the cathode and have a positive chemical surface charge.

The data for effluent $\mathrm{pH}$ over time of different desalination cycles show that, at the start of an experiment (Fig. 6.3A, $0.5 \mathrm{~h}$.), there is a strong increase of effluent pH during charging. Later, after 96 h., pH fluctuations are very limited. 


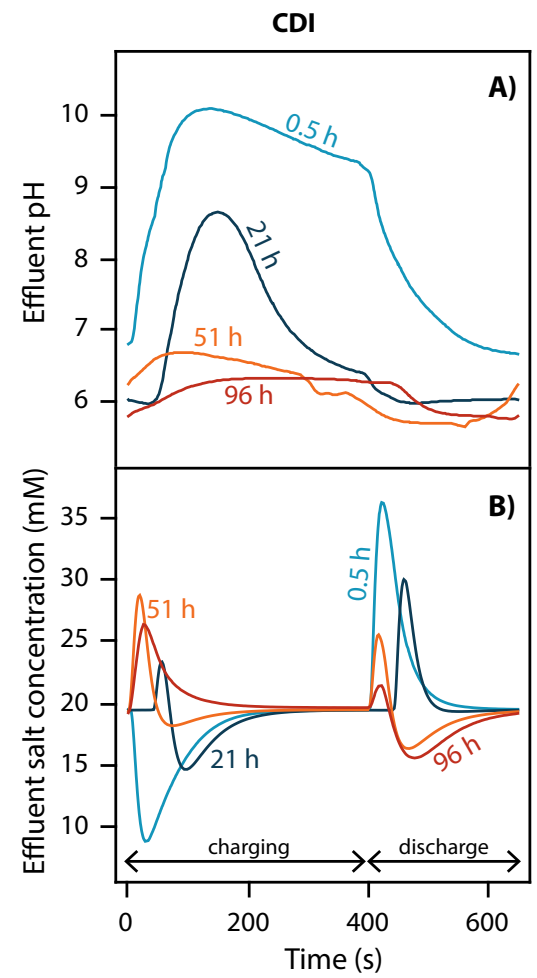

Fig. 6.3: Effluent $\mathrm{pH}$ and effluent salt concentration as function of time of various desalination cycles in CDI. ${ }^{3}$

These fluctuations can be caused by faradaic reactions, see Eqs. (6.1)-(6.7), if the combination of oxidation and reduction reactions results (temporarily) in the nett formation of $\mathrm{H}^{+}$or $\mathrm{OH}^{-}$. Furthermore, these fluctuations can be due to different mobilities of ions in solution $\left(\mathrm{Na}^{+}, \mathrm{Cl}^{-}, \mathrm{H}^{+}\right.$and $\left.\mathrm{OH}^{-}\right)$, which results in different ion adsorption rates. For MCDI, however, this fact explains $\mathrm{pH}$ fluctuations only to a small extent, as well will discuss in Chapter 7.

We also studied dynamics of salt adsorption, see Fig. 6.3B. The first cycles of a CDI aerated experiment show salt adsorption during charging, and salt desorption during discharge. However, in less than $21 \mathrm{~h}$ we observe inversion behavior: before salt adsorption, there is a desorption peak [64, 100]. Later, after $96 \mathrm{~h}$, during charging, there is only desorption of salt, and salt is adsorbed during the discharge step.

${ }^{3}$ The results show the dynamics of experiments for which equilibrium data is presented in Fig. 6.2 (dataset indicated with ' $\diamond$ '). 


\subsubsection{MCDI}

After studying the effect of aerated feed water on the desalination performance in CDI, we also studied the effect in MCDI. The results are shown in Fig. 6.4. Column I shows that salt adsorption (A) and charge efficiency (C) of MCDI aerated experiments decrease only slightly over time compared to CDI aereated.

We hypothesize that, although the desalination performance does not decrease in MCDI, the surface chemistry of the electrodes is still affected by dissolved oxygen. To test this hypothesis, MCDI-I experiments were conducted after two days of MCDI desalination. To that end, the MCDI experiment was paused, membranes were removed from the stack and we tested the desalination performance of the electrodes as described in Section 6.3.2. As Fig. 6.4 shows, we observe a decrease in desalination performance during the experiment compared to the CDI deaerated case. This finding indicates that, although membranes were present, the electrodes were affected by dissolved oxygen, and that dissolved oxygen passes through the IEMs, which is also supported by Refs. [178-180]. Because of the presence of IEMs, however, the desalination performance is not affected, as coions desorbed from the EDLs are trapped in the electrodes, they cannot flow from the electrodes into the spacer channel and the charge efficiency is not considerably affected by the change in surface chemistry at the electrodes. The most important parameter describing the charge efficiency in MCDI is the selectivity of the membranes, which indicates how many co-ions can pass the membranes and leave the electrode region. Thus, the charge efficiency of MCDI experiments is mainly determined by the selectivity of the membranes, not by the surface chemistry of the electrodes.

\subsection{Conclusions}

We showed that the desalination performance, expressed in terms of salt adsorption, charge density and charge efficiency, decreases over time in CDI. This decrease is much stronger in cells treating water containing dissolved oxygen, than in cells treating water without dissolved oxygen. Data of desalination experiments were described by the amphoteric Donnan model, and this model explains that the decrease in desalination performance is due to I) an increase of negative chemical surface charge in the anode, and II) an increase of positive chemical surface charge in the cathode. The first finding can be explained by the formation of $\mathrm{COO}^{-}$-groups in the anode, we do not know which groups are responsible for the second finding.

\footnotetext{
${ }^{4} \mathrm{CDI}$ aerated dataset indicated with ' $\diamond$ ' in Fig. 6.2.

${ }^{5} \mathrm{CDI}$ deaerated dataset indicated with ' $\diamond$ ' in Fig. 6.2.
} 

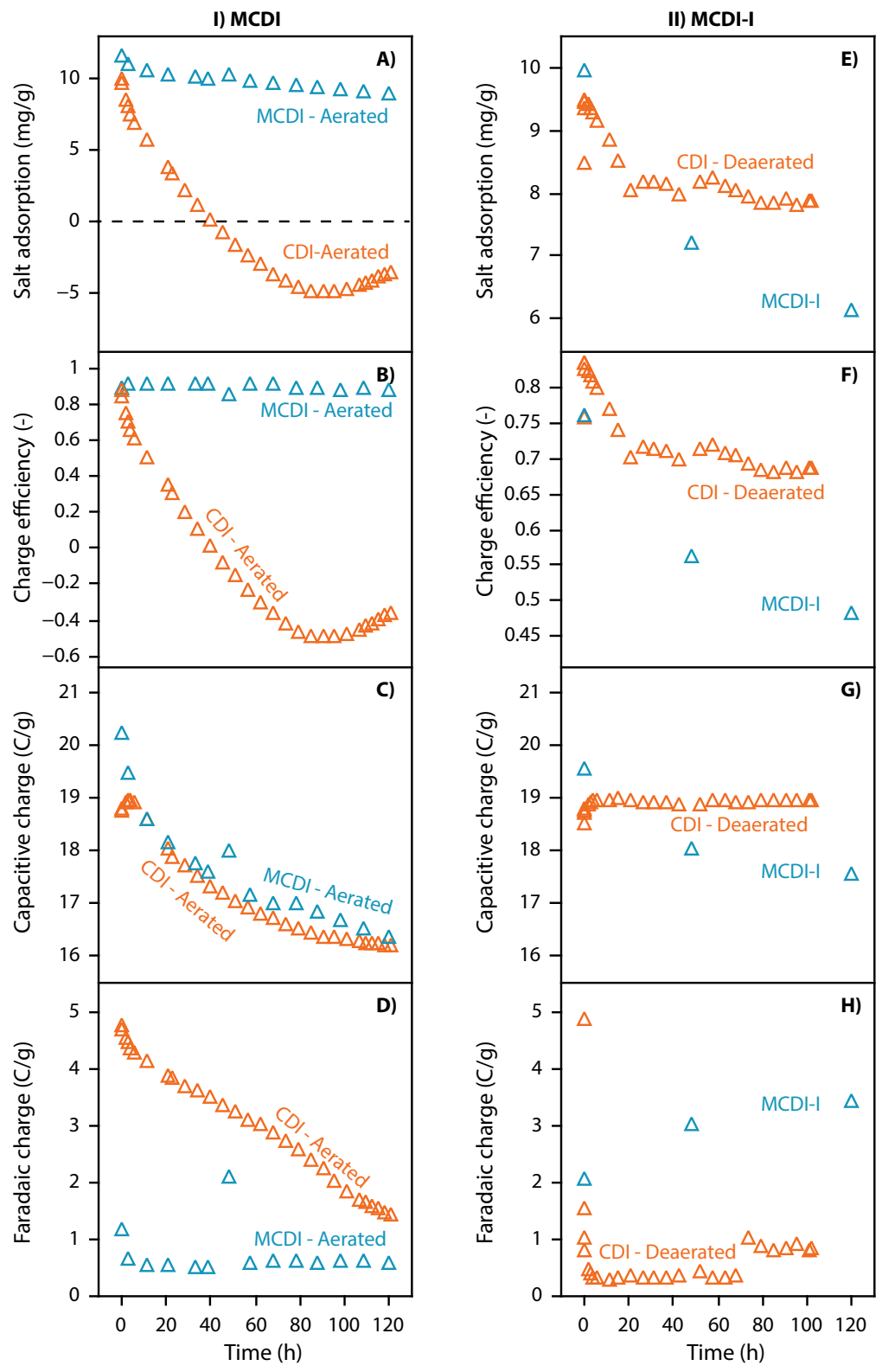

Fig. 6.4: MCDI desalination performance expressed in terms of salt adsorption, charge efficiency, capacitive charge, and faradaic charge as function of desalination time. Column I) shows the performance of aerated MCDI experiments, compared with aerated CDI experiments. ${ }^{4}$ Column II) shows the performance of MCDI-I experiments, which are intermediately conducted experiments without membranes under deaerated conditions to evaluate the performance of the electrodes. The performance of MCDI-I experiments is compared with deaerated CDI experiments. ${ }^{5}$ 
We also studied the effect of dissolved oxygen in MCDI and made the observation that, although the desalination performance does not decrease, the surface chemistry of the electrodes is affected by dissolved oxygen, which should in principle result in a lower charge desalination performance. However, as the ion exchange membranes acts as a barrier for ions to leave the electrode region, the selectivity of the membranes determines the charge efficiency of the process, rather than the surface chemistry of the electrodes. 


\section{Theory of $\mathrm{pH}$ changes in water desalination by}

MCDI

This Chapter has been published as:

J.E. Dykstra, K.J. Keesman, P.M. Biesheuvel, A. van der Wal, Theory of pH changes in water desalination by capacitive deionization, Water Research 119 (2017) 178-186. 


\section{Abstract}

In electrochemical water desalination, a large difference in $\mathrm{pH}$ can develop between feed and effluent water. These $\mathrm{pH}$ changes can affect the long-term stability of membranes and electrodes. Often faradaic reactions are implicated to explain these $\mathrm{pH}$ changes. However, quantitative theory has not been developed yet to underpin this idea. We develop a theory for electrochemical water desalination which includes not only faradaic reactions but also the fact that all ions in the water, including $\mathrm{H}^{+}$and $\mathrm{OH}^{-}$, have different mobilities (diffusion coefficients). We quantify the latter effect by microscopic physics-based modeling of $\mathrm{pH}$ changes in Membrane Capacitive Deionization (MCDI), a water desalination technology employing porous electrodes and ion-exchange membranes. We derive a dynamic model and include the following phenomena: I) different mobilities of various ions, combined with acid-base equilibrium reactions; II) chemical surface charge groups in the micropores of the porous carbon electrodes, where electrical double layers are formed; and III) faradaic reactions in the micropores. The theory predicts small $\mathrm{pH}$ changes during desalination cycles in MCDI if we only consider phenomena I) and II), but predicts that these $\mathrm{pH}$ changes can be much stronger if we consider phenomenon III) as well, which is in line with earlier statements in the literature on the relevance of faradaic reactions to explain $\mathrm{pH}$ fluctuations. 


\subsection{Introduction}

During water desalination by methods such as electrodialysis and capacitive deionization, often large changes in $\mathrm{pH}$ develop between feed and effluent water. These $\mathrm{pH}$ changes can result in precipitation and scaling of membranes and electrodes, and can affect long-term stability of membrane and electrode materials $[64,66-68]$. Also, these $\mathrm{pH}$ changes can lead to a product that has an undesired $\mathrm{pH}$.

In literature, different explanations can be found for these $\mathrm{pH}$ changes. Some studies describe how faradaic reactions result in production or consumption of protons or hydroxyl ions [8, 99-101], such as the reduction of water, the oxidation of carbon, the reduction of oxygen, or the oxidation of chloride ions. In our view another effect should be considered as well, which is the fact that all ions in the water have different mobilities (diffusion coefficients), which results in different ion adsorption rates, and thus in $\mathrm{pH}$ changes. We like to quantify these effects of different ion mobilities and faradaic reactions by microscopic physics-based modeling of a relevant electrochemical water desalination method.

To elucidate this effect, we choose to study $\mathrm{pH}$ changes in Membrane Capacitive Deionization (MCDI), a water desalination technology employing porous electrodes and ion-exchange membranes (IEMs) [2, 4-7, 72, 89, 168, 169]. As electrode material, we will focus on carbon electrodes. In MCDI, during the charging step, or adsorption step, a voltage is applied between electrodes, resulting in cation adsorption into the cathode, and anion adsorption into the anode. Consequently, feed water flowing through the cell is desalinated. Ions are adsorbed in the micropores of the electrodes, where electrical double layers (EDLs) are formed [29-31]. After the electrodes are saturated with salt, they are discharged and ions desorb. In MCDI, strong pH changes are often observed and feed water and effluent may have large differences in $\mathrm{pH}$, changing over time $[60,99,100]$. The IEMs are placed between the spacer channel and electrodes, see Fig. 7.1, and enhance salt adsorption [34, 35, 181].

To study $\mathrm{pH}$ changes in MCDI, we extend existing models, as described in Chapters 2, 3 and 5, and include besides $\mathrm{Na}^{+}$and $\mathrm{Cl}^{-}$ions (or any other pair of salt ions) also protons and hydroxyl ions. We model the transport of these ions across the spacer channel and membranes, into the electrodes. Furthermore, we consider the self-ionization reaction of water, $\mathrm{H}_{2} \mathrm{O} \rightleftarrows \mathrm{H}^{+}+\mathrm{OH}^{-}$. In the electrodes, we distinguish two types of pores: macropores and micropores. Macropores are large pores serving as transport pathways across the electrode [56, 142, 182]. Micropores are small pores where ions adsorb and faradaic reactions occur. To model ion adsorption, we consider the effect of chemical surface charge $[33,51]$. Chemical surface charge is present in the form of acidic groups, 


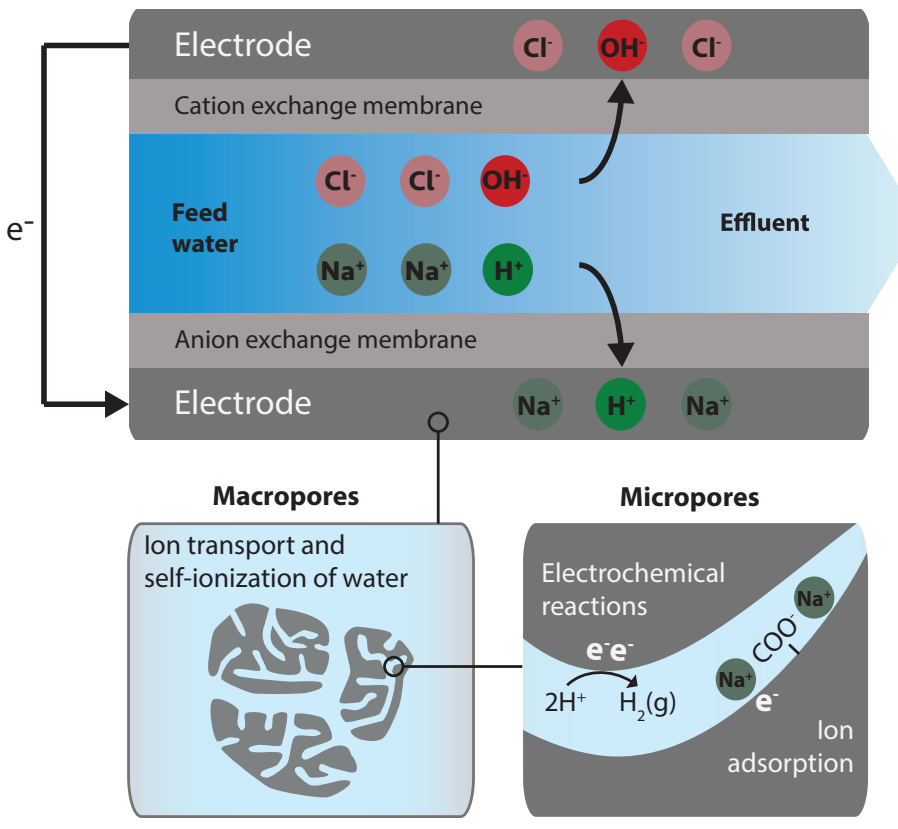

Fig. 7.1: Overview of membrane capacitive deionization. During charging, ions, including protons and hydroxyl ions, are transported through the membranes and macropores, and are adsorbed in the micropores. Each ion has a different transport rate, resulting in large differences in $\mathrm{pH}$ between feed water and effluent of the cell. Carboxylic and protonated structures are present in the micropores with a chemical surface charge. Faradaic reactions occur in the micropores at the carbon-solution interface. 
because of carboxylic structures [9, 90], or basic groups, because of protonated structures bound to carbon particles [183, 184].

We show that theory including the effect of different ion mobilities and the effect of acidic and basic groups in micropores, predicts differences in $\mathrm{pH}$ between feed water and effluent, which change over time. Furthermore, we show that these differences in $\mathrm{pH}$ can be much stronger if we also consider faradaic reactions.

\subsection{Theory}

To model $\mathrm{pH}$ in the MCDI cell as function of position (in membrane, spacer channel, and electrode) and time, we present a mathematical framework to calculate transport, adsorption and faradaic reactions of ions. This framework consists of three elements:

I) Transport of ions from the spacer channel, through the membranes, into the porous carbon electrodes, which is modelled based on the Nernst-Planck equation. In the present Chapter, we include acid-base equilibrium reactions between ions, such as the self-ionization reaction of water to protons and hydroxyl ions (and back). Acid-base equilibria can be included in several ways. In previous work we argued that for various reasons it is advantageous to assume that all acidbase reactions are locally at equilibrium $[143,145]$. In this approach, kinetic rate constants are not necessary and we only need to know pK-values of the respective chemical equilibria [185-187]. In the present Chapter, we follow this approach.

II) Adsorption of ions in the micropores of porous carbon electrodes, where EDLs are formed, which is modelled using the amphoteric Donnan model [33, 188]. This model relates salt adsorption, charge density and potential to one another, and includes the effect of chemical surface charge in the electrodes.

III) Faradaic reactions of ions in the micropores are modelled using the FrumkinButler-Volmer (FBV) equation $[40,69,70]$. This model relates kinetics of faradaic reactions self-consistently to local ion concentrations and electronic charge, without requiring knowledge of reference states.

Next, we present a theory including these three elements, i.e., the transport model including acid-base equilibria, the amphoteric Donnan model and the FBV equation. We consider the asymmetry between anode and cathode that can develop because of unequal ion diffusion coefficients, and because of different faradaic reaction kinetics. Therefore, we solve the equations presented in this section for each electrode (and membrane) separately. 


\subsubsection{Spacer channel}

In the spacer channel, transport of ions is evaluated in two directions: first, in the flow direction ( $y$-direction), thus, from entrance to exit of the cell, and second, in the direction perpendicular to flow ( $x$-direction), thus, across the width of the spacer channel. To describe changes in the $y$-direction mathematically, we divide the spacer channel into $M$ sub-cells, and we model that the electrolyte flows downstream from sub-cell $m$ into sub-cell $m+1$ [35, 189]. The concentration and $\mathrm{pH}$ of the electrolyte that leaves a certain sub-cell $m$ is equal to the concentration and $\mathrm{pH}$ in that particular sub-cell, and equal to the inflow concentration of subcell $m+1, c_{\text {inflow, } i}$. The effluent concentration of the cell is equal to the effluent concentration of sub-cell $M$. Note that the sub-cells are not mixed in the $x$ direction, but all concentrations and other properties remain $x$-dependent; thus, parallel streamlines are assumed. For each of these mathematical sub-cells, we dynamically evaluate all equations presented in this section. In this Chapter, we assume that the spacer channel is $100 \%$ open.

To describe concentration profiles in the direction perpendicular to the flow direction, thus in $x$-direction, we evaluate the molar flux of ions across the spacer channel, as given by the Nernst-Planck equation

$$
J_{\mathrm{sp}, i}=-D_{\mathrm{eff}, i}\left(\frac{\partial c_{\mathrm{sp}, i}}{\partial x}+z_{i} \cdot c_{\mathrm{sp}, i} \cdot \frac{\partial \phi_{\mathrm{sp}}}{\partial x}\right)
$$

where $c_{\mathrm{sp}, i}$ is the concentration of ion $i$ in the spacer channel, and $z_{i}$ is the valence of an ion. Parameter $D_{\text {eff }, i}$ is the effective diffusion coefficient, which in an open spacer channel is equal to $D_{i}$, which is the diffusion coefficient in free solution. We neglect tortuosity effects in spacer and electrodes. Variable $\phi_{\mathrm{sp}}$ is the dimensionless potential in the spacer channel, and $x$ runs from the spacer channel-membrane boundary on the anode-side, $\mathrm{CH} / \mathrm{M}_{\mathrm{an}}$, to the spacer channel-membrane boundary on the cathode-side, $\mathrm{S} / \mathrm{M}_{\text {cat }}$.

In the spacer channel, we assume a plug-flow profile for the fluid velocity of the water in $y$-direction, i.e., the flow velocity is invariant across the width of the channel, and for $\mathrm{Na}^{+}$and $\mathrm{Cl}^{-}$we set up a mass balance for each sub-cell (numbered 1...M), which is for an open channel given by

$$
\frac{\partial c_{\mathrm{sp}, i}}{\partial t}=-\frac{\partial J_{\mathrm{sp}, i}}{\partial x}+\frac{1}{\tau_{\mathrm{sc}}} \cdot\left(c_{\mathrm{inflow}, i}-c_{\mathrm{sp}, i}\right)
$$

where $c_{\text {inflow, } i}$ the inflow concentration of ion $i$ in the sub-cell (equal to the concentration in the previous sub-cell, at that particular $x$-position), and $\tau_{s c}$ is the retention time in a sub-cell. This retention time is given by $\frac{A_{\text {cell }} \cdot L_{\mathrm{sp}}}{\Phi_{\mathrm{v}} \cdot M}$, where $A_{\text {cell }}$ is the electrode geometric surface area, $L_{\mathrm{sp}}$ the thickness of the spacer channel, $\Phi_{\mathrm{v}}$ the flowrate through the cell, and $M$ the number of mathematical 
sub-cells. Note that concentrations and fluxes, $c_{\mathrm{sp}, i}$ and $J_{\mathrm{sp}, i}$, in Eq. (7.2), are all $x$-dependent. Boundary conditions for Eq. (7.2) will be discussed in Section 7.2.2 (Eqs. (7.11) and (7.12)).

At each position in the spacer channel, we assume that the electroneutrality condition

$$
\rho_{\mathrm{sp}}=\sum_{i} z_{i} \cdot c_{\mathrm{sp}, i}=0
$$

holds, where $\rho_{\mathrm{sp}}$ is the local charge density and $i$ runs over all ions, including $\mathrm{H}^{+}$ and $\mathrm{OH}^{-}$. Furthermore, we consider the charge balance

$$
\frac{\partial \rho_{\mathrm{sp}}}{\partial t}=-\frac{\partial}{\partial x} \sum_{i} z_{i} \cdot J_{\mathrm{sp}, i}=0
$$

in which we insert the Nernst-Planck equation, Eq. (7.1).

To evaluate the $x$-directional ionic current density, $J_{\text {charge }}$ (which is independent of $x$-position in a given sub-cell, but changes from sub-cell to sub-cell), we apply

$$
J_{\text {charge }}=\sum_{i} z_{i} \cdot J_{\mathrm{sp}, i}^{\mathrm{aux}}
$$

where again, as throughout this Chapter, the summation includes $\mathrm{Na}^{+}, \mathrm{Cl}^{-}, \mathrm{H}^{+}$ and $\mathrm{OH}^{-}$, and where $J_{\mathrm{sp}, i}^{\text {aux }}$ is given by

$$
J_{\mathrm{sp}, i}^{\mathrm{aux}}=-\frac{D_{\mathrm{eff}, i}}{L_{\mathrm{sp}}} \cdot\left(\left.c_{\mathrm{sp}, i}\right|_{\mathrm{CH} / \mathrm{M}_{\mathrm{cat}}}-\left.c_{\mathrm{sp}, i}\right|_{\mathrm{CH} / \mathrm{M}_{\mathrm{an}}}+z_{i} \int_{\left.\phi_{\mathrm{sp}}\right|_{\mathrm{CH} / \mathrm{M}_{\mathrm{an}}}}^{\left.\phi_{\mathrm{sp}}\right|_{\mathrm{CH} / \mathrm{M}_{\mathrm{cat}}}} c_{\mathrm{sp}, i} \mathrm{~d} \phi_{\mathrm{sp}}\right) .
$$

Note that $J_{\mathrm{sp}, i}^{\text {aux }}$ has no direct physical meaning and is only an auxiliary parameter. For a detailed derivation of Eq. (7.6), see 7.A.

Because we assume that all acid-base reactions are locally at equilibrium, we replace $\left[\mathrm{OH}^{-}\right]$in Eqs. (7.3)-(7.6) with $\left[\mathrm{H}^{+}\right]$using the acid-base equilibrium constant of the self-ionization reaction of water $\left(\mathrm{H}_{2} \mathrm{O} \rightleftarrows \mathrm{H}^{+}+\mathrm{OH}^{-}\right), K_{\mathrm{w}}$

$$
K_{\mathrm{w}}=\left[\mathrm{H}^{+}\right] \cdot\left[\mathrm{OH}^{-}\right]
$$

where [..] denote concentrations (used interchangeably with symbol " $c$ ").

We relate the sub-cell-dependent ionic current density to the overall average cell electric current, $I\left(\mathrm{~A} / \mathrm{m}^{2}\right)$, according to

$$
I=\frac{F}{M} \sum_{M=1}^{M} J_{\text {charge }, M}
$$

where $F$ is the Faraday constant $(96,485 \mathrm{C} / \mathrm{mol})$ and where the summation runs over all $M$ sub-cells. 


\subsubsection{Membranes}

The molar flux of ions across the membranes is again given by the Nernst-Planck equation, Eq. (7.1), where subscript "sp" is replaced by "m", and where $c_{\mathrm{m}, i}$ is the concentration of ion $i$ in the membrane, $\phi_{\mathrm{m}}$ is the potential in the membrane, and $x$ runs from the $\mathrm{CH} / \mathrm{M}$ interface to the membrane-electrode interface, $\mathrm{M} / \mathrm{E}$. Parameter $D_{\mathrm{eff}, i}$ is the effective diffusion coefficient of ion $i$ in the membrane pores, which is given by $D_{\mathrm{eff}, i}=d_{\mathrm{r}, \mathrm{m}} \cdot D_{i}$, where $d_{\mathrm{r}, \mathrm{m}}$ is a factor to reduce the diffusion coefficient in the membrane relative to the value in free solution, $D$. To evaluate ion concentrations, we set up a mass balance

$$
\frac{\partial c_{\mathrm{m}, \mathrm{i}}}{\partial t}=-\frac{\partial J_{\mathrm{m}, \mathrm{i}}}{\partial x}
$$

which we solve for $\mathrm{Na}^{+}$and $\mathrm{Cl}^{-}$. To simplify, we model steady-state ion transport across the membranes. Therefore, we can set the left-hand side of Eq. (7.9) to zero.

At each position in the membranes, we apply the electroneutrality condition

$$
\omega X+\sum_{i} z_{i} \cdot c_{\mathrm{m}, i}=0
$$

where $i$ runs over all ions, including $\mathrm{H}^{+}$and $\mathrm{OH}^{-}$, and where $X$ is the fixed membrane charge density defined per unit pore volume, and $\omega$ is the sign thereof, which is -1 for a CEM and +1 for an AEM. Furthermore, we consider the charge balance at each position in the membranes as well, given by Eq. (7.4), replacing subscript "sp" by "m".

On the spacer channel-membrane interface, and on the membrane-electrode interface, we apply

$$
\begin{array}{rr}
\left.c_{\mathrm{m}, i}\right|_{\mathrm{CH} / \mathrm{M}} & =\left.c_{\mathrm{sp}}\right|_{\mathrm{CH} / \mathrm{M}} \cdot \exp \left(-z_{i} \cdot \Delta \phi_{\mathrm{D}_{\mathrm{CH} / \mathrm{M}}}\right) \\
\left.c_{\mathrm{m}, i}\right|_{\mathrm{M} / \mathrm{E}} & =\left.c_{\mathrm{sp}}\right|_{\mathrm{M} / \mathrm{E}} \cdot \exp \left(-z_{i} \cdot \Delta \phi_{\mathrm{D}_{\mathrm{M} / \mathrm{E}}}\right)
\end{array}
$$

for every ion, where $\Delta \phi_{\mathrm{D}}$ is the Donnan potential, that is the potential just inside the membranes, relative to that just outside the membrane, in solution.

Furthermore, at the $\mathrm{CH} / \mathrm{M}$ boundary, we have continuity of ionic fluxes, which is for $\mathrm{Na}^{+}$and $\mathrm{Cl}^{-}$given by

$$
-\left.J_{\mathrm{sp}, i}\right|_{\mathrm{CH} / \mathrm{M}}+J_{\mathrm{m}, i}^{\mathrm{aux}}=0
$$

where molar flux $J_{\mathrm{m}}^{\mathrm{aux}}$ is given by Eq. (7.6) with subscript "sp" replaced by "m", "CH/ $\mathrm{M}_{\text {cat }}$ " by "M/E" and "CH/Man" by "CH/M". For the ionic current we evaluate

$$
J_{\text {charge }}=\sum_{i} z_{i} \cdot J_{\mathrm{m}, i}^{\mathrm{aux}}
$$


where $i$ runs over all ions. Note that for $\mathrm{Na}^{+}$and $\mathrm{Cl}^{-} J_{\mathrm{m}, i}^{\text {aux }}$ is the molar flux through the membrane.

In Eqs. (7.10)-(7.13) we replace $\left[\mathrm{OH}^{-}\right]$with $\frac{K_{\mathrm{w}}}{\left[\mathrm{H}^{+}\right]}$, using Eq. (7.7).

\subsubsection{Porous carbon electrodes}

We model two different types of pores in the electrodes: macropores and micropores. The macropores serve as transport pathways for ions from the $\mathrm{M} / \mathrm{E}$ boundary to the micropores, where ions are adsorbed. We distinguish two micropore regions in the model, one with a negative chemical surface charge due to the presence of acidic groups, and one with a positive surface charge due to basic groups on the carbon surface.

As transport of ions from the spacer channel, through the membranes, into the electrodes, has been found to be rate-limited by the membranes, see Chapter 3, we can assume that strong concentration gradients will not develop across the electrodes and we can consider averaged micro- and macropore concentrations (within a given sub-cell, independent of $x$-position in electrode). To model adsorption of $\mathrm{Na}^{+}$and $\mathrm{Cl}^{-}$ions in the electrodes, we evaluate the mass balance

$$
\frac{\partial}{\partial t}\left(p_{\mathrm{mA}} \cdot c_{\mathrm{mA}, i}+p_{\mathrm{mi}} \sum_{R} \alpha_{\mathrm{mi}, R} \cdot c_{\mathrm{mi}, i, R}\right)-\frac{1}{L_{\mathrm{el}}} \cdot J_{\mathrm{m}, i}^{\mathrm{aux}}=0
$$

with $p_{\mathrm{mi}}$ and $p_{\mathrm{mA}}$ the micro- and macroporosity, $c_{\mathrm{mA}, i}$ the concentration of ion $i$ in the macropores, and $L_{\mathrm{el}}$ the thickness of the electrode. Concentration $c_{\mathrm{mi}, i, R}$ is the concentration of an ion in micropore region $R$, where $R$ is either $A$ or $B, A$ for the region with acidic groups, and $B$ for the region with basic groups. The fraction of all micropore volume occupied by region $R$ is $\alpha_{\mathrm{mi}, R}$, thus $\sum_{R=\mathrm{A}, \mathrm{B}} \alpha_{\mathrm{mi}, R}=1$. In this Chapter, we set $\alpha_{\mathrm{mi}, \mathrm{A}}=\alpha_{\mathrm{mi}, \mathrm{B}}=0.5$, for both anode and cathode. For $\mathrm{Na}^{+}$and $\mathrm{Cl}^{-}$, the molar flux $J_{\mathrm{m}, i}^{\text {aux }}$ is equal at the M/E boundary to the molar flux at the $\mathrm{CH} / \mathrm{M}$ boundary, as ion transport in steady state is assumed across the membranes.

For the macropores, we apply the electroneutrality condition

$$
\sum_{i} z_{i} \cdot c_{\mathrm{mA}, i}=0
$$

The concentration of ions in each of the micropore regions, $c_{\mathrm{mi}, i, R}$, is given by

$$
c_{\mathrm{mi}, i, R}=c_{\mathrm{mA}, i} \cdot \exp \left(-z_{i} \cdot \Delta \phi_{\mathrm{D}, R}\right)
$$

where $\Delta \phi_{\mathrm{D}, R}$ is the Donnan potential, which is different for acidic and basic regions, and also different between the electrodes. 
In the micropores, for both regions separately, we evaluate the Stern potential

$$
\Delta \phi_{\mathrm{S}, R}=\frac{\sigma_{\mathrm{elec}, R} \cdot F}{V_{\mathrm{T}} \cdot C_{\mathrm{S}}}
$$

where $C_{\mathrm{S}}$ is the Stern capacitance in $\mathrm{F} / \mathrm{m}^{3}, \sigma_{\text {elec, } R}$ the electronic charge in $\mathrm{mol} / \mathrm{m}^{3}$, and $V_{\mathrm{T}}$ the thermal voltage given by $V_{\mathrm{T}}=\frac{R \cdot T}{F}$, where $R$ is the gas constant $(8.314 \mathrm{~J} /(\mathrm{mol} \mathrm{K}))$ and $T$ temperature. Both for the acidic and basic regions, the summation of ionic $\sigma_{\text {ionic, } R}$, chemical $\sigma_{\text {chem, } R}$, and electronic charge equals zero,

$$
\sigma_{\text {ionic }, R}+\sigma_{\text {chem }, R}+\sigma_{\text {elec }, R}=0
$$

where

$$
\sigma_{\text {ionic }, R}=\sum_{i} z_{i} \cdot c_{\mathrm{mi}, i, R}
$$

The potential over the EDL, $\Delta \phi_{\mathrm{EDL}}$, that is the summation of the Donnan and Stern potential, is equal for acidic and basic regions, in a given sub-cell, in a given electrode

$$
\Delta \phi_{\mathrm{EDL}}=\left(\Delta \phi_{\mathrm{D}}+\Delta \phi_{\mathrm{S}}\right)_{\mathrm{B}}=\left(\Delta \phi_{\mathrm{D}}+\Delta \phi_{\mathrm{S}}\right)_{\mathrm{A}} .
$$

To calculate the faradaic current, or reaction rate, of a faradaic reaction that takes place in the micropores, $J_{\mathrm{F}, r}$, we employ the FBV equation, which is for a one-electron reaction in general form given by

$$
J_{\mathrm{F}, r}=k_{\mathrm{R}} \cdot c_{\mathrm{O}} \cdot \exp \left(-1 / 2 \cdot \Delta \phi_{\mathrm{S}}\right)-k_{\mathrm{O}} \cdot c_{\mathrm{R}} \cdot \exp \left(+1 / 2 \cdot \Delta \phi_{\mathrm{S}}\right)
$$

where $k_{\mathrm{R}}$ is the kinetic rate constant for the reduction reaction, and $k_{\mathrm{O}}$ for the oxidation reaction; $c_{\mathrm{O}}$ is the concentration of a certain species in oxidized form, and $c_{\mathrm{R}}$ is the concentration of the reduced form, both to be evaluated at the Stern plane, which according to the Donnan model is equal to the concentration in the micropores.

In literature several faradaic reactions are reported that can occur in MCDI, such as:

- the reduction of oxygen $\left(\mathrm{O}_{2}+4 \mathrm{H}^{+}+4 \mathrm{e}^{-} \rightleftarrows 2 \mathrm{H}_{2} \mathrm{O}\right)$;

- the reduction of water $\left(2 \mathrm{H}_{2} \mathrm{O}+2 \mathrm{e}^{-} \rightleftarrows \mathrm{H}_{2}(\mathrm{~g})+2 \mathrm{OH}^{-}\right)$;

- the oxidation of carbon $\left(\mathrm{C}+\mathrm{H}_{2} \mathrm{O} \rightleftarrows \mathrm{CO}_{2}(\mathrm{~g})+4 \mathrm{H}^{+}+4 \mathrm{e}^{-}\right)$.

These reactions can be included in our model using the FBV equation, but each reaction requires values for $k_{\mathrm{R}}$ and $k_{\mathrm{O}}$, which have to be found in literature, or have to be measured. In the present Chapter, however, we aim to show the effect of faradaic reactions on $\mathrm{pH}$ fluctuations in general, and therefore we only consider the reduction of water, $2 \mathrm{H}_{2} \mathrm{O}+2 \mathrm{e}^{-} \rightleftarrows \mathrm{H}_{2}(\mathrm{~g})+2 \mathrm{OH}^{-}$, or in 
different form $1 \mathrm{H}^{+}+1 \mathrm{e}^{-} \rightleftarrows 1 / 2 \mathrm{H}_{2}(\mathrm{~g})$ (note that these reaction equations are mathematically identical in our model, because we assume that the water selfionization reaction is locally at equilibrium). We assume that the produced hydrogen gas immediately disappears from the system, i.e. the concentration of hydrogen in solution is zero (so the value of $c_{\mathrm{R}}$ is zero). Furthermore, we consider that this reaction occurs in both acidic and basic micropore regions, and thus Eq. (7.21) becomes

$$
J_{\mathrm{F}}=k_{\mathrm{R}} \sum_{R} \alpha_{\mathrm{mi}, R}\left(c_{\mathrm{mi}, \mathrm{H}^{+}, R} \cdot \exp \left(-1 / 2 \cdot \Delta \phi_{\mathrm{S}, R}\right)\right)
$$

Furthermore, we evaluate the electronic charge in both electrodes as function of the ionic current density, $J_{\text {charge }}$, and the faradaic rate, $J_{\mathrm{F}}$, according to

$$
p_{\mathrm{mi}} \cdot \frac{\partial}{\partial t} \sum_{R}\left(\alpha_{\mathrm{mi}, R} \cdot \sigma_{\mathrm{elec}, R}\right)=\frac{1}{L_{\mathrm{el}}}\left(z_{E} \cdot J_{\text {charge }}+J_{\mathrm{F}}\right)
$$

where $\sigma_{\text {elec }}$ for anode and cathode have opposite sign, i.e., $z_{E}=+1$ for the anode, and $z_{E}=-1$ for the cathode.

Now, we insert the equilibrium condition of water self-ionization, see Eq. (7.7), into Eqs. (7.15), (7.19) and (7.22) to arrive at an equation that does not explicitly include the concentration of $\mathrm{OH}^{-}$ions.

We relate Donnan and Stern potentials in the electrodes, the potential drop over the membranes, $\Delta \phi_{\mathrm{m}}$, the Donnan potentials at the membrane interfaces, and the potential drop over the spacer channel, $\Delta \phi_{\mathrm{sp}}$, to the cell voltage, $V_{\text {cell }}$, according to

$$
\frac{V_{\text {cell }}}{V_{\mathrm{T}}}=\sum_{E} z_{E}\left(\Delta \phi_{\mathrm{EDL}}+\Delta \phi_{\mathrm{m}}+\left.\Delta \phi_{\mathrm{D}}\right|_{\mathrm{CH} / \mathrm{M}}-\left.\Delta \phi_{D}\right|_{\mathrm{M} / \mathrm{E}}\right)-\Delta \phi_{\mathrm{sp}}
$$

where $E$ runs over both electrodes. Please note that $\left.\Delta \phi_{D}\right|_{\mathrm{M} / \mathrm{E}}$ and $\left.\Delta \phi_{\mathrm{D}}\right|_{\mathrm{CH} / \mathrm{M}}$ have opposite sign, as the Donnan potential is defined as a difference between the potential in the smaller pores and in the bigger pores (or open space), so in this case between the membrane and spacer channel, and between the membrane and macropores.

\subsection{Results and discussion}

To illustrate $\mathrm{pH}$ differences between feed water and effluent for MCDI predicted by theory, we first present results of equilibrium calculations under no-flow conditions, for which the water flow rate through the cell, $\Phi_{\mathrm{v}}$, is 0 . Thereafter, we present dynamic calculations with flow. 


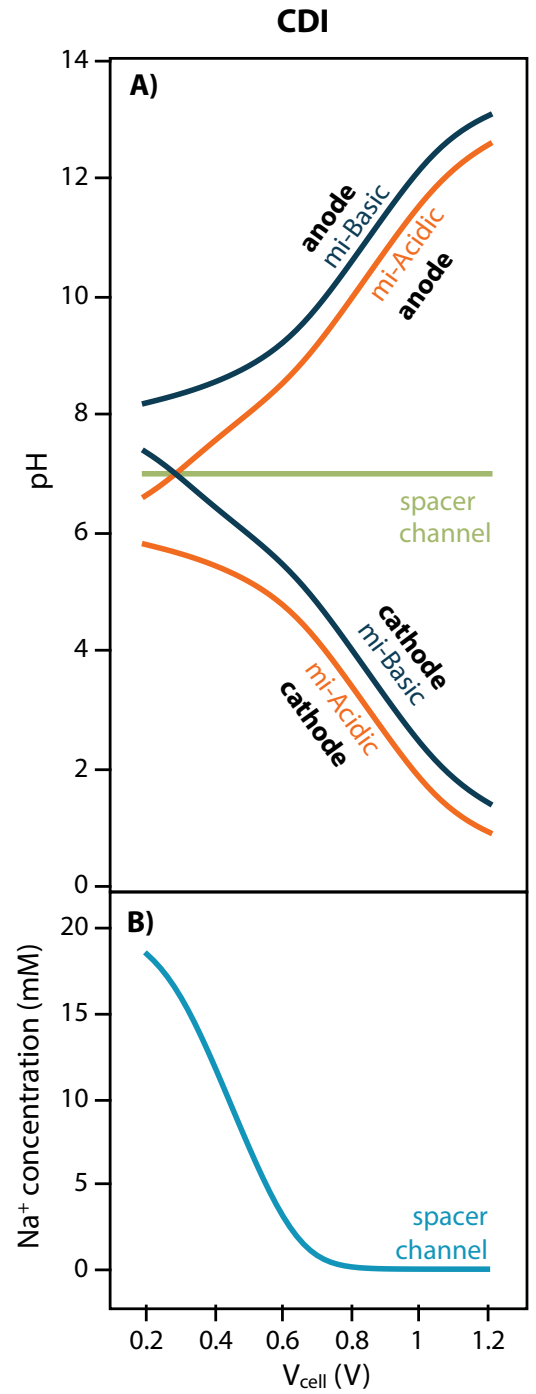

Fig. 7.2: Equilibrium calculations (no-flow) of $\mathrm{pH}$ and $\mathrm{Na}^{+}$-concentration as function of cell voltage, $V_{\text {cell }}$, in a CDI cell. $\mathrm{pH}$ in the spacer channel and in acidic (mi-Acidic) and basic (mi-Basic) micropore regions are shown, for anode and cathode. 


\subsubsection{Equilibrium calculations}

For no-flow conditions, we calculated the equilibrium $\mathrm{pH}$ and salt concentration in the CDI cell as function of the cell voltage, see Fig. $7.2^{1}$. We excluded faradaic reactions from these calculations, thus we used $k_{\mathrm{R}}=0$ in Eq. (7.22). The initial $\mathrm{pH}$ of the solution in the spacer channel was $\mathrm{pH} 7$, and the initial salt concentration was $20 \mathrm{mM}$.

Fig. 7.2A shows that $\mathrm{pH}$ in the spacer channel is, in equilibrium, equal to the initial pH. Furthermore, Fig. 7.2A shows, as function of the cell voltage, an increase of $\mathrm{pH}$ in both micropore regions in the anode and a decrease in the cathode. $\mathrm{pH}$ and $\mathrm{Na}^{+}$-concentration (see Fig. 7.2B) in the macropores of the electrodes is equal to $\mathrm{pH}$ in the spacer channel, and is not plotted.

\subsubsection{Dynamic calculations}

For flow conditions, we performed calculations for an MCDI cell, which we charged with a constant current of $20 \mathrm{~A} / \mathrm{m}^{2}$ until we reached a cell voltage of $1.2 \mathrm{~V}$, and thereafter discharged with the reversed current until a voltage of $0 \mathrm{~V}$. The initial $\mathrm{pH}$ of the solution in the spacer channel was $\mathrm{pH} 7$, and the initial salt concentration was $20 \mathrm{mM}$. We calculated two cases: "Only Capacitive" (OC) and "Capacitive and Faradaic" (CF). For the OC-case we excluded faradaic reactions from the calculations $\left(k_{\mathrm{R}}=0\right)$.

For the OC-case, Fig. 7.3 column I) shows that $\mathrm{pH}$ in the spacer channel, which is equal to the effluent $\mathrm{pH}$, increases during charging and decreases during discharge, but that these $\mathrm{pH}$ changes are very small compared to the $\mathrm{pH}$ changes in anode and cathode. In the anode, the model predicts an increase of $\mathrm{pH}$ during charging and a decrease during discharge, in both macro- and micropores. In the cathode, however, the model predicts a decrease during charging and an increase during discharge. The $\mathrm{pH}$ profile across the spacer channel, in the spacer between AEM and CEM, is shown in Fig. 7.4.

For the CF-case, Fig. 7.3 column II) shows that changes in effluent $\mathrm{pH}$ as function of time are much stronger than for the OC-case. Clearly, including the water reduction reaction, $2 \mathrm{H}_{2} \mathrm{O}+2 \mathrm{e}^{-} \longrightarrow \mathrm{H}_{2}(\mathrm{~g})+2 \mathrm{OH}^{-}$, results in an effluent $\mathrm{pH}$ which is higher, during charging and discharge, than the inflow $\mathrm{pH}$. These calculation results are similar to results of lab-scale experiments, which are shown in Fig. $7.5^{2}$. The results shown in Fig. 7.3 column II) are strongly dependent

\footnotetext{
${ }^{1}$ The CDI equilibrium state was calculated with a simplified version of the theory described in Section 7.2; we excluded the membranes and set all terms with a derivative to time to zero.

${ }^{2}$ The materials and methods of the experiments shown in Fig. 7.5 are described in Appendix 7.B
} 
I) Only Capacitive

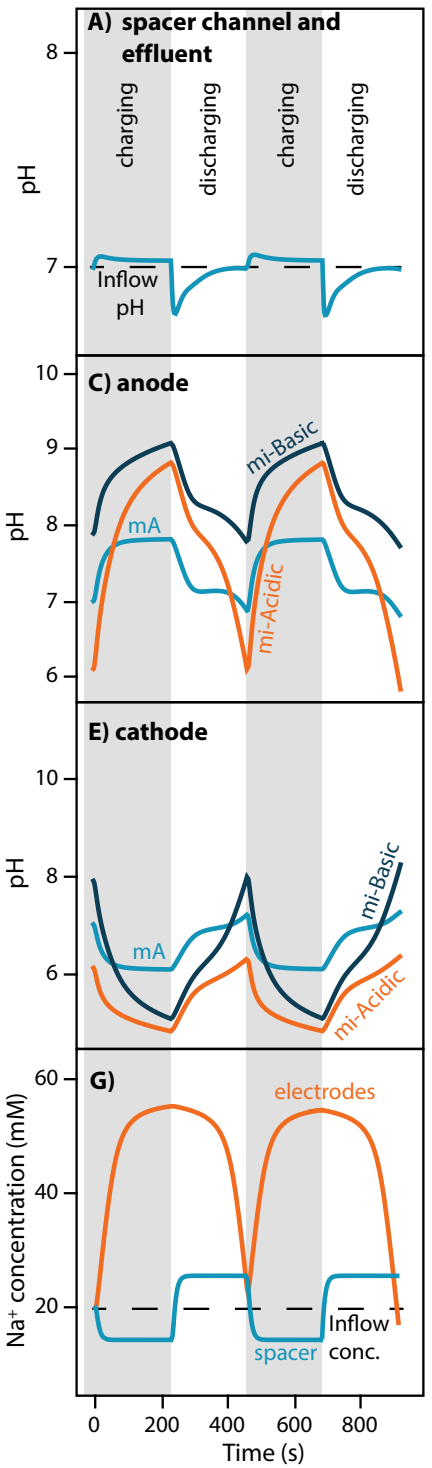

II) Capacitive and Faradaic

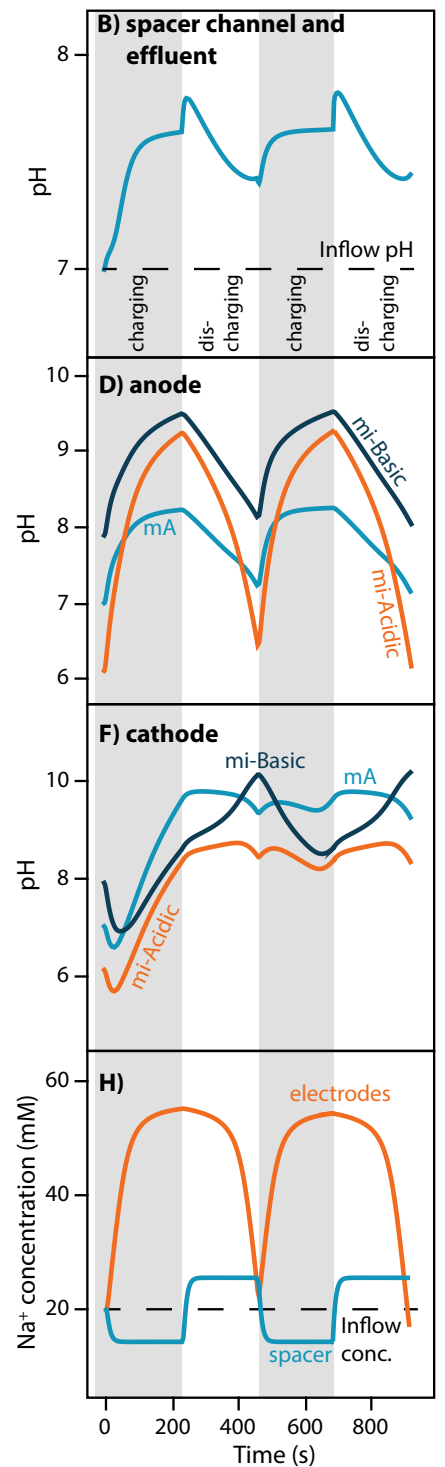

Fig. 7.3: Dynamic calculations (with flow) of $\mathrm{pH}$ and $\mathrm{Na}^{+}$-concentration as function of time in an MCDI cell, for cases "Only Capacitive" (column I) and "Capacitive and Faradaic" (column II). The cell was charged with a constant current of $20 \mathrm{~A} / \mathrm{m}^{2}$ until a cell voltage of $1.2 \mathrm{~V}$ was reached, and thereafter discharged with a reversed current until a voltage of $0 \mathrm{~V} . \mathrm{pH}$ in the spacer channel, equal to effluent $\mathrm{pH}$, is shown (A and $\mathrm{B}$ ), as well as the $\mathrm{pH}$ in the acidic (mi-Acidic) and basic (mi-Basic) micropore regions, both for anode $(\mathrm{C}$ and $\mathrm{D})$ and cathode $(\mathrm{E}$ and $\mathrm{F}) \cdot \mathrm{Na}^{+}$-concentrations in anode and cathode are equal to each other, with a value very close to the $\mathrm{Cl}^{-}$-concentrations $(\mathrm{G}$ and $\mathrm{H})$. 


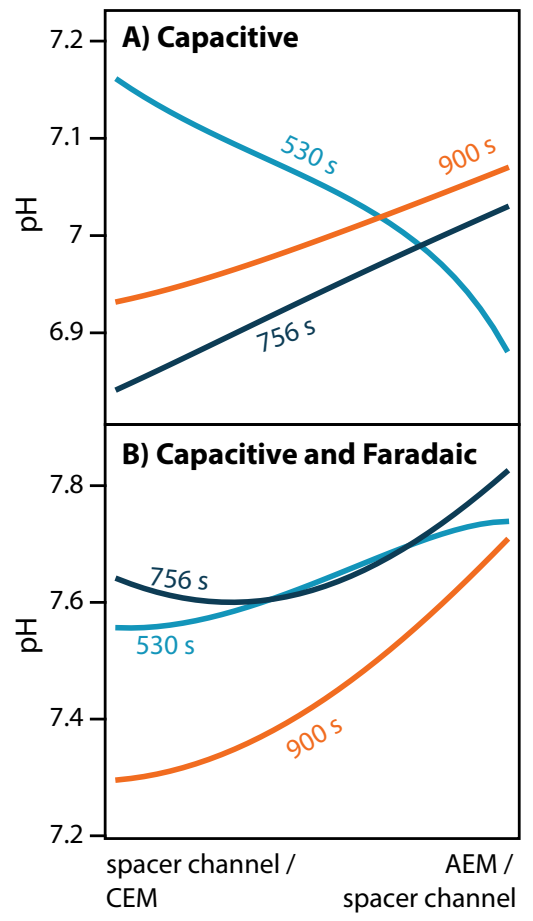

Fig. 7.4: $\mathrm{pH}$ profiles across the spacer channel based on calculations under MCDI flow conditions, both for the case "Only Capacitive" (A) and the case "Capacitive and Faradaic" (B), at different moments during a desalination cycle as shown in Fig. 7.3.

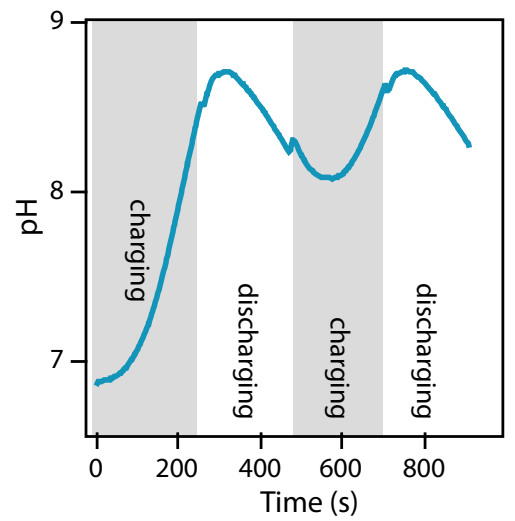

Fig. 7.5: Experimental data of effluent $\mathrm{pH}$ as function of time of an MCDI experiment conducted under conditions comparable to model conditions described for Fig. 7.3. For a detailed description of experimental conditions, see Appendix 7.B. 


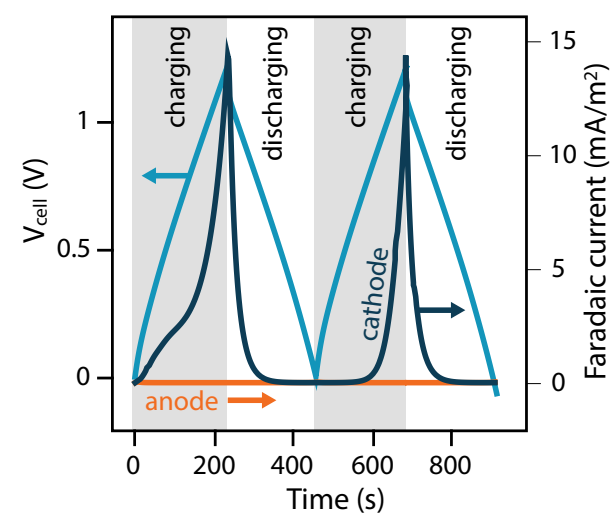

Fig. 7.6: Cell voltage and faradaic current at anode and cathode as function of time for the case "Capacitive and Faradaic" shown in Fig. 7.3.

on the value of $k_{\mathrm{R}}$, which we chose arbitrarily and is not based on the results of experimental work.

Fig. 7.6 shows that the faradaic current, and consequently hydroxyl ion production, occurs mainly in the cathode, and that this reaction does not occur in the anode. Obviously, $\mathrm{pH}$ in the cathode is higher for the CF-case than for the OC-case, during the complete cycle, see Fig. 7.3F. We note that using Eq. (7.22) the water reduction reaction cannot run for infinite time in our calculation, as we do not consider an oxidation reaction. This leads, in our calculations, to an ongoing drain of electrons from the system, which is physically not possible. Our calculation results, however, are not for a limit cycle, but for the first cycle.

We showed that the OC-case does not predict the strong $\mathrm{pH}$ changes observed experimentally, but that the CF-case does. A quantitative comparison between data and theory is not possible yet, as more faradaic reactions should then be considered in the model, and values for the rate constants of these reactions have to be determined. 
Table 7.1: System and electrode dimensions, operational parameters, and parameters used for theoretical calculations. Parameters obtained from *1) Chapter 3 ; *2) Ref. [143]; *3) Ref. [190]

\begin{tabular}{|c|c|c|c|c|}
\hline \multicolumn{5}{|c|}{ General parameters } \\
\hline$A_{\text {cell }}$ & \multicolumn{2}{|c|}{ Electrode geometric surface area } & 33.8 & $\mathrm{~cm}^{2}$ \\
\hline$\Phi_{\mathrm{v}}$ & \multicolumn{2}{|c|}{ Water flowrate through one cell } & 7.5 & $\mathrm{~mL} / \mathrm{min}$ \\
\hline$T$ & \multicolumn{2}{|c|}{ Temperature } & 295 & $\mathrm{~K}$ \\
\hline$L_{\mathrm{sp}}$ & \multicolumn{2}{|c|}{ Thickness spacer channel } & 300 & $\mu \mathrm{m}$ \\
\hline $\mathrm{pH}_{\text {inflow }}$ & \multicolumn{2}{|c|}{ Inflow $\mathrm{pH}$} & 7 & \\
\hline$M$ & \multicolumn{2}{|c|}{ Number of mathematical sub-cells } & 1 & \\
\hline \multicolumn{5}{|c|}{ Electrode parameters } \\
\hline$L_{\mathrm{el}}$ & \multicolumn{2}{|c|}{ Electrode thickness } & 260 & $\mu \mathrm{m}$ \\
\hline$v_{\mathrm{mi}}$ & \multicolumn{2}{|c|}{ Micropore volume ${ }^{* 1}$} & 0.40 & $\mathrm{~mL} / \mathrm{g}$ \\
\hline$C_{\mathrm{S}}$ & \multicolumn{2}{|c|}{ Stern capacitance ${ }^{* 1}$} & 160 & $\mathrm{~F} / \mathrm{mL}$ \\
\hline$k_{\mathrm{R}}$ & \multicolumn{2}{|c|}{ Kinetic rate constant reduction reaction } & 0.02 & $\mathrm{~m} / \mathrm{s}$ \\
\hline$\sigma_{\text {chem,A }}$ & \multicolumn{2}{|c|}{$\begin{array}{l}\text { Chemical surface charge acidic region } \\
\text { (both anode and cathode) }\end{array}$} & -240 & $\mathrm{mM}$ \\
\hline$\sigma_{\text {chem,B }}$ & \multicolumn{2}{|c|}{$\begin{array}{l}\text { Chemical surface charge basic region } \\
\text { (both anode and cathode) }\end{array}$} & 240 & $\mathrm{mM}$ \\
\hline$\alpha_{\mathrm{mi}, \mathrm{A}}=\alpha_{\mathrm{mi}, \mathrm{B}}$ & \multicolumn{2}{|c|}{$\begin{array}{l}\text { Volume ratio acidic and basic } \\
\text { regions micropores }\end{array}$} & $1: 1$ & \\
\hline$p_{\mathrm{mA}}$ & \multicolumn{2}{|c|}{ Macroporosity $^{* 1}$} & 0.48 & \\
\hline$p_{\mathrm{mi}}$ & \multicolumn{2}{|c|}{ Microporosity ${ }^{* 1}$} & 0.23 & \\
\hline$p_{\mathrm{sk}}$ & \multicolumn{2}{|c|}{ Fraction "skeleton" material electrode ${ }^{* 1}$} & 0.29 & \\
\hline \multicolumn{5}{|c|}{ Membrane parameters } \\
\hline$L_{\mathrm{m}, \mathrm{AEM}}$ & \multicolumn{2}{|c|}{ AEM thickness } & 130 & $\mu \mathrm{m}$ \\
\hline$L_{\mathrm{m}, \mathrm{CEM}}$ & \multicolumn{2}{|c|}{ CEM thickness } & 190 & $\mu \mathrm{m}$ \\
\hline$d_{\mathrm{r}, \mathrm{m}}$ & \multicolumn{2}{|c|}{$\begin{array}{l}\text { Diffusion coefficient in membrane pores } \\
\text { relative to value in free solution }\end{array}$} & 0.05 & \\
\hline$\omega_{\mathrm{AEM}}$ & \multicolumn{2}{|c|}{ Sign of the fixed charge AEM } & +1 & \\
\hline$\omega_{\mathrm{CEM}}$ & \multicolumn{2}{|c|}{ Sign of the fixed charge CEM } & -1 & \\
\hline$X_{\mathrm{AEM}}$ & \multicolumn{2}{|c|}{ Fixed charge density AEM } & 5 & M \\
\hline$X_{\mathrm{CEM}}$ & \multicolumn{2}{|c|}{ Fixed charge density CEM } & 5 & M \\
\hline \\
\hline \multicolumn{2}{|c|}{ Diffusion coefficients and inflow concentrations ${ }^{*}{ }_{3}$} & $c_{\text {inflow }, i}$ & $D_{i}^{* 3}$ & \\
\hline $\mathrm{Na}^{+}$ & Sodium & $20.0 \mathrm{mM}$ & 1.33 & $\cdot 10^{-9} \mathrm{~m}^{2} / \mathrm{s}$ \\
\hline $\mathrm{Cl}^{-}$ & Chloride & $20.0 \mathrm{mM}$ & 2.02 & $\cdot 10^{-9} \mathrm{~m}^{2} / \mathrm{s}$ \\
\hline $\mathrm{H}^{+}$ & Proton & $\mathrm{mM}$ & 9.13 & $\cdot 10^{-9} \mathrm{~m}^{2} / \mathrm{s}$ \\
\hline $\mathrm{OH}^{-}$ & Hydroxyl ion & $10^{-4} \mathrm{mM}$ & 5.16 & $\cdot 10^{-9} \mathrm{~m}^{2} / \mathrm{s}$ \\
\hline \multicolumn{5}{|c|}{$\begin{array}{l}\text { Listed inflow concentrations are calculated considering conditions } \\
\text { given by Eqs. }(7.3) \text { and }(7.7) \text {, and } \mathrm{pH}_{\text {inflow }}=3-\log _{10}\left(c_{\text {inflow }, \mathrm{H}^{+}}\right) \text {. }\end{array}$} \\
\hline & Equilibrium & constant of water self-ioniz & & \\
\hline $\begin{array}{l}\mathrm{pK}_{\mathrm{w}} \\
\mathrm{pK}_{\mathrm{w}} \text { relates to }\end{array}$ & $\begin{array}{c}\mathrm{H}_{2} \mathrm{O} \underset{\mathrm{H}^{+}}{\rightleftarrows} \\
K_{\mathrm{w}} \text { using } \log _{10}\end{array}$ & $\begin{array}{l}+\mathrm{OH}^{-}, K_{\mathrm{w}}=\left[\mathrm{H}^{+}\right]\left[\mathrm{OH}^{-}\right] \\
\left.K_{\mathrm{w}}\right)=6-\mathrm{pK}_{\mathrm{w}}\end{array}$ & 14.00 & \\
\hline
\end{tabular}




\subsection{Conclusions}

We presented theory to calculate $\mathrm{pH}$ changes in electrochemical systems for water desalination, which we used to predict $\mathrm{pH}$ changes and differences in $\mathrm{pH}$ between feed water and effluent observed in Membrane Capacitive Deionization. In our theory, we included three phenomena that occur in MCDI that may explain the observed pH changes: I) different mobilities of various ions; II) chemical surface charge groups in the micropores of the porous carbon electrodes; and III) faradaic reactions in the micropores.

Our theory predicts small pH changes during desalination cycles in MCDI if we only consider phenomena I) and II). If we also consider phenomenon III) by including the water reduction reaction $2 \mathrm{H}_{2} \mathrm{O}+2 \mathrm{e}^{-} \longrightarrow \mathrm{H}_{2}(\mathrm{~g})+2 \mathrm{OH}^{-}$, pH changes are stronger. We see that theory predicts an increase of $\mathrm{pH}$ during charging and a decrease during discharge, which is also observed in our experiments. 


\section{Appendix}

\section{A Derivation of auxiliary flux $J_{j, i}^{\text {aux }}$}

In the main text, the theory for spacer channel and membranes makes use of the "auxiliary" flux $J_{j, i}^{\text {aux }}$, a flux-like expression which only in a few instances has a physical meaning. The expression for $J_{j, i}^{\text {aux }}$ is given for the spacer channel by Eq. (7.6) in the main text $(j=\mathrm{sp})$. For the two membranes $(j=\mathrm{m})$, subscripts "sp" must be replaced by "m" in Eq. (7.6). The integration boundaries are then the two outer boundaries of the membrane.

For the spacer channel, Eq. (7.6) is also used to calculate the current density, $J_{\text {charge, }}$, while for each membrane, Eq. (7.6) is used to calculate the flux of $\mathrm{Na}^{+}$ and $\mathrm{Cl}^{-}$. All these fluxes (currents) are invariant with coordinate $x$.

Here we derive Eq. (7.6) on the basis of transport across the membrane. The membrane is described in steady state, thus all time-dependencies vanish. For each ion, the molar flux through the membrane, $J_{\mathrm{m}, i}$, is given by the NernstPlanck equation

$$
J_{\mathrm{m}, i}=-D_{\mathrm{eff}, i}\left(\frac{\partial c_{\mathrm{m}, i}}{\partial x}+z_{i} \cdot c_{\mathrm{m}, i} \cdot \frac{\partial \phi_{\mathrm{m}}}{\partial x}\right)
$$

where $c_{\mathrm{m}, i}$ is the concentration of ion $i$ in the membrane, defined per unit open volume (per volume of pore), $z_{i}$ is the valence of an ion, and $D_{\text {eff, } i}$ is the effective diffusion coefficient. Variable $\phi_{\mathrm{m}}$ is the dimensionless potential in the membrane, and $x$ runs from the spacer channel-membrane boundary, $\mathrm{CH} / \mathrm{M}$, to the membrane-electrode boundary, M/E. 
For $\mathrm{Na}^{+}$and $\mathrm{Cl}^{-}$, which are unreactive, and because we model steady state, ion transport $J_{\mathrm{m}, i}$ is constant across the membranes, i.e., $J_{\mathrm{m}, i}$ is independent of $x$. Therefore, we can integrate Eq. (7.25) to

$$
\int_{0}^{L_{\mathrm{m}}} J_{\mathrm{m}, i} \mathrm{~d} x=-D_{\mathrm{eff}, i}\left(\int_{\left.c_{\mathrm{m}, i}\right|_{\mathrm{CH} / \mathrm{M}}}^{\left.c_{\mathrm{m}, i}\right|_{\mathrm{M} / \mathrm{E}}} \mathrm{d} c_{\mathrm{m}, i}+\int_{\left.\phi_{\mathrm{m}}\right|_{\mathrm{CH} / \mathrm{M}}}^{\left.\phi_{\mathrm{m}}\right|_{\mathrm{M} / \mathrm{E}}} z_{i} c_{\mathrm{m}, i} \mathrm{~d} \phi_{\mathrm{m}}\right)
$$

where $L_{\mathrm{m}}$ is the thickness of the membrane. As $\operatorname{lns}($ Eq. $(7.26))=J_{\mathrm{m}, i} L_{\mathrm{m}}$, we arrive at

$$
J_{\mathrm{m}, i}^{\mathrm{aux}}=-\frac{D_{\mathrm{eff}, i}}{L_{\mathrm{m}}}\left(\left.c_{\mathrm{m}, i}\right|_{\mathrm{M} / \mathrm{E}}-\left.c_{\mathrm{m}, i}\right|_{\mathrm{CH} / \mathrm{M}}+z_{i} \int_{\left.\phi_{\mathrm{m}}\right|_{\mathrm{CH} / \mathrm{M}}}^{\left.\phi_{\mathrm{m}}\right|_{\mathrm{M} / \mathrm{E}}} c_{\mathrm{m}, i} \mathrm{~d} \phi_{\mathrm{m}}\right)
$$

where superscript "aux" is introduced to denote the mathematical notation of Eq. (7.27). Besides using Eq. (7.27) for the flux of $\mathrm{Na}^{+}$and $\mathrm{Cl}^{-}$, we know that, in steady state, current $J_{\text {charge }}$ is invariant across the membrane, and thus Eq. (7.13) of the main text can be used based on Eq. (7.27) above for $J_{\mathrm{m}, i}^{\mathrm{aux}}$. Furthermore, we use Eq. (7.27) to calculate the ionic current in the spacer channel. Although flow in the spacer channel is not modelled in steady state, current is independent of position $x$ across the channel, and therefore Eq. (7.5) applies.

\section{B Experimental data - Materials and Methods}

To conduct MCDI experiments, we constructed a stack with four MCDI cells. Every cell consisted of a pair of electrodes coated on graphite current collectors, which connected the electrodes with the electric circuit. The electrodes were commercial electrodes provided by Voltea (Sassenheim, The Netherlands). A CEM (Neosepta CMX) was placed on the cathode, while an AEM (Neosepta AMX) was placed on the anode. The water flowed through a spacer (commercial meshwoven spacer) positioned between the CEM and AEM. The stack of MCDI cells was placed in a polymethylmethacrylate (PMMA) housing. For geometric and material properties of electrodes, membranes, and spacer we refer to Table 7.1.

A recirculation vessel with $10 \mathrm{~L} 20 \mathrm{mM} \mathrm{NaCl}$ solution was constantly kept at $\mathrm{pH} 7 \pm 0.05$ and purged with nitrogen, to avoid the presence of oxygen in the electrolyte. From the recirculation vessel, the solution was pumped through the MCDI cells, and thereafter the solution passed a $\mathrm{pH}$ and a conductivity sensor, which measured at a sampling time of $1 \mathrm{~s}$. The effluent conductivity was recalculated to salt concentration using a calibration table.

Of each experiment, the cell voltage or current was controlled with a potentiostat (IviumStat, Ivium Technologies, The Netherlands). For our constant current experiments, we charged the cell with a current of $20 \mathrm{~A} / \mathrm{m}^{2}$ until we reached a 
cell voltage of $1.2 \mathrm{~V}$, and thereafter, we discharged the cell with a reversed current until a cell voltage of $0 \mathrm{~V}$. Experimental data presented in this Chapter are shown for the first constant current experiments conducted with new electrode materials. 



\section{General discussion and conclusions}




\subsection{Introduction}

In this Chapter we present the findings of our work and we discuss how the work contributes to the development of Capacitive Deionization (CDI) and specifically Membrane Capacitive Deionization (MCDI).

We started this Thesis by introducing both CDI and MCDI, and explained the main advantages of MCDI compared to CDI, which we briefly summarize: whereas CDI shows that, during the adsorption step, not only counterions are adsorbed into the electrodes, but also co-ions are desorbed, which is an unwanted process, MCDI has membranes placed in front of the electrodes, that block the transport of co-ions from the electrodes into the spacer channel. Therefore, the charge efficiency, that is, the ratio of salt adsorbed over charge transferred, is higher for MCDI than for classical CDI [34-38].

In this Thesis, we reported both on classical CDI and MCDI. Nowadays, as technology providers are focused on the development of MCDI, this process is of technological interest. From a scientific point of view, also CDI is interesting, as it allows us to study ion adsorption phenomena in porous electrodes without the effect of ion exchange membranes placed in front. Findings of studies on CDI can then also contribute to the development of MCDI.

The research can be divided in three topics: I) energy consumption and resistance identification, II) ion selective adsorption, and III) long-term operation and $\mathrm{pH}$ changes. We cover these topics in the next sections. Thereafter we present an outlook on technology development.

\subsection{Energy consumption and resistance identification}

In Section 1.5 we discussed the theoretical minimum energy consumption as function of desalination, $\Delta c$, which is the average concentration difference between produced fresh water and feed water, and as function of water recovery, WR, which is the amount of produced desalinated water per unit of feed water, see Fig. 1.10. In Chapter 3 we showed that the energy consumption of desalination by CDI and MCDI is much higher than the theoretical minimum energy consumption. Furthermore, we found, for the particular cycle analyzed (cycle of an MCDI cell operated in constant current mode), a total energy consumption of 85 $\mathrm{kJ} / \mathrm{mol}$ (defined per mol of salt removed), of which only $6 \mathrm{~kJ} / \mathrm{mol}$ was attributed to ionic resistances in the electrodes, membranes and spacer channel, and electronic resistances in cables and current collectors. We discussed, but without making a quantitative analysis, that the remaining energy consumption must be due to Donnan potentials at the membrane interfaces, and due to Donnan and Stern potentials in the electrodes. Now, we make this analysis and split the total 


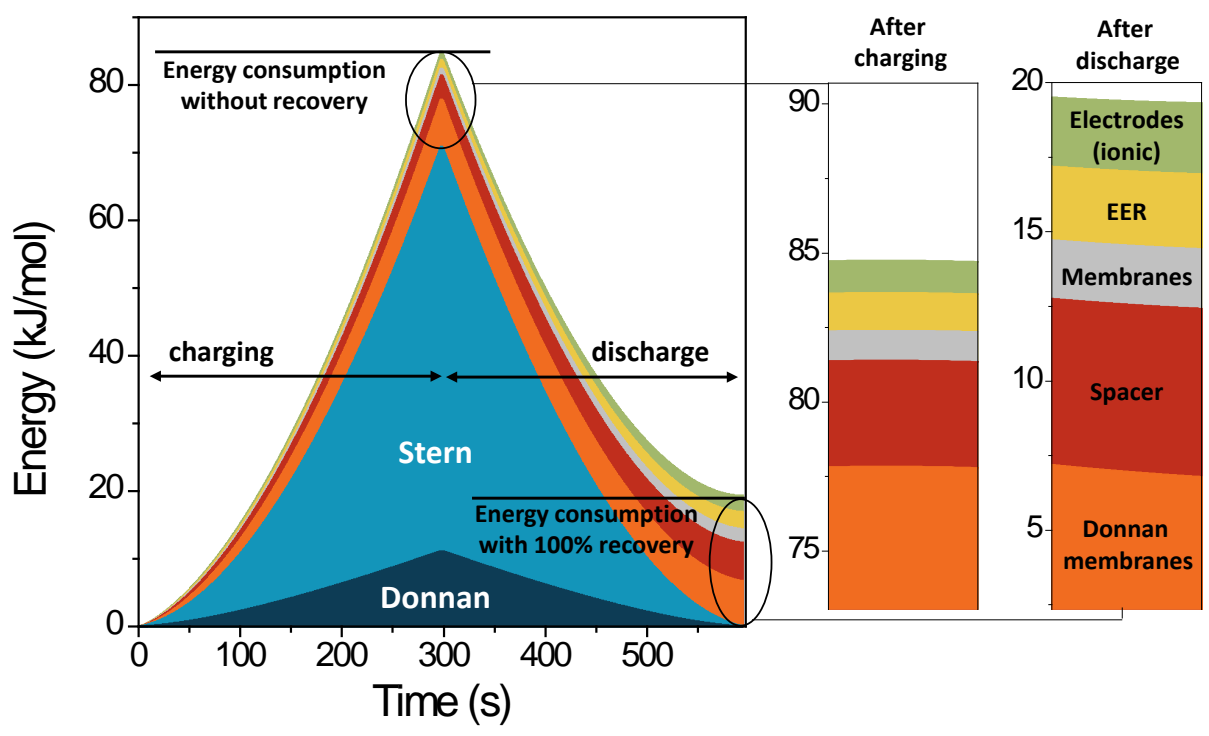

Fig. 8.1: Energy consumption during charging and energy release during discharge of an MCDI cell, calculated for a desalination cycle operated under constant current conditions presented in Section 3.5, Fig. $3.7\left(c_{\text {inflow }}=20 \mathrm{mM}\right)$. Energy consumed by charging EDLs (Donnan and Stern) can be partially recovered during discharge. Energy consumed by ionic resistances in spacer, membranes and electrodes, and by electronic resistances in cables and current collectors (EER) increases during charging and discharge. Also, energy consumed by Donnan potentials at the spacer-membrane and membrane-electrode interfaces is indicated (Donnan membranes).

energy consumption in parts. Therefore, we need an additional set of equations, see Box 8.1, to post-calculate theoretical data obtained in Chapter 3.

\section{Box 8.1: theory for energy analysis}

To split the total energy consumption in parts, we need an additional set of equations to post-calculate theoretical data presented in Chapter 3. To that end, we use the theory presented in Box 4.1 to calculate the energy consumption by EDL charging, and by dissipation due to ionic resistances in the electrodes and spacer, and due to electronic resistances in the cables and current collectors. Furthermore, we calculate the energy consumption due to dissipation by the membranes and due to the Donnan potentials at the membrane interfaces, for which we present theory below. 


\section{Ionic resistance membranes}

The energy consumption due to ionic resistances in the membrane is given by

$$
E_{\mathrm{mem}}=2 \cdot A_{\text {cell }} \cdot V_{\mathrm{T}} \int_{0}^{t}\left(\left.\Delta \phi_{\mathrm{mem}}\right|_{\mathrm{M} / \mathrm{E}}(t)-\left.\phi_{\mathrm{mem}}\right|_{\mathrm{S} / \mathrm{M}}(t)\right) \cdot I_{\mathrm{A}}(t) \mathrm{d} t
$$

where $A_{\text {cell }}$ is the electrode geometric surface area $\left(\mathrm{m}^{2}\right), I_{\mathrm{A}}(t)$ the current density $\left(\mathrm{A} / \mathrm{m}^{2}\right)$, and $V_{\mathrm{T}}$ the thermal voltage, which is given by $V_{\mathrm{T}}=R \cdot T / F$, where $R$ is the gas constant $(8.314 \mathrm{~J} /(\mathrm{mol} \cdot \mathrm{K}))$, and $T$ temperature $(\mathrm{K})$. Variable $\phi_{\text {mem }}$ is the membrane potential at the membraneelectrode interface, $M / E$, or at the spacer-membrane interface, $S / M$. A factor 2 is included to calculate $E_{\text {mem }}$ for both membranes together, which we also include in Eq. (8.2). Please note that $E_{\text {mem }}$ does not include the energy consumption term due to Donnan potentials at the membrane interfaces. This term will be calculated separately.

\section{Donnan potentials membrane interfaces}

At the spacer-membrane and membrane-electrode interfaces, Donnan potentials are considered, see Fig. 3.1. These Donnan potentials, indicated by $\Delta \phi_{\mathrm{D}}$, result in an additional energy consumption, which is given by

$$
E_{\mathrm{D}, \mathrm{mem}}=2 \cdot A_{\text {cell }} \cdot V_{\mathrm{T}} \int_{0}^{t}\left(\left.\Delta \phi_{\mathrm{D}}\right|_{\mathrm{S} / \mathrm{M}}(t)-\left.\Delta \phi_{\mathrm{D}}\right|_{\mathrm{M} / \mathrm{E}}(t)\right) \cdot I_{\mathrm{A}}(t) \mathrm{d} t .
$$

Results of the energy analysis are shown in Fig. 8.1, from which the following conclusions can be drawn:

- Energy consumption of a desalination cycle without energy recovery during discharge is $85 \mathrm{~kJ} / \mathrm{mol}$, of which only $7 \mathrm{~kJ} / \mathrm{mol}$ is lost due to electronic and ionic resistances, and $78 \mathrm{~kJ} / \mathrm{mol}$ is stored in EDLs (Donnan and Stern), which is, in theory, recoverable. We note that, in this analysis, we do not consider energy consumption due to (unwanted) faradaic reactions that may occur during desalination.

- During discharge all energy stored in EDLs is released. Part of this energy is lost due to ionic and electronic resistances, and consequently the energy recovery potential is lower than $78 \mathrm{~kJ} / \mathrm{mol}$, namely $85-19=66 \mathrm{~kJ} / \mathrm{mol}$. Thus, by employing energy recovery technology with an efficiency of $100 \%$, $77 \%$ of the energy consumption during charging can be recovered during 
discharge. We note that an energy recovery efficiency of $100 \%$ is in reality too optimistic, but efficiencies in the range of $50-80 \%$ are reported in literature $[135,156,191]$. This finding implies that, in order to reduce energy costs of desalination, developing efficient energy recovery technology will have a larger impact than reducing resistances. Only after implementing energy recovery technology, it will be interesting to focus on the reduction of resistances.

- As Fig. 8.1 shows, without energy recovery, the contribution of ionic resistances across the membranes to the total energy consumption is small $(1.2 \%)$. This implies that an eventual reduction of the membrane resistance will lower the energy consumption only to limited extent. In contrary, a reduction of the membrane resistance can even result in higher energy consumption. As a reduction of the membrane resistance is most likely achieved by a reduction of the membrane thickness, employing IEMs with lower resistances can go with a decrease in selectivity, see Fig. 1.4A. Then, the performance of membranes to block transport of co-ions from the electrodes into the spacer channel decreases, which results in a reduced charge efficiency, and thereby in increased energy consumption, see Fig. 1.4B.

- Energy consumption due to the Donnan potentials at the membrane interfaces was not considered in Chapter 3. This energy term increases during charging, when ions are concentrated in the electrodes (ion separation), and decreases during discharge (ion mixing). Admittedly, we expected that the value found for $E_{\mathrm{D} \text {,mem }}$ at the end of a discharge step would be very close to the theoretical minimum energy consumption for desalination, $\Delta G$, which is, as shown in Fig. 1.10, never more than $0.5 \mathrm{~kJ} / \mathrm{mol}$ for the cycle we analyzed. However, $E_{\mathrm{D} \text {,mem }}$ is more than a factor 15 higher. This unexpected result deserves more theoretical analysis in order to fully understand the difference between the theoretical minimum energy consumption and the real energy consumption.

- To calculate the energy consumption due to ionic resistances in the electrodes, $E_{\mathrm{mA}}$, we introduced Eq. (4.2) in Chapter 4, which is different from Eq. (3.29) used in Chapter 3 to approximate the ionic resistance across the two electrodes in an MCDI cell. In present Chapter, we apply Eq. (4.2) to post-calculate the theoretical data obtained in Chapter 4 for MCDI, and we find that the energy consumption due to ionic resistances in the electrodes $(1.1 \mathrm{~kJ} / \mathrm{mol})$ is much higher than the value following from Fig. 3.7D $(0.04$ $\mathrm{kJ} / \mathrm{mol}$ ), which was calculated using Eq. (3.29). Clearly, the assumption behind Eq. (3.29) was not valid. However, the conclusion drawn in Chap- 
ter 3 that the contribution of the ionic resistance in the electrodes to the total energy consumption is very small, does still hold.

\subsection{Ion selective adsorption}

In Chapter 5, we extended the theory of Chapter 3 to calculate the transport and adsorption of one monovalent salt, such as $\mathrm{KCl}$ or $\mathrm{NaCl}$, to mixtures of monovalent salts. We used this extended theory to model the dynamics of ion adsorption of a solution containing $\mathrm{NaCl}$ and $\mathrm{KCl}$, considering different diffusion coefficients of ions present in solution. We compared theory with experimental data of ion adsorption as function of the cycle time (CT), which is the total duration of both the charging and discharge step.

We observed, experimentally, a preferential adsorption of $\mathrm{K}^{+}$over $\mathrm{Na}^{+}$ions, which is in line with theory for short CTs. At longer CTs, however, we observe experimentally still a preferential adsorption of $\mathrm{K}^{+}$over $\mathrm{Na}^{+}$, but theory predicts that the adsorption of both ions becomes equal. This prediction is due to the fact that our dynamic theory reduces to the equilibrium improved modified Donnan (i-mD) model for long CTs, a model which, by itself, does not account for a different repulsion or attraction of one ion over another, unless the ions have different charge.

The preferential adsorption of $\mathrm{K}^{+}$over $\mathrm{Na}^{+}$, or more general, of smaller over bigger ions, also in equilibrium conditions, has been reported in other work as well, see Refs. [32, 96, 189]. In the EDL models used in this work, the i-mD and amphoteric Donnan (amph-D) model, it is assumed that ions are volume-less points, and selectivity based on size is thus not included [32]. Suss describes in Ref. [32] how the preferential adsorption based on ion-size can be considered, namely by adding an excess chemical potential difference between micro- and macropores, $\Delta \mu_{i}^{\text {ex }}$, to Eq. (2.1) for the i-mD or Eq. (7.16) for the amph-D model. Considering the amph-D model, Eq. (7.16), which relates the ion concentration in micro- and macropores, becomes

$$
c_{\mathrm{mi}, i}=c_{\mathrm{mA}, i} \cdot \exp \left(-z_{i} \cdot \Delta \phi_{\mathrm{D}}-\Delta \mu_{i}^{\mathrm{ex}}\right)
$$

where $c_{\mathrm{mi}, i}$ is the concentration of ion $i$ in the micropores, $c_{\mathrm{mA}, i}$ the concentration in the macropores, $z_{i}$ the valence of the ion and $\Delta \phi_{\mathrm{D}}$ the Donnan potential.

The Boublik-Mansoori-Carnahan-Starling-Leland (BMCSL) equation can be used to calculate $\Delta \mu_{i}^{\text {ex }}$ for an electrolyte with multiple counterions [29, 32, 192]. The BMCSL equation, which we will not discuss in full detail in this work, describes $\Delta \mu_{i}^{\text {ex }}$ as function of the hard-sphere diameter of ion $i$, which is used as a fitting parameter, and a factor that represents the volume fraction occupied by all finite sized ions [32]. Suss showed that, in equilibrium, theory including 
Eq. (8.3) and the BMCSL equation predicts a preferential adsorption of $\mathrm{K}^{+}$over $\mathrm{Na}^{+}$of $1.22-1.3$, and we measured a ratio close to 1.27 .

To conclude, we showed that we can measure ion selective adsorption and that the dynamic porous electrode theory as used in this work describes selective ion adsorption for short CTs well. However, for longer CTs, when we reach equilibrium conditions, the standard theory fails and predicts an equal adsorption of $\mathrm{Na}^{+}$and $\mathrm{K}^{+}$ions, while this is not observed. Suss showed that, extending equilibrium theory with the BMCSL equation will overcome this limitation.

\subsection{Long-term operation and $\mathrm{pH}$ changes}

In Chapter 6 we compared long-term term stability of classical CDI and MCDI cells treating water containing dissolved oxygen. We found that, both in classical CDI and MCDI, the presence of dissolved oxygen in the feed water results in the formation of chemical charged groups at the porous carbon material during operation of the CDI cell. In classical CDI, these groups result in a decrease of desalination performance, whereas in MCDI performance does not decrease. The stable performance of MCDI cells can be explained by the presence of IEMs. Because of the IEMs, co-ions desorbed from EDLs are trapped in the electrodes; they cannot flow from the electrodes into the spacer channel. Therefore, the charge efficiency of MCDI experiments is mainly determined by the selectivity of the membranes, not by the surface chemistry of the electrodes.

A process that can affect the long-term stability of MCDI cells, is that the $\mathrm{pH}$ in the cell can strongly change during operation. High values of $\mathrm{pH}$ can result in precipitation of salt on the membranes, and consequently affect the long-term stability of the system. Therefore, it is important to understand the cause of these changes, and, if possible, change the mode of operation of the cell such that precipitation of salt can be avoided.

In literature, these changes are often attributed to faradaic reactions that occur during operation. However, non-faradaic processes, such as the fact that ions have different diffusion coefficients, and thus different rates of adsorption, can result in $\mathrm{pH}$ changes as well. As we know that these non-faradaic processes occur during operation anyway, and that faradaic reactions may occur, we decided to study the effect of non-faradaic processes at first. To that end, we built a model including ion transport and adsorption of $\mathrm{Na}^{+}, \mathrm{Cl}^{-}, \mathrm{H}^{+}$and $\mathrm{OH}^{-}$ions and we included the water dissociation equilibrium reaction, $\mathrm{H}_{2} \mathrm{O} \rightleftarrows \mathrm{H}^{+}+\mathrm{OH}^{-}$. The model predicted that these non-faradaic processes only explain $\mathrm{pH}$ changes to limited extend, and that we need to study faradaic reactions to explain $\mathrm{pH}$ changes to its full extent.

For future work, it may be interesting to identify which (combination of) 
faradaic reactions result in $\mathrm{pH}$ changes. Theoretically, this identification can be done by incorporating different reactions in a model and compare theoretical results with experimental data. However, exact values of kinetic reaction rate constants are required, but even if these values are known or precisely determined, it will still be difficult to discriminate the contribution of each reaction to $\mathrm{pH}$ changes. Experimentally, we found that it takes a large number of cycles, and thus a long time (order of a few days), to reach dynamic steady state for $\mathrm{pH}$, which is the condition that the $\mathrm{pH}$ signal as function of time of a certain cycle is identical to its previous cycle. This finding implies that the contribution of different faradaic reactions and non-faradaic processes to the observed $\mathrm{pH}$ changes varies over time.

Perhaps, full understanding of the contribution of each faradaic reaction is not required to reduce $\mathrm{pH}$ changes. The model described in Chapter 7, which includes only one faradaic reaction, can be fitted with experimental data, without considering each reaction individually. Then, the model can be used to investigate which operational conditions (CC or CV, both with its own set of operational parameters) minimize the $\mathrm{pH}$ changes. Thereafter, these operational conditions can be experimentally verified. Thus, without the need to obtain more information about the reactions occurring, the model can be used for predictive purposes.

For future modeling work, it will be interesting to couple the amph-D theory with transport theory for fully discretized electrodes. Then, the symmetry assumption of equal salt concentration profiles across the electrodes, an assumption we included in Chapter 7, can be relaxed. Relaxing this assumption will allow for more accurate dynamic modeling of salt adsorption in MCDI, but the theory can then also be applied to classical CDI to model, amongst others, $\mathrm{pH}$ changes. Also, we can extend the amph-D theory to model the binding or dissociation of protons to negatively charged surface groups, such as $\mathrm{COO}^{-}$, or the binding of hydroxyl ions to positively charged groups, such as $\mathrm{H}^{+}$. The degree of ionization of chemical surface charge groups is then dependent on the pK-value of the groups and on the Donnan potential, see Refs. [193, 194]. We can couple this extended amph-D theory to transport theory.

\subsection{Perspectives on technology development}

Having addressed in previous Sections the topics I) energy consumption and resistance identification, II) ion-selective adsorption, and III) long-term operation and $\mathrm{pH}$ changes, we are now in a position to evaluate the suitability of classical CDI and MCDI as methods of desalination.

Firstly, considering energy consumption, Zhao et al. [27] concluded in 2013 that MCDI, in comparison with RO, is a potentially relevant technology for the 
desalination of brackish water, when the salt concentration of the feed water is below $30 \mathrm{mM}(\sim 2 \mathrm{~g} / \mathrm{L})$. However, in their analysis, values for $\Delta c$ and WR are not the same for RO and MCDI. As we discussed in Chapter 4, one can only compare two modes of operation, or more generally, two methods for desalination, in case the desalination objective, which includes the parameters $\Delta c$ and WR, is the same. To ascertain that these parameters have the same value for all methods that are compared, and at the same time that we compare energy consumption of optimized desalination cycles, we must do many experiments (or run many model calculations). This implies that comparing desalination technologies in a fair manner is a challenging task, even when the comparison is only based on energy consumption.

Though energy consumption is important, in reality it is not the only criterion used in the selection of a desalination technology. In such a decision process there are many other criteria used as well which need to be included in a proper socio-economic evaluation, such as:

- desalination performance as described by $\Delta c$ and WR;

- robustness to deal with variations in quantity and quality of the feed water;

- the need for chemicals to clean the membranes;

- potential to apply a technology on a small or large scale;

- environmental impact of a technology including its production, operation and re-use;

- long-term stability, or life-time.

Regarding long-term stability, in Chapter 6 we showed that, for classical CDI without membranes, desalination performance decreases very rapidly over time if dissolved oxygen is present in the feed water. In contrast, for MCDI, we did not observe a decrease in charge efficiency during operation, but we still found that the surface chemistry of the electrode materials changed over time, which is most likely due to oxidation reactions caused by dissolved oxygen. Furthermore, also in MCDI we observed a decrease over time in electrode charge density and capacitance. This decrease will, in long-term experiments, result in an increase of energy consumption, or a reduction in desalination performance. Although we only found evidence for oxidation reactions that lead to charged surface groups, also complete oxidation of carbon material to $\mathrm{CO}_{2}$ is possible. If indeed the carbon itself oxidizes to $\mathrm{CO}_{2}$, performance of MCDI cells will also decrease over time. These problems related to electrode stability limit applicability of MCDI as a desalination technology for water containing dissolved oxygen. In order to improve electrode stability, it is important to find and investigate electrode materials that are stable, have a high salt adsorption capacity and are electronically conductive [195]. Not only electrodes, but also membranes may degrade in time [196]. 
In present Section, we discussed how the content of this Thesis relates to water scarcity problems and the need for desalination technologies. However, the results obtained in this Thesis also have relevance for other topics in science and technology. This is because the principle of CDI has a very general scientific nature, and has broader applicability, also for other technologies that depend on the storage and release of electrical charge by porous carbon electrodes, and the adsorption and release of charged compounds from (aqueous and non-aqueous) electrolytes. The principle of CDI can be applied in various (industrial) separation processes to remove harmful compounds, or to concentrate valuable materials.

As an example of the diversity of potential applications, the principle of charge storage by porous electrodes can also be applied to store electrical energy, for instance with the aim to buffer a temporal mismatch between supply and demand of (renewable) electricity. Devices employing porous electrodes for charge storage are referred to as supercapacitors and are, in terms of design, very similar to CDI cells. Although the application is completely different, the theoretical framework to describe supercapacitors is similar to the framework we use. The results obtained in this Thesis, especially related to the quantification of resistances and energy losses (Chapter 3), and related to the study of mechanisms of ion transport and adsorption (Chapters 2, 3 and 5) including the effect of acid-base and faradaic reactions (Chapter 7), are also of importance for research on energy storage devices and other related technologies that use capacitive electrodes in electrochemical engineering. 
Summary 


\section{Basics of Capacitive Deionization (Chapter 1 \& 2)}

Capacitive deionization (CDI) is an electrochemical technology to adsorb ions from solution by alternately charging and discharging two porous electrodes. During charging, a voltage is applied between the electrodes, and ions are adsorbed into electrical double layers (EDLs) formed in the micropores of the electrode. As a result, feed water is desalinated. After the electrodes are saturated with salt, they are discharged and ions are released, resulting in a concentrated effluent stream. Recently there has been an growing scientific and commercial interest in CDI technology, and various applications are considered, such as wastewater remediation for cooling towers, water softening, and desalination of brackish water. In this Thesis we study mechanisms of ion transport and adsorption in CDI technology, and we address three topics: I) energy consumption and resistance identification, II) ion-selective adsorption, and III) long-term operation and $\mathrm{pH}$ changes.

A key parameter to describe ion adsorption in CDI is charge efficiency, $\Lambda$, which is the ratio of salt adsorption over charge transferred in a desalination cycle. Values for $\Lambda$ in CDI are typically in the range of $0.5-0.8$, significantly less than the theoretical maximum of unity, due to the fact that not only counterions are adsorbed into the pores of the carbon electrodes, but at the same time coions are released. Enhancing the value of $\Lambda$ is advantageous as, for the same amount of salt removed, the total charge transferred decreases, resulting in a lower energy consumption.

To increase $\Lambda$ to values close to unity, ion exchange membranes (IEMs) can be placed in front of the electrodes, which block coions from leaving the electrode region. We refer to this cell design including membranes as Membrane Capacitive Deionization (MCDI), which is a sub-class of CDI.

In this Thesis we investigate a route to increase $\Lambda$ in classical CDI (thus, without membranes), namely by increasing the cell voltage during discharge, compared to the conventional discharge voltage of $0 \mathrm{~V}$. We support this finding by an equilibrium model describing salt adsorption in EDLs. We couple this model to an ion transport model to analyze energy consumption, resistances, and ion-selective adsorption.

\section{Energy consumption and resistance identification (Chapter 3, 4 \& 8)}

In this part of the Thesis, we describe methodology to identify electronic and ionic resistances in (M)CDI. One element of this methodology is that we derive a full-scale (M)CDI model that describes ion transport and adsorption. This model is successfully validated against experimental data and used to calculate the ionic 
resistances across the MCDI cell. We identify that for inflow salt concentrations of $20 \mathrm{mM}$ the resistance is mainly located in the spacer channel and the external electrical circuit, not in the electrodes. Based on these findings, we show that the carbon electrode thickness can be increased without significantly increasing the energy consumption, which has the advantage that the desalination time can be lengthened significantly. Furthermore, we analyze the energy consumption of a desalination cycle in MCDI and we find that only a limited part of the energy invested during desalination is lost because of ionic and electronic resistances. Thus, employing energy recovery technology with an efficiency of $100 \%$, would allow the recovery of $\sim 75 \%$ of the energy that is invested during desalination (of the particular cycle analyzed in this study).

Furthermore, we study the energy consumption of desalination with two modes of operation in CDI: constant current (CC) and constant voltage (CV). Important in the analysis is to precisely define the desalination objective function, such as that per unit total operational time a certain desalination quantity and water recovery must be achieved. We propose that one must analyze the full range of combinations of cycle times, voltages and currents, and only compare the cycles with lowest energy consumption, to come to a rigorous conclusion about which operational mode is optimal for a given desalination objective. We find that without energy recovery for the system we study there is no difference in energy consumption between CC and CV operation. Including 50\% energy recovery, we find for our system that $\mathrm{CC}$ is somewhat more energy efficient but also in $\mathrm{CV}$ much energy can be recovered.

\section{Ion-selective adsorption (Chapter 5 \& 8)}

In this part of the Thesis, we study ion adsorption in CDI from a mixed solution containing two monovalent cations with similar radius, namely $\mathrm{K}^{+}$and $\mathrm{Na}^{+}$. We introduce a novel method to analyze the effluent concentration of a salt solution with multiple ionic species by directing the outflow of a CDI cell to an inductively coupled plasma optical emission spectroscopy (ICP-OES) instrument. We find that $\mathrm{K}^{+}$ions are preferentially adsorbed over $\mathrm{Na}^{+}$ions, due to their higher mobility. Furthermore, we compare our experimental findings with a multicomponent ion transport and adsorption model that calculates dynamic adsorption of ions from solutions of multiple salts. Whereas we find good agreement between data and theory at short cycle times, we observe a considerable discrepancy at higher values. 


\section{Long-term operation and $\mathrm{pH}$ changes (Chapter 6, 7 \& 8)}

In the final part of this Thesis, we compare the desalination performance of CDI and MCDI cells that treats water with and without dissolved oxygen. We show that the desalination performance of CDI (thus, without membranes) decreases over time, most strongly when a cell desalinates water with dissolved oxygen. We describe experimental data with the amphoteric Donnan model and find that an increase with time of negative chemical surface charge in the anode and of positive charge in the cathode explains the decreasing desalination performance. Similar experiments for MCDI (thus, with membranes) show that, although dissolved oxygen affects the surface chemistry of the electrodes, the desalination performance does not decrease over time. We find that, for MCDI, $\Lambda$ is mainly determined by the selectivity of the membranes, and not by the surface chemistry of the electrodes, even though that is affected by dissolved oxygen. Based on this finding, we conclude that, with activated carbon electrodes, from an application point of view, MCDI seems to be the most promising process.

Finally, we study the large differences in $\mathrm{pH}$ that can develop between feed and effluent water of an MCDI cell. These $\mathrm{pH}$ changes can affect the long-term stability of membranes and electrodes. Often, in literature, faradaic reactions are implicated to explain these $\mathrm{pH}$ changes. However, quantitative theory was not yet developed to underpin this explanation. We develop a theory for electrochemical water desalination which includes not only faradaic reactions but also the fact that all ions in the water, including $\mathrm{H}^{+}$and $\mathrm{OH}^{-}$, have different diffusion coefficients. We derive a dynamic model and include the following phenomena: I) different coefficients of various ions, combined with acid-base equilibrium reactions; II) chemical surface charge groups in the micropores of the porous carbon electrodes, where EDLs are formed; and III) faradaic reactions in the micropores. The theory predicts small $\mathrm{pH}$ changes during desalination cycles in MCDI if we only consider phenomena I) and II), but predicts that these $\mathrm{pH}$ changes can be much stronger if we consider phenomenon III) as well, which is in line with earlier statements in the literature on the relevance of faradaic reactions to explain $\mathrm{pH}$ fluctuations. 
Bibliography 
[1] P. M. Biesheuvel, M. Z. Bazant, R. D. Cusick, T. A. Hatton, K. B. Hatzell, M. C. Hatzell, P. Liang, S. Lin, S. Porada, J. G. Santiago, K. C. Smith, M. Stadermann, X. Su, X. Sun, T. D. Waite, A. van der Wal, J. Yoon, R. Zhao, L. Zou, M. Suss, Capacitive Deionization - defining a class of desalination technologies 1-3.arXiv: 1709.05925v1.

[2] M. E. Suss, S. Porada, X. Sun, P. M. Biesheuvel, J. Yoon, V. Presser, Water desalination via capacitive deionization: what is it and what can we expect from it?, Energy \& Environmental Science 8 (2015) 2296-2319. doi:10.1039/C5EE00519A.

[3] P. Srimuk, M. Zeiger, N. Jäckel, A. Tolosa, B. Krüner, S. Fleischmann, I. Grobelsek, M. Aslan, B. Shvartsev, M. E. Suss, V. Presser, Enhanced performance stability of carbon / titania hybrid electrodes during capacitive deionization of oxygen saturated saline water, Electrochimica Acta 224 (2017) 314-328. doi:10.1016/j.electacta.2016.12.060.

[4] Y. Qu, T. F. Baumann, J. G. Santiago, M. Stadermann, Characterization of Resistances of a Capacitive Deionization System, Environmental Science \& Technology 49 (2015) 9699-9706. doi:10.1021/acs.est.5b02542.

[5] G. J. Doornbusch, J. E. Dykstra, P. M. Biesheuvel, M. E. Suss, Fluidized bed electrodes with high carbon loading for water desalination by capacitive deionization, Journal of Materials Chemistry A 4 (2016) 3642-3647. doi:10.1039/C5TA10316A.

[6] W. Tang, P. Kovalsky, D. He, T. D. Waite, Fluoride and nitrate removal from brackish groundwaters by batch-mode capacitive deionization, Water Research 84 (2015) 342-349. doi:10.1016/j.watres.2015.08.012.

[7] Y. Gendel, A. K. E. Rommerskirchen, O. David, M. Wessling, Batch mode and continuous desalination of water using flowing carbon deionization (FCDI) technology, Electrochemistry Communications 46 (2014) 152-156. doi:10.1016/j. elecom.2014.06.004.

[8] P. Nativ, Y. Badash, Y. Gendel, New insights into the mechanism of flow-electrode capacitive deionization, Electrochemistry Communications 76 (2017) 24-28. doi: $10.1016 / \mathrm{j}$. elecom.2017.01.008.

[9] M. C. Hatzell, M. Raju, V. J. Watson, A. G. Stack, A. C. T. van Duin, B. E. Logan, Effect of Strong Acid Functional Groups on Electrode Rise Potential in Capacitive Mixing by Double Layer Expansion, Environmental Science \& Technology 48 (2014) 14041-14048. doi:10.1021/es5043782.

[10] T. Mubita, S. Porada, P. M. Biesheuvel, A. van der Wal, J. E. Dykstra, Capacitive deionization with wire-shaped electrodes, Electrochimica Acta 270 (2018) 165173. doi:10.1016/j.electacta.2018.03.082.

[11] S. Porada, R. Zhao, A. van der Wal, V. Presser, P. M. Biesheuvel, Review on the science and technology of water desalination by capacitive deionization, Progress in Materials Science 58 (2013) 1388-1442. doi:10.1016/j . pmatsci.2013.03.005.

[12] H. Marsh, Activated carbon, 1st Edition, Elsevier, Boston, MA, 2006.

[13] M. M. Mekonnen, A. Y. Hoekstra, Four billion people facing severe water scarcity, Science Advances 2 (2016) e1500323. doi:10.1126/sciadv.1500323.

[14] World Economic Forum, Global Risks 2015: 10th Edition.

[15] S. L. Postel, G. C. Daily, P. R. Ehrlich, Human Appropriation of Renewable Fresh 
Water, Science 271 (1996) 785-788. doi:10.1126/science.271.5250.785.

[16] M. A. Shannon, P. W. Bohn, M. Elimelech, J. G. Georgiadis, B. J. Marinas, A. M. Mayes, Science and technology for water purification in the coming decades, Nature 452 (2008) 301-310. doi:10.1038/nature06599.

[17] G. Amy, N. Ghaffour, Z. Li, L. Francis, R. V. Linares, T. Missimer, S. Lattemann, Membrane-based seawater desalination: Present and future prospects, Desalination 401 (2017) 16-21. doi:10.1016/j.desal.2016.10.002.

[18] L. F. Greenlee, D. F. Lawler, B. D. Freeman, B. Marrot, P. Moulin, Reverse osmosis desalination: Water sources, technology, and today's challenges, Water Research 43 (2009) 2317-2348. doi:10.1016/j.watres.2009.03.010.

[19] A. D. Khawaji, I. K. Kutubkhanah, J.-M. Wie, Advances in seawater desalination technologies, Desalination 221 (2008) 47-69. doi:10.1016/j.desal.2007.01. 067.

[20] Y. Zhou, R. S. J. Tol, Evaluating the costs of desalination and water transport, Water Resources Research 41 (2005) 1-10. doi:10.1029/2004WR003749.

[21] B. Peñate, L. García-Rodríguez, Current trends and future prospects in the design of seawater reverse osmosis desalination technology, Desalination 284 (2012) 1-8. doi:10.1016/j.desal.2011.09.010.

[22] Y. S. Oren, P. M. Biesheuvel, Theory of Ion and Water Transport in ReverseOsmosis Membranes, Physical Review Applied 9 (2018) 024034. doi:10.1103/ PhysRevApplied.9.024034.

[23] M. Busch, W. Mickols, Reducing energy consumption in seawater desalination, Desalination 165 (2004) 299-312. doi:10.1016/j.desal.2004.06.035.

[24] M. Elimelech, W. A. Phillip, The Future of Seawater Desalination: Energy, Technology, and the Environment, Science 333 (2011) 712-717. doi:10.1126/ Science.1200488.

[25] A. Al-Karaghouli, L. L. Kazmerski, Energy consumption and water production cost of conventional and renewable-energy-powered desalination processes, Renewable and Sustainable Energy Reviews 24 (2013) 343-356. doi:10.1016/j. rser.2012.12.064.

[26] S. Lattemann, T. Höpner, Environmental impact and impact assessment of seawater desalination, Desalination 220 (2008) 1-15. doi:10.1016/j.desal.2007. 03.009 .

[27] R. Zhao, S. Porada, P. M. Biesheuvel, A. van der Wal, Energy consumption in membrane capacitive deionization for different water recoveries and flow rates, and comparison with reverse osmosis, Desalination 330 (2013) 35-41. doi:10. 1016/j.desal.2013.08.017.

[28] Y.-J. J. Kim, J.-H. H. Kim, J.-H. H. Choi, Selective removal of nitrate ions by controlling the applied current in membrane capacitive deionization (MCDI), Journal of Membrane Science 429 (2013) 52-57. doi:10.1016/J.Memsci.2012. 11.064 .

[29] B. Giera, N. Henson, E. M. Kober, M. S. Shell, T. M. Squires, Electric DoubleLayer Structure in Primitive Model Electrolytes: Comparing Molecular Dynamics with Local-Density Approximations, Langmuir 31 (2015) 3553-3562. doi:10. 
1021/1a5048936.

[30] C. Prehal, D. Weingarth, E. Perre, R. T. Lechner, H. Amenitsch, O. Paris, V. Presser, Tracking the structural arrangement of ions in carbon supercapacitor nanopores using in situ small-angle X-ray scattering, Energy \& Environmental Science 8 (2015) 1725-1735. doi:10.1039/C5EE00488H.

[31] B. Kastening, M. Heins, Properties of electrolytes in the micropores of activated carbon, Electrochimica Acta 50 (2005) 2487-2498. doi:10.1016/J.Electacta. 2004.10.077.

[32] M. E. Suss, Size-Based Ion Selectivity of Micropore Electric Double Layers in Capacitive Deionization Electrodes, Journal of The Electrochemical Society 164 (2017) 270-275. doi:10.1149/2.1201709jes.

[33] P. M. Biesheuvel, H. V. M. Hamelers, M. E. Suss, Theory of Water Desalination by Porous Electrodes with Immobile Chemical Charge, Colloids and Interface Science Communications 9 (2015) 1-5. doi:10.1016/j.colcom.2015.12.001.

[34] J.-B. B. Lee, K.-K. K. Park, H.-M. M. Eum, C.-W. W. Lee, Desalination of a thermal power plant wastewater by membrane capacitive deionization, Desalination 196 (2006) 125-134. doi:10.1016/j.desal.2006.01.011.

[35] R. Zhao, O. Satpradit, H. H. M. Rijnaarts, P. M. Biesheuvel, A. van der Wal, Optimization of salt adsorption rate in membrane capacitive deionization, Water Research 47 (2013) 1941-1952. doi:10.1016/j.watres.2013.01.025.

[36] J.-H. Choi, Comparison of constant voltage (CV) and constant current (CC) operation in the membrane capacitive deionisation process, Desalination and Water Treatment 56 (2015) 921-928. doi:10.1080/19443994.2014.942379.

[37] Y. J. Kim, J. H. Choi, Improvement of desalination efficiency in capacitive deionization using a carbon electrode coated with an ion-exchange polymer, Water Research 44 (2010) 990-996. doi:10.1016/j.watres.2009.10.017.

[38] H. B. Li, L. Zou, Ion-exchange membrane capacitive deionization: A new strategy for brackish water desalination, Desalination 275 (2011) 62-66. doi:10.1016/j. desal.2011.02.027.

[39] P. M. Biesheuvel, M. Z. Bazant, Nonlinear dynamics of capacitive charging and desalination by porous electrodes, Physical Review E 81 (2010) 031502. doi: 10.1103/Physreve.81.031502.

[40] P. M. Biesheuvel, Y. Fu, M. Z. Bazant, Diffuse charge and Faradaic reactions in porous electrodes, Physical Review E 83 (2011) 061507. doi:10.1103/PhysRevE. 83.061507.

[41] P. M. Biesheuvel, Y. Fu, M. Z. Bazant, Electrochemistry and capacitive charging of porous electrodes in asymmetric multicomponent electrolytes, Russian Journal of Electrochemistry 48 (2012) 580-592. doi:10.1134/S1023193512060031.

[42] S. Porada, L. Borchardt, M. Oschatz, M. Bryjak, J. S. Atchison, K. J. Keesman, S. Kaskel, P. M. Biesheuvel, V. Presser, Direct prediction of the desalination performance of porous carbon electrodes for capacitive deionization, Energy \& Environmental Science 6 (2013) 3700-3712. doi:10.1039/c3ee42209g.

[43] O. Stern, Zur Theorie der Elektrolytischen Doppelschicht, Z. Elektrochem. Angew. Phys. Chem. 30 (1924) 508-516. doi:10.1002/bbpc.192400182. 
[44] K.-L. Yang, T.-Y. Ying, S. Yiacoumi, C. Tsouris, E. S. Vittoratos, Electrosorption of Ions from Aqueous Solutions by Carbon Aerogel: An Electrical Double-Layer Model, Langmuir 17 (2001) 1961-1969. doi:10.1021/la001527s.

[45] T.-Y. Ying, K.-L. Yang, S. Yiacoumi, C. Tsouris, Electrosorption of Ions from Aqueous Solutions by Nanostructured Carbon Aerogel, Journal of Colloid and Interface Science 250 (2002) 18-27. doi:10.1006/jcis.2002.8314.

[46] D. Brogioli, Extracting Renewable Energy from a Salinity Difference Using a Capacitor, Physical Review Letters 103 (2009) 058501. doi:10.1103/PhysRevLett. 103.058501.

[47] R. Zhao, M. van Soestbergen, H. H. M. Rijnaarts, A. van der Wal, M. Z. Bazant, P. M. Biesheuvel, Time-dependent ion selectivity in capacitive charging of porous electrodes, Journal of Colloid and Interface Science 384 (2012) 38-44. doi:Doi10. 1016/J. Jcis.2012.06.022.

[48] R. Zhao, P. M. Biesheuvel, H. Miedema, H. Bruning, A. van der Wal, Charge Efficiency: A Functional Tool to Probe the Double-Layer Structure Inside of Porous Electrodes and Application in the Modeling of Capacitive Deionization, The Journal of Physical Chemistry Letters 1 (2010) 205-210. doi:10.1021/jz900154h.

[49] P. M. Biesheuvel, S. Porada, M. Levi, M. Z. Bazant, Attractive forces in microporous carbon electrodes for capacitive deionization, Journal of Solid State Electrochemistry 18 (2014) 1365-1376. doi:10.1007/s10008-014-2383-5.

[50] H. A. Arafat, M. Franz, N. G. Pinto, Effect of Salt on the Mechanism of Adsorption of Aromatics on Activated Carbon, Langmuir 15 (1999) 5997-6003. doi:10.1021/la9813331.

[51] X. Gao, S. Porada, A. Omosebi, K. Liu, P. M. Biesheuvel, J. Landon, Complementary surface charge for enhanced capacitive deionization, Water Research 92 (2016) 275-282. doi:10.1016/j.watres.2016.01.048.

[52] R. de Levie, On porous electrodes in electrolyte solutions I,II,III, Electrochimica Acta 8 (1963) 751-780. doi:10.1016/0013-4686(63)80042-0.

[53] R. de Levie, On porous electrodes in electrolyte solutions IV, Electrochimica Acta 9 (1964) 1231-1245. doi:10.1016/0013-4686(64)85015-5.

[54] F. A. Posey, T. Morozumi, Theory of potentiostatic and galvanostatic charging of the double layer in porous electrodes, Journal of the Electrochemical Society 113 (1966) 176-184. doi:10.1149/1.2423897.

[55] J. S. Newman, C. W. Tobias, Theoretical Analysis of Current Distribution in Porous Electrodes, Journal of the Electrochemical Society 109 (1962) 1183-1191. doi:10.1149/1.2425269.

[56] A. M. Johnson, J. Newman, Desalting by Means of Porous Carbon Electrodes, Journal of the Electrochemical Society 118 (1971) 510-517. doi:10.1149/1. 2408094.

[57] A. J. Bard, L. R. Faulkner, Electrochemical Methods - Fundamentals and Applications, 2nd Edition, John Wiley \& Sons, Inc., 2010.

[58] F. Duan, X. Du, Y. Li, H. Cao, Y. Zhang, Desalination stability of capacitive deionization using ordered mesoporous carbon : Effect of oxygen-containing surface groups and pore properties, Desalination 376 (2015) 17-24. doi:10.1016/j . 
desal.2015.08.009.

[59] T. Kim, J. Yu, C. Kim, J. Yoon, Hydrogen peroxide generation in flow-mode capacitive deionization, Journal of Electroanalytical Chemistry 776 (2016) 101104. doi:10.1016/j.elechem.2016.07.001.

[60] J.-H. H. Lee, W.-S. S. Bae, J.-H. H. Choi, Electrode reactions and adsorption/desorption performance related to the applied potential in a capacitive deionization process, Desalination 258 (2010) 159-163. doi:10.1016/J.Desal.2010. 03.020.

[61] B. Shapira, E. Avraham, D. Aurbach, Side Reactions in Capacitive Deionization (CDI) Processes: The Role of Oxygen Reduction, Electrochimica Acta 220 (2016) 285-295. doi:10.1016/j.electacta.2016.10.127.

[62] W. Tang, D. He, C. Zhang, P. Kovalsky, T. D. Waite, Comparison of Faradaic reactions in capacitive deionization (CDI) and membrane capacitive deionization (MCDI) water treatment processes, Water Research 120 (2017) 229-237. doi: 10.1016/j.watres.2017.05.009.

[63] X. Gao, A. Omosebi, N. Holubowitch, J. Landon, K. Liu, Capacitive Deionization Using Alternating Polarization: Effect of Surface Charge on Salt Removal, Electrochimica Acta 233 (2017) 249-255. doi:10.1016/j.electacta.2017.03.021.

[64] I. Cohen, E. Avraham, Y. Bouhadana, A. Soffer, D. Aurbach, The effect of the flow-regime, reversal of polarization, and oxygen on the long term stability in capacitive de-ionization processes, Electrochimica Acta 153 (2015) 106-114. doi: 10.1016/j.electacta.2014.12.007.

[65] N. Holubowitch, A. Omosebi, X. Gao, J. Landon, K. Liu, Quasi-steady state polarization reveals the interplay of capacitive and faradaic processes in capacitive deionization, ChemElectroChem 4 (2017) 2404-2413. doi:10.1002/celc. 201700082.

[66] R. De Levie, A pH centenary, Electrochimica Acta 135 (2014) 604-639. doi: 10.1016/j.electacta.2014.04.006.

[67] J. Liu, M. Lu, J. Yang, J. Cheng, W. Cai, Capacitive desalination of $\mathrm{ZnO} /$ activated carbon asymmetric capacitor and mechanism analysis, Electrochimica Acta 151 (2015) 312-318. doi:10.1016/j.electacta.2014.11.023.

[68] O. Nir, N. F. Bishop, O. Lahav, V. Freger, Modeling pH variation in reverse osmosis, Water Research 87 (2015) 328-335. doi:10.1016/j.watres.2015.09. 038.

[69] A. Frumkin, Wasserstoffuberspannung und Struktur der Doppelschicht, Z. Physik. Chem. 164A (1933) 121.

[70] M. van Soestbergen, Frumkin-Butler-Volmer theory and mass transfer in electrochemical cells, Russian Journal of Electrochemistry 48 (2012) 570-579. doi: 10.1134/S1023193512060110.

[71] R. Zhao, P. M. Biesheuvel, A. van der Wal, Energy consumption and constant current operation in membrane capacitive deionization, Energy \& Environmental Science 5 (2012) 9520-9527. doi:10.1039/C2ee21737f.

[72] S. I. Jeon, H. R. Park, J. G. Yeo, S. Yang, C. H. Cho, M. H. Han, D. K. Kim, Desalination via a new membrane capacitive deionization process utilizing flow- 
electrodes, Energy \& Environmental Science 6 (2013) 1471-1475. doi:10.1039/ C3ee24443a.

[73] S. Jeon, J. Yeo, S. Yang, J. Choi, D. K. Kim, Ion storage and energy recovery of a flow-electrode capacitive deionization process, Journal of Materials Chemistry A 2 (2014) 6378. doi:10.1039/c4ta00377b.

[74] K. B. Hatzell, E. Iwama, A. Ferris, B. Daffos, K. Urita, T. Tzedakis, F. Chauvet, P. L. Taberna, Y. Gogotsi, P. Simon, Capacitive deionization concept based on suspension electrodes without ion exchange membranes, Electrochemistry Communications 43 (2014) 18-21. doi:10.1016/j.elecom.2014.03.003.

[75] S. Yang, J. Choi, J.-G. Yeo, S.-i. Jeon, H.-r. Park, D. K. Kim, Flow-electrode capacitive deionization using an aqueous electrolyte with a high salt concentration, Environmental Science \& Technology 50 (2016) 5892-5899. doi:10.1021/acs. est. 5 b04640.

[76] S. Porada, D. Weingarth, H. V. M. Hamelers, M. Bryjak, V. Presser, P. M. Biesheuvel, Carbon flow electrodes for continuous operation of capacitive deionization and capacitive mixing energy generation, Journal of Materials Chemistry A 2 (2014) 9313-9321. doi:10.1039/c4ta01783h.

[77] K. B. Hatzell, M. C. Hatzell, K. M. Cook, M. Boota, G. M. Housel, A. Mcbride, E. C. Kumbur, Y. Gogotsi, Effect of Oxidation of Carbon Material on Suspension Electrodes for Flow Electrode Capacitive Deionization, Environmental Science \& Technology 49 (2015) 3040-3047. doi:10.1021/es5055989.

[78] D. He, J. Ma, D. He, W. Tang, P. Kovalsky, C. He, C. Zhang, T. D. Waite, Development of Redox-Active Flow Electrodes for High-Performance Capacitive Deionization, Environmental Science \& Technology 50 (2016) 13495-13501. doi: 10.1021/acs.est. 6b03424.

[79] A. Rommerskirchen, Y. Gendel, M. Wessling, Single module flow-electrode capacitive deionization for continuous water desalination, Electrochemistry Communications 60 (2015) 34-37. doi:10.1016/j.elecom.2015.07.018.

[80] S. Porada, B. B. Sales, H. V. M. Hamelers, P. M. Biesheuvel, Water Desalination with Wires, Journal of Physical Chemistry Letters 3 (2012) 1613-1618. doi: 10.1021/Jz3005514.

[81] B. B. Sales, O. S. Burheim, F. Liu, O. Schaetzle, C. J. N. Buisman, H. V. M. Hamelers, Impact of Wire Geometry in Energy Extraction from Salinity Differences Using Capacitive Technology, Environmental Science \& Technology 46 (2012) 12203-12208. doi:10.1021/es302169c.

[82] J. Rouquerol, D. Avnir, C. W. Fairbridge, D. H. Everett, J. M. e. a. Haynes, Recommendations for the characterization of porous solids (technical report), Tech. rep. (1994).

[83] K. S. W. Sing, D. H. Everett, R. A. V. Haul, L. Moscou, R. A. Pierotti, J. Rouquerol, T. Siemieniewska, Reporting physisorption data for gas/solid systems with special reference to the determination of surface area and porosity (Recommendations 1984), Pure and Applied Chemistry 57 (1985) 603-619. doi:10.1515/iupac.57.0007.

[84] A. Soffer, M. Folman, The electrical double layer of high surface porous carbon 
electrode, J. Electroanal. Chem. 38 (1972) 25-43. doi:10.1016/S0022-0728(72) 80087-1.

[85] J. W. Blair, G. W. Murphy, Electrochemical Demineralization of Water with Porous Electrodes of Large Surface Area, in: Saline Water Conversion, Vol. 27, American Chemical Society, 1960, Ch. 20, pp. 206-223. doi:10.1021/ ba-1960-0027.ch020.

[86] B. B. Arnold, G. W. Murphy, Studies on the electrochemistry of carbon and chemically-modified carbon surfaces, Journal Physical Chemistry 65 (1961) 135138. doi:10.1021/j100819a038.

[87] G. W. Murphy, D. D. Caudle, Mathematical theory of electrochemical demineralization in flowing systems, Electrochimica Acta 12 (1967) 1655-1664. doi: 10.1016/0013-4686(67)80079-3.

[88] I. Cohen, E. Avraham, M. Noked, A. Soffer, D. Aurbach, Enhanced Charge Efficiency in Capacitive Deionization Achieved by Surface-Treated Electrodes and by Means of a Third Electrode, Journal of Physical Chemistry C 115 (2011) 19856-19863. doi:10.1021/Jp206956a.

[89] X. Gao, A. Omosebi, J. Landon, K. Liu, Surface charge enhanced carbon electrodes for stable and efficient capacitive deionization using inverted adsorptiondesorption behavior, Energy \& Environmental Science 8 (2015) 897-909. doi:10.1039/C4EE03172E.

[90] T. Wu, G. Wang, F. Zhan, Q. Dong, Q. Ren, J. Wang, J. Qiu, Surface-treated carbon electrodes with modified potential of zero charge for capacitive deionization, Water Research 93 (2016) 30-37. doi:10.1016/j.watres.2016.02.004.

[91] J. Lee, S. Kim, C. Kim, J. Yoon, Hybrid capacitive deionization to enhance the desalination performance of capacitive techniques, Energy \& Environmental Science 7 (2014) 3683-3689. doi:10.1039/C4EE02378A.

[92] P. Srimuk, L. Ries, M. Zeiger, S. Fleischmann, N. Jäckel, A. Tolosa, B. Krüner, M. Aslan, V. Presser, High performance stability of titania decorated carbon for desalination with capacitive deionization in oxygenated water, RSC Advances 6 (2016) 106081-106089. doi:10.1039/C6RA22800C.

[93] K. C. Smith, R. Dmello, Na-Ion Desalination (NID) Enabled by Na-Blocking Membranes and Symmetric Na-Intercalation: Porous-Electrode Modeling, Journal of The Electrochemical Society 163 (2016) A530-A539. doi:10.1149/2. 0761603jes.

[94] Y. Liu, W. Ma, Z. Cheng, J. Xu, R. Wang, X. Gang, Preparing CNTs/CaSelective zeolite composite electrode to remove calcium ions by capacitive deionization, Desalination 326 (2013) 109-114. doi:10.1016/j.desal.2013.07.022.

[95] Y.-J. J. Kim, J.-H. H. Choi, Selective removal of nitrate ion using a novel composite carbon electrode in capacitive deionization, Water Research 46 (2012) 60336039. doi:10.1016/j.watres.2012.08.031.

[96] C.-H. Hou, C.-Y. Huang, A comparative study of electrosorption selectivity of ions by activated carbon electrodes in capacitive deionization, Desalination 314 (2013) 124-129. doi:10.1016/j.desal.2012.12.029.

[97] Y. Qu, P. G. Campbell, L. Gu, J. M. Knipe, E. Dzenitis, J. G. Santiago, 
M. Stadermann, Energy consumption analysis of constant voltage and constant current operations in capacitive deionization, Desalination 400 (2016) 18-24. doi:10.1016/j.desal.2016.09.014.

[98] A. Hemmatifar, J. W. Palko, M. Stadermann, J. G. Santiago, Energy breakdown in capacitive deionization, Water Research 104 (2016) 303-311. doi:10.1016/j. watres.2016.08.020.

[99] Y. Bouhadana, M. Ben-Tzion, A. Soffer, D. Aurbach, A control system for operating and investigating reactors: The demonstration of parasitic reactions in the water desalination by capacitive de-ionization, Desalination 268 (2011) 253-261. doi:10.1016/j.desal.2010.10.037.

[100] Y. Bouhadana, E. Avraham, M. Noked, M. Ben-Tzion, A. Soffer, D. Aurbach, Capacitive deionization of $\mathrm{NaCl}$ solutions at non-steady-state conditions: Inversion functionality of the carbon electrodes, Journal of Physical Chemistry C 115 (2011) 16567-16573. doi:10.1021/jp2047486.

[101] S. Shanbhag, J. F. Whitacre, M. S. Mauter, The Origins of Low Efficiency in Electrochemical De-Ionization Systems, Journal of The Electrochemical Society 163 (2016) E363-E371. doi:10.1149/2.0181614jes.

[102] B. van Limpt, A. van der Wal, Water and chemical savings in cooling towers by using membrane capacitive deionization, Desalination 342 (2014) 148-155. doi:10.1016/j.desal.2013.12.022.

[103] S. J. Seo, H. Jeon, J. K. Lee, G. Y. Kim, D. Park, H. Nojima, J. Lee, S. H. Moon, Investigation on removal of hardness ions by capacitive deionization (CDI) for water softening applications, Water Research 44 (2010) 2267-2275. doi:10. 1016/J.Watres. 2009.10.020.

[104] H. H. Jung, S. W. Hwang, S. H. Hyun, K. H. Lee, G. T. Kim, Capacitive deionization characteristics of nanostructured carbon aerogel electrodes synthesized via ambient drying, Desalination 216 (2007) 377-385. doi:10.1016/j.desal.2006. 11.023.

[105] R. A. Rica, R. Ziano, D. Salerno, F. Mantegazza, D. Brogioli, Thermodynamic Relation between Voltage-Concentration Dependence and Salt Adsorption in Electrochemical Cells, Physical Review Letters 109 (2012) 156103. doi: 10.1103/PhysRevLett.109.156103.

[106] Z. H. Huang, M. Wang, L. Wang, F. Y. Kang, Relation between the Charge Efficiency of Activated Carbon Fiber and Its Desalination Performance, Langmuir 28 (2012) 5079-5084. doi:10.1021/la204690s.

[107] H. B. Li, C. Y. Nie, L. K. Pan, Z. Sun, The study of membrane capacitive deionization from charge efficiency, Desalination and Water Treatment 42 (2012) 210-215. doi:10.5004/dwt.2012.2824.

[108] G. Wang, B. Qian, Q. Dong, J. Yang, Z. Zhao, J. Qiu, Highly mesoporous activated carbon electrode for capacitive deionization, Separation and Purification Technology 103 (2012) 216-221. doi:10.1016/j.seppur.2012.10.041.

[109] X. Gao, A. Omosebi, J. Landon, K. Liu, Enhancement of charge efficiency for a capacitive deionization cell using carbon xerogel with modified potential of zero charge, Electrochemistry Communications 39 (2014) 22-25. doi:10.1016/ 
j.elecom.2013.12.004.

[110] T. Kim, J. Yoon, Relationship between capacitance of activated carbon composite electrodes measured at a low electrolyte concentration and their desalination performance in capacitive deionization, Journal of Electroanalytical Chemistry 704 (2013) 169-174. doi:10.1016/j.jelechem.2013.07.003.

[111] Y. A. C. Jande, W. S. Kim, Predicting the lowest effluent concentration in capacitive deionization, Separation and Purification Technology 115 (2013) 224-230. doi:10.1016/j.seppur.2013.05.022.

[112] M. E. Suss, T. F. Baumann, W. L. Bourcier, C. M. Spadaccini, K. A. Rose, J. G. Santiago, M. Stadermann, Capacitive desalination with flow-through electrodes, Energy \& Environmental Science 5 (2012) 9511-9519. doi:10.1039/C2ee21498a.

[113] J. Liu, S. Wang, J. Yang, J. Liao, M. Lu, H. Pan, L. An, ZnCl2 activated electrospun carbon nanofiber for capacitive desalination, Desalination 344 (2014) 446453. doi:10.1016/j.desal.2014.04.015.

[114] K. Laxman, M. T. Z. Myint, H. Bourdoucen, J. Dutta, Enhancement in Ion Adsorption Rate and Desalination Efficiency in a Capacitive Deionization Cell through Improved Electric Field Distribution Using Electrodes Composed of Activated Carbon Cloth Coated with Zinc Oxide Nanorods, ACS Applied Materials \& Interfaces 6 (2014) 10113-10120. doi:10.1021/am501041t.

[115] M. Zafra, P. Lavela, G. Rasines, C. Macías, J. Tirado, C. Ania, A novel method for metal oxide deposition on carbon aerogels with potential application in capacitive deionization of saline water, Electrochimica Acta 135 (2014) 208-216. doi:10. 1016/j.electacta.2014.04.182.

[116] L. Han, K. G. Karthikeyan, M. A. Anderson, K. B. Gregory, J. J. Wouters, A. Abdel-Wahab, K. B. Gregory, Mechanistic insights into the use of oxide nanoparticles coated asymmetric electrodes for capacitive deionization, Electrochimica Acta 90 (2013) 573-581. doi:10.1016/j.electacta.2012.11.069.

[117] J.-H. Yeo, J.-H. Choi, Enhancement of nitrate removal from a solution of mixed nitrate, chloride and sulfate ions using a nitrate-selective carbon electrode, Desalination 320 (2013) 10-16. doi:10.1016/j.desal.2013.04.013.

[118] B. M. Asquith, J. Meier-Haack, B. P. Ladewig, Cation exchange copolymer enhanced electrosorption, Desalination 345 (2014) 94-100. doi:10.1016/j.desal. 2014.04 .027$.

[119] Y. Liu, L. Pan, X. Xu, T. Lu, Z. Sun, D. H. Chua, Enhanced desalination efficiency in modified membrane capacitive deionization by introducing ion-exchange polymers in carbon nanotubes electrodes, Electrochimica Acta 130 (2014) 619624. doi:10.1016/j.electacta.2014.03.086.

[120] A. Omosebi, X. Gao, J. Landon, K. Liu, Asymmetric Electrode Configuration for Enhanced Membrane Capacitive Deionization, ACS Applied Materials \& Interfaces 6 (2014) 12640-9. doi:10.1021/am5026209.

[121] T. Kim, H. D. Yoo, S. M. Oh, J. Yoon, Potential Sweep Method to Evaluate Rate Capability in Capacitive Deionization, Electrochimica Acta 139 (2014) 374-380. doi:10.1016/j.electacta.2014.07.034.

[122] M. Mossad, L. Zou, Evaluation of the salt removal efficiency of capacitive deion- 
isation: Kinetics, isotherms and thermodynamics, Chemical Engineering Journal 223 (2013) 704-713. doi:10.1016/j.cej.2013.03.058.

[123] L. Han, K. G. Karthikeyan, M. A. Anderson, K. B. Gregory, Exploring the impact of pore size distribution on the performance of carbon electrodes for capacitive deionization, Journal of Colloid and Interface Science 430 (2014) 93-99. doi: $10.1016 / j \cdot j$ cis.2014.05.015.

[124] H. Li, F. Zaviska, S. Liang, J. Li, L. He, H. Y. Yang, A high charge efficiency electrode by self-assembling sulphonated reduced graphene oxide onto carbon fibre: towards enhanced capacitive deionization, Journal of Materials Chemistry A 2 (2014) 3484. doi:10.1039/c3ta14322h.

[125] E. Avraham, M. Noked, Y. Bouhadana, A. Soffer, D. Aurbach, Limitations of Charge Efficiency in Capacitive Deionization II. On the Behavior of CDI Cells Comprising Two Activated Carbon Electrodes, Journal of the Electrochemical Society 156 (2009) 157-162. doi:10.1149/1.3193709.

[126] I. Cohen, E. Avraham, A. Soffer, D. Aurbach, Water Desalination by Capacitive Deionization - Advantages Limitations and Modification, ECS Transactions 45 (2013) 43-59. doi:10.1149/04517.0043ecst.

[127] Y. A. C. Jande, W. S. Kim, Desalination using capacitive deionization at constant current, Desalination 329 (2013) 29-34. doi:10.1016/j.desal.2013.08.023.

[128] S. Porada, M. Bryjak, A. van der Wal, P. M. Biesheuvel, Effect of electrode thickness variation on operation of capacitive deionization, Electrochimica Acta 75 (2012) 148-156. doi:10.1016/J.Electacta.2012.04.083.

[129] S. Porada, L. Weinstein, R. Dash, A. van der Wal, M. Bryjak, Y. Gogotsi, P. M. Biesheuvel, Water Desalination Using Capacitive Deionization with Microporous Carbon Electrodes, ACS Applied Materials \& Interfaces 4 (2012) 1194-1199. doi: 10.1021/Am201683j.

[130] P. M. Biesheuvel, R. Zhao, S. Porada, A. van der Wal, Theory of membrane capacitive deionization including the effect of the electrode pore space, Journal of Colloid and Interface Science 360 (2011) 239-248. doi:10.1016/J . Jcis.2011. 04.049 .

[131] P. M. Biesheuvel, Y. Q. Fu, M. Z. Bazant, Diffuse charge and Faradaic reactions in porous electrodes, Physical Review E: Statistical, Nonlinear, and Soft Matter Physics E83 (2011) 061507. doi:10.1103/PhysRevE.83.061507.

[132] K. Sharma, R. T. Mayes, J. O. Kiggans Jr, S. Yiacoumi, J. Gabitto, D. W. DePaoli, S. Dai, C. Tsouris, Influence of temperature on the electrosorption of ions from aqueous solutions using mesoporous carbon materials, Separation and Purification Technology 116 (2013) 206-213. doi:10.1016/j.seppur.2013.05. 038.

[133] M. Yaniv, A. Soffer, The Transient Behavior of an Ideally Polarized Porous Carbon Electrode at Constant Charging Current, Journal of the Electrochemical Society 123 (1976) 506-511. doi:10.1149/1.2132864.

[134] V. V. Nikonenko, A. V. Kovalenko, M. K. Urtenov, N. D. Pismenskaya, J. Han, P. Sistat, G. Pourcelly, Desalination at overlimiting currents: State-of-the-art and perspectives, Desalination 342 (2014) 85-106. doi:10.1016/j.desal.2014.01. 
008.

[135] M. Alkuran, M. Orabi, N. Scheinberg, Highly efficient Capacitive De-Ionization (CDI) water purification system using a buck-boost converter, in: Applied Power Electronics Conference and Exposition - Twenty-Third Annual IEEE, 2008, pp. 1926-1930. doi:10.1109/APEC.2008.4522991.

[136] P. Wu, J. Huang, V. Meunier, B. G. Sumpter, R. Qiao, Voltage Dependent Charge Storage Modes and Capacity in Subnanometer Pores, The Journal of Physical Chemistry Letters 3 (2012) 1732-1737. doi:10.1021/jz300506j.

[137] C. Tsouris, R. Mayes, J. Kiggans, K. Sharma, S. Yiacoumi, D. DePaoli, S. Dai, Mesoporous Carbon for Capacitive Deionization of Saline Water, Environmental Science \& Technology 45 (2011) 10243-10249. doi:10.1021/es201551e.

[138] E. Avraham, Y. Bouhadana, A. Soffer, D. Aurbach, Limitation of Charge Efficiency in Capacitive Deionization, Journal of the Electrochemical Society 156 (2009) 95-99. doi:10.1149/1.3115463.

[139] H. Li, L. Pan, Y. Zhang, L. Zou, C. Sun, Y. Zhan, Z. Sun, Kinetics and thermodynamics study for electrosorption of $\mathrm{NaCl}$ onto carbon nanotubes and carbon nanofibers electrodes, Chemical Physics Letters 485 (2010) 161-166. doi:10.1016/j.cplett.2009.12.031.

[140] P. Długołęcki, A. van der Wal, Energy Recovery in Membrane Capacitive Deionization, Environmental Science \& Technology 47 (2013) 4904-4910. doi : 10 . 1021/ es3053202.

[141] O. N. Demirer, R. M. Naylor, C. A. Rios Perez, E. Wilkes, C. Hidrovo, Energetic performance optimization of a capacitive deionization system operating with transient cycles and brackish water, Desalination 314 (2013) 130-138. doi:10.1016/j.desal.2013.01.014.

[142] M. Mirzadeh, F. Gibou, T. M. Squires, Enhanced Charging Kinetics of Porous Electrodes: Surface Conduction as a Short-Circuit Mechanism, Physical Review Letters 113 (2014) 097701. doi:10.1103/PhysRevLett.113.097701.

[143] J. E. Dykstra, P. M. Biesheuvel, H. Bruning, A. Ter Heijne, Theory of ion transport with fast acid-base equilibrations in bioelectrochemical systems, Physical Review E 90 (2014) 013302. doi:10.1103/PhysRevE.90.013302.

[144] A. H. Galama, J. W. Post, M. A. Cohen Stuart, P. M. Biesheuvel, Validity of the Boltzmann equation to describe Donnan equilibrium at the membranesolution interface, Journal of Membrane Science 442 (2013) 131-139. doi:10.1016/j. memsci.2013.04.022.

[145] J. M. Paz-Garcia, J. E. Dykstra, P. M. Biesheuvel, H. V. M. Hamelers, Energy from $\mathrm{CO}_{2}$ using capacitive electrodes A model for energy extraction cycles, Journal of Colloid and Interface Science 442 (2015) 103-109. doi:10.1016/j.jcis. 2014.11 .045$.

[146] M. B. Andersen, M. van Soestbergen, A. Mani, H. Bruus, P. M. Biesheuvel, M. Z. Bazant, Current-induced membrane discharge, Physical Review Letters 109 (2012) 108301. doi:10.1103/Physrevlett.109.108301.

[147] L. X. Tuan, M. Verbanck, C. Buess-Herman, H. Hurwitz, Properties of CMV cation exchange membranes in sulfuric acid media, Journal of Membrane Science 
284 (2006) 67-78. doi:10.1016/j.memsci.2006.06.036.

[148] P. Długołęcki, P. Ogonowski, S. J. Metz, M. Saakes, K. Nijmeijer, M. Wessling, On the resistances of membrane, diffusion boundary layer and double layer in ion exchange membrane transport, Journal of Membrane Science 349 (2010) 369-379. doi:10.1016/j.memsci.2009.11.069.

[149] P. Liang, L. Yuan, X. Yang, S. Zhou, X. Huang, Coupling ion-exchangers with inexpensive activated carbon fiber electrodes to enhance the performance of capacitive deionization cells for domestic wastewater desalination, Water Research 47 (2013) 2523-2530. doi:10.1016/j .watres.2013.02.037.

[150] D. A. Vermaas, M. Saakes, K. Nijmeijer, Power generation using profiled membranes in reverse electrodialysis, Journal of Membrane Science 385386 (2011) 234-242. doi:10.1016/j.memsci.2011.09.043.

[151] X. Su, T. A. Hatton, Redox-electrodes for selective electrochemical separations, Advances in Colloid and Interface Science 244 (2017) 6-20. doi:10.1016/j.cis . 2016.09 .001$.

[152] S. Shanbhag, Y. Bootwala, J. F. Whitacre, M. S. Mauter, Ion Transport and Competition Effects on $\mathrm{NaTi}_{2}\left(\mathrm{PO}_{4}\right)_{3}$ and $\mathrm{Na}_{4} \mathrm{Mn}_{9} \mathrm{O}_{18}$ Selective Insertion Electrode Performance, Langmuir 33 (2017) 12580-12591. doi:10.1021/acs.langmuir. $7 \mathrm{~b} 02861$.

[153] J. Kang, T. Kim, K. Jo, J. Yoon, Comparison of salt adsorption capacity and energy consumption between constant current and constant voltage operation in capacitive deionization, Desalination 352 (2014) 52-57. doi:10.1016/j.desal. 2014.08 .009$.

[154] L. Han, K. G. Karthikeyan, K. B. Gregory, Energy Consumption and Recovery in Capacitive Deionization Using Nanoporous Activated Carbon Electrodes, Journal of The Electrochemical Society 162 (2015) E282-E288. doi: 10.1149/2.0431512jes.

[155] M. Andelman, Flow Through Capacitor basics, Separation and Purification Technology 80 (2011) 262-269. doi:10.1016/j.seppur.2011.05.004.

[156] J. Kang, T. Kim, H. Shin, J. Lee, J.-I. Ha, J. Yoon, Direct energy recovery system for membrane capacitive deionization, Desalination 398 (2016) 144-150. doi:10.1016/j.desal.2016.07.025.

[157] T. Kim, J. E. Dykstra, S. Porada, A. van der Wal, J. Yoon, P. M. Biesheuvel, Enhanced charge efficiency and reduced energy use in capacitive deionization by increasing the discharge voltage, Journal of Colloid and Interface Science 446 (2015) 317-326. doi:10.1016/j.jcis.2014.08.041.

[158] J. E. Dykstra, J. Dijkstra, A. Van der Wal, H. V. M. Hamelers, S. Porada, On-line method to study dynamics of ion adsorption from mixtures of salts in capacitive deionization, Desalination 390 (2016) 47-52. doi:10.1016/j.desal.2016.04. 001.

[159] J. E. Dykstra, K. J. Keesman, P. M. Biesheuvel, A. van der Wal, Theory of $\mathrm{pH}$ changes in water desalination by capacitive deionization, Water Research 119 (2017) 178-186. doi:10.1016/j.watres.2017.04.039.

[160] A. Hemmatifar, M. Stadermann, J. G. Santiago, Two-Dimensional Porous Elec- 
trode Model for Capacitive Deionization, The Journal of Physical Chemistry C 119 (2015) 24681-24694. doi:10.1021/acs.jpcc.5b05847.

[161] P. M. Biesheuvel, Activated carbon is an electron-conducting amphoteric ion adsorbent (2015) 1-9 arXiv:1509.06354.

[162] J. E. Dykstra, R. Zhao, P. M. Biesheuvel, A. van der Wal, Resistance identification and rational process design in Capacitive Deionization, Water Research 88 (2016) 358-370. doi:10.1016/j.watres.2015.10.006.

[163] E. Avraham, M. Noked, A. Soffer, D. Aurbach, The feasibility of boron removal from water by capacitive deionization, Electrochimica Acta 56 (2011) 6312-6317. doi:10.1016/j.electacta.2011.05.037.

[164] J. J. Lado, R. E. Pérez-Roa, J. J. Wouters, M. Isabel Tejedor-Tejedor, M. a. Anderson, Evaluation of operational parameters for a capacitive deionization reactor employing asymmetric electrodes, Separation and Purification Technology 133 (2014) 236-245. doi:10.1016/j.seppur.2014.07.004.

[165] K. C. Leonard, J. R. Genthe, J. L. Sanfilippo, W. A. Zeltner, M. A. Anderson, Synthesis and characterization of asymmetric electrochemical capacitive deionization materials using nanoporous silicon dioxide and magnesium doped aluminum oxide, Electrochimica Acta 54 (2009) 5286-5291. doi:10.1016/j.electacta. 2009.01.082.

[166] T. Ryu, J. C. Ryu, J. Shin, D. H. Lee, Y. H. Kim, K.-S. Chung, Recovery of Lithium by an Electrostatic Field-Assisted Desorption Process, Industrial \& Engineering Chemistry Research 52 (2013) 13738-13742. doi:10.1021/ie401977s.

[167] E. R. Nightingale, Phenomenological Theory of Ion Solvation. Effective Radii of Hydrated Ions, The Journal of Physical Chemistry 63 (1959) 1381-1387. doi: 10.1021/j150579a011.

[168] Y. Bian, X. Yang, P. Liang, Y. Jiang, C. Zhang, X. Huang, Enhanced desalination performance of membrane capacitive deionization cells by packing the flow chamber with granular activated carbon, Water Research 85 (2015) 371-376. doi:10.1016/j.watres.2015.08.058.

[169] M. Aslan, M. Zeiger, N. Jäckel, I. Grobelsek, D. Weingarth, V. Presser, Improved capacitive deionization performance of mixed hydrophobic/hydrophilic activated carbon electrodes, Journal of Physics: Condensed Matter 28 (2016) 114003. doi: 10.1088/0953-8984/28/11/114003.

[170] I. Cohen, E. Avraham, Y. Bouhadana, A. Soffer, D. Aurbach, Long term stability of capacitive de-ionization processes for water desalination: The challenge of positive electrodes corrosion, Electrochimica Acta 106 (2013) 91-100. doi:10.1016/j.electacta.2013.05.029.

[171] A. Omosebi, X. Gao, N. Holubowitch, Z. Li, J. Landon, K. Liu, Anion Exchange Membrane Capacitive Deionization Cells, Journal of The Electrochemical Society 164 (2017) 242-247. doi:10.1149/2.0461709jes.

[172] E. Bayram, E. Ayranci, A systematic study on the changes in properties of an activated carbon cloth upon polarization, Electrochimica Acta 56 (2011) 21842189. doi:10.1016/j.electacta.2010.12.018.

[173] Z. Chen, H. Zhang, C. Yang, X. Sun, H. Guo, Effects of ageing and incorporation 
of ion- exchange membrane on the electrosorption performance of activated carbon based electrodes modules, Desalination and Water Treatment 3994. doi:10.1080/ 19443994.2012.749373.

[174] C. C. Hu, C. C. Wang, Effects of electrolytes and electrochemical pretreatments on the capacitive characteristics of activated carbon fabrics for supercapacitors, Journal of Power Sources 125 (2004) 299-308. doi:10.1016/j.jpowsour. 2003. 08.002 .

[175] D. He, C. E. Wong, W. Tang, P. Kovalsky, T. D. Waite, Faradaic Reactions in Water Desalination by Batch-Mode Capacitive Deionization, Environmental Science \& Technology Letters 3 (2016) 222-226. doi:10.1021/acs.estlett.6b00124.

[176] W. Shen, Z. Li, Y. Liu, Surface Chemical Functional Groups Modification of Porous Carbon, Recent Patents on Chemical Engineering 1 (2008) 27-40. doi: $10.2174 / 2211334710801010027$.

[177] S. S. Barton, M. J. B. Evans, E. Halliop, J. A. F. MacDonald, Acidic and basic sites on the surface of porous carbon, Carbon 35 (1997) 1361-1366. doi:10.1016/ S0008-6223(97)00080-8.

[178] Z. Ogumi, Z. Takehara, S. Yoshizawa, Gas Permeation in SPE Method - 1. oxygen permeation through Nafion and Neosepta, Journal of the Electrochemical Society 131 (1984) 769-773. doi:10.1149/1.2115696.

[179] J. R. Kim, S. Cheng, S. E. Oh, B. E. Logan, Power generation using different cation, anion, and ultrafiltration membranes in microbial fuel cells, Environmental Science \& Technology 41 (2007) 1004-1009. doi:10.1021/es062202m.

[180] K. J. Chae, M. Choi, F. F. Ajayi, W. Park, I. S. Chang, I. S. Kim, Mass Transport through a Proton Exchange Membrane (Nafion) in Microbial Fuel Cells, Energy \& Fuels 22 (2008) 169-176. doi:10.1021/ef700308u.

[181] Y.-J. Kim, J.-H. Choi, Improvement of desalination efficiency in capacitive deionization using a carbon electrode coated with an ion-exchange polymer, Water Research 44 (2010) 990-996. doi:10.1016/j.watres.2009.10.017.

[182] R. A. Rica, R. Ziano, D. Salerno, F. Mantegazza, M. Z. Bazant, D. Brogioli, Electro-diffusion of ions in porous electrodes for capacitive extraction of renewable energy from salinity differences, Electrochimica Acta 92 (2013) 304-314. doi: 10.1016/j.electacta.2013.01.063.

[183] M. Montes-Morán, D. Suárez, J. Menéndez, E. Fuente, On the nature of basic sites on carbon surfaces: an overview, Carbon 42 (2004) 1219-1225. doi:10. 1016/j. carbon. 2004.01.023.

[184] C. A. Leon y Leon, J. M. Solar, V. Calemma, L. R. Radovic, Evidence for the protonation of basal plane sites on carbon, Carbon 30 (1992) 797-811. doi: 10.1016/0008-6223(92)90164-R.

[185] R. J. Helder, Diffusion of inorganic carbon across an unstirred layer: a simplified quantitative approach, Plant, Cell \& Environment 8 (1985) 399-408. doi:10. 1111/j.1365-3040.1985.tb01675.x.

[186] Y. L. Hwang, F. G. Helfferich, Generalized-Model for Multispecies Ion-Exchange Kinetics Including Fast Reversible-Reactions, Reactive Polymers 5 (1987) 237253. doi:10 .1016/0167-6989(87)90233-9. 
[187] C. P. Nielsen, H. Bruus, Transport-limited water splitting at ion-selective interfaces during concentration polarization, Physical Review E 89 (2014) 052310. doi:10.1103/PhysRevE. 89.042405.

[188] J. Duval, J. Lyklema, J. M. Kleijn, H. P. van Leeuwen, Amphifunctionally Electrified Interfaces: Coupling of Electronic and Ionic Surface-Charging Processes, Langmuir 17 (2001) 7573-7581. doi:10.1021/la010833i.

[189] W. Tang, P. Kovalsky, B. Cao, T. D. Waite, Investigation of fluoride removal from low-salinity groundwater by single-pass constant-voltage capacitive deionization, Water Research 99 (2016) 112-121. doi:10.1016/j.watres.2016.04.047.

[190] W. Stumm, J. J. Morgan, Aquatic Chemistry - Chemical Equilibria And Rates In Natural Waters, John Wiley and Sons Ltd, 1995.

[191] A. Pernia, J. G. Norniella, J. Martin-Ramos, J. Diaz, J. Martinez, Up-Down Converter for Energy Recovery in a CDI Desalination System, IEEE Transactions on Power Electronics 27 (2012) 3257-3265. doi:10.1109/TPEL.2011.2180926.

[192] P. M. Biesheuvel, M. van Soestbergen, Counterion volume effects in mixed electrical double layers, Journal of Colloid and Interface Science 316 (2007) 490-499. doi:10.1016/j.jcis.2007.08.006.

[193] A. Hemmatifar, D. I. Oyarzun, J. W. Palko, S. A. Hawks, M. Stadermann, J. G. Santiago, Equilibria model for $\mathrm{pH}$ variations and ion adsorption in capacitive deionization electrodes, Water Research 122 (2017) 387-397. doi:10.1016/j. watres.2017.05.036.

[194] P. M. Biesheuvel, M. A. Cohen Stuart, Cylindrical cell model for the electrostatic free energy of polyelectrolyte complexes, Langmuir 20 (2004) 4764-4770. doi: 10.1021/1a0496789.

[195] S. Porada, A. Shrivastava, P. Bukowska, P. M. Biesheuvel, K. C. Smith, Nickel Hexacyanoferrate Electrodes for Continuous Cation Intercalation Desalination of Brackish Water, Electrochimica Acta 255 (2017) 369-378. doi:10.1016/j. electacta.2017.09.137.

[196] R. Ghalloussi, W. Garcia-Vasquez, N. Chaabane, L. Dammak, C. Larchet, S. V. Deabate, E. Nevakshenova, V. Nikonenko, D. Grande, Ageing of ion-exchange membranes in electrodialysis: A structural and physicochemical investigation, Journal of Membrane Science 436 (2013) 68-78. doi:10.1016/j.memsci.2013. 02.011. 


\section{List of publications}


- J.E. Dykstra, P.M. Biesheuvel, H. Bruning, A. ter Heijne, Theory of ion transport with fast acid-base equilibrations in bioelectrochemical systems, Physical Review E 90 (2014) 013302.

- T. Kim, J.E. Dykstra, S. Porada, A. van der Wal, J. Yoon, P.M. Biesheuvel, Enhanced charge efficiency and reduced energy use in capacitive deionization by increasing the discharge voltage, Journal of Colloid and Interface Science 446 (2015) 317-326.

- J.M. Paz-Garcia, J.E. Dykstra, P.M. Biesheuvel, H.V.M. Hamelers, Energy from $\mathrm{CO}_{2}$ using capacitive electrodes - A model for energy extraction cycles, Journal of Colloid and Interface Science 442 (2015) 103-109.

- J.E. Dykstra, R. Zhao, P.M. Biesheuvel, A. van der Wal, Resistance identification and rational process design in Capacitive Deionization, Water Research 88 (2016) 358-370.

- J.E. Dykstra, J. Dijkstra, A. van der Wal, H.V.M. Hamelers, S. Porada, On-line method to study dynamics of ion adsorption from mixtures of salts in capacitive deionization, Desalination 390 (2016) 47-52.

- G.J. Doornbusch, J.E. Dykstra, P.M. Biesheuvel, M.E. Suss, Fluidized bed electrodes with high carbon loading for water desalination by capacitive deionization, Journal of Materials Chemistry A 4 (2016) 3642-3647.

- G.J. Doornbusch, J.E. Dykstra, H.V.M. Hamelers, P.M. Biesheuvel, Method for Fluidized Bed Capacitive Deionization of a Fluid and De-ionization Device Therefor, Dutch Patent No. NL2015572B1 (2017).

- J.E. Dykstra, K.J. Keesman, P.M. Biesheuvel, A. van der Wal, Theory of $\mathrm{pH}$ changes in water desalination by capacitive deionization, Water Research 119 (2017) 178-186.

- T.M. Mubita, S. Porada, P.M. Biesheuvel, A. van der Wal, J.E. Dykstra, Capacitive deionization with wire-shaped electrodes, Electrochimica Acta 270 (2018) 165-173.

- J.E. Dykstra, S. Porada, A. van der Wal, P.M. Biesheuvel, Energy consumption in Capacitive Deionization - constant current versus constant voltage operation (submitted to Water Research).

- J.E. Dykstra, M.K. Struijs, G.C. Franken, S. Porada, A. van der Wal, Effect of dissolved oxygen on porous carbon electrodes in (Membrane) Capacitive Deionization (in preparation). 


\section{Acknowledgements}


Het promotieonderzoek is afgerond! Hoewel het natuurlijk een fijn gevoel is om het proefschrift voltooid te hebben, realiseer ik me vooral hoe mooi de afgelopen jaren zijn geweest. Het was fantastisch om vier jaar lang de vrijheid te hebben om te onderzoeken en te leren. Er zijn veel mensen die hebben bijgedragen aan de totstandkoming van dit proefschrift, aan het leerproces of aan de ontspanning en gezelligheid die voor een fijne afwisseling hebben gezorgd, waar ik erg dankbaar voor ben.

Bert, ik ben je zeer dankbaar voor de mogelijkheid die je aan mij hebt geboden om een promotieonderzoek te doen. Wij hebben vele waardevolle gesprekken gehad. Jouw enthousiasme voor (M)CDI en je drive voor gedegen en in alle rust uitgevoerd onderzoek om inzicht te verwerven in de technologie, heb ik erg gewaardeerd. Je was niet alleen geïnteresseerd in de resultaten en conclusies van het onderzoek, maar je wilde ook altijd precies weten hoe de theorie was opgezet en hoe de experimenten waren uitgevoerd. Bedankt voor je kritische houding!

Maarten, bedankt voor jouw enthousiasme, betrokkenheid en vertrouwen! Ik bewonder je enorme precisie bij het bestuderen van theorie en bij het lezen van manuscripten. Bovendien lukt het je altijd om extreem snel te reageren en neem je alle tijd en rust om zaken te bespreken. Daarnaast wil ik je bedanken voor alle interessante discussies die we hebben gehad over wetenschappelijke en maatschappelijke onderwerpen! Onze gesprekken over maatschappelijke interacties en verantwoordelijkheden zijn altijd erg levendig en resulteren vaak in nieuwe inzichten. Dankjewel daarvoor!

I would like to thank the members of the Thesis committee. Frans Leermakers, Matthew Suss, Rob Lammertink and Luuk Rietveld, thank you for the time you took to critically read my Thesis. Matthew, special thanks to you for travelling from Haifa to Wageningen for the defence.

Het onderzoek is helemaal uitgevoerd bij Wetsus in Leeuwarden. Ik wil alle mensen bedanken die ervoor zorgen dat Wetsus een fantastisch onderzoeksinstituut is met zeer goede voorzieningen en een erg leuke werksfeer! Wetsus is een unieke plek waar iedereen bijdraagt aan het fijne Wetsus-gevoel. Ik wil een aantal collega's in het bijzonder noemen.

Cees, Johannes en Bert, ik ben jullie dankbaar voor alle kansen en mogelijkheden die Wetsus biedt, zowel voor het doen van onderzoek, als voor persoonlijke ontwikkeling. Hoewel Wetsus blijft groeien, zorgen jullie ervoor dat de dynamische sfeer en het persoonlijke contact tussen collega's niet gehinderd worden door ingewikkelde regels en procedures. Daarnaast wil ik Ernst, Jan, Harm, Wim, Piet, Jan-Jurjen, Harry, John, Rienk, Gerrit, Wiebe, Janneke, Mieke, Marianne, Jan Willem, Ton en Jelmer bedanken voor de behulpzaamheid bij technische uitdagingen op het lab en op kantoor. Ook wil ik Linda, Anke, Helena, Roely, Jeanette, Trienke, Geke, Jannie, Tineke, Lucy en Jan bedanken voor alle administratieve 
hulp.

My office mates, Pau and Mariana ("old building") and later on also Sam, Hector, Prashanth, Fei, Rebeca and Gosha ("new building"), and thereafter Joeri, Jordi and Monir ("new building thesis writing room"), thank you for the great company, for many nice conversations and all the fun we had.

Also, I would like to thank a few CDI colleagues. Ran, when I started at Wetsus and you were about to leave, you taught me all the tricks to perform nice CDI experiments in the lab. Taeyoung, the first few months we worked together on a project and I learned a lot from you! Slawek, special thanks to you! It was great to discuss many (experimental) challenges on CDI with you, but it was even better to chat about many other things!

I would like to thank my students, Tania, Gijs, Marije and Geert for all their work, which has been published or will be submitted. But, most importantly, all of you, thank you for all your enthusiasm!

Verder wil ik de bedrijven in het thema Capacitive Deionization bedanken. Ik heb de themavergaderingen en interessante gesprekken met jullie over het onderzoek gewaardeerd.

In 2015 Wetsus organized the European Summer School on Electrochemical Engineering. We, Sam, Mariana, Pau, Tim, Jorrit, Víctor, Jan Willem, Jordi and I, formed the local organizing committee for this week. We were a great team and we had a lot of fun organizing this week. Thank you guys!

De hardlooptochten met collega's waren voor mij een belangrijk onderdeel van het Wetsus-gevoel: Janneke, Jorrit, Bianca en Sanne, bedankt voor onze (bijna ;)) tweewekelijkse lunchtochtjes. Samen hebben we in 2015 onze eerste halve marathon gelopen, wat was dat een ervaring! Prashanth, also many thanks to you, when I started my PhD project I could not even imagine I would be able to run a marathon, but thanks to a crazy idea, we did it! Diego, also thanks for our morning runs.

Furthermore, I would like to thank Rik, Paula, Lucía, Heleen, Michele, Karel, Tom, Louis, Jaap, Doekle, Hester, Riet, Gerben, Roel, Casper, Marianne, Tania and Hilde. To all of you thank you for the interesting conversations and nice chats we had!

Hoewel het onderzoek is uitgevoerd bij Wetsus, ben ik verbonden geweest aan de vakgroep Milieutechnologie in Wageningen. Het was altijd erg leuk om langs te komen in Wageningen. Daarnaast, Huub, ben ik jou dankbaar voor het vertrouwen dat je hebt gegeven om mij, na afronding van mijn promotieonderzoek, bij Milieutechnologie aan de slag te laten gaan. Verder wil ik mijn collega's Harry, Annemiek, Kasia, Momo, Nora, Alette, David, Tim, Miriam, Hardy, Jan, Marjo, Liesbeth, Marjolein, Ludo, Arnoud, Ilse, Koen, Joeri, Jess, Laura en andere collega's bij Milieutechnologie bedanken voor de warme ontvangst en leuke 
gesprekken.

$\mathrm{Nu}$ is het tijd om alle andere vrienden en familie te bedanken. De vele gezellige en interessante gesprekken en leuke uitstapjes hebben voor een fijne afwisseling de afgelopen jaren gezorgd. Zonder deze afwisseling en gezelligheid was het onmogelijk geweest om dit proefschrift te schrijven.

Wieneke en Nico, jullie rol in de totstandkoming van dit proefschrift is niet te onderschatten! Toen wij het vak Water Treatment samen volgden en wij, een beetje toevallig, samen in een groep terecht kwamen, hadden wij erg veel lol bij het ontwerpen van een waterzuiveringsinstallatie. Onze regelmatig terugkerende afspraken, nu ook met Bas en Emmy, waarbij de vrolijkheid al begint bij de receptie van het museum waar we hebben afgesproken, zijn altijd geslaagd!

Karmijn, Evelien, Marlies, Jan, Nico, Emmy, Andy, Laura, Ewoud, Rhodé, Niels, Frazen en Bastiaan, na onze WSO-tijd blijven we elkaar zo'n twee keer per jaar zien. Onze regelmatig terugkerende niet-traditionele kerstdinertjes en weekendjes weg zijn altijd erg gezellig en geslaagd!

Lars, Steven, Nelleke, Mariëlle, Sander, Paul, Pauline en Nolan, bedankt voor de vele zeilweekenden, zomerzeilweken en weekendjes weg, bedankt voor de vriendschap!

Kawire, Imke, Anna, Wietse, Robin, Leoni, Jeroen en Yanniek, bedankt voor alle gezelligheid de afgelopen jaren! Bedankt voor de vele gezellige zeilweekenden, fietsweekenden, vakanties, memorabele kanotochtjes, wandelingen en gesprekken! Jeroen, hartstikke bedankt voor het ontwerpen van de omslag van mijn proefschrift!

Verder wil ik een aantal andere vrienden bedanken. Marion, wat leuk dat we een jaar geleden samen begonnen zijn met een theoriecursus wadzeilen en dat we daarna, mede dankzij jouw enthousiasme, samen een klein bootje hebben gekocht! De introductie en discussie van dit boekje zijn op ons bootje geschreven. Roos, bedankt voor alle leuke gesprekken, wandelingen en etentjes! Kasper, bedankt voor onze vele gesprekken over heel veel verschillende maatschappelijke onderwerpen! Froukje, tankewol foar ús itentsjes de ôfrûne jierren! Jan-Martijn, tankewol foar ús freonskip en goeie gesprekken, al sûnt de middelbere skoalle! Mariana, special thanks to you, thanks for all the fun and happiness during our Wetsus time!

$\mathrm{Nu}$ wil ik mijn paranimfen bedanken. Marijke, het was geweldig om samen tweeëneenhalf jaar in Leeuwarden te hebben gewoond aan de Zuidergrachtswal! We hebben deze mooie tijd meermaals gevierd met onze geweldige huisfeesten, waarbij het soms de vraag was of we de volgende dag nog een vloer zouden hebben. In deze tijd heb je veel meegekregen van het promotieleven en ik wil je bedanken voor alle gezelligheid en goede gesprekken. Gelukkig was het afsluiten van ons huis in Leeuwarden zeker niet het einde van onze vriendschap en hebben 
we elkaar de afgelopen tijd nog veel gesproken en gezien. Leoni, mijn AID-zusje, bedankt voor de vriendschap, al sinds onze Algemene Introductiedagen in Wageningen! Het is gaaf dat we al zoveel dingen samen hebben gedaan: een studie, een bestuursjaar, meerdere vakanties, weekendjes weg, (lange en intensieve ;)) fietstochten, heel veel etentjes en eindeloze (telefoon)gesprekken. Jouw steun en praktische hulp tijdens de afronding van dit promotieonderzoek waren erg fijn! Leoni en Marijke, ik ben erg blij dat jullie mijn paranimfen zijn, bedankt! dutch

Leave famylje, tankewol foar alle gesellichheid en waarmte de ôfrûne jierren! Beppe, Epy \& Albert, Gerry \& Roelof, Albert \& Myra, Luit \& Jamie, Elske, Ittie, tankewol foar alles! Jimme wiene der altiten, ek wannear't it net maklik wie. Tankewol foar de goeie steun en ynteresse!

Leave heit en Jannie, tankewol foar alles. Alle gesprekken oan 'e tafel, earst yn Waskemar, en letter yn Mitslawier, hawwe enoarm hulpen! Us konversaasjes, oer sosjale saken, oer it libben en oer deistiche dingen, binne altiten goed. Heit, tankewol foar alle waarmte, fertrouwen, soargen en stimulâns! 

Jouke Eabele Dykstra (1989) was born in Drachten, The Netherlands. He grew up in Waskemeer, a nearby village in southeast Fryslân. After finishing high school in 2007, he enrolled with Wageningen University to study Environmental Sciences, to obtain his MSc degree in 2013. During his studies, he was a full-time board member of the Student Union WSO. A minor was conducted on the topic of Modeling and Identification of Dynamic Systems. His MSc

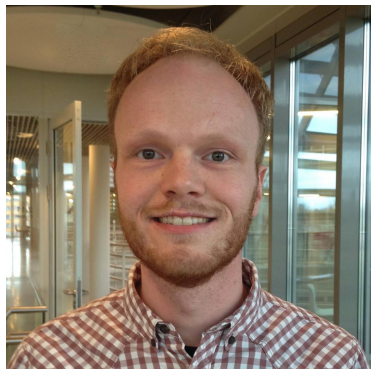
Thesis entitled "Modeling electrokinetics and electrochemistry in aqueous electrolytes" aimed to improve the understanding of ion transport and electrochemical reactions in bioelectrochemical systems for water treatment, and was published in 2014 in Physical Review E. During his Internship at the Institute of New Materials in Saarbrücken, Germany, he constructed theoretical models to describe ion adsorption in Capacitive Deionization, a water desalination technology. After he graduated with honors in 2013, he started as a PhD student with Wageningen University seconded to Wetsus, European Centre of Excellence for Sustainable Water Technology, located in Leeuwarden, The Netherlands. Here, he developed a theoretical computer model to improve the understanding of resistances and energy losses, ion-selective adsorption and unwanted faradaic reactions in Capacitive Deionization. Furthermore, he contributed to the organization of an international scientific conference in 2014 and a summer school on electrochemical engineering in 2015. Also, he enjoyed to contribute to the course Chemical Reactor Design in the joint-degree program Water Technology (Wetsus Academy), and he supervised four MSc students. Currently, he works at the Sub-department of Environmental Technology at Wageningen University, where he focusses on the development of technology for (selective) water desalination relevant to allocate water resources between different users. 


\section{SENSE}

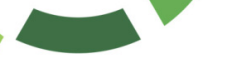

Netherlands Research School for the

Socio-Economic and Natural Sciences of the Environment

\section{I P L O M A}

For specialised PhD training

The Netherlands Research School for the

Socio-Economic and Natural Sciences of the Environment

(SENSE) declares that

\section{Jouke Eabele Dykstra}

born on 23 June 1989 in Smallingerland, The Netherlands

has successfully fulfilled all requirements of the

Educational Programme of SENSE.

Wageningen, 15 June 2018

the Chairman of the SENSE board

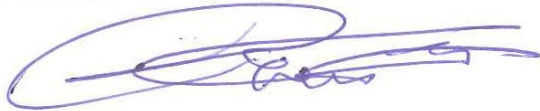

Prof. dr. Huub Rijnaarts the SENSE Director of Education

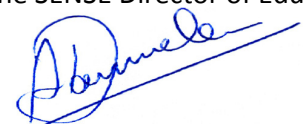

Dr. Ad van Dommelen

The SENSE Research School has been accredited by the Royal Netherlands Academy of Arts and Sciences (KNAW)

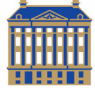

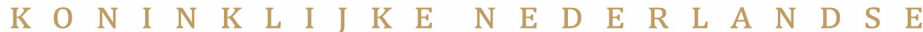

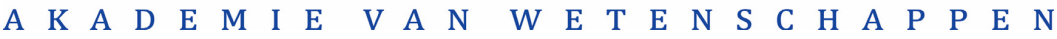




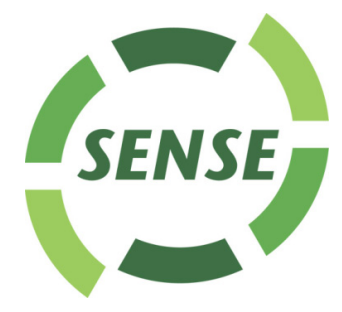

The SENSE Research School declares that Jouke Eabele Dykstra has successfully fulfilled all requirements of the Educational PhD Programme of SENSE with a work load of $40.8 \mathrm{EC}$, including the following activities:

\section{SENSE PhD Courses}

- Environmental research in context (2014)

- Research in context activity: 'Co-organizing and chairing organizing committee of 7th European Summer School on Electrochemical Engineering (Leeuwarden, Netherlands 22-26 June 2015)'

\section{Selection of other PhD and Advanced MSc Courses}

- Adobe InDesign Essential Training, Wageningen University (2013)

- Supervising MSc. students, Wetsus, Leeuwarden (2014)

- European Summer School on Electrochemical Engineering, European Federation of Chemical Engineers, Leeuwarden (2015)

- Image Processing for Scientists: Adobe Illustrator \& InDesign, Wetsus, Leeuwarden (2016)

- Techniques for Writing and Presenting a Scientific Paper, Wageningen University (2016)

\section{Management and Didactic Skills Training}

- Supervising four MSc students with thesis (2014-2017)

○ Teaching in the MSc course 'Chemical Reactor Design' (2013-2017)

\section{Oral Presentations}

- Enhanced Energy Efficiency in Increased Discharging Voltage Capacitive Deionization. Interfaces in Water and Environmental Science, 26-28 May 2014, Leeuwarden, The Netherlands

- Combined Electrical Double Layer and Ion Transport Modeling in Water Desalination. International Congress on Industrial and Applied Mathematics, 10-14 August 2015, Beijing, China

- Modeling of Acid-base Equilibria in Capacitive Deionization. International Conference on Capacitive Deionization and Electrosorption, 26-29 October 2015, Saarbrücken, Germany

- Theory on Faradaic reactions, Acid-base Equilibria, Ion transport and Adsorption in (Membrane) Capacitive Deionization. International Conference on Capacitive Deionization, Electrosorption \& Electrodialysis, 3-6 July 2017, Seoul, Republic of Korea

SENSE Coordinator PhD Education

Dr. Peter Vermeulen 
This work was performed in the cooperation framework of Wetsus, European Centre of Excellence for Sustainable Water Technology (www.wetsus.eu). Wetsus is co-funded by the Dutch Ministry of Economic Affairs and Climate Policy and Ministry of Infrastructure and Water Management, the Province of Fryslân, and the Northern Netherlands Provinces. The author likes to thank the participants of the research theme "Capacitive Deionization" for the fruitful discussions and their financial support. 



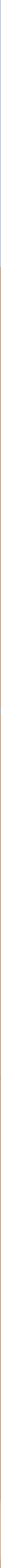

